\title{
A C*-ALGEBRAIC FRAMEWORK FOR QUANTUM GROUPS
}

\author{
T. MASUDA, Y. NAKAGAMI, AND S. L. WORONOWICZ
}

\begin{abstract}
We develop a general framework to deal with the unitary representations of quantum groups using the language of $C^{*}$-algebras. Using this framework, we prove that the duality holds in a general context. This extends the framework of the duality theorem using the language of von Neumann algebras previously developed by Masuda and Nakagami.
\end{abstract}

\section{INTRODUCTION}

When we look at quantum groups, we still have the shortage of examples which are studied in detail from all points of view. This applies mostly to noncompact groups. As long as we deal with the examples of the quantum groups of the compact type, the existence of the Haar measure, the (relative) invariance of the Haar measure with respect to the scaling automorphism group etc. are all clear even from a very general point of view. Due to the Peter-Weyl theorem for quantum groups of classical type, we can characterize the object by making use of the set of all matrix elements $W=\left\{w_{i j}\right\}_{i j}$ of all possible finite dimensional (unitary) representations without using functional analysis. However, even in the compact case, the $\mathrm{C}^{*}$-algebraic framework is essential to guarantee the existence of the Haar measure, from which almost all the theory of representations has been developed 33 .

However, once we become interested in quantum groups of non-compact type, we are faced for instance with a serious necessity for the convergence of the infinite sums of matrix elements of infinite dimensional unitary representations. Furthermore, the Haar measures correspond to unbounded linear functionals and the elements which generate the coordinate ring of the quantum group are unbounded linear operators, which cause analytical difficulties. Therefore we are obliged to work with some appropriate notions and the basic tools of functional analysis, in particular the language of operator algebras. Despite these difficulties, there are some quite explicitly studied quantum groups like quantum Lorentz groups, $E_{q}(2)$, quantum ' $a z+b$ ' group, quantum ' $a x+b$ ' group, $\widetilde{S U}_{q}(1,1)$ etc. of non-compact type [22, 38, 32, 36, 37, 11], 10] with infinite dimensional irreducible unitary representations. Furthermore we are still discovering new explicitly computable quantum groups of non-compact type. All of them should be investigated from the point of view of algebra, analysis and geometry.

Now, the history of the operator algebraic study of the classical locally compact groups (with the duality theorem) goes back to Stinespring who transferred the regular representation to what we nowadays call the multiplicative unitary operator for the purpose of generalizing the TannakaKrein's duality to unimodular locally compact groups. Then it was Kac and Takesaki who independently used this unitary operator to extend the duality theorem for the category which is much wider than that of locally compact groups. Immediately after that, it was Tatsuuma who recognized the importance of this unitary operator and named it the "Kac-Takesaki operator". After that, Enock-Schwartz and Kac-Vainermann completed the theory of generalized locally compact groups, which they called "Kac algebras" and introduced the "fundamental unitary" playing the role of Kac-Takesaki operator. The details concerning the Kac algebras including the history of the development of the subject (before the appearance of quantum groups) can be found in their book 7 . It is also remarked that in the development of those theoretical frameworks to deal with the general theory of locally compact groups including the duality theorem, all the achievements were made on the basis of von Neumann algebras by the heavy use of the Tomita-Takesaki theory. The only approach based on the language of $\mathrm{C}^{*}$-algebras was proposed by Vallin [26], in which the theory was not developed in full generality due to the lack of the reasonable integration theory for $\mathrm{C}^{*}$-algebras. It is also emphasized that the condition which we now call the strong (right) invariance had been used from the early stage for the development of the subject.

In the middle eighties, after the famous discoveries of Leningrad school, Drinfeld and Jimbo introduced quantum groups as deformations of enveloping algebras of semisimple Lie algebras. At 
the beginning these objects were of purely algebraic nature. In particular only finite-dimensional representations and no $*$-involutions were considered.

A little later the third author introduced a noncommutative analogue of the algebras of functions on compact groups. Then the $*$-involution was an essential part of the considered structure. It allowed to distinguish compact and noncompact quantum groups and to consider unitary representations of quantum groups. For noncompact groups unitary representations can be infinite dimensional which requires the use of analytical methods. One of the aims of this publication is to draw attention to the importance of the functional analytical methods from this point of view.

It was the paper of Baaj and Skandalis [1] who first gave a reasonable framework to deal with such problems on the basis of the multiplicative unitary operator. Their approach avoided the discussion of Haar measures while the duality and the fact that the dual of the dual is isomorphic to the original object were a trivial consequence of the definitions. Their theory in large part worked with $\mathrm{C}^{*}$-algebras.

In 19, the first and the second authors gave an alternative formulation of the theory including the discussion of the Haar measures with the duality being a deep theorem. That paper was based on the straightforward use of the Tomita-Takesaki theory to deal with the Haar measure following the above mentioned theory of Kac algebras. We were obliged to sacrifice the topological nature of the underlying quantum groups which we believe to be important nowadays. This is why we need a reasonable framework to deal with the quantum groups as a generalization of locally compact groups. For this purpose, the right choice of the language and the axioms are important.

Here we would like to list a number of requirements that any good axiomatization of the theory of locally compact quantum groups should satisfy. In our opinion the quantum theory should be developed in the way parallel to the classical one. In particular this rule determines the choice of basic concepts. Classically the groups are locally compact spaces endowed with a group rule. By the famous Gelfand-Naimark theorem locally compact spaces are in one to one correspondence with commutative $\mathrm{C}^{*}$-algebras (unital if the considered spaces are compact). Following the general philosophy, passage to quantum spaces consists in using noncommutative algebras. Consequently the underlying spaces of quantum groups should be described by noncommutative (in general) $\mathrm{C}^{*}$-algebras. The use of von Neumann algebras seems not to be appropriate. They correspond (in commutative case) to measure spaces. Of course, one is able to develop the theory of groups with underlying spaces being measure spaces, but this theory is less natural and more complicated than the theory of locally compact groups.

The next reason why one should work with the language of $\mathrm{C}^{*}$-algebras is the more natural description of homomorphisms of (quantum) groups. Assume that we have a locally compact subgroup $H$ (of lower dimension) of a locally compact group $G$. Within the $\mathrm{C}^{*}$-algebra language, the inclusion $H \subset G$ is described by the restriction map $\left.C_{0}(G) \ni a \rightarrow a\right|_{H} \in C_{\text {bounded }}(H)$, which is a morphism (in the sense of 31] cf. Definition A.4 from $C_{0}(G)$ into $C_{0}(H)$. In the von Neumann algebra setting the corresponding restriction map $L^{\infty}(G) \rightarrow L^{\infty}(H)$ does not make sense.

Now we pass to the second ingredient of the concept of a group which is the binary operation called the group rule. Let $G$ be a group. Classically the group rule is a continuous mapping from $G \times G$ onto $G$. Passing to the quantum theory we replace cartesian product of spaces by tensor product of algebras (alternative choice would be the free product of algebras but then all the classical groups would be lost). Let $\alpha$ be a $\mathrm{C}^{*}$-norm on the algebraic tensor product $A \otimes_{\text {alg }} B$ of $\mathrm{C}^{*}$-algebras $A$ and $B$. By definition, tensor product $A \otimes_{\alpha} B$ is defined as the completion of $A \otimes$ alg $B$ with respect to the norm $\alpha$.

Let $A$ be a $\mathrm{C}^{*}$-algebra related to a quantum group. The correspondence between spaces and algebras is a contravariant functor. It changes the direction of morphisms. Therefore the group rule corresponds to a morphism $\delta$ from $A$ into $A \otimes_{\alpha} A$. This is the so called comultiplication (we use small letter $\delta$, because the symbol $\Delta$ is reserved to denote the modular operator in TomitaTakesaki theory). In this context one has to pay some attention to the notion of morphism of $\mathrm{C}^{*}$-algebras. The usual *-algebra homomorphisms are not appropriate to use in the context of quantum spaces. They correspond (in the commutative case) to the proper continuous mappings of underlying locally compact spaces. On the other hand the group rule is not a proper mapping from $G \times G$ into $G$ if $G$ is not compact. Therefore one has to modify the notion of morphism creating a new category of $\mathrm{C}^{*}$-algebras. Restricted to commutative algebras the category should be (anti)-equivalent (via Gelfand Naimark theorem) to the category of locally compact spaces and 
(all) continuous mappings. Such a category of $\mathrm{C}^{*}$-algebras was proposed in [29] (see also [26], [30], [31]). For reader's convenience we recalled the definition of morphisms of $\mathrm{C}^{*}$-algebras in Appendix A(Definition A.4).

In general we have more than one $\mathrm{C}^{*}$-norm on $A \otimes_{\mathrm{alg}} A$. Among them there is the minimal one that corresponds to the spatial tensor product of $\mathrm{C}^{*}$-algebras denoted simply by $A \otimes A$. Due to the minimality, for any $\mathrm{C}^{*}$-norm $\alpha$ we have canonical epimorphism $\Psi_{\alpha}: A \otimes_{\alpha} A \rightarrow A \otimes A$. Replacing $\delta$ by $\Psi_{\alpha}{ }^{\circ} \delta$ we obtain a morphism from $A$ into $A \otimes A$. Therefore the choice of spatial tensor product as the operation corresponding to the cartesian product of spaces produces the theory with larger (than with other choices) class of examples. It is also worth mentioning that in most known examples the algebra $A$ is nuclear and all tensor products $A \otimes_{\alpha} A$ coincide.

Summing up: The basic concepts of the theory of locally compact quantum groups should be a $\mathrm{C}^{*}$-algebra (say $A$ ) together with a morphism $\delta \in \operatorname{Mor}(A, A \otimes A$ ). In the last expression ' $\otimes$ ' and 'Mor' denote the spatial tensor product of $\mathrm{C}^{*}$-algebras and the set of morphisms in the sense of Definition A.4

If $A$ is commutative then by Gelfand Naimark theory $A=C_{\infty}(G)$, where $G$ is a locally compact topological space and $\delta(a)\left(g, g^{\prime}\right)=a\left(g g^{\prime}\right)$, where

$$
G \times G \ni\left(g, g^{\prime}\right) \longmapsto g g^{\prime} \in G
$$

is a continuous mapping.

Now we shall discuss the most desired system of axioms. Quantum groups with commutative $A$ should coincide with the usual groups. In this case our axioms should reduce to the axioms or simple theorems of the theory of locally compact topological groups. This requirement is satisfied by axioms of the coassociativity of $\delta$, the properness and the cancellation law (see Definitions 1.2 and 1.3 in the next section). The coassociativity clearly corresponds to the associativity of the group rule (0.1): $\left(g_{1} g_{2}\right) g_{3}=g_{1}\left(g_{2} g_{3}\right)$ for any $g_{1}, g_{2}, g_{3} \in G$. The properness means that the mappings $G \times G \ni\left(g, g^{\prime}\right) \mapsto\left(g, g g^{\prime}\right) \in G \times G$ and $G \times G \ni\left(g, g^{\prime}\right) \mapsto\left(g g^{\prime}, g^{\prime}\right) \in G \times G$ are proper i.e: $\left(g, g g^{\prime}\right)$ and $\left(g g^{\prime}, g^{\prime}\right)$ tend to infinity when $\left(g, g^{\prime}\right)$ tends to infinity. The cancellation property states that for any $g, g_{1}, g_{2} \in G$ we have:

$$
\begin{aligned}
& \left(g g_{1}=g g_{2}\right) \Rightarrow\left(g_{1}=g_{2}\right), \\
& \left(g_{1} g=g_{2} g\right) \Rightarrow\left(g_{1}=g_{2}\right) .
\end{aligned}
$$

A good candidate for an axiom is the statement of Theorem [5.16. Combined with the properness and cancellation law it reduces (for commutative $A$ ) to the postulate saying that the relations $g=h h^{\prime}$ and $h=g g^{\prime}$ define a homeomorphism

$$
G \times G \ni\left(g, g^{\prime}\right) \longmapsto\left(h, h^{\prime}\right) \in G \times G
$$

acting on $G \times G$. Unfortunately at present we are not able to base our theory on axioms of the form similar to the statement mentioned above.

By simple theorems of the theory of locally compact topological groups (the phrase used in the previous paragraph) we mean statements that may be derived from axioms by short and easy reasoning. In principle they should be found (explicitly or implicitly) on first pages of handbooks devoted to the subject. The Haar theorem stating the existence of the right (or left) invariant measure does not belong to the category of simple theorems. In a good theory of locally compact quantum groups, the existence of the Haar weight should be one of advanced theorems.

This is why the existing axiomatizations of the theory of locally compact groups are not satisfactory. In all the approaches (including that of Kustermans and Vaes [15] and the one used in the present paper) the existence of the Haar measure is included as one of the axiom of the theory. We strongly believe that this is a temporary situation and that in the future we shall have a theory that will satisfy the requirements listed above. To find a satisfactory axiomatization it is important to understand well the logical relations between the basic concepts and statements of the theory. This is the main purpose of the present paper.

Taking the above discussions into account, we aim to present a reasonable framework, as general as possible, to formulate (within the formalism of $\mathrm{C}^{*}$-algebras) the objects which we recognize as locally compact quantum groups. We are going to improve our previous approach [19] based on the language of von Neumann algebras. Our first results were announced by the third author at Fields Institute and by the first author at Banach Center in 1995. Then the first author also announced our 
preliminary results in an expository article [17. On the way, the third author modified the theory of multiplicative unitaries [34] replacing the (semi)-regularity condition of Baaj and Skandalis by a new one better suited to our aims. While the present version of our paper was being developed, Kustermans and Vaes [15] found an alternative approach to the locally compact quantum groups starting from a completely different set of axioms and proving the duality theorem. However, in both cases, the existence of the Haar measure(s) is an inevitable assumption. This means that the both are still on the way to be completed. The existence of the Haar measure should be derived from a more primitive set of axioms.

Now we have a final comment on the strong right invariance which is one of our axioms. It is the only axiom that relates the antipode with remaining structure (comultiplication, Haar measure). On the other hand, Kustermans and Vaes [15] proposed to assume the existence of both the right and the left Haar measures. Then the existence of the antipode is a sophisticated theorem. It seems that, in both cases, those conditions are essentially related to the manageability which plays an important role in both approaches.

In [19] we discussed the properties of the special one parameter automorphism groups which we now call the scaling groups. This is the symmetry first recognized in [18 which describes an obstruction of our object to being a locally compact group or a Kac algebra. We also remark that there are many improvements of the proofs as well as the basic axioms comparing with what is given in 19:

(1) The main definition 1.5 is simplified: the commutativity of the coproduct with the unitary antipode as well as with the scaling group; and the commutativity of the left and the right Haar weights; are not assumed.

(2) In case of weights (contrary to the states) the commutativity of the two weights $\varphi$ and $\psi$ does not imply that the Connes' Radon-Nikodym cocycle $(D \psi: D \varphi)_{t}$ is invariant under the group of modular automorphism $\left\{\sigma_{t}^{\varphi}\right\}_{t \in \mathbb{R}}$.

(3) There is a gap in the proof of Lemma 2.14 in [19. The assertion is true by Theorem 6.10 of the present paper.

(4) The invariance of the Haar weight with respect to the scaling automorphisms is replaced by relative invariance.

The relative invariance (instead of the invariance) of the Haar weight under the scaling group appeared first in the paper of Kustermans and Vaes [15] as a result of their axioms. Recently, Van Daele [27. found that the quantum groups ' $a x+b$ ' and ' $a z+b$ ' introduced in 36, 37] have Haar measures which are relatively invariant but not invariant with respect to the scaling automorphisms. To include such examples in our framework we assume the relative invariance of the Haar weight. This modification was very easy, it resulted in a number of minor changes.

Now we summarize the organization of this paper. In Section [1] we give our basic definitions and the axioms of weighted Hopf $\mathrm{C}^{*}$-algebra which we discuss throughout this paper. Then using the GNS construction we introduce the Hilbert space and reveal the content of the strong right invariance. Section 2]is devoted to an investigation of matrix elements of the Kac-Takesaki operator. We prove the theorems describing the coalgebraic properties of scaling group and unitary antipode. In Section 3 we show that the Kac-Takesaki operator is a multiplicative unitary and that it is manageable in the sense of [34]. Then the general theory of multiplicative unitaries enable us to introduce the basic objects related to the dual weighted Hopf $\mathrm{C}^{*}$-algebra. In Section 4 we discuss the Radon-Nikodym derivative of the left Haar weight with respect to the right one. This derivative is determined by two operators $\rho$ and $\gamma$. Later in Section [ 6 we show that $\rho$ is affiliated with the considered $\mathrm{C}^{*}$-algebra and $\gamma$ is a multiple of unity related to the constant describing the relative invariance of the Haar weight. In the last part of Section 4 we prove the uniqueness of the antipode and the Haar weight. Section 5 is devoted to the properties of an operator $\widehat{\Delta}$ which plays an important role in our analysis. In Section 6 we introduce the dual GNS-mapping $\widehat{\eta}$. Operator $\widehat{\Delta}$ introduced in the previous section turns out to be the modular operator related to $\widehat{\eta}$. At the end of this section we discuss the behaviour of the (right invariant) Haar weight with respect to left shifts. In Section 7 we introduce the dual Haar weight. This way the construction of dual weighted Hopf $\mathrm{C}^{*}$-algebra is achieved and the duality is shown. In our work, we deal all the time with the "regular" representations and the "regular" dual in order to have faithful dual Haar weight. This means that our multiplicative unitary operator is the "regular" bicharacter. However, we are also able to discuss the universal dual with the universal bicharacter. This topic will be discussed in a separate publication. Section $[$ is devoted to the quantum codouble construction which is a dual 
version to the Drinfeld's quantum double. It helps to deal with examples like quantum Lorentz groups.

Then we have six Appendices ranging from $\mathrm{A}$ to $\mathbb{E}$ This is where we collect the technicalities used in our paper. A few fundamental facts on the multiplier algebra and the definition of the set $\operatorname{Mor}(A, B)$ of morphisms are given in Appendix $\mathrm{A}$ Appendix $\mathrm{B}$ contains the concepts of GNS mapping, its commutant (and the corresponding bicommutant theorem) and (exact) vector presentation of a GNS mapping. These concepts provide a convenient language for the TomitaTakeski theory outlined in Appendix $\mathbb{D}$ Appendix $[\mathbf{C}$ is devoted to weights and their properties (such as strict faithfulness) on a separable $\mathrm{C}^{*}$-algebras. Tensor products of the GNS-mappings are discussed in Appendix $\mathbb{E}$ In the last Appendix $\mathbf{E}$ we present the basic properties of analytic generators of one parameter groups acting on Banach spaces.

Some remarks on the terminology used in this paper: First of all, we decided to use the name "weighted Hopf $\mathrm{C}^{*}$-algebra" for the basic object of this paper. We have to acknowledge that the terminology "Hopf $\mathrm{C}^{*}$-algebra" is already used in the paper of Vallin [26] and also in the paper of Baaj and Skandalis 1 in a different context. The word "weighted" means that we deal with the object with a Haar weight. Then, the one parameter family of automorphism which comes from the "radial part" of the antipode is called the scaling group. (In 19, we called it the deformation automorphism group.) We hope that our choice of the terminology will not cause any confusion to the readers.

Another change which we have to acknowledge is the choice of the particular form of KacTakesaki operator $W$. In general, the Kac-Takesaki operator is introduced by choosing a certain linear mapping acting on the tensor product of the function algebra by itself and pushing it down to the Hilbert space level. There are several possibilities. In [19] we used the mapping

$$
a \otimes b \longmapsto \delta(b)(a \otimes 1) .
$$

This choice forced us to work with the left invariant Haar weight and with the strong left invariance axiom. In the present paper, we replace (0.2) by the mapping

$$
a \otimes b \longmapsto \delta(a)(1 \otimes b) .
$$

Consequently we work with the right invariant Haar weight and the strong right invariance. This choice is consistent with the notational convention of Baaj and Skandalis [1] and the framework developed in 34 .

Acknowledgement. The first two authors are grateful to the Department of Mathematical Methods in Physics of the University of Warsaw, and the last two authors are grateful to the Department of Applied Mathematics of Fukuoka University and the Department of Mathematics of Norwegian University of Science and Technology. The first author was partially supported by the Grant-in-Aid for Scientific Research (No. 11440052) and the second author was partially supported by the Grant-in-Aid for Scientific Research (No. 12640216), the Ministry of Education, Science, Sports and Culture, Japan. The third author was supported by Polish KBN, grants No 2 P301 020 07, No 2 P0A3 03014 and No 2 P03A 040 22, by Foundation for Polish Science and by Japan Society for Promoting Science.

\section{BASIC DEFINITIONS AND AXIOMS}

The main aim of this section is to introduce the notion of a weighted Hopf $C^{*}$-algebra.

Some remarks on used notation. The unit $1=1_{A}$ of a $C^{*}$-algebra $A$ always means the unit element of the multiplier algebra $M(A)$. Then id denotes the identity mapping. For the inner product the physicist convention is used, i.e. $(y \mid x)$ is linear in $x$ and conjugate linear in $y$. Throughout this paper, all $C^{*}$-algebras and all Hilbert spaces are assumed to be separable. Moreover all tensor products of $C^{*}$-algebras are minimal tensor products unless specially mentioned. We shall use compositions of the form $\varphi^{\circ} \kappa$, where $\varphi \in A^{*}$ and $\kappa$ is a closed operator acting on $A$. We write $\varphi \circ \kappa \in A^{*}$ if there exists a $\psi \in A^{*}$ such that $\psi(a)=\varphi(\kappa(a))$ for all $a \in \mathcal{D}(\kappa)$. Clearly $\psi$ is unique and is denoted by $\varphi \circ \kappa$.

To understand well the forthcoming definitions and results the reader is advised to look at Appendices $\mathrm{A}$ and $[\mathrm{C}$ where the basic notions such as multipliers, morphisms and weights are explained.

Now, we are ready to give our basic definition of the weighted Hopf $C^{*}$-algebra. 
Definition 1.1. A pair $(A, \delta)$ where $A$ is a $C^{*}$-algebra and $\delta \in \operatorname{Mor}(A, A \otimes A)$ satisfying the coassociativity condition $\left(\delta \otimes \operatorname{id}_{A}\right)^{\circ} \delta=\left(\mathrm{id}_{A} \otimes \delta\right)^{\circ} \delta$ is called a $C^{*}$-bialgebra.

The mapping $\delta$ will be called a comultiplication or a coproduct. In what follows we denote by $1=1_{A}$ the unit of the multiplier algebra $M(A)$. The relation $\delta \in \operatorname{Mor}(A, A \otimes A)$ does not mean that $\delta(a) \in A \otimes A$ for $a \in A$. We know only that $\delta(a) \in M(A \otimes A)$. It means that $\delta(a)(b \otimes c) \in A \otimes A$ and $(b \otimes c) \delta(a) \in A \otimes A$ for any $a, b, c \in A$. Therefore the following definitions are relevant:

Definition 1.2. $A C^{*}$-bialgebra $(A, \delta)$ is said to be proper if $\delta(a)(1 \otimes b)$ and $(b \otimes 1) \delta(a)$ belong to $A \otimes A$ for any $a, b, c \in A$.

Definition 1.3. Let $(A, \delta)$ be a proper $C^{*}$-bialgebra. We say that $(A, \delta)$ has the cancellation property if the linear spans of the sets $\{\delta(a)(1 \otimes b): a, b \in A\}$ and $\{(b \otimes 1) \delta(a): a, b \in A\}$ are dense in $A \otimes A$.

Let $(A, \delta)$ be a proper $\mathrm{C}^{*}$-bialgebra. Then for any continuous linear functionals $\varphi, \psi \in A^{*}$ and any $a \in A$, the convolution products

$$
\begin{aligned}
& \varphi * a:=(\mathrm{id} \otimes \varphi) \delta(a), \\
& a * \psi:=(\psi \otimes \mathrm{id}) \delta(a) .
\end{aligned}
$$

belong to $A$. Indeed, by Proposition A.2 we may assume that $\varphi=b \varphi^{\prime}$ and $\psi=\psi^{\prime} c$, where $\varphi^{\prime}, \psi^{\prime} \in A^{*}$ and $b, c \in A$. Then

$$
\begin{aligned}
& \varphi * a=\left(\mathrm{id} \otimes \varphi^{\prime}\right)\{\delta(a)(1 \otimes b)\}, \\
& a * \psi=\left(\psi^{\prime} \otimes \mathrm{id}\right)\{(c \otimes 1) \delta(a)\}
\end{aligned}
$$

and our statement follows immediately from the properness of the considered bialgebra. We shall also use the convolution product of functionals:

$$
\varphi * \psi:=(\varphi \otimes \psi) \circ \delta \in A^{*} .
$$

One can easily verify that

$$
(\varphi * \psi)(a)=\varphi(\psi * a)=\psi(a * \varphi) .
$$

Due to the coassociativity of the comultiplication, the convolution product obey the following associativity laws:

$$
\begin{aligned}
& (\psi * \varphi) * a=\psi *(\varphi * a), \\
& (a * \psi) * \varphi=a *(\psi * \varphi), \\
& (\varphi * a) * \psi=\varphi *(a * \psi), \\
& (\varphi * \chi) * \psi=\varphi *(\chi * \psi)
\end{aligned}
$$

In these formulae $a \in A$ and $\varphi, \chi, \psi \in A^{*}$.

Throughout the paper, the concept of a right invariant weight on a $\mathrm{C}^{*}$-bialgebra plays an essential role. In brief, $h$ is right invariant if $(h \otimes \mathrm{id}) \delta(a)=h(a) 1$.

Definition 1.4. Let $(A, \delta)$ be a $C^{*}$-bialgebra and $h$ be a locally finite, lower semicontinuous weight on $A$. We say that $h$ is right invariant if for any $a \in A_{+}$such that $h(a)<\infty$ and any $\varphi \in A_{+}^{*}$ we have

$$
h(\varphi * a)=\varphi(1) h(a) .
$$

We shall use standard notation: For any weight $h$ we set:

$$
\begin{aligned}
\mathfrak{N}_{h} & =\left\{a \in A: h\left(a^{*} a\right)<\infty\right\}, \\
\mathfrak{M}_{h} & =\left\{a \in A_{+}: h(a)<\infty\right\}^{\text {linear span }} \\
& =\left\{a^{*} b: a, b \in \mathfrak{N}_{h}\right\}^{\text {linear span }}
\end{aligned}
$$

It is known that $h$ extends uniquely to a linear functional on $\mathfrak{M}_{h}$.

Assume that $h$ is right invariant. For any $\varphi \in A_{+}^{*}$ the mapping $a \mapsto \varphi * a=\left(\operatorname{id}_{A} \otimes \varphi\right)(\delta(a))$ is completely positive. Hence, by the Kadison inequality

$$
\{\varphi * a\}^{*}\{\varphi * a\} \leq \varphi(1)\left\{\varphi *\left(a^{*} a\right)\right\} .
$$

Now, using the right invariance we see that

$$
h\left((\varphi * a)^{*}(\varphi * a)\right) \leq \varphi(1)^{2} h\left(a^{*} a\right)<\infty .
$$


for any $a \in \mathfrak{N}_{h}$. Remembering that any $\varphi \in A^{*}$ can be written by a linear combination of four positive functionals we conclude that $\varphi * a \in \mathfrak{N}_{h}$ for any $a \in \mathfrak{N}_{h}$ and $\varphi \in A^{*}$. Therefore for any $a, b \in \mathfrak{N}_{h}$ and any $\varphi, \psi \in A^{*}$ the products $\left(\varphi^{*} * a\right)^{*} b=\left(\varphi * a^{*}\right) b$ and $a^{*}(\psi * b)$ belong to $\mathfrak{M}_{h}$ and expressions $h\left(\left(\varphi * a^{*}\right) b\right)$ and $h\left(a^{*}(\psi * b)\right)$ make sense. We shall use them in our main definition.

The object which we study in this paper is defined as follows.

Definition 1.5. Let $(A, \delta)$ with $A$ separable be a proper $C^{*}$-bialgebra with the cancellation property. $W e$ say that $(A, \delta)$ is a weighted Hopf $C^{*}$-algebra if there exist a closed densely defined linear map $\kappa$ acting on $A$ (called antipode) and a locally finite strictly faithful lower semicontinuous right invariant weight $h$ on $A$ (called Haar weight) such that

(1) The operator $\kappa$ admits the following polar decomposition:

$$
\kappa=R o \tau_{i / 2}
$$

where $\tau_{i / 2}$ is the analytic generator of a one parameter group $\tau=\left\{\tau_{t}\right\}_{t \in \mathbb{R}}$ of automorphisms of the $C^{*}$-algebra $A$ (called scaling group) and $R$ is an involutive antiautomorphism of $A$ (called unitary antipode) commuting with automorphisms $\tau_{t}$ for all $t \in \mathbb{R}$.

(2) The weight $h$ is relatively invariant under $\left\{\tau_{t}\right\}_{t \in \mathbb{R}}$ : there exists a positive scalar $\lambda$ such that $h{ }^{\circ} \tau_{t}=\lambda^{t} h$ for all $t \in \mathbb{R}$.

(3) (Strong right invariance) For any $\varphi \in A^{*}$ with $\varphi^{\circ} \kappa \in A^{*}$ we have

$$
h\left(\left(\varphi * a^{*}\right) b\right)=h\left(a^{*}\left(\left(\varphi^{\circ} \kappa\right) * b\right)\right)
$$

for all $a, b \in \mathfrak{N}_{h}$.

It should be noted that the scaling constant $\lambda$ is 1 for the most examples of quantum groups constructed so far. The first examples with $\lambda \neq 1$ appeared in 36, 37] (cf. 27]). This possibility was foreseen for the first time in [15].

It is understood that the group $\tau$ is pointwise norm continuous: for any $a \in A,\left\|\tau_{t}(a)-a\right\| \rightarrow 0$ when $t \rightarrow 0$. We refer to Appendix $\mathbb{E}$ for the definition of the analytic generator. Clearly, $\kappa^{2}=\tau_{i}$. Performing the holomorphic continuation in the formula $\tau_{t}(a b)=\tau_{t}(a) \tau_{t}(b)$ we see that $\mathcal{D}\left(\tau_{i / 2}\right)$ is a subalgebra in $A$ and $\tau_{i / 2}(a b)=\tau_{i / 2}(a) \tau_{i / 2}(b)$ for any $a, b \in \mathcal{D}\left(\tau_{i / 2}\right)$. Similarly performing the holomorphic continuation in the formula $\tau_{t}\left(a^{*}\right)=\tau_{t}(a)^{*}$ we see that $\mathcal{D}\left(\tau_{i / 2}\right)^{*}=\mathcal{D}\left(\tau_{-i / 2}\right)$ and $\tau_{-i / 2}\left(a^{*}\right)=\tau_{i / 2}(a)^{*}$ for all $a \in \mathcal{D}\left(\tau_{i / 2}\right)$. Therefore $\left(\tau_{i / 2} \mathcal{D}\left(\tau_{i / 2}\right)\right)^{*}=\mathcal{D}\left(\tau_{i / 2}\right)$ and $\tau_{i / 2}\left(\tau_{i / 2}(a)^{*}\right)^{*}=a$ for any $a \in \mathcal{D}\left(\tau_{i / 2}\right)$. Remembering that $R$ is an involutive antiautomorphism of $A$ commuting with $\tau_{t}$ we conclude that

$$
\begin{gathered}
\kappa(a b)=\kappa(b) \kappa(a), \\
\kappa\left(\kappa(a)^{*}\right)^{*}=a
\end{gathered}
$$

for any $a, b \in \mathcal{D}(\kappa)$. In other words the mapping $a \mapsto \kappa(a)^{*}$ is a conjugate linear multiplicative involution acting on $\mathcal{D}(\kappa)$.

Remark 1.6. The polar decomposition (1.3) is unique. The scaling group and the unitary antipode are determined by $\kappa$. Indeed $\tau_{i}=\kappa^{2}$ and using Corollary [F.4 we obtain the uniqueness of $\tau$. In particular $\tau_{i / 2}$ is unique and the uniqueness of $R$ follows immediately from (1.3).

Remark 1.7. We have to point out that the automorphism $\tau_{t}$ was denoted in [19 by $\tau_{t / 2}$ and called the deformation automorphism. However, in this paper, we call it the scaling automorphism following the terminology of 34 .

Remark 1.8. The strong right invariance is a condition which establishes the connection between comultiplication and antipode: it replaces the well known formula

$$
\mathrm{m}(\mathrm{id} \otimes \kappa) \delta(a)=\mathrm{m}(\kappa \otimes \mathrm{id}) \delta(a)=e(a) 1
$$

of the usual theory of Hopf algebras. This means the following. If $A$ is a Hopf algebra, then the linear mapping $W: A \otimes A \rightarrow A \otimes A$ defined by $a \otimes c \mapsto \delta(a)(1 \otimes c)$ (where $a, c \in A$ ) is bijective due to the existence of the explicit inverse $V: b \otimes d \mapsto\left(\operatorname{id}_{A} \otimes \kappa\right)(\delta(b))(1 \otimes d)$. The proof that $V=W^{-1}$ uses (1.4). On the Hilbert space level $W$ should unitary. Therefore we require the equality $W^{*}=V$, i.e.

$$
(h \otimes h)\left(\left(1 \otimes c^{*}\right) \delta\left(a^{*}\right)(b \otimes d)\right)=(h \otimes h)\left(\left(a^{*} \otimes c^{*}\right)(\mathrm{id} \otimes \kappa)(\delta(b))(1 \otimes d)\right) .
$$

This is the strong right invariance with $\varphi(x)=h\left(c^{*} x d\right)$.

Some of our main assertions in this paper are summarized as follows: 
Theorem 1.9. Let $(A, \delta)$ be a weighted Hopf $C^{*}$-algebra. Then

(1) The unitary antipode $R$, the scaling group $\left\{\tau_{t}\right\}_{t \in \mathbb{R}}$ and the Haar weight $h$ are uniquely determined.

(2) The coproduct $\delta$ commute with the unitary antipode and the scaling group:

$$
\delta \circ R=\sigma o(R \otimes R) \circ \delta, \quad \delta \circ \tau_{t}=\left(\tau_{t} \otimes \tau_{t}\right) \circ \delta, \quad t \in \mathbb{R},
$$

where $\sigma$ is the flip automorphism on $A \otimes A$.

(3) There exists a pointwise norm continuous one parameter $*$-automorphism group $\left\{\sigma_{t}\right\}_{t \in \mathbb{R}}$ of $A$ such that

$$
h\left(\sigma_{i / 2}(a) \sigma_{i / 2}(a)^{*}\right)=h\left(a^{*} a\right)
$$

for any $a \in \mathcal{D}\left(\sigma_{i / 2}\right)$. The group $\left\{\sigma_{t}\right\}_{t \in \mathbb{R}}$ is the modular automorphism related to the Haar weight $h$. Clearly, the above formula is the KMS-condition for $h$.

(4) For any $t \in \mathbb{R}$ we have

$$
\delta \sigma_{t}=\left(\sigma_{t} \otimes \tau_{t}\right) \circ \delta .
$$

(5) There exists a strictly positive element $\rho$ affiliated with the $C^{*}$-algebra $A$ such that for any $a \in A_{+}$with $h(a)<\infty$ and any $\varphi \in A_{+}^{*}$ we have

$$
h(a * \varphi)=\varphi(\rho) h(a),
$$

where by definition $\varphi(\rho)=\lim _{n \rightarrow \infty} \varphi\left(\frac{n \rho}{n 1+\rho}\right)$. Moreover we have $: \delta(\rho)=\rho \otimes \rho, \tau_{t}(\rho)=\rho$ and $R(\rho)=\rho^{-1}$.

(6) The composition $h^{L}=h^{\circ} R$ is the left Haar weight on A. The Connes' Radon-Nikodym cocycle is of the form

$$
\left(D h^{L}: D h\right)_{t}=\lambda^{-i t^{2} / 2} \rho^{i t},
$$

where $\lambda$ is the constant appearing in Definition 1.5, Moreover $\sigma_{t}(\rho)=\lambda^{-t} \rho$.

The strong right invariance plays an essential role in our theory. We end this Section with a number of equivalent formulations of this condition. By the way we introduce Hilbert space objects that will be used throughout of the paper. The most important one is the Kac-Takesaki operator $W$ (in other words the right regular representation of the considered quantum group). In this (and the next) section the matrix elements of $W$ are defined and their properties are investigated. The operator itself will be introduced in Section 3

Till the end of this section we shall deal with a proper $\mathrm{C}^{*}$-bialgebra $(A, \delta)$ with the cancellation property equipped with a faithful locally finite, lower semicontinuous right-invariant weight $h$ and with a closed densely defined linear map $\kappa$ satisfying the first two conditions of Definition [1.5] Therefore our results will be independent of the strong right invariance condition and the strict faithfulness of the weight $h$.

Using the GNS construction we obtain a Hilbert space $\mathcal{H}$ and a representation $\pi$ of $A$ acting on $\mathcal{H}$. Since $h$ is faithful, so is $\pi$ and we shall identify $A$ with its $\pi$-image: $A \subset \mathcal{L}(\mathcal{H})$. The corresponding GNS map will be denoted by $\eta$. It is a densely defined closed linear mapping from $A$ into $\mathcal{H}$ with the domain $\mathcal{D}(\eta)=\mathfrak{N}_{h}=\left\{a \in A: h\left(a^{*} a\right)<\infty\right\}$ such that

$$
(\eta(c) \mid a \eta(b))=h\left(c^{*} a b\right), \quad a \in A, \quad b, c \in \mathcal{D}(\eta),
$$

The GNS mapping $\eta$ plays a crucial role in the entire paper. It is closed with respect to the strong topology on $A$ and the norm topology in $\mathcal{H}$. The separability of $A$ implies that of the Hilbert space $\mathcal{H}$. See Appendices $\mathbb{B}$ and $\mathbb{C}$ for the details.

We start with some technicalities which we use throughout this paper. For any $x, y \in \mathcal{H}, \omega_{x, y}$ will denote the functional on $\mathcal{L}(\mathcal{H})$ such that

$$
\omega_{x, y}(a)=(x \mid a y)
$$

for $a \in \mathcal{L}(\mathcal{H})$. It is clear that the set of all such functionals is linearly dense in the predual $\mathcal{L}(\mathcal{H})_{*}$ of $\mathcal{L}(\mathcal{H})$. In general, if $A$ is a $C^{*}$-algebra acting on $\mathcal{H}$ then $A$ is endow with the $\sigma$-weak topology coming from $\mathcal{L}(\mathcal{H})$. In what follows $A_{*}$ will denote the set of all $\sigma$-weakly continuous functionals on $A$. It is known that

$$
A_{*}=\left\{\left.\varphi\right|_{A}: \varphi \in \mathcal{L}(\mathcal{H})_{*}\right\} .
$$

Consequently we set $A_{*+}=A_{*} \cap A_{+}$.

We already know (see the paragraph containing formula (1.2)) that

$$
\varphi * a \in \mathcal{D}(\eta)
$$


for any $a \in \mathcal{D}(\eta)$ and any $\varphi \in A^{*}$.

Proposition 1.10. For any $\varphi \in A^{*}$, there exists $W_{\varphi} \in \mathcal{L}(\mathcal{H})$ such that

$$
W_{\varphi} \eta(a)=\eta(\varphi * a)
$$

for any $a \in \mathcal{D}(\eta)$. The mapping $A^{*} \ni \varphi \mapsto W_{\varphi} \in \mathcal{L}(\mathcal{H})$ is injective, linear and bounded. Moreover,

$$
W_{\varphi} W_{\psi}=W_{\varphi * \psi}
$$

for all $\varphi, \psi \in A^{*}$.

Proof. Formula (1.6) defines $W_{\varphi}$ on a dense linear subset of $\mathcal{H}$. We have to show that $W_{\varphi}$ is bounded. If $\varphi$ is positive then using (1.2) we get $\|\eta(\varphi * a)\| \leq \varphi(1)\|\eta(a)\|$ and $\left\|W_{\varphi}\right\| \leq \varphi(1)$. For general $\varphi$ we have $\left\|W_{\varphi}\right\| \leq 4\|\varphi\|$. This is because any continuous linear functional on $A$ is a linear combination of four positive functionals with norms not larger than the norm of the original functional.

If $W_{\varphi}=0$ then $\varphi * a=0$ for all $a \in A$. Hence $(\operatorname{id} \otimes \varphi)((b \otimes 1) \delta(a))=b(\varphi * a)=0$ for all $a, b \in A$ and using the cancellation property we get $\varphi=0$. It shows that the mapping $\varphi \mapsto W_{\varphi}$ is injective.

Formula (1.7) follows immediately from the associativity of the convolution product.

Proposition 1.11. For every $x, y \in \mathcal{H}$, there exists a unique element $W(x, y) \in A$ such that

$$
\varphi(W(x, y))=\left(x \mid W_{\varphi} y\right)
$$

for any $\varphi \in A^{*}$. Furthermore, $W(x, y)$ is sesquilinear in $(x, y)$ and $\|W(x, y)\| \leq 4\|x\|\|y\|$.

Proof. The uniqueness, the sesquilinearity and the estimate follow from the formula. It is enough to prove the existence of operators $W(x, y)$ for a dense set of vectors $x, y$. We shall use the commutant $\eta^{\prime}$ of the GNS map $\eta$ (see Appendix (B). For any $a \in \mathcal{D}(\eta), a^{\prime} \in \mathcal{D}\left(\eta^{\prime}\right)$ and $x \in \mathcal{H}$ we put :

$$
W\left(a^{\prime *} x, \eta(a)\right):=a * \omega_{x, \eta^{\prime}\left(a^{\prime}\right)} .
$$

Then for any $\varphi \in A^{*}$ we have

$$
\begin{aligned}
\varphi\left(W\left(a^{\prime *} x, \eta(a)\right)\right) & =\varphi\left(a * \omega_{x, \eta^{\prime}\left(a^{\prime}\right)}\right)=\left(x \mid(\varphi * a) \eta^{\prime}\left(a^{\prime}\right)\right) \\
& =\left(x \mid a^{\prime} \eta(\varphi * a)\right)=\left(a^{\prime *} x \mid W_{\varphi} \eta(a)\right),
\end{aligned}
$$

where the second equality due to the cyclic property of the convolution product. This proves the assertion.

Proposition 1.12. The set $\{W(x, y): x, y \in \mathcal{H}\}$ is linearly dense in $A$.

Proof. Suppose $\varphi \in A^{*}$ satisfies $\varphi(W(x, y))=0$ for all $x, y \in \mathcal{H}$. Then $(x \mid \eta(\varphi * a))=$ $\left(x \mid W_{\varphi} \eta(a)\right)=\varphi(W(x, \eta(a)))=0$ and $\varphi * a=0$ for all $a \in \mathcal{D}(\eta)$. Therefore $W_{\varphi}=0$ and hence $\varphi=0$. This proves the assertion.

Theorem 1.13. Let $(A, \delta)$ be a proper $C^{*}$-bialgebra with the cancellation property, $h$ be a faithful locally finite, lower semicontinuous right invariant weight on $A$ and $\kappa$ be a closed densely defined linear map acting on A. Assume that the first two conditions of Definition 1.5 are satisfied. Then the following statements are equivalent:

1. The strong right invariance (Condition 3 of Definition 1.5).

2. For all $\varphi \in A^{*}$ such that $\varphi^{\circ} \kappa \in A^{*}$ we have

$$
W_{\varphi^{*}}=\left(W_{\varphi^{o} \kappa}\right)^{*} .
$$

3. Formula (1.9) holds for all $\varphi$ from a weakly* dense subset $\mathcal{D}_{0}$ of $A^{*}$ such that $\psi^{\circ} \tau_{t} \in \mathcal{D}_{0}$ for all $\psi \in \mathcal{D}_{0}$ and $t \in \mathbb{R}$.

4. For any $x, y \in \mathcal{H}$, the element $W(x, y)$ is in the domain of $\kappa$ and $\kappa(W(x, y))=W(y, x)^{*}$. 
Proof. Assume that the strong right invariance holds. Then for any $\varphi \in A^{*}$ with $\varphi \circ \kappa \in A^{*}$ and any $a, b \in \mathcal{D}(\eta)$ we have

$$
\begin{aligned}
\left(W_{\varphi^{*}} \eta(a) \mid \eta(b)\right) & =\left(\eta\left(\varphi^{*} * a\right) \mid \eta(b)\right)=h\left(\left(\varphi * a^{*}\right) b\right) \\
& =h\left(a^{*}\left(\left(\varphi^{\circ} \kappa\right) * b\right)\right)=\left(\eta(a) \mid \eta\left(\left(\varphi^{\circ} \kappa\right) * b\right)\right)=\left(\eta(a) \mid W_{\varphi^{\circ} \kappa} \eta(b)\right),
\end{aligned}
$$

where we used the equality $\varphi * a^{*}=\left(\varphi^{*} * a\right)^{*}$ for the second equality. Remembering that the range of $\eta$ is dense in $\mathcal{H}$, we obtain relation (1.9). It shows that Statement 1 implies Statement 2.

Clearly Statement 2 implies Statement 3. Assume that Statement 3 holds. Then for any $x, y \in \mathcal{H}$ and any $\varphi \in \mathcal{D}_{0}$ we have

$$
\begin{aligned}
\left(\varphi^{\circ} \kappa\right)(W(x, y)) & =\left(x \mid W_{\varphi^{\circ} \kappa} y\right)=\left(W_{\varphi^{*}} x \mid y\right) \\
& =\overline{\left(y \mid W_{\varphi^{*}} x\right)}=\overline{\varphi^{*}(W(y, x))}=\varphi\left(W(y, x)^{*}\right) .
\end{aligned}
$$

Inserting $\varphi \circ \tau_{t}$ instead of $\varphi$, multiplying the both sides by $e^{-t^{2}}$, integrating over $t \in \mathbb{R}$ and remembering that $\kappa$ commutes with $\tau_{t}$, we obtain

$$
(\varphi \circ \kappa)\left(\mathcal{R}_{\tau}(W(x, y))=\varphi\left(\mathcal{R}_{\tau}\left(W(y, x)^{*}\right)\right),\right.
$$

where $\mathcal{R}_{\tau}$ is a linear operator acting on $A$ introduced by the formula

$$
\mathcal{R}_{\tau}(a)=\frac{1}{\sqrt{\pi}} \int_{-\infty}^{\infty} e^{-t^{2}} \tau_{t}(a) d t
$$

for any $a \in A$. It is known (cf. Appendix $\mathbb{E}$ ) that the range of $\mathcal{R}_{\tau}$ is contained in $\mathcal{D}\left(\tau_{i / 2}\right)=\mathcal{D}(\kappa)$. Therefore $(\varphi \circ \kappa)\left(\mathcal{R}_{\tau}(W(x, y))=\varphi\left(\kappa\left(\mathcal{R}_{\tau}(W(x, y))\right)\right.\right.$. Remembering that $\varphi$ runs over a weakly* dense subset $\mathcal{D}_{0} \subset A^{*}$ we obtain

$$
\kappa\left(\mathcal{R}_{\tau}(W(x, y))\right)=\mathcal{R}_{\tau}\left(W(y, x)^{*}\right) .
$$

Theorem F.7 shows now that $W(x, y) \in \mathcal{D}(\kappa)$ and that $\kappa(W(x, y))=W(y, x)^{*}$. This shows that Statement 3 implies Statement 4.

To complete the proof we have to show that Statement 4 implies the strong right invariance. Let $a, b \in \mathfrak{N}_{h}$. Inserting $x=\eta(a)$ and $y=\eta(b)$ in Statement 4 , we see that $W(\eta(a), \eta(b)) \in \mathcal{D}(\kappa)$ and $\kappa(W(\eta(a), \eta(b)))=W(\eta(b), \eta(a))^{*}$. Therefore for any $\varphi \in A^{*}$ with $\varphi \circ \kappa \in A^{*}$ we have

$$
\begin{aligned}
h\left(\left(\varphi * a^{*}\right) b\right) & =\left(\eta\left(\varphi^{*} * a\right) \mid \eta(b)\right)=\overline{\left(\eta(b) \mid W_{\varphi^{*}} \eta(a)\right)}=\overline{\varphi^{*}(W(\eta(b), \eta(a)))} \\
& =\varphi\left(W(\eta(b), \eta(a))^{*}\right)=\varphi(\kappa(W(\eta(a), \eta(b)))) \\
& =\left(\varphi^{\circ} \kappa\right)(W(\eta(a), \eta(b)))=\left(\eta(a) \mid W_{\varphi^{\circ} \kappa} \eta(b)\right) \\
& =\left(\eta(a) \mid \eta\left(\left(\varphi^{\circ} \kappa\right) * b\right)\right)=h\left(a^{*}\left(\left(\varphi^{\circ} \kappa\right) * b\right)\right) .
\end{aligned}
$$

\section{Matrix elements of KaC-TAKesaki operator}

In this Section we derive the simplest consequences of axioms listed in Definition 1.5 Let $(A, \delta)$ be a weighted Hopf $\mathrm{C}^{*}$-algebra. We shall use the notation and results of the previous Section. In particular all four Statements of Theorem 1.13 hold.

We assumed that the weight $h$ satisfies the relative invariance condition: $h \circ \tau_{t}=\lambda^{t} h$ for $t \in \mathbb{R}$. Therefore $\mathcal{D}(\eta)$ is $\left\{\tau_{t}\right\}_{t \in \mathbb{R}}$ invariant and there exists a positive self-adjoint operator $Q$ on $\mathcal{H}$ with $\operatorname{ker}(Q)=\{0\}$ such that

$$
Q^{2 i t} \eta(a):=\lambda^{-t / 2} \eta\left(\tau_{t}(a)\right), \quad a \in \mathcal{D}(\eta) .
$$

In [19] the operator $Q^{2}$ was denoted by $H$. For any $a \in A$ and $b \in \mathcal{D}(\eta)$ we have

$$
Q^{2 i t} a \eta(b)=Q^{2 i t} \eta(a b)=\lambda^{-t / 2} \eta\left(\tau_{t}(a b)\right)=\lambda^{-t / 2} \tau_{t}(a) \eta\left(\tau_{t}(b)\right)=\tau_{t}(a) Q^{2 i t} \eta(b) .
$$

Therefore

$$
\tau_{t}(a)=Q^{2 i t} a Q^{-2 i t}
$$

for all $t \in \mathbb{R}$ and $a \in A$.

By Lemma C.5 the strict faithfulness of $h$ implies that the mapping $\eta(a) \mapsto \eta\left(a^{*}\right)$ (where $a$ runs over $\left.\mathcal{D}(\eta) \cap \mathcal{D}(\eta)^{*}\right)$ is a closable conjugate linear involution. We denote by $S$ its closure. Let $F:=S^{*}$. Then there exist an involutive antiunitary $J$ and a strictly positive self-adjoint operator $\Delta$ such that $S=J \Delta^{1 / 2}$ and $F=J \Delta^{-1 / 2}$ by the fact that $S^{2}=1$ on $\mathcal{D}\left(\Delta^{1 / 2}\right)$. 
Taking into account (2.1) we see that the set $\left\{\eta(a): a \in \mathcal{D}(\eta) \cap \mathcal{D}(\eta)^{*}\right\}$ is a $\left\{Q^{i t}\right\}_{t \in \mathbb{R}}$ - invariant core of $S$. Moreover for any $x=\eta(a)$ in this set we have

$$
\begin{gathered}
Q^{2 i t} S x=Q^{2 i t} \eta\left(a^{*}\right)=\lambda^{-t / 2} \eta\left(\tau_{t}\left(a^{*}\right)\right), \\
S Q^{2 i t} x=\lambda^{-t / 2} S \eta\left(\tau_{t}(a)\right)=\lambda^{-t / 2} \eta\left(\tau_{t}(a)^{*}\right)
\end{gathered}
$$

and the right hand sides of the above equalities coincide. Therefore $Q^{2 i t} S Q^{-2 i t}=S$ for any $t \in \mathbb{R}$. By the uniqueness of the polar decomposition, we have

$$
\begin{aligned}
& Q^{2 i t} \Delta Q^{-2 i t}=\Delta, \\
& Q^{2 i t} J Q^{-2 i t}=J .
\end{aligned}
$$

The first equation means that $\Delta$ strongly commutes with $Q$. The second one is equivalent to the formula

$$
J Q J=Q^{-1} .
$$

The operators $Q$ and $\Delta$ play an essential role in all our considerations.

Lemma 2.1. Let $x \in \mathcal{D}\left(\Delta^{1 / 2}\right)$ and $y \in \mathcal{D}\left(\Delta^{-1 / 2}\right)$. Then $W\left(J \Delta^{-1 / 2} y, J \Delta^{1 / 2} x\right)=W(y, x)^{*}$.

Proof. Since $\eta\left(\mathcal{D}(\eta) \cap \mathcal{D}(\eta)^{*}\right)$ is a core for $\Delta^{1 / 2}$, it is sufficient to prove the statement for $x=\eta(a)$ where $a \in \mathcal{D}(\eta) \cap \mathcal{D}(\eta)^{*}$. For any $\varphi \in A^{*}$, we have

$$
\begin{aligned}
\varphi\left(W\left(J \Delta^{-1 / 2} y, J \Delta^{1 / 2} x\right)\right) & =\left(J \Delta^{-1 / 2} y \mid W_{\varphi} \eta\left(a^{*}\right)\right) \\
& =\left(J \Delta^{-1 / 2} y \mid \eta\left(\varphi * a^{*}\right)\right)=\left(J \Delta^{-1 / 2} y \mid J \Delta^{1 / 2} \eta\left(\varphi^{*} * a\right)\right) \\
& =\left(\eta\left(\varphi^{*} * a\right) \mid y\right)=\overline{\left(y \mid W_{\varphi^{*}} \eta(a)\right)} \\
& =\overline{\varphi^{*}(W(y, \eta(a)))}=\varphi\left(W(y, \eta(a))^{*}\right) .
\end{aligned}
$$

Combining Statement 4 of Theorem 1.13 with Lemma 2.1 we get

$$
\kappa(W(x, y))=W\left(J \Delta^{-1 / 2} y, J \Delta^{1 / 2} x\right)
$$

for all $x \in \mathcal{D}\left(\Delta^{1 / 2}\right)$ and $y \in \mathcal{D}\left(\Delta^{-1 / 2}\right)$.

Proposition 2.2. For any $x, y \in \mathcal{H}$, we have

(1) $\tau_{t}(W(x, y))=W\left(\Delta^{i t} x, \Delta^{i t} y\right)$ for $t \in \mathbb{R}$.

(2) $R(W(x, y))=W(J y, J x)$.

Remark 2.3. This proposition shows that the unitary antipode and the scaling group are uniquely determined by the Haar weight. So is $\kappa$. Unfortunately the proof of the uniqueness of the Haar weight requires the prior knowledge of the uniqueness of $\kappa$. See the proof of Theorem 4.10

Proof. Let $x \in \mathcal{D}(\Delta)$ and $y \in \mathcal{D}\left(\Delta^{-1}\right)$. Then using twice (2.5), we get

$$
\begin{aligned}
W\left(\Delta x, \Delta^{-1} y\right) & =W\left(J \Delta^{-1 / 2} J \Delta^{1 / 2} x, J \Delta^{1 / 2} J \Delta^{-1 / 2} y\right) \\
& =\kappa\left(W\left(J \Delta^{-1 / 2} y, J \Delta^{1 / 2} x\right)\right) \\
& =\kappa^{2}(W(x, y))=\tau_{i}(W(x, y))
\end{aligned}
$$

It shows that $W(x, y) \in \mathcal{D}\left(\tau_{i}\right)$ and

$$
\tau_{i}(W(x, y))=W\left(\Delta x, \Delta^{-1} y\right) .
$$

Using now Theorem F.3 we obtain the first statement. Moreover, for any $x \in \mathcal{D}\left(\Delta^{1 / 2}\right)$ and $y \in \mathcal{D}\left(\Delta^{-1 / 2}\right)$, the element $W(x, y) \in \mathcal{D}\left(\tau_{i / 2}\right)$ and

$$
\tau_{i / 2}(W(x, y))=W\left(\Delta^{1 / 2} x, \Delta^{-1 / 2} y\right) .
$$

Combining this with [2.5), we see that $R\left(W\left(\Delta^{1 / 2} x, \Delta^{-1 / 2} y\right)\right)=W\left(J \Delta^{-1 / 2} y, J \Delta^{1 / 2} x\right)$ for $x \in$ $\mathcal{D}\left(\Delta^{1 / 2}\right)$ and $y \in \mathcal{D}\left(\Delta^{-1 / 2}\right)$. Now the continuity of $W$ shows that the second statement holds in full generality.

By the above proposition the set $\{W(x, y): x, y \in \mathcal{H}\}$ is $R$ and $\left\{\tau_{t}\right\}_{t \in \mathbb{R}}$ invariant. By Proposition 1.12 this set is linearly dense in $A$. Using Theorem F.5 we obtain

Corollary 2.4. The linear span of the set $\{W(x, y): x, y \in \mathcal{H}\}$ is a core for $\kappa$. 
Proposition 2.5. For any $\varphi \in A^{*}$, we have

(1) $W_{\varphi^{\circ} R}=J\left(W_{\varphi}\right)^{*} J$

(2) $W_{\varphi o}^{o \tau_{t}}=\Delta^{-i t} W_{\varphi} \Delta^{i t}$.

Proof. Ad 1. For $x, y \in \mathcal{H}$ and $\varphi \in A^{*}$, we have

$$
\begin{aligned}
\left(x \mid W_{\varphi^{\circ} R} y\right) & =\varphi \circ R(W(x, y))=\varphi(W(J y, J x)) \\
& =\left(J y \mid W_{\varphi} J x\right)=\left(J W_{\varphi} J x \mid y\right),
\end{aligned}
$$

where we used Proposition 2.2 for the second equality. This proves the equality $J\left(W_{\varphi}\right)^{*} J=W_{\varphi \circ R}$.

Ad 2. Similarly, we have

$$
\begin{aligned}
\left(x \mid W_{\varphi^{\circ} \tau_{t}} y\right) & =\varphi \circ \tau_{t}(W(x, y)) \\
& =\varphi\left(W\left(\Delta^{i t} x, \Delta^{i t} y\right)\right)=\left(\Delta^{i t} x \mid W_{\varphi} \Delta^{i t} y\right) .
\end{aligned}
$$

This proves the equality $\Delta^{-i t} W_{\varphi} \Delta^{i t}=W_{\varphi^{\circ} \tau_{t}}$.

Proposition 2.6. $\quad(1) \delta \circ R=\sigma o(R \otimes R) \circ \delta$, where $\sigma$ is the flip automorphism on $A \otimes A$.

(2) $\delta \circ \tau_{t}=\left(\tau_{t} \otimes \tau_{t}\right) \circ \delta$.

Proof. Ad 1. By Proposition 2.5 for any $\varphi, \psi \in A^{*}$ we have

$$
\begin{aligned}
W_{(\varphi * \psi)^{\circ} R} & =J\left(W_{\varphi * \psi}\right)^{*} J=J\left(W_{\varphi} W_{\psi}\right)^{*} J \\
& =J\left(W_{\psi}\right)^{*} J J\left(W_{\varphi}\right)^{*} J=W_{\psi \mathrm{o} R} W_{\varphi \mathrm{o} R} .
\end{aligned}
$$

By Proposition 1.10, $W_{\varphi}=0$ implies $\varphi=0$. Therefore $(\varphi * \psi) \circ R=(\psi \circ R) *(\varphi \circ R)$ and hence we obtain $(\varphi \otimes \psi) \circ \delta \circ R=(\varphi \otimes \psi) \circ \sigma \circ(R \otimes R) \circ \delta$ for $\varphi, \psi \in A^{*}$. This proves Assertion 1 .

Ad 2. By Proposition 2.5] we have

$$
\begin{aligned}
W_{\left(\varphi \circ \tau_{t}\right) *\left(\psi^{\circ} \tau_{t}\right)} & =W_{\varphi^{\circ} \tau_{t}} W_{\psi^{\circ} \tau_{t}}=\left(\Delta^{-i t} W_{\varphi} \Delta^{i t}\right)\left(\Delta^{-i t} W_{\psi} \Delta^{i t}\right) \\
& =\Delta^{-i t} W_{\varphi} W_{\psi} \Delta^{i t}=\Delta^{-i t} W_{\varphi * \psi} \Delta^{i t}=W_{(\varphi * \psi) \circ} \tau_{t} .
\end{aligned}
$$

We then have $\left(\varphi \circ \tau_{t}\right) *\left(\psi \circ \tau_{t}\right)=(\varphi * \psi) \circ \tau_{t}$ and hence we obtain

$$
\begin{aligned}
(\varphi \otimes \psi)\left(\left(\tau_{t} \otimes \tau_{t}\right) \circ \delta\right) & =\left(\left(\varphi \circ \tau_{t}\right) \otimes\left(\psi \circ \tau_{t}\right)\right) \circ \delta \\
& =\left(\varphi \circ \tau_{t}\right) *\left(\psi \circ \tau_{t}\right)=(\varphi * \psi) \circ \tau_{t}=(\varphi \otimes \psi) \circ \delta \circ \tau_{t}
\end{aligned}
$$

for all $\varphi, \psi \in A^{*}$. This proves Assertion 2 .

Corollary 2.7. $\delta{ }^{\circ} \kappa=\sigma^{o}(\kappa \otimes \kappa) \circ \delta$ on $\mathcal{D}(\kappa)$.

Proof. By Assertion 2 of Proposition [2.6] $a \in \mathcal{D}\left(\tau_{i / 2}\right)$ if and only if $\delta(a) \in \mathcal{D}\left(\tau_{i / 2} \otimes \tau_{i / 2}\right)$. Since $\mathcal{D}(\kappa)=\mathcal{D}\left(\tau_{i / 2}\right)$ and $\mathcal{D}(\kappa \otimes \kappa)=\mathcal{D}\left(\tau_{i / 2} \otimes \tau_{i / 2}\right)$, our statement is immediate from Proposition 2.6

Lemma 2.8. (1) $Q^{2 i t} W_{\varphi} Q^{-2 i t}=W_{\varphi^{o} \tau_{-t}}$ for $\varphi \in A^{*}$ and $t \in \mathbb{R}$.

(2) For any $x, y \in \mathcal{H}$, we have $\tau_{t}(W(x, y))=W\left(Q^{2 i t} x, Q^{2 i t} y\right)$ for $t \in \mathbb{R}$.

Proof. Ad 1. Let $\varphi \in A^{*}$ and $a \in \mathcal{D}(\eta)$. Then

$$
\begin{aligned}
\tau_{-t}\left(\varphi * \tau_{t}(a)\right) & =\tau_{-t^{\circ}}\left(\mathrm{id}_{A} \otimes \varphi\right) \circ \delta\left(\tau_{t}(a)\right) \\
& =\left(\operatorname{id}_{A} \otimes \varphi \circ \tau_{t}\right) \circ \delta(a)=\left(\varphi \circ \tau_{t}\right) * a
\end{aligned}
$$

and applying $\eta$ to the both sides, we get

$$
Q^{-2 i t} W_{\varphi} Q^{2 i t} \eta(a)=W_{\varphi^{\circ} \tau_{t}} \eta(a) .
$$

Ad 2. For $x, y \in \mathcal{H}$ and $\varphi \in A^{*}$, we have

$$
\begin{aligned}
\varphi\left(\tau_{t}(W(x, y))\right) & =\left(x \mid W_{\varphi^{\circ} \tau_{t}} y\right) \\
& =\left(x \mid Q^{-2 i t} W_{\varphi} Q^{2 i t} y\right)=\varphi\left(W\left(Q^{2 i t} x, Q^{2 i t} y\right)\right)
\end{aligned}
$$

by Assertion 1. This completes the proof.

Proposition 2.9. Let $\varphi, \psi \in A^{*}$. Assume that $W_{\varphi}^{*}=W_{\psi}$. Then $\psi=\varphi^{*}$ o . 
Proof. We have to show that $\psi(a)=\varphi^{*}(\kappa(a))$ for any $a \in \mathcal{D}(\kappa)$. By Corollary 2.4 we may assume that $a=W(x, y)$, where $x, y \in \mathcal{H}$. Now we compute

$$
\begin{aligned}
\psi(W(x, y)) & =\left(x \mid W_{\psi} y\right)=\left(W_{\varphi} x \mid y\right)=\overline{\left(y \mid W_{\varphi} x\right)}=\overline{\varphi(W(y, x))} \\
& =\varphi^{*}\left(W(y, x)^{*}\right)=\varphi^{*}(\kappa(W(x, y))) .
\end{aligned}
$$

\section{Pentagonal equation and manageability}

In this section, we construct the Kac-Takesaki operator $W$ and prove that this operator is a multiplicative unitary. We also prove that $W$ is manageable in the sense of [34].

We start with a lemma of Dini type.

Lemma 3.1. Let $\left\{a_{n}\right\}_{n \in \mathbb{N}}$ be an increasing sequence of elements in $A_{+}$.

(1) If $a \in A$ and for any $\psi \in A_{+}^{*}$, the sequence $\left\{\psi\left(a_{n}\right)\right\}_{n \in \mathbb{N}}$ converges to $\psi(a)$, then the sequence $\left\{a_{n}\right\}_{n \in \mathbb{N}}$ converges in norm to $a$.

(2) If for any $\omega \in A_{+}^{*}$, the sequence $\left\{\omega\left(a_{n}\right)\right\}_{n \in \mathbb{N}}$ converges to $\|\omega\|$, then the sequence $\left\{a_{n}\right\}_{n \in \mathbb{N}}$ converges strictly to the unit 1 of $M(A)$.

Proof. Ad 1. We put $\Lambda:=\left\{\psi \in A_{+}^{*}:\|\psi\| \leq 1\right\}$. Then $\Lambda$ is a compact subset of $A^{*}$ with respect to the weak ${ }^{*}$ topology. By the assumption, $\left\{\psi(a)-\psi\left(a_{n}\right)\right\}_{n \in \mathbb{N}}$ is a decreasing sequence converging to zero. By the Dini's Theorem this convergence is uniform on $\Lambda$. Therefore we have

$$
\left\|a-a_{n}\right\|=\sup _{\psi \in \Lambda}\left|\psi(a)-\psi\left(a_{n}\right)\right| \rightarrow 0 .
$$

as $n$ goes to infinity and the assertion follows.

Ad 2. Since $\omega\left(a_{n}\right) \leq\|\omega\|$ for all $\omega \in A_{+}^{*}, a_{n}$ satisfies $0 \leq a_{n} \leq 1$. Let $b \in A$. Then the sequence $\left\{b^{*} a_{n} b\right\}_{n \in \mathbb{N}}$ is increasing and for any $\psi \in A_{+}^{*}, \psi\left(b^{*} a_{n} b\right)=\left(b \psi b^{*}\right)\left(a_{n}\right) \rightarrow\left\|b \psi b^{*}\right\|=\left(b \psi b^{*}\right)(1)=$ $\psi\left(b^{*} b\right)$ when $n \rightarrow \infty$. Using Assertion 1 we see that $b^{*} a_{n} b$ converges in norm to $b^{*} b$. Therefore

$$
\left\|\left(1-a_{n}\right)^{1 / 2} b\right\|^{2}=\left\|b^{*}\left(1-a_{n}\right) b\right\|=\left\|b^{*} a_{n} b-b^{*} b\right\| \rightarrow 0 .
$$

and

$$
\left\|\left(1-a_{n}\right) b\right\| \leq\left\|\left(1-a_{n}\right)^{1 / 2}\right\|\left\|\left(1-a_{n}\right)^{1 / 2} b\right\| \rightarrow 0,
$$

for $\left\|\left(1-a_{n}\right)^{1 / 2}\right\| \leq 1$ for all $n$. It shows that $a_{n} b$ converges to $b$ in norm.

Similarly, we get a proof that the sequence $\left\{b a_{n}\right\}_{n \in \mathbb{N}}$ converges to $b$ in norm. This proves the strict convergence of the sequence $\left\{a_{n}\right\}_{n \in \mathbb{N}}$ to $1 \in M(A)$.

The following proposition is a consequence of the right invariance of the Haar weight.

Proposition 3.2. Let $\left\{e_{n}\right\}_{n \in \mathbb{N}}$ be an orthonormal basis of the Hilbert space $\mathcal{H}$. Then for any $x, y$ belonging to the range of the GNS-mapping $\eta$, we have

$$
\sum_{n=1}^{\infty} W\left(e_{n}, x\right)^{*} W\left(e_{n}, y\right)=(x \mid y) 1,
$$

where the series converges with respect to the strict topology on $M(A)$.

Proof. Let $\omega \in A_{+}^{*}$ and $\left(\mathcal{H}_{\omega}, \pi_{\omega}, \Omega_{\omega}\right)$ be the GNS-triple associated with $\omega$. To proceed our computation we choose an orthonormal basis $\left\{\zeta_{n}\right\}_{n \in \mathbb{N}}$ in $\mathcal{H}_{\omega}$. Then for any $x \in \mathcal{H}$ we have

$$
\begin{aligned}
\omega\left(W\left(e_{n}, x\right)^{*} W\left(e_{n}, x\right)\right) & =\left\|\pi_{\omega}\left(W\left(e_{n}, x\right)\right) \Omega_{\omega}\right\|^{2}=\sum_{k=1}^{\infty}\left|\left(\zeta_{k} \mid \pi_{\omega}\left(W\left(e_{n}, x\right)\right) \Omega_{\omega}\right)\right|^{2} \\
& =\sum_{k=1}^{\infty}\left|\varphi_{k}\left(W\left(e_{n}, x\right)\right)\right|^{2}=\sum_{k=1}^{\infty}\left|\left(e_{n} \mid W_{\varphi_{k}} x\right)\right|^{2},
\end{aligned}
$$

where $\varphi_{k}$ are linear functionals on $A$ introduced by the formula

$$
\varphi_{k}(a):=\left(\zeta_{k} \mid \pi_{\omega}(a) \Omega_{\omega}\right)
$$

for all $a \in A$ and $k \in \mathbb{N}$. Summing over $n=1,2, \ldots, N$ and setting $N \rightarrow \infty$, we obtain

$$
\lim _{N \rightarrow \infty} \omega\left(\sum_{n=1}^{N} W\left(e_{n}, x\right)^{*} W\left(e_{n}, x\right)\right)=\sum_{n=1}^{\infty} \sum_{k=1}^{\infty}\left|\left(e_{n} \mid W_{\varphi_{k}} x\right)\right|^{2}=\sum_{k=1}^{\infty}\left\|W_{\varphi_{k}} x\right\|^{2} .
$$


Assume now that $x=\eta(a)$, where $a \in \mathcal{D}(\eta)$. Then the right hand side of the above relation equals to

$$
\begin{aligned}
\sum_{k=1}^{\infty}\left\|W_{\varphi_{k}} x\right\|^{2} & =\sum_{k=1}^{\infty}\left\|\eta\left(\varphi_{k} * a\right)\right\|^{2}=\sum_{k=1}^{\infty} h\left(\left(\varphi_{k} * a\right)^{*}\left(\varphi_{k} * a\right)\right) \\
& =\lim _{K \rightarrow \infty} h\left(\sum_{k=1}^{K}\left(\varphi_{k} * a\right)^{*}\left(\varphi_{k} * a\right)\right) .
\end{aligned}
$$

It turns out below (see the last part of the proof) that

$$
\left\|\sum_{k=1}^{K}\left(\varphi_{k} * a\right)^{*}\left(\varphi_{k} * a\right)-\omega *\left(a^{*} a\right)\right\| \rightarrow 0 \quad \text { as } \quad K \rightarrow \infty .
$$

Using the lower semicontinuity and the right invariance of $h$, we obtain

$$
\begin{aligned}
\lim _{N \rightarrow \infty} \omega\left(\sum_{n=1}^{N} W\left(e_{n}, x\right)^{*} W\left(e_{n}, x\right)\right) & =h\left(\omega *\left(a^{*} a\right)\right) \\
& =\|\omega\| h\left(a^{*} a\right)=\|\omega\|(x \mid x) .
\end{aligned}
$$

This result holds for any $\omega \in A_{+}^{*}$. By Assertion 2 of Lemma 3.1 we see that the series $\sum_{n=1}^{\infty} W\left(e_{n}, x\right)^{*} W\left(e_{n}, x\right)$ is strictly converging to $(x \mid x) 1$. To obtain (3.1) in full generality, we apply the polarization argument.

To end the proof we have to show (3.2). Let $\psi \in A_{+}^{*},\left(\mathcal{H}_{\psi}, \pi_{\psi}, \Omega_{\psi}\right)$ be the GNS-triple associated with $\psi$ and $\left\{\xi_{m}\right\}_{m \in \mathbb{N}}$ be an orthonormal basis in $\mathcal{H}_{\psi}$. Then

$$
\begin{aligned}
\psi\left(\omega *\left(a^{*} a\right)\right) & =(\psi \otimes \omega)\left(\delta\left(a^{*} a\right)\right)=\left\|\left(\pi_{\psi} \otimes \pi_{\omega}\right)(\delta(a))\left(\Omega_{\psi} \otimes \Omega_{\omega}\right)\right\|^{2} \\
& =\sum_{m, k=1}^{\infty}\left|\left(\xi_{m} \otimes \zeta_{k} \mid\left(\pi_{\psi} \otimes \pi_{\omega}\right)(\delta(a))\left(\Omega_{\psi} \otimes \Omega_{\omega}\right)\right)\right|^{2} \\
& =\sum_{m, k=1}^{\infty} \mid\left(\xi_{m}\left|\pi_{\psi}\left(\left(\mathrm{id} \otimes \varphi_{k}\right)(\delta(a)) \Omega_{\psi}\right)\right|^{2}=\sum_{k=1}^{\infty}\left\|\pi_{\psi}\left(\varphi_{k} * a\right) \Omega_{\psi}\right\|^{2}\right. \\
& =\sum_{k=1}^{\infty} \psi\left(\left(\varphi_{k} * a\right)^{*}\left(\varphi_{k} * a\right)\right)=\lim _{K \rightarrow \infty} \psi\left(\sum_{k=1}^{K}\left(\varphi_{k} * a\right)^{*}\left(\varphi_{k} * a\right)\right) .
\end{aligned}
$$

Now (3.2) follows from Assertion 1 of Lemma 3.1

Proposition 3.3. Let $\left\{e_{n}\right\}_{n \in \mathbb{N}}$ be an orthonormal basis of the Hilbert space $\mathcal{H}$. Then for any $x, y$ such that $J x$ and $J y$ belong to the range of $\eta$, we have

$$
\sum_{n=1}^{\infty} W\left(x, e_{n}\right) W\left(y, e_{n}\right)^{*}=(x \mid y) 1
$$

with respect to the strict topology.

Proof. Replacing in the previous Proposition $\left\{e_{n}\right\}_{n \in \mathbb{N}}, x$ and $y$ by $\left\{J e_{n}\right\}_{n \in \mathbb{N}}, J y$ and $J x$ respectively, we obtain

$$
\sum_{n=1}^{\infty} W\left(J e_{n}, J y\right)^{*} W\left(J e_{n}, J x\right)=(J y \mid J x) 1=(x \mid y) 1 .
$$

The unitary antipode $R$ can be extended to the multiplier algebra $M(A)$ and the extension denoted by the same symbol is continuous with respect to the strict topology. Remembering that the above series is strictly converging, we have

$$
\sum_{n=1}^{\infty} R\left(W\left(J e_{n}, J x\right)\right) R\left(W\left(J e_{n}, J y\right)\right)^{*}=(x \mid y) 1 .
$$

Thus (3.3) follows. (cf. Second formula of Proposition 2.2)

Remark 3.4. In what follows, relations (3.1) and (3.3) will be used to prove the unitarity of the Kac-Takesaki operator. For this purpose, it is sufficient to know that the series (3.1) and (3.3) converge weakly. However to prove (3.3) by using (3.1) we have to use strict topology ; at the moment the continuity of $R$ with respect to the weak operator topology is not established yet. 
The Kac-Takesaki operator $W$ is introduced by the following theorem. It plays a role of the right regular representation for the "quantum group" associated with the weighted Hopf $C^{*}$-algebra. We recall that, for any $x, y \in \mathcal{H}, \omega_{x, y}$ denotes the linear functional on $\mathcal{L}(\mathcal{H})$ defined by $\omega_{x, y}(a)=$ $(x \mid a y)$.

Theorem 3.5. There exists a unique unitary operator $W$ acting on $\mathcal{H} \otimes \mathcal{H}$ such that

$$
\begin{gathered}
(x \otimes z \mid W(y \otimes u))=(z \mid W(x, y) u), \\
\left(\omega_{x, y} \otimes \mathrm{id}\right)(W)=W(x, y), \\
(\operatorname{id} \otimes \varphi) W=W_{\varphi}
\end{gathered}
$$

for any $x, y, z, u \in \mathcal{H}$ and $\varphi \in \mathcal{L}(\mathcal{H})_{*}$. On the right hand side of the last relation $\varphi \in A^{*}$ denotes the restriction of $\varphi \in \mathcal{L}(\mathcal{H})_{*}$ to $A$. Each of the above equations determines $W$ uniquely.

Proof. Clearly (3.4) and (3.5) are equivalent. The computation

$$
\left(z\left|\left(\operatorname{id} \otimes \omega_{y, x}\right)(W)\right| u\right)=(z \otimes y \mid W(u \otimes x))=(y \mid W(z, u) x)=\left(z \mid W_{\omega_{y, x}} u\right)
$$

shows the equivalence of (3.4) and (3.6). The uniqueness of $W$ is obvious.

Let $D_{0}$ be the range of the GNS-mapping $\eta$ and $D_{1}=J D_{0}$. Then $D_{0}$ and $D_{1}$ are dense linear subsets of $\mathcal{H}$. We choose an orthonormal basis $\left\{e_{n}\right\}_{n \in \mathbb{N}}$ in $\mathcal{H}$. For any $y \in D_{0}, x \in D_{1}, z, u \in A \mathcal{H}$ we set

and

$$
W(y \otimes u):=\sum_{n=1}^{\infty} e_{n} \otimes W\left(e_{n}, y\right) u
$$

$$
W^{\dagger}(x \otimes z):=\sum_{n=1}^{\infty} e_{n} \otimes W\left(x, e_{n}\right)^{*} z .
$$

Using (3.1) and (3.3) one can easily show that the series on the right hand sides are norm converging in $\mathcal{H} \otimes \mathcal{H}$. Clearly the above formulae introduce linear mappings $W: D_{0} \otimes_{\text {alg }} A \mathcal{H} \rightarrow \mathcal{H} \otimes \mathcal{H}$ and $W^{\dagger}: D_{1} \otimes_{\text {alg }} A \mathcal{H} \rightarrow \mathcal{H} \otimes \mathcal{H}$. Using (3.1) and (3.3) once more, we can show that these mappings are isometries. Extending these mappings by continuity, we obtain isometric operators $W$ and $W^{\dagger}$ defined on the whole $\mathcal{H} \otimes \mathcal{H}$.

To show that $W$ is unitary, it is sufficient to prove that the adjoint $W^{*}$ is an isometry. We claim that $W^{*}=W^{\dagger}$. Indeed, for any $y \in D_{0}, x \in D_{1}, z, u \in A \mathcal{H}$, we have

and

$$
\begin{aligned}
(x \otimes z \mid W(y \otimes u)) & =\sum_{n=1}^{\infty}\left(x \otimes z \mid e_{n} \otimes W\left(e_{n}, y\right) u\right)=\sum_{n=1}^{\infty}\left(x \mid e_{n}\right)\left(z \mid W\left(e_{n}, y\right) u\right) \\
& =\left(z \mid W\left(\sum_{n=1}^{\infty} e_{n}\left(e_{n} \mid x\right), y\right) u\right)=(z \mid W(x, y) u)
\end{aligned}
$$

$$
\begin{aligned}
\left(W^{\dagger}(x \otimes z) \mid y \otimes u\right) & =\sum_{n=1}^{\infty}\left(e_{n} \otimes W\left(x, e_{n}\right)^{*} z \mid y \otimes u\right) \\
& =\sum_{n=1}^{\infty}\left(e_{n} \mid y\right)\left(z \mid W\left(x, e_{n}\right) u\right) \\
& =\left(z \mid W\left(x, \sum_{n=1}^{\infty} e_{n}\left(e_{n} \mid y\right)\right) u\right)=(z \mid W(x, y) u) .
\end{aligned}
$$

By the way we proved (3.4).

We shall use the leg numbering notation [1], for example:

$$
\left(x_{1} \otimes x_{2} \otimes x_{3} \mid W_{13}\left(y_{1} \otimes y_{2} \otimes y_{3}\right)\right)=\left(x_{1} \otimes x_{3} \mid W\left(y_{1} \otimes y_{3}\right)\right)\left(x_{2} \mid y_{2}\right)
$$

for $x_{j}, y_{j} \in \mathcal{H}$ for $j=1,2,3$. We shall also use an exact vector presentation (see Appendix B) of the GNS map $\eta$ :

$$
\eta(a)=\sum_{n \in \mathbb{N}} a \Omega_{n}
$$


for any $a \in \mathcal{D}(\eta)$, where $\Omega_{n} \in \mathcal{H}$ for $n=1,2, \ldots, A \Omega_{n} \perp A \Omega_{m}$ whenever $n \neq m$ and $\mathcal{D}(\eta)$ coincides with the set of all $a \in A$ such that the above series is convergent. Moreover $\sum_{n \in \mathbb{N}} A \Omega_{n}$ is norm dense in $\mathcal{H}$, this is because the range of $\eta$ is dense.

In the following Proposition we collect basic properties of the Kac-Takesaki operator.

\section{Proposition 3.6.}

(1) The coproduct $\delta$ is implemented by $W$ :

$$
\delta(a)=W(a \otimes 1) W^{*}
$$

for any $a \in A$.

(2) The pentagonal equation $W_{12} W_{13} W_{23}=W_{23} W_{12}$ holds. In other words

$$
(\mathrm{id} \otimes \delta) W=W_{12} W_{13} .
$$

(3) The operator $W$ commutes with the operators $Q \otimes Q$ and $\Delta \otimes Q^{2}$ :

$$
W^{*}(Q \otimes Q) W=Q \otimes Q
$$

$$
W^{*}\left(\Delta \otimes Q^{2}\right) W=\Delta \otimes Q^{2}
$$

Proof.

Ad 1. For any $x, y, z \in \mathcal{H}$ and $a \in \mathcal{D}(\eta)$ we have

$$
\begin{aligned}
(x \otimes z \mid W(\eta(a) \otimes y)) & =\left(x \mid\left(\mathrm{id} \otimes \omega_{z, y}\right)(W) \eta(a)\right)=\left(x \mid W_{\omega_{z, y}} \eta(a)\right) \\
& =\left(x \mid \eta\left(\omega_{z, y} * a\right)\right)=\sum_{n \in \mathbb{N}}\left(x \mid\left(\omega_{z, y} * a\right) \Omega_{n}\right) .
\end{aligned}
$$

Let $p_{n}$ be the projection onto the closure of $A \Omega_{n}$. Then $p_{n} \in A^{\prime}$ and $\sum_{n \in \mathbb{N}} p_{n}=1$. Replacing $x$ by $p_{n} x$, we obtain:

$$
\begin{aligned}
\left(x \otimes z \mid\left(p_{n} \otimes 1\right) W(\eta(a) \otimes y)\right) & =\left(x \mid\left(\omega_{z, y} * a\right) \Omega_{n}\right) \\
& =\left(x \mid\left(\left(\operatorname{id} \otimes \omega_{z, y}\right)(\delta(a))\right) \Omega_{n}\right)=\left(x \otimes z \mid \delta(a)\left(\Omega_{n} \otimes y\right)\right) .
\end{aligned}
$$

Therefore

It shows that

$$
\left(p_{n} \otimes 1\right) W(\eta(a) \otimes y)=\delta(a)\left(\Omega_{n} \otimes y\right) .
$$

$$
W(\eta(a) \otimes y)=\sum_{n \in \mathbb{N}} \delta(a)\left(\Omega_{n} \otimes y\right),
$$

where the right hand side converges in norm.

Now for any $a \in A, b \in \mathcal{D}(\eta)$ and $y \in \mathcal{H}$ we have

$$
\begin{aligned}
\delta(a) W(\eta(b) \otimes y) & =\delta(a) \sum_{n \in \mathbb{N}} \delta(b)\left(\Omega_{n} \otimes y\right) \\
& =\sum_{n \in \mathbb{N}} \delta(a b)\left(\Omega_{n} \otimes y\right)=W(\eta(a b) \otimes y)=W(a \otimes 1)(\eta(b) \otimes y) .
\end{aligned}
$$

Therefore $\delta(a) W=W(a \otimes 1)$ and $\delta(a)=W(a \otimes 1) W^{*}$.

Ad 2. Let $\varphi, \psi \in \mathcal{L}(\mathcal{H})_{*}$. For any $a \in \mathcal{L}(\mathcal{H})$ we set

$$
\rho(a)=(\varphi \otimes \psi)\left(W(a \otimes 1) W^{*}\right) .
$$

Then $\rho \in \mathcal{L}(\mathcal{H})_{*}$. Moreover

$$
(\mathrm{id} \otimes \rho)(W)=(\mathrm{id} \otimes \varphi \otimes \psi)\left(W_{23} W_{12} W_{23}^{*}\right) .
$$

If $a \in A$, then by the previous assertion $\rho(a)=(\varphi \otimes \psi)(\delta(a))=(\varphi * \psi)(a)$. It shows that the restriction of $\rho$ to $A$ coincides with $\varphi * \psi$. Using the first assertion we have

$$
(\mathrm{id} \otimes \rho)(W)=W_{\varphi * \psi}=W_{\varphi} W_{\psi}=(\mathrm{id} \otimes \varphi \otimes \psi)\left(W_{12} W_{13}\right) .
$$

Comparing this with (3.12), we see that $W_{23} W_{12} W_{23}^{*}=W_{12} W_{13}$ and the pentagonal equation follows. Taking into account (3.7), we obtain (3.8).

Ad 3. Let $\varphi \in \mathcal{L}(\mathcal{H})_{*}, t \in \mathbb{R}$ and $\varphi_{t}(a):=\varphi\left(Q^{2 i t} a Q^{-2 i t}\right)$ for any $a \in \mathcal{L}(\mathcal{H})$. By (2.2), $\varphi_{t}$ restricted to $A$ coincides with $\varphi \circ \tau_{t}$. Therefore

$$
(\mathrm{id} \otimes \varphi)\left(\left(1 \otimes Q^{2 i t}\right) W\left(1 \otimes Q^{-2 i t}\right)\right)=\left(\mathrm{id} \otimes \varphi_{t}\right)(W)=W_{\varphi^{\circ} \tau_{t}} .
$$


Using now Proposition 2.5 and Lemma 2.8 we obtain:

$$
\begin{gathered}
W_{\varphi^{\mathrm{o}} \tau_{t}}=\Delta^{-i t} W_{\varphi} \Delta^{i t}=(\mathrm{id} \otimes \varphi)\left(\left(\Delta^{-i t} \otimes 1\right) W\left(\Delta^{i t} \otimes 1\right)\right) \\
W_{\varphi^{\mathrm{o}} \tau_{t}}=Q^{-2 i t} W_{\varphi} Q^{2 i t}=(\mathrm{id} \otimes \varphi)\left(\left(Q^{-2 i t} \otimes 1\right) W\left(Q^{2 i t} \otimes 1\right)\right) .
\end{gathered}
$$

Comparing these with the previous relation, we get

$$
\begin{aligned}
\left(1 \otimes Q^{2 i t}\right) W\left(1 \otimes Q^{-2 i t}\right) & =\left(\Delta^{-i t} \otimes 1\right) W\left(\Delta^{i t} \otimes 1\right) \\
& =\left(Q^{-2 i t} \otimes 1\right) W\left(Q^{2 i t} \otimes 1\right) .
\end{aligned}
$$

It shows that $\Delta^{i t} \otimes Q^{2 i t}$ and $Q^{2 i t} \otimes Q^{2 i t}$ commute with $W$ and our statement follows.

Combining (1.9) with (3.6) we obtain

$$
(\mathrm{id} \otimes \varphi \circ \kappa) W=(\mathrm{id} \otimes \varphi)\left(W^{*}\right)
$$

for any $\varphi \in A^{*}$ such that $\varphi \circ \kappa \in A^{*}$.

Let us recall the basic setup of the theory of multiplicative unitaries discussed in 1 , 34. Let $\mathcal{H}$ be a separable Hilbert space. A unitary operator $W$ acting on $\mathcal{H} \otimes \mathcal{H}$ is called a multiplicative unitary if it satisfies the pentagonal equation $W_{23} W_{12}=W_{12} W_{13} W_{23}$. Let $J$ be a conjugate linear bijection $\mathcal{H} \ni x \mapsto J x \in \mathcal{H}$ with $(J x \mid J y)=(y \mid x)$. A multiplicative unitary $W$ is said to be manageable if there exist a strictly positive self-adjoint operator $Q$ acting on $\mathcal{H}$ and a unitary operator $\widetilde{W}$ acting on $\mathcal{H} \otimes \mathcal{H}$ such that

(1) $W^{*}(Q \otimes Q) W=Q \otimes Q$,

(2) $\left(x_{1} \otimes x_{2} \mid W\left(y_{1} \otimes y_{2}\right)\right)=\left(J y_{1} \otimes Q x_{2} \mid \widetilde{W}\left(J x_{1} \otimes Q^{-1} y_{2}\right)\right)$ for all $x_{1}, y_{1} \in \mathcal{H}, x_{2} \in \mathcal{D}(Q)$ and $y_{2} \in \mathcal{D}\left(Q^{-1}\right)$.

Clearly the definition and the operator $Q$ are independent of the choice of $J$.

Proposition 3.7. The Kac-Takesaki operator $W$ is manageable.

Proof. Let $J$ be the involutive antiunitary introduced in the paragraph preceding Lemma 2.1] We shall prove that the above two conditions are satisfied by the operator $Q$ introduced in (2.1) and $\widetilde{W}=W^{*}$. We already know that $Q$ is strictly positive and that $Q \otimes Q$ commutes with $W$.

Let $x, y \in \mathcal{H}$. By Proposition $2.4 W(x, y) \in \mathcal{D}(\kappa)$ and $\kappa(W(x, y))=W(y, x)^{*}$. Taking into account the definition of $\kappa$ and using the second formula of Proposition 2.2 we see that $W(x, y) \in$ $\mathcal{D}\left(\tau_{i / 2}\right)$ and

$$
\tau_{i / 2}(W(x, y))=R\left(W(y, x)^{*}\right)=W(J x, J y)^{*} .
$$

Let $z, u \in \mathcal{H}$. Then for any $t \in \mathbb{R}$ we have:

$$
\begin{aligned}
\left(Q^{2 i t} z \mid \tau_{t}(W(x, y)) Q^{2 i t} u\right) & =\left(z \mid Q^{-2 i t} \tau_{t}(W(x, y)) Q^{2 i t} u\right) \\
& =(z \mid W(x, y) u)
\end{aligned}
$$

Assume that $z \in \mathcal{D}(Q)$ and $u \in \mathcal{D}\left(Q^{-1}\right)$. Making the holomorphic continuation up to the point $t=i / 2$, we obtain

Therefore

$$
\left(Q z \mid W(J x, J y)^{*} Q^{-1} u\right)=(z \mid W(x, y) u)
$$

$$
\begin{aligned}
(x \otimes z \mid W(y \otimes u)) & =(z \mid W(x, y) u)=\overline{\left(Q^{-1} u \mid W(J x, J y) Q z\right)} \\
& =\overline{\left(J x \otimes Q^{-1} u \mid W(J y \otimes Q z)\right)}=\left(J y \otimes Q z \mid W^{*}\left(J x \otimes Q^{-1} u\right)\right) .
\end{aligned}
$$

Let us recall the properties of manageable multiplicative unitaries obtained in [1, 34.

Theorem 3.8. Let $\mathcal{H}$ be a Hilbert space and $W$ be a manageable multiplicative unitary on $\mathcal{H} \otimes \mathcal{H}$. We set:

$$
\begin{aligned}
& A=\left\{(\varphi \otimes \mathrm{id})(W): \varphi \in \mathcal{L}(\mathcal{H})_{*}\right\}^{\text {norm closure }}, \\
& \widehat{A}=\left\{(\mathrm{id} \otimes \varphi)\left(W^{*}\right): \varphi \in \mathcal{L}(\mathcal{H})_{*}\right\}^{\text {norm closure }} .
\end{aligned}
$$

Then

(1) $A$ and $\widehat{A}$ are $C^{*}$-algebras acting non degenerately on $\mathcal{H}$.

(2) The operator $W$ is an element of the multiplier algebra $M(\widehat{A} \otimes A)$. 
(3) There exists a unique $\delta \in \operatorname{Mor}(A, A \otimes A)$ such that $(\mathrm{id} \otimes \delta)(W)=W_{12} W_{13}$. The pair $(A, \delta)$ is a proper $C^{*}$-bialgebra with the cancellation property. The comultiplication is given by the formula

$$
\delta(a)=W(a \otimes 1) W^{*}
$$

for all $a \in A$.

(4) There exists a unique closed linear mapping $\kappa: A \rightarrow A$ ( $A$ is treated as a Banach space) for which the set $\left\{(\varphi \otimes \mathrm{id})(W): \varphi \in \mathcal{L}(\mathcal{H})_{*}\right\}$ is a core and

$$
\kappa((\varphi \otimes \mathrm{id})(W))=(\varphi \otimes \mathrm{id})\left(W^{*}\right)
$$

for any $\varphi \in \mathcal{L}(\mathcal{H})_{*}$. Furthermore the set $\mathcal{D}(\kappa)$ is a subalgebra of $A$ and the mapping $\kappa$ : $\mathcal{D}(\kappa) \rightarrow A$ is antimultiplicative. Moreover, $\kappa(\mathcal{D}(\kappa))=\left\{a: a^{*} \in \mathcal{D}(\kappa)\right\}$ and $\kappa\left(\kappa(a)^{*}\right)^{*}=a$ for $a \in \mathcal{D}(\kappa)$.

(5) The operator $\kappa$ admits the following polar decomposition:

$$
\kappa=R^{\circ} \tau_{i / 2}
$$

where $\tau_{i / 2}$ is the analytic generator of a one parameter group $\left\{\tau_{t}\right\}_{t \in \mathbb{R}}$ of automorphisms of the $C^{*}$-algebra $A$ and $R$ is an involutive antiautomorphism of $A$ commuting with $\tau_{t}$ for all $t \in \mathbb{R}$. In particular, $\mathcal{D}(\kappa)=\mathcal{D}\left(\tau_{i / 2}\right)$. In this case $R$ and $\left\{\tau_{t}\right\}_{t \in \mathbb{R}}$ are uniquely determined.

(6) We have

$$
\delta \circ \tau_{t}=\left(\tau_{t} \otimes \tau_{t}\right) \circ \delta \quad \text { for } \quad t \in \mathbb{R} ; \quad \delta \circ R=\sigma o(R \otimes R) \circ \delta .
$$

(7) $R$ is normal, i.e. continuous with respect to the $\sigma$-weak topology on $A$.

(8) Let $\widetilde{W}$ and $Q$ be the operators in the definition of manageability for $W$. Then they satisfy $\tau_{t}(a)=Q^{2 i t} a Q^{-2 i t}$ for $t \in \mathbb{R}$ and $W^{\top \otimes R}=\widetilde{W}^{*}$, where $b^{\top}=J b^{*} J$.

We shall apply this theorem to the Kac-Takesaki operator $W$ introduced in Theorem 3.5 We know that the set $\left\{\omega_{x, y}: x, y \in \mathcal{H}\right\}$ is linearly dense in $\mathcal{L}(\mathcal{H})_{*}$. Inserting in 3.15) $\varphi=\omega_{x, y}$ and using (3.5) we obtain

$$
A=\{W(x, y): x, y \in \mathcal{H}\}^{\text {closed linear envelope }} .
$$

Now Proposition 1.12 shows that the algebra (3.15) coincides with the original algebra $A$ that we started with. Comparing (3.17) with (3.7), we see that new $\delta$ coincides with the original one.

Inserting in (3.18) $\varphi=\omega_{x, y}$ for $x, y \in \mathcal{H}$, we obtain

$$
\begin{aligned}
\kappa(W(x, y)) & =\kappa\left(\left(\omega_{x, y} \otimes \mathrm{id}\right)(W)\right)=\left(\omega_{x, y} \otimes \mathrm{id}\right)\left(W^{*}\right) \\
& =\left(\omega_{y, x} \otimes \mathrm{id}\right)(W)^{*}=W(y, x)^{*} .
\end{aligned}
$$

Comparing now Statement 4 of Theorem 1.13 and Corollary 2.4 with Assertion 4 of the above theorem, we see that new $\kappa$ coincides with the old one (they have the same core on which they act in the same way). By the uniqueness of the polar decomposition, we see that the new $R$ and $\tau$ coincide with the old ones.

Now we are ready to construct the dual weighted Hopf $C^{*}$-algebra $(\widehat{A}, \widehat{\delta})$ associated with our weighted Hopf $C^{*}$-algebra $(A, \delta)$. Let $\Sigma \in \mathcal{L}(\mathcal{H} \otimes \mathcal{H})$ be the flip map : $\Sigma(x \otimes y)=y \otimes x$ for all $x, y \in \mathcal{H}$. According to the general theory [1, 34, the operator

$$
\widehat{W}=\Sigma W^{*} \Sigma
$$

is a manageable multiplicative unitary. We shall apply Theorem 3.8 to $\widehat{W}$. Clearly, replacing $W$ by $\widehat{W}$, we interchange the roles of $A$ and $\widehat{A}$. The comultiplication $\widehat{\delta} \in \operatorname{Mor}(\widehat{A}, \widehat{A} \otimes \widehat{A})$ related to $\widehat{W}$ satisfies the relation (id $\otimes \widehat{\delta}) \widehat{W}=\widehat{W}_{12} \widehat{W}_{13}$ and is given by the formula $\widehat{\delta}(b)=\widehat{W}(b \otimes 1) \widehat{W}^{*}$ where $b$ runs over $\widehat{A}$. Using (3.20), we obtain

$$
(\widehat{\delta} \otimes \mathrm{id}) W^{*}=W_{13}^{*} W_{23}^{*}
$$

and

$$
\widehat{\delta}(b)=\Sigma W^{*}(1 \otimes b) W \Sigma .
$$

By Theorem $3.8(\widehat{A}, \widehat{\delta})$ is a proper $\mathrm{C}^{*}$-bialgebra with the cancellation property. It will be proved in Section $\mathbf{7}$ that $(\widehat{A}, \widehat{\delta})$ is a weighted Hopf $C^{*}$-algebra. We say that $(\widehat{A}, \widehat{\delta})$ is the dual of $(A, \delta)$. 
Assertion 4 of Theorem 3.8 provide us with the closed linear mapping $\widehat{\kappa}$ acting on $\widehat{A}$ such that the set $\left\{(\operatorname{id} \otimes \varphi)\left(W^{*}\right): \varphi \in \mathcal{L}(\mathcal{H})_{*}\right\}$ is core for $\widehat{\kappa}$ and

$$
\widehat{\kappa}\left((\mathrm{id} \otimes \varphi)\left(W^{*}\right)\right)=(\mathrm{id} \otimes \varphi)(W) .
$$

Clearly, $\widehat{\kappa}$ is the antipode related to $(\widehat{A}, \widehat{\delta})$. By Assertion 5,

$$
\widehat{\kappa}=\widehat{R} \circ \widehat{\tau}_{i / 2},
$$

where $\left\{\widehat{\tau}_{t}\right\}_{t \in \mathbb{R}}$ is a one parameter group of automorphisms of $\widehat{A}$ and $\widehat{R}$ is an involutive antiautomorphism of $\widehat{A}$ which commutes with $\widehat{\tau}_{t}$ for $t \in \mathbb{R}$. Clearly, $\left\{\widehat{\tau}_{t}\right\}_{t \in \mathbb{R}}$ and $\widehat{R}$ are the scaling group and the unitary antipode related to $(\widehat{A}, \widehat{\delta})$.

According to Statement 3 of Proposition 1.4 of [34], $\widehat{W}$ is manageable. Inspecting the proof of this statement, we see that the operators $\widehat{Q}$ and $\widehat{\widehat{W}}$ entering the definition of the manageability are given by the formulae

$$
\widehat{Q}=Q, \quad \widetilde{\widehat{W}}=\left(\Sigma \widetilde{W}^{*} \Sigma\right)^{\top \otimes \top} .
$$

We know (cf. the proof of Proposition 3.7) that $\widetilde{W}=W^{*}$. Therefore

$$
\widetilde{\widehat{W}}=(\Sigma W \Sigma)^{\top \otimes \top}=(J \otimes J) \Sigma W^{*} \Sigma(J \otimes J) .
$$

Using now Assertion 8 of Theorem 3.8, we obtain

$$
\widehat{\tau}_{t}(b)=Q^{2 i t} b Q^{-2 i t}
$$

for any $t \in \mathbb{R}$ and $b \in \widehat{A}$ and

$$
\widehat{W}^{\top \otimes \widehat{R}}=(J \otimes J) \Sigma W \Sigma(J \otimes J) .
$$

Applying the flip and ${ }^{*}$-conjugation to both sides, we obtain

$$
W^{\widehat{R} \otimes \top}=(J \otimes J) W^{*}(J \otimes J)=W^{\top \otimes \top} .
$$

Therefore

$$
W^{\widehat{R}^{\circ} \mathrm{T} \otimes \mathrm{id}}=W .
$$

Let $(A, \delta)$ be a $\mathrm{C}^{*}$-bialgebra. In concrete cases one of the difficult task is to show that a given weight $h$ on $A$ is right invariant and that the strong right invariance holds. To this end we shall use the following

Theorem 3.9. Let $(A, \delta)$ be a proper $C^{*}$-bialgebra with the cancellation property, $\kappa$ be a closed operator acting on the Banach space $A$ admitting the polar decomposition

$$
\kappa=R \tau_{i / 2},
$$

where $\tau_{i / 2}$ is the analytic generator of a one parameter group $\left\{\tau_{t}\right\}_{t \in \mathbb{R}}$ of automorphisms of the $C^{*}$-algebra $A$ and $R$ is an involutive antiautomorphism of $A$ commuting with $\tau_{t}$ for all $t \in \mathbb{R}$ and let $h$ be a strictly faithful locally finite lower semicontinuous weight on $A$ such that ho $\tau_{t}=\lambda^{t} h$ for some fixed $\lambda>0$ and for all $t \in \mathbb{R}$. We shall use the $G N S$ triple $(\mathcal{H}, \pi, \eta)$ related to $h$ and identify $A$ with $\pi(A) \subset \mathcal{L}(\mathcal{H})$.

Assume that a unitary element $W \in M(\mathcal{K}(\mathcal{H}) \otimes A)$ satisfies the following two conditions:

1. For any $a \in \mathcal{D}(\eta)$ and any $\varphi \in A^{*}$ we have:

$$
\left.\begin{array}{c}
\varphi * a \in \mathcal{D}(\eta) \text { and } \\
\eta(\varphi * a)=(\mathrm{id} \otimes \varphi) W \eta(a),
\end{array}\right\}
$$

2. For any $\varphi \in A^{*}$ such that $\varphi \circ \in A^{*}$ we have:

$$
\left(\mathrm{id} \otimes \varphi^{\circ} \kappa\right) W=(\mathrm{id} \otimes \varphi)\left(W^{*}\right) .
$$

Then $(A, \delta)$ is a weighted Hopf $C^{*}$-algebra and $h, R, \tau$ and $W$ coincide with the right Haar weight, the unitary antipode, the scaling group and the Kac-Takesaki operator related to $(A, \delta)$.

Proof. We have to show that $h$ is right invariant and that the strong right invariance holds. We shall follow the proof of Assertion 1 of Proposition 3.6. Let

$$
\eta(b)=\sum_{n \in \mathbb{N}} b \Omega_{n}
$$


be an exact vector presentation of the GNS map $\eta$. Then

$$
h(b)=\sum_{n \in \mathbb{N}}\left(\Omega_{n} \mid b \Omega_{n}\right)
$$

for any $b \in A_{+}$. Take $x, y, z \in \mathcal{H}$ and $a \in \mathcal{D}(\eta)$. Then, by (3.24), we have

$$
\begin{aligned}
(x \otimes z|W| \eta(a) \otimes y) & =\left(x\left|\left(\operatorname{id} \otimes \omega_{z, y}\right) W\right| \eta(a)\right)=\left(x \mid \eta\left(\left(\operatorname{id} \otimes \omega_{z, y}\right) \delta(a)\right)\right) \\
& =\sum_{n \in \mathbb{N}}\left(x\left|\left(\operatorname{id} \otimes \omega_{z, y}\right) \delta(a)\right| \Omega_{n}\right)=\sum_{n \in \mathbb{N}}\left(x \otimes z|\delta(a)| \Omega_{n} \otimes y\right) .
\end{aligned}
$$

Therefore

$$
W(\eta(a) \otimes y)=\sum_{n \in \mathbb{N}} \delta(a)\left(\Omega_{n} \otimes y\right)
$$

Computing the norm of the both sides, we obtain:

$$
\begin{aligned}
\omega_{y, y}(1) h\left(a^{*} a\right) & =\|y\|^{2} h\left(a^{*} a\right)=\sum_{n \in \mathbb{N}}\left\|\delta(a)\left(\Omega_{n} \otimes y\right)\right\|^{2}=\sum_{n \in \mathbb{N}}\left(\Omega_{n} \otimes y\left|\delta\left(a^{*} a\right)\right| \Omega_{n} \otimes y\right) \\
& =\sum_{n \in \mathbb{N}}\left(\Omega_{n}\left|\omega_{y, y} *\left(a^{*} a\right)\right| \Omega_{n}\right)=h\left(\omega_{y, y} *\left(a^{*} a\right)\right) .
\end{aligned}
$$

In this way we proved that $\psi(1) h\left(a^{*} a\right)=h\left(\psi *\left(a^{*} a\right)\right)$ for all $\psi$ of the form $\omega_{y, y}(y \in \mathcal{H})$ i.e. for all $\psi \in A_{*+}$. We have to show that this formula holds for all $\psi \in A_{+}^{*}$.

Let $p_{n}$ be the projection onto the closure of $A \Omega_{n}$. Then $p_{n} \in A^{\prime}$ and $\sum_{n \in \mathbb{N}} p_{n}=1$. Replacing in (3.26), $x$ by $p_{n} x$, we obtain:

$$
\left(x \otimes z\left|\left(p_{n} \otimes 1\right) W\right| \eta(a) \otimes y\right)=\left(x \otimes z|\delta(a)| \Omega_{n} \otimes y\right) .
$$

Therefore

$$
\left(\omega_{x, \eta(a)} \otimes \mathrm{id}\right)\left[\left(p_{n} \otimes 1\right) W\right]=\left(\omega_{x, \Omega_{n}} \otimes \mathrm{id}\right) \delta(a) \in A .
$$

Let $\pi^{\prime}$ be a representation of $A$ acting on a Hilbert space $\mathcal{H}^{\prime}$. Then

$$
\left(\omega_{x, \eta(a)} \otimes \pi^{\prime}\right)\left[\left(p_{n} \otimes 1\right) W\right]=\left(\omega_{x, \Omega_{n}} \otimes \pi^{\prime}\right) \delta(a)
$$

and for any $z^{\prime}, y^{\prime} \in \mathcal{H}^{\prime}$ we have

$$
\left(x \otimes z^{\prime}\left|\left(p_{n} \otimes 1\right)\left(\mathrm{id} \otimes \pi^{\prime}\right) W\right| \eta(a) \otimes y^{\prime}\right)=\left(x \otimes z^{\prime}\left|\left(\mathrm{id} \otimes \pi^{\prime}\right) \delta(a)\right| \Omega_{n} \otimes y^{\prime}\right) .
$$

This way we showed that

$$
\left(p_{n} \otimes 1\right)\left(\mathrm{id} \otimes \pi^{\prime}\right) W\left(\eta(a) \otimes y^{\prime}\right)=\left(\mathrm{id} \otimes \pi^{\prime}\right) \delta(a)\left(\Omega_{n} \otimes y^{\prime}\right)
$$

and summing over $n$ we obtain

$$
\left(\mathrm{id} \otimes \pi^{\prime}\right) W\left(\eta(a) \otimes y^{\prime}\right)=\sum_{n}\left(\mathrm{id} \otimes \pi^{\prime}\right) \delta(a)\left(\Omega_{n} \otimes y^{\prime}\right)
$$

with the norm convergent sum on the right hand side. This relation holds for any $a \in \mathcal{D}(\eta)$. Computing the square of the norm of both sides, we obtain:

$$
(\eta(a) \mid \eta(a))\left(y^{\prime} \mid y^{\prime}\right)=\sum_{n}\left(\Omega_{n} \otimes y^{\prime}\left|\left(\mathrm{id} \otimes \pi^{\prime}\right) \delta\left(a^{*} a\right)\right| \Omega_{n} \otimes y^{\prime}\right) .
$$

For any $b \in A$ we set:

$$
\psi(b)=\left(y^{\prime} \mid \pi^{\prime}(b) y^{\prime}\right)
$$

Then formula (3.28) takes the form

$$
h\left(a^{*} a\right) \psi(1)=\sum_{n}\left(\Omega_{n}\left|(\operatorname{id} \otimes \psi) \delta\left(a^{*} a\right)\right| \Omega_{n}\right)=h\left(\psi *\left(a^{*} a\right)\right) .
$$

To end the proof of the right invariance we notice that any positive functional $\psi$ on $A$ is of the form (3.29) by the GNS construction.

We pass to the strong right invariance. It follows immediately from Theorem 1.13 (Equivalence of Statements 1 and 2). Therefore $(A, \delta)$ is a weighted Hopf $\mathrm{C}^{*}$-algebra. Formula (3.27) is identical with (3.11). It shows that $W$ is the Kac-Takesaki operator related to $(A, \delta)$.

The reader should notice that Assumption (3.24) was used only with $\varphi$ of the form $\omega_{z, y}$ where $z, y \in \mathcal{H}$. On the other hand the set of pairs $(\varphi, a) \in A^{*} \times \mathcal{D}(\eta)$ satisfying (3.24) is closed with 
respect to the norm topology on $A^{*}$ and the graph topology on $\mathcal{D}(\eta)$. This observation leads to the following

Remark 3.10. It is sufficient to verify condition (3.24) for all $\varphi$ in a norm dense subset of $A_{*}$ and for all $a$ in a core of $\eta$.

Taking into account the equivalence of Statements 2 and 3 of Theorem 1.13, we obtain

Remark 3.11. It is sufficient to verify condition (3.25) for all $\varphi$ from a weakly* dense $\tau$-invariant subset of $A^{*}$.

\section{Modular Structure for Haar Weight}

In what follows $W$ always denotes the Kac-Takesaki operator for $(A, \delta)$, and $A_{*}$ denotes the set of all $\sigma$-weakly continuous functionals on $A$. In this section, we investigate the Connes' RadonNikodym cocycle of the left invariant Haar weight $h^{L}:=h \circ R$ with respect to the right invariant Haar weight $h$. For this purpose, we have to work on the von Neumann algebra $M=A^{\prime \prime}$ which is the weak closure of the $C^{*}$-algebra $A$ acting on the GNS Hilbert space $\mathcal{H}$. By (2.2) and (3.7), the scaling automorphisms $\tau_{t}$ and the comultiplication $\delta$ are weakly continuous with respect to the weak operator topology, so they admit weakly continuous extensions to $M$. Clearly

$$
\delta(a)=W(a \otimes 1) W^{*}, \quad \tau_{t}(a)=Q^{2 i t} a Q^{-2 i t}
$$

for any element $a \in M$. By Theorem 3.8 the unitary antipode $R$ is normal (i.e. weakly continuous), so it admits a weakly continuous extension to an involutive antiautomorphism acting on $M$. If necessary, we shall use the notations $\delta^{M}, R^{M}$ and $\tau^{M}$ for them. The involutivity of the unitary antipode and the commutativity of the unitary antipode and the scaling group follow immediately.

The scaling group $\tau^{M}=\left\{\tau_{t}^{M}\right\}_{t \in \mathbb{R}}$ is no longer (pointwise) norm continuous so the theory presented in Appendix $\mathbf{F}$ can not be applied. Nevertheless one may speak about the analytic generator of this group in the weak sense: an element $a \in M$ belongs to the domain of $\tau_{i / 2}^{M}$ and $b=\tau_{i / 2}^{M}(a)$ if and only if for any $x, y \in \mathcal{H}$ there exists a continuous function $F_{x, y}$ defined on the strip $\{z \in \mathbb{C}: 0 \leq \Im z \leq 1 / 2\}$ holomorphic on the interior of this strip, such that $F_{x, y}(t)=\left(x \mid \tau_{t}^{M}(a) y\right)$ for all $t \in \mathbb{R}$ and $F_{x, y}(i / 2)=(x \mid b y)$. The antipode $\kappa^{M}$ on $M$ is defined by

$$
\kappa^{M}=\tau_{i / 2}^{M} R^{M} .
$$

In a sense $\tau_{i / 2}^{M}$ is a closure of $\tau_{i / 2}$. If $a \in \mathcal{D}\left(\tau_{i / 2}^{M}\right)$ and $b=\tau_{i / 2}^{M}(a)$ then there exists a net $\left\{a_{\varepsilon}\right\}_{\varepsilon>0}$ of elements of $\mathcal{D}\left(\tau_{i / 2}\right)$ converging to $a$ such that $\tau_{i / 2}\left(a_{\varepsilon}\right) \rightarrow b$ when $\varepsilon \rightarrow 0$ (both convergence in the sense of $\sigma$-weak topology). Indeed $a_{\varepsilon}=\frac{1}{\sqrt{\pi \varepsilon}} \int e^{-t^{2} / \varepsilon} \tau_{t}(a) d t$ does the job. In the same sense $\kappa^{M}$ is the closure of $\kappa$.

Let $\eta$ be the GNS-map associated with the right invariant weight $h$ on the $C^{*}$-algebra $A$. By the theory presented in Appendix $\mathbb{B}$ the double commutant $\eta^{\prime \prime}$ is an extension of $\eta$ and $\mathcal{D}(\eta)$ is a core for $\eta^{\prime \prime}$. It is a GNS-map defined on the von Neumann algebra $M$.

Using (2.1) one can easily show that $\mathcal{D}\left(\eta^{\prime \prime}\right)$ is $\tau^{M}$ - invariant and

$$
Q^{2 i t} \eta^{\prime \prime}(a)=\lambda^{-t / 2} \eta^{\prime \prime}\left(\tau_{t}^{M}(a)\right)
$$

for all $a \in \mathcal{D}\left(\eta^{\prime \prime}\right)$.

Proposition 4.1. Let $a \in \mathcal{D}\left(\eta^{\prime \prime}\right) \cap \mathcal{D}\left(\tau_{i / 2}^{M}\right)$ and $\eta^{\prime \prime}(a) \in \mathcal{D}\left(Q^{-1}\right)$. Then $\tau_{i / 2}^{M}(a) \in \mathcal{D}\left(\eta^{\prime \prime}\right)$ and

$$
\eta^{\prime \prime}\left(\tau_{i / 2}^{M}(a)\right)=\lambda^{i / 4} Q^{-1} \eta^{\prime \prime}(a) .
$$

Proof. Let $b \in \mathcal{D}\left(\eta^{\prime}\right)$ and $x \in \mathcal{H}$. Applying $b$ to the both sides of (4.1) and using (B.3), we obtain:

$$
b Q^{2 i t} \eta^{\prime \prime}(a)=\lambda^{-t / 2} \tau_{t}^{M}(a) \eta^{\prime}(b)
$$

and

$$
\left(x \mid b Q^{2 i t} \eta^{\prime \prime}(a)\right)=\lambda^{-t / 2}\left(x \mid \tau_{t}^{M}(a) \eta^{\prime}(b)\right)
$$

for any $t \in \mathbb{R}$. Remembering that $a \in \mathcal{D}\left(\tau_{i / 2}^{M}\right)$ and $\eta^{\prime \prime}(a) \in \mathcal{D}\left(Q^{-1}\right)$, we see that both sides of the above formula admit continuations to continuous functions on the strip $\{t \in \mathbb{C}: 0 \leq \Im t \leq 1 / 2\}$ holomorphic on the interior of this strip. Inserting $t=i / 2$ we obtain

$$
\left(x \mid b Q^{-1} \eta^{\prime \prime}(a)\right)=\lambda^{-i / 4}\left(x \mid \tau_{i / 2}^{M}(a) \eta^{\prime}(b)\right)
$$


and $b Q^{-1} \eta^{\prime \prime}(a)=\lambda^{-i / 4} \tau_{i / 2}^{M}(a) \eta^{\prime}(b)$. Therefore (cf. (B.3) $) \tau_{i / 2}^{M}(a) \in \mathcal{D}\left(\eta^{\prime \prime}\right)$ and (4.2) follows.

Let $\tau_{t}^{\prime}(b)=Q^{2 i t} b Q^{-2 i t}$ for $b \in A^{\prime}$. Since the scaling group $\left\{\tau_{t}\right\}_{t \in \mathbb{R}}$ on $A$ is implemented by $\left\{Q^{2 i t}\right\}_{t \in \mathbb{R}},\left\{\tau_{t}^{\prime}\right\}_{t \in \mathbb{R}}$ is a one parameter group of automorphisms of $A^{\prime}$. Let $b \in \mathcal{D}\left(\eta^{\prime}\right)$. Then for any $a \in \mathcal{D}(\eta)$ we have

$$
\tau_{t}^{\prime}(b) \eta(a)=\lambda^{t / 2} Q^{2 i t} b \eta\left(\tau_{-t}(a)\right)=\lambda^{t / 2} Q^{2 i t} \tau_{-t}(a) \eta^{\prime}(b)=\lambda^{t / 2} a Q^{2 i t} \eta^{\prime}(b) .
$$

Hence $\tau_{t}^{\prime}(b) \in \mathcal{D}\left(\eta^{\prime}\right)$ and

$$
\eta^{\prime}\left(\tau_{t}^{\prime}(b)\right)=\lambda^{t / 2} Q^{2 i t} \eta^{\prime}(b)
$$

Proposition 4.2. The set $\left\{c \in \mathcal{D}\left(\eta^{\prime}\right): \eta^{\prime}(c) \in \mathcal{D}\left(Q^{-1}\right) \cap \mathcal{D}(Q)\right\}$ is a core for $\eta^{\prime}$.

Proof. For $c \in A^{\prime}$ we put

$$
\mathcal{R}_{\varepsilon}(c)=\frac{1}{\sqrt{\pi \varepsilon}} \int_{\mathbb{R}} e^{-t^{2} / \varepsilon} \tau_{t}^{\prime}(c) d t .
$$

Let $c \in \mathcal{D}\left(\eta^{\prime}\right)$. The mapping $t \mapsto \tau_{t}^{\prime}(c)$ is strongly continuous and the mapping $t \mapsto \eta^{\prime}\left(\tau_{t}^{\prime}(c)\right)=$ $\lambda^{t / 2} Q^{2 i t} \eta^{\prime}(c)$ is norm continuous. Since $\eta^{\prime}$ is closed, we find that $\mathcal{R}_{\varepsilon}(c) \in \mathcal{D}\left(\eta^{\prime}\right)$ and

$$
\begin{aligned}
\eta^{\prime}\left(\mathcal{R}_{\varepsilon}(c)\right) & =\frac{1}{\sqrt{\pi \varepsilon}} \int_{\mathbb{R}} e^{-t^{2} / \varepsilon} \eta^{\prime}\left(\tau_{t}^{\prime}(c)\right) d t \\
& =\frac{1}{\sqrt{\pi \varepsilon}} \int_{\mathbb{R}} \lambda^{t / 2} e^{-t^{2} / \varepsilon} Q^{2 i t} d t \eta^{\prime}(c)=e^{-\varepsilon(\log Q-i \log \lambda / 4)^{2}} \eta^{\prime}(c) .
\end{aligned}
$$

Using this formula one can easily verify that $\eta^{\prime}\left(\mathcal{R}_{\varepsilon}(c)\right) \in \mathcal{D}\left(Q^{-1}\right) \cap \mathcal{D}(Q)$. Clearly $\eta^{\prime}\left(\mathcal{R}_{\varepsilon}(c)\right)$ converges in norm to $\eta^{\prime}(c)$ as $\varepsilon$ tends to 0 . One can also easily verify that $\mathcal{R}_{\varepsilon}(c)$ converges strongly to $c$. Therefore the set $\left\{c \in \mathcal{D}\left(\eta^{\prime}\right): \eta^{\prime}(c) \in \mathcal{D}\left(Q^{-1}\right) \cap \mathcal{D}(Q)\right\}$ is a core for $\eta^{\prime}$.

One can easily verify that the right hand sides of (1.1) make sense for $a \in M$ and $\varphi, \psi \in A_{*}$. In other words the convolution products $\varphi * a$ and $a * \psi$ (with $\varphi, \psi \in A_{*}$ ) defined originally for $a \in A$ admit strongly continuous extension to $a \in M$.

Using (1.6) and remembering that $\mathcal{D}(\eta)$ is a core for $\eta^{\prime \prime}$, we see that $\varphi * a \in \mathcal{D}\left(\eta^{\prime \prime}\right)$ and

$$
W_{\varphi} \eta^{\prime \prime}(a)=\eta^{\prime \prime}(\varphi * a)
$$

for any $a \in \mathcal{D}\left(\eta^{\prime \prime}\right)$ and $\varphi \in A_{*}$.

In what follows, we denote by the same letter $h$ the natural extension of the right Haar weight to $M$. By definition $h$ is the semifinite normal weight on $M$ related to the GNS map $\eta^{\prime \prime}$ via formula (C.8). By the strict faithfulness of the Haar weight, $h$ is faithful. Remembering that $R$ is normal we conclude that $h^{L}:=h \circ R$ is also a faithful semifinite normal weight on $M$. This is the left Haar weight. Let $\left\{\sigma_{t}\right\}_{t \in \mathbb{R}}$ and $\left\{\sigma_{t}^{L}\right\}_{t \in \mathbb{R}}$ be the modular automorphism groups of $M$ associated with $h$ and $h^{L}$.

Lemma 4.3. The following four formulae hold on the von Neumann algebra $M$.

(1) $R \circ \sigma_{t}^{o} R=\sigma_{-t}^{L}$ for $t \in \mathbb{R}$.

(2) $\delta \sigma^{\circ} \sigma_{t}=\left(\sigma_{t} \otimes \tau_{t}\right) \circ \delta$ for $t \in \mathbb{R}$.

(3) $\delta \sigma_{s}^{L}=\left(\tau_{-s} \otimes \sigma_{s}^{L}\right)$ o $\delta$ for $s \in \mathbb{R}$.

(4) $\sigma_{t^{o} \sigma_{s}^{L}}^{L}=\sigma_{s}^{L} \sigma_{t}$ for $t, s \in \mathbb{R}$.

Proof.

Ad 1. Follows immediately from Proposition D.8

Ad 2. Using (3.10) we compute:

$$
\begin{aligned}
\delta\left(\sigma_{t}(a)\right) & =\operatorname{Ad}_{W^{\circ}} \operatorname{Ad}_{\Delta^{i t} \otimes Q^{2 i t}}(a \otimes 1) \\
& =\operatorname{Ad}_{\Delta^{i t} \otimes Q^{2 i t} \circ \operatorname{Ad}_{W}}(a \otimes 1) \\
& =\left(\sigma_{t} \otimes \tau_{t}\right) \circ \delta(a) .
\end{aligned}
$$

Ad 3. We know (cf. (2.3) ) that $Q$ and $\Delta$ strongly commute. Therefore scaling automorphisms $\tau_{t}$ commute with modular automorphisms $\sigma_{s}$. Furthermore $\tau_{t}$ commute with $R$. Using Assertion 
1 we see that $\tau_{t}$ commute with $\sigma_{s}^{L}$. The rest is a matter of computation. Denoting by $\sigma$ the flip automorphism acting on $A \otimes A$ and using twice the first formula of Proposition [2.6] we obtain

$$
\begin{aligned}
\delta \circ \sigma_{t}^{L} & =\delta \circ R \circ \sigma_{-t} \circ R \\
& =\sigma \circ(R \otimes R) \circ \delta \circ \sigma_{-t} \circ R \\
& =\sigma \circ\left(\left(R \circ \sigma_{-t}\right) \otimes\left(R \circ \tau_{-t}\right)\right) \circ \delta \circ R \\
& =\sigma \circ\left(\left(R \circ \sigma_{-t} \circ R\right) \otimes\left(R \circ \tau_{-t} \circ R\right)\right) \circ \sigma \circ \delta \\
& =\left(\tau_{-t} \otimes \sigma_{t}^{L}\right) \circ \delta .
\end{aligned}
$$

Ad 4. We already know that the scaling automorphisms commute with the modular automorphisms. Therefore $\left(\tau_{-t} \otimes \sigma_{t}^{L}\right) \circ\left(\sigma_{s} \otimes \tau_{s}\right)=\left(\sigma_{s} \otimes \tau_{s}\right) \circ\left(\tau_{-t} \otimes \sigma_{t}^{L}\right)$ and

$$
\begin{aligned}
\delta \circ \sigma_{t}^{L} \circ \sigma_{s} & =\left(\tau_{-t} \otimes \sigma_{t}^{L}\right) \circ \delta \circ \sigma_{s}=\left(\tau_{-t} \otimes \sigma_{t}^{L}\right) \circ\left(\sigma_{s} \otimes \tau_{s}\right) \circ \delta \\
& =\left(\sigma_{s} \otimes \tau_{s}\right) \circ\left(\tau_{-t} \otimes \sigma_{t}^{L}\right) \circ \delta=\left(\sigma_{s} \otimes \tau_{s}\right) \circ \delta \sigma_{t}^{L}=\delta \circ \sigma_{s} \circ \sigma_{t}^{L} .
\end{aligned}
$$

Now, using the injectivity of the comultiplication we obtain $\sigma_{t}^{L} \circ \sigma_{s}=\sigma_{s} \circ \sigma_{t}^{L}$.

Corollary 4.4. If $h$ is a trace then $\kappa$ coincides with the unitary antipode $R$.

Proof. Let $t \in \mathbb{R}$. If $h$ is a trace then $\sigma_{t}=\mathrm{id}$ and by Statement $2, \mathrm{id} \otimes \tau_{t}$ coincides with the identity on $\delta(A)$. It follows that id $\otimes \tau_{t}$ coincides with the identity on $(A \otimes 1) \delta(A)$. By the cancellation property the latter set is linearly dense in $A \otimes A$. Hence $\tau_{t}=$ id for all $t \in \mathbb{R}$. Formula (1.3) shows now that $\kappa=R$.

We now consider the relative modular operator determined by $h$ and $h^{L}=h \circ R$. Let $h^{\prime}$ be the

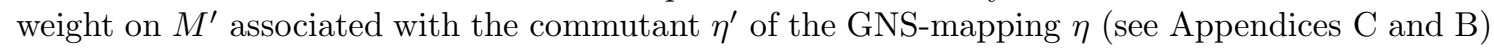
and let $\Delta_{\text {rel }}$ denote the spatial derivative $d h^{L} / d h^{\prime}$ in the sense of Connes [3] (see Appendix [D]). Then $\Delta_{\text {rel }}$ is a strictly positive self-adjoint operator with a core $\left\{\eta^{\prime \prime}(a): a \in \mathcal{D}\left(\eta^{\prime \prime}\right), h^{L}\left(a a^{*}\right)<\infty\right\}$, such that

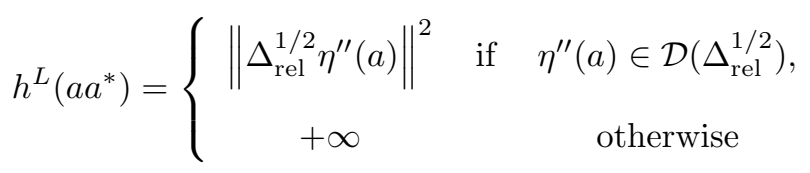

for any $a \in \mathcal{D}\left(\eta^{\prime \prime}\right)$. It is known that the modular automorphism group $\sigma^{L}$ is implemented by $\Delta_{\text {rel }}$ :

$$
\sigma_{t}^{L}(a)=\Delta_{\text {rel }}^{i t} a \Delta_{\text {rel }}^{-i t}
$$

for any $a \in M$ and $t \in \mathbb{R}$. Moreover the Connes' Radon-Nikodym cocycle $\left(D h^{L}: D h\right)_{t}$ is expressed by the formula

$$
\left(D h^{L}: D h\right)_{t}=\Delta_{\text {rel }}^{i t} \Delta^{-i t} \in M .
$$

Proposition 4.5. There exist a strictly positive self-adjoint operator $\gamma$ affiliated with the center of the von Neumann algebra $M$ and a strictly positive self-adjoint operator $\rho$ affiliated with the von Neumann algebra $M$ such that

$$
\left(D h^{L}: D h\right)_{t}=\gamma^{i t^{2} / 2} \rho^{i t}
$$

for all $t \in \mathbb{R}$. Operators $\rho$ and $\gamma$ are uniquely determined by the above formula. They satisfy the following commutation relations:

$$
\begin{array}{ll}
\Delta^{i t} \rho \Delta^{-i t}=\gamma^{t} \rho, & \Delta^{i t} \gamma \Delta^{-i t}=\gamma \\
Q^{i t} \rho Q^{-i t}=\rho, & Q^{i t} \gamma Q^{-i t}=\gamma .
\end{array}
$$

for all $t \in \mathbb{R}$.

Proof. For any $t, s \in \mathbb{R}$, we set

$$
\gamma(t, s):=\Delta^{i t} \Delta_{\mathrm{rel}}^{i s} \Delta^{-i t} \Delta_{\mathrm{rel}}^{-i s} .
$$

Clearly $\left.\operatorname{Ad}_{\gamma(t, s)}\right|_{M}=\sigma_{t} \sigma_{s}^{L} \sigma_{-t} \sigma_{-s}^{L}$. By Assertion 4 of Lemma 4.3 the latter automorphism coincides with the identity on $M$. Therefore $\gamma(t, s)$ commutes with all elements of $M: \gamma(t, s) \in M^{\prime}$. On the other hand one can easily verify that

$$
\gamma(t, s)=\operatorname{Ad}_{\Delta^{i t}}\left(\Delta_{\mathrm{rel}}^{i s} \Delta^{-i s}\right)\left(\Delta_{\mathrm{rel}}^{i s} \Delta^{-i s}\right)^{*}=\sigma_{t}\left(\left(D h^{L}: D h\right)_{s}\right)\left(D h^{L}: D h\right)_{s}^{*} .
$$


Therefore $\gamma(t, s) \in M$. This way we showed that the unitary $\gamma(t, s)$ belongs to the center of $M$. It is known that central elements are invariant under the action of modular automorphisms. Therefore $\gamma(t, s)$ commutes with $\Delta^{i t}$ and $\Delta_{\text {rel }}^{i s}$.

Rewriting now (4.9) in the form $\gamma(t, s) \Delta_{\text {rel }}^{i s}=\Delta^{i t} \Delta_{\text {rel }}^{i s} \Delta^{-i t}$ one can easily show that $\gamma\left(t, s+s^{\prime}\right)=$ $\gamma(t, s) \gamma\left(t, s^{\prime}\right)$ for all $t, s, s^{\prime} \in \mathbb{R}$. Similarly using the formula $\Delta^{-i t} \gamma(t, s)=\Delta_{\text {rel }}^{i s} \Delta^{-i t} \Delta_{\text {rel }}^{-i s}$ one can easily show that $\gamma\left(t+t^{\prime}, s\right)=\gamma(t, s) \gamma\left(t^{\prime}, s\right)$ for all $t, t^{\prime}, s \in \mathbb{R}$.

By the above results $\gamma(t, s)$ is of the form

$$
\gamma(t, s)=\gamma^{i s t}
$$

where $\gamma$ is a strictly positive self-adjoint operator affiliated with the center of $M$. Clearly $\gamma$ strongly commutes with $\Delta$ and $\Delta_{\text {rel }}$. Therefore the second formula of (4.7) holds.

Let $t, s \in \mathbb{R}$. Then (4.9) shows that $\Delta^{-i t} \Delta_{\text {rel }}^{i s}=\gamma^{-i t s} \Delta_{\text {rel }}^{i s} \Delta^{-i t}$. Therefore

$$
\begin{aligned}
\gamma^{-i t^{2} / 2} \Delta_{\text {rel }}^{i t} \Delta^{-i t} \gamma^{-i s^{2} / 2} \Delta_{\text {rel }}^{i s} \Delta^{-i s} & =\gamma^{-i t^{2} / 2-i t s-i s^{2} / 2} \Delta_{\text {rel }}^{i t+i s} \Delta^{-i t-i s} \\
& =\gamma^{-i(t+s)^{2} / 2} \Delta_{\text {rel }}^{i(t+s)} \Delta^{-i(t+s)} .
\end{aligned}
$$

It shows that $\left\{\gamma^{-i t^{2} / 2} \Delta_{\text {rel }}^{i t} \Delta^{-i t}\right\}_{t \in \mathbb{R}}$ is a one parameter group of unitaries. By (4.5) these unitaries belong to $M$. Therefore there exists a strictly positive self-adjoint operator $\rho$ affiliated with the von Neumann algebra $M$ such that

$$
\rho^{i t}=\gamma^{-i t^{2} / 2} \Delta_{\text {rel }}^{i t} \Delta^{-i t}
$$

for all $t \in \mathbb{R}$. Clearly (4.6) is equivalent to the above formula.

Inserting $\frac{t}{n}$ instead of $t$ and taking the $n$-th power of the both sides we get

$$
\rho^{i t}=\gamma^{i t^{2} / 2 n}\left(\Delta_{\text {rel }}^{i t / n} \Delta^{-i t / n}\right)^{n} .
$$

Letting $n \rightarrow \infty$ we obtain

$$
\rho^{i t}=\operatorname{s-lim}_{n \rightarrow \infty}\left(\Delta_{\text {rel }}^{i t / n} \Delta^{-i t / n}\right)^{n} .
$$

This formula shows the uniqueness of $\rho$ (and $\gamma$ ).

To prove (4.8) we notice that $\tau_{t}$ scales $h$ and $h^{L}$ by the same factor $\lambda^{t}$ (this is because $\tau_{t}$ and $R$ commute). Therefore the Connes' Radon-Nikodym cocycle (4.5) is $\tau_{t}$ invariant. Using the uniqueness of $\rho$ and $\gamma$ we obtain: $\tau_{t}(\rho)=\rho, \tau_{t}(\gamma)=\gamma$ and (4.8) follows.

By (4.6), $\Delta_{\text {rel }}^{i t}=\gamma^{i t^{2} / 2} \rho^{i t} \Delta^{i t}$. Therefore for any $t, s \in \mathbb{R}$ we have:

$$
\gamma^{i(t+s)^{2} / 2} \rho^{i(t+s)} \Delta^{i(t+s)}=\gamma^{i t^{2} / 2} \rho^{i t} \Delta^{i t} \gamma^{i s^{2} / 2} \rho^{i s} \Delta^{i s} .
$$

By simple computation this formula reduces to

$$
\gamma^{i t s} \rho^{i s} \Delta^{i t}=\Delta^{i t} \rho^{i s} .
$$

Therefore $\left(\gamma^{t} \rho\right)^{i s}=\Delta^{i t} \rho^{i s} \Delta^{-i t}$ and the first formula of (4.7) follows.

Formulae (4.7) and (4.8) show that

$$
\begin{array}{ll}
\sigma_{t}(\rho)=\gamma^{t} \rho, & \sigma_{t}(\gamma)=\gamma \\
\tau_{t}(\rho)=\rho, & \tau_{t}(\gamma)=\gamma .
\end{array}
$$

for all $t \in \mathbb{R}$.

Proposition 4.6. The operators $\rho$ and $\gamma$ satisfy $R\left(\rho^{i t}\right)=\rho^{-i t}$ and $R\left(\gamma^{i t}\right)=\gamma^{i t}$ for $t \in \mathbb{R}$. In other words $R(\rho)=\rho^{-1}$ and $R(\gamma)=\gamma$.

Proof. Inserting $h_{1}=h^{L}$ in Assertion 2 of Proposition D.8 we obtain

$$
\left(D h^{L}: D h\right)_{t}=R\left(\left(D h^{L}: D h\right)_{-t}\right)=R\left(\gamma^{i t^{2} / 2} \rho^{-i t}\right)=R(\gamma)^{i t^{2} / 2} R(\rho)^{-i t} .
$$

Using the uniqueness of $\rho$ and $\gamma$ we get: $R(\rho)=\rho^{-1}$ and $R(\gamma)=\gamma$. 
Formula (4.11) shows that one parameter groups $\left\{\rho^{i t}\right\}_{t \in \mathbb{R}},\left\{\Delta^{i t}\right\}_{t \in \mathbb{R}}$ and $\left\{\gamma^{i t}\right\}_{t \in \mathbb{R}}$ generate a unitary representation of the Heisenberg group. Using the formula $\Delta_{\text {rel }}^{i t}=\gamma^{i t^{2} / 2} \rho^{i t} \Delta^{i t}$ we see that $\left\{\Delta_{\text {rel }}^{i t}\right\}_{t \in \mathbb{R}}$ is an another one parameter group appearing in this representation. Its infinitesimal generator $\log \Delta_{\text {rel }}=\log \Delta+\log \rho$. Then (4.10) is the Trotter product formula corresponding to the relation $\log \rho=\log \Delta_{\text {rel }}-\log \Delta$. (cf. [25]).

Inserting $s=t$ in (4.11) we obtain $\gamma^{i t^{2}} \rho^{i t} \Delta^{i t}=\Delta^{i t} \rho^{i t}$. Combining (4.6) with (4.5) we get

$$
\Delta_{\text {rel }}^{i t}=\gamma^{i t^{2} / 2} \rho^{i t} \Delta^{i t}=\gamma^{-i t^{2} / 2} \Delta^{i t} \rho^{i t}
$$

for $t \in \mathbb{R}$. Now we put $t:=-i / 2$ to obtain

$$
\Delta_{\mathrm{rel}}^{1 / 2}=\gamma^{-i / 8} \overline{\rho^{1 / 2} \circ \Delta^{1 / 2}}=\gamma^{i / 8} \overline{\Delta^{1 / 2} \circ \rho^{1 / 2}} .
$$

In particular $\mathcal{D}\left(\rho^{1 / 2} \circ \Delta^{1 / 2}\right) \subset \mathcal{D}\left(\Delta_{\text {rel }}^{1 / 2}\right)$. In these formulae $\bar{X}$ denotes the closure of an operator $X$ and $X \circ Y$ denotes the composition of operators $X$ and $Y$. By definition $\mathcal{D}(X \circ Y)=$ $\{x \in \mathcal{D}(Y): Y x \in \mathcal{D}(X)\}$.

The following result plays an important role in the paper.

\section{Proposition 4.7.}

1. If $a \in M$ and $\delta(a)=1 \otimes a$ then $a \in \mathbb{C} 1$.

2. If $a \in M$ and $\delta(a)=a \otimes 1$ then $a \in \mathbb{C} 1$.

3. $M \cap \widehat{A}^{\prime}=\mathbb{C} 1$.

Proof.

Ad 1. By Proposition 2.6 $\delta$ commutes with the scaling group. Therefore the set

$$
\{a \in M: \delta(a)=1 \otimes a\}
$$

is $\tau$ invariant and we may assume that $a$ is an entire analytical element for $\tau$. Using Statement 2 of Lemma 4.3 we have: $\delta\left(\sigma_{t}(a)\right)=\left(\sigma_{t} \otimes \tau_{t}\right)(1 \otimes a)=1 \otimes \tau_{t}(a)$. It shows that $a$ is an entire analytical element for the modular automorphism group. Hence (see Theorem D.7), for any $b \in \mathcal{D}(\eta)$ we have $b a \in \mathcal{D}\left(\eta^{\prime \prime}\right)$. Therefore for any $x \in \mathcal{H}$ we have

$$
\begin{aligned}
W\left(\eta^{\prime \prime}(b a) \otimes x\right) & =\sum_{n=1}^{\infty} \delta(b a)\left(\Omega_{n} \otimes x\right)=\sum_{n=1}^{\infty} \delta(b)\left(\Omega_{n} \otimes a x\right) \\
& =W(\eta(b) \otimes a x) .
\end{aligned}
$$

Hence $\eta^{\prime \prime}(b a) \otimes x=\eta(b) \otimes a x$ and for any $c \in \mathcal{D}\left(\eta^{\prime}\right)$ we have:

$$
b a \eta^{\prime}(c) \otimes x=c \eta^{\prime \prime}(b a) \otimes x=c \eta(b) \otimes a x=b \eta^{\prime}(c) \otimes a x .
$$

It shows that $a \otimes 1=1 \otimes a$ and $a$ must be a scalar multiples of the identity.

Ad 2. Using the first Statement of Proposition 2.6 we have:

$$
\delta\left(R^{M}(a)\right)=\sigma \circ\left(R^{M} \otimes R^{M}\right) \delta(a)=\sigma \circ\left(R^{M} \otimes R^{M}\right)(a \otimes 1)=1 \otimes R^{M}(a) .
$$

Therefore, by Statement $1, R^{M}(a) \in \mathbb{C} 1$ and finally $a \in \mathbb{C} 1$.

Ad 3. We know that $W \in M(\widehat{A} \otimes A)$. Formula (3.7) shows that $\delta(a)=a \otimes 1$ for any $a \in M \cap \widehat{A}^{\prime}$ and Statement 3 follows immediately from Statement 2.

Let $L S$ ('LS' stays for left shifts) be the set of all automorphisms $\alpha$ of the von Neumann algebra $M$ such that

$$
\delta \circ \alpha=(\alpha \otimes \mathrm{id}) \circ \delta .
$$

We shall use the $u$-topology (cf. [23, Section 2.23]) on the set of automorphisms of $M$. By definition a net $\alpha_{n}$ is $u$-convergent to $\alpha_{\infty}$ if for any $\varphi \in M_{*}$, the net of functionals $\varphi^{\circ} \alpha_{n}$ converges in norm to $\varphi^{\circ} \alpha_{\infty}$. One can easily verify that $L S$ endowed with the $u$-topology is a topological group. In particular $\alpha_{n}^{-1} \rightarrow \alpha_{\infty}^{-1}$ if $\alpha_{n} \rightarrow \alpha_{\infty}$

Let $t \in \mathbb{R}$. Combining Statement 2 of Proposition 2.6 with Statement 2 of Lemma 4.3 one can easily verify that $\delta \circ \sigma_{t} \circ \tau_{-t}=\left(\sigma_{t} \circ \tau_{-t} \otimes \mathrm{id}\right) \circ \delta$. It shows that

$$
\sigma_{t^{\circ}} \tau_{-t} \in L S
$$

It is easy to see that the mapping $\mathbb{R} \ni t \mapsto \sigma_{t^{\circ}} \tau_{-t}$ is $u$-continuous. 
Proposition 4.8. Let $a \in A$. Then $\alpha(a) \in A$ for any $\alpha \in L S$. Moreover the mapping

$$
L S \ni \alpha \longmapsto \alpha(a) \in A
$$

is norm continuous (we use u-topology on $L S$ ).

Proof. It is sufficient to prove this statement for $a$ running over a linearly dense subset of $A$. Let $\alpha \in L S$. Using (3.8) and (4.16), one can easily verify that

$$
(\mathrm{id} \otimes \delta)\left((\mathrm{id} \otimes \alpha)(W) W^{*}\right)=\left((\mathrm{id} \otimes \alpha)(W) W^{*}\right) \otimes 1 .
$$

Indeed

$$
\begin{aligned}
(\mathrm{id} \otimes \delta)\left((\mathrm{id} \otimes \alpha)(W) W^{*}\right) & =(\mathrm{id} \otimes \delta \circ \alpha) W(\mathrm{id} \otimes \delta) W^{*}=(\mathrm{id} \otimes \alpha \otimes \mathrm{id})(\mathrm{id} \otimes \delta) W(\mathrm{id} \otimes \delta) W^{*} \\
& =\left((\mathrm{id} \otimes \alpha \otimes \mathrm{id}) W_{12} W_{13}\right)\left(W_{12} W_{13}\right)^{*}=\left((\mathrm{id} \otimes \alpha)(W) W^{*}\right)_{12}
\end{aligned}
$$

and (4.18) follows. Using now Statement 2 of Proposition 4.7 we conclude that (id $\otimes \alpha)(W) W^{*}$ is of the form $v \otimes 1$, where $v \in \mathcal{L}(\mathcal{H})$. Hence

$$
(\text { id } \otimes \alpha)(W)=(v \otimes 1) W .
$$

Clearly $v$ is unitary. Passing from $\mathcal{H} \otimes \mathcal{H}$ to $\mathcal{H} \otimes \mathcal{H} \otimes \mathcal{H}$ we obtain:

$$
\begin{aligned}
& (\mathrm{id} \otimes \alpha \otimes \mathrm{id})\left(W_{12}\right)=(v \otimes 1 \otimes 1) W_{12}, \\
& (\mathrm{id} \otimes \mathrm{id} \otimes \alpha)\left(W_{13}\right)=(v \otimes 1 \otimes 1) W_{13} .
\end{aligned}
$$

Eliminating $(v \otimes 1 \otimes 1)$ we get:

$$
(\mathrm{id} \otimes \alpha \otimes \alpha)\left(W_{12}^{*} W_{13}\right)=W_{12}^{*} W_{13}
$$

and finally

$$
(\mathrm{id} \otimes \mathrm{id} \otimes \alpha)\left(W_{12}^{*} W_{13}\right)=\left(\mathrm{id} \otimes \alpha^{-1} \otimes \mathrm{id}\right)\left(W_{12}^{*} W_{13}\right) .
$$

Let $x, y \in \mathcal{H}$ and $\mu \in M_{*}$. Applying $\omega_{x, y} \otimes \mu^{*} \otimes$ id to the both sides of (4.19) we obtain:

$$
\alpha\left(W\left(W_{\mu} x, y\right)\right)=W\left(W_{\mu^{\circ} \alpha^{-1}} x, y\right) .
$$

The reader should notice that the right hand side of the above formula belongs to $A$ and depends continuously on $\alpha$. This way our statement is proved for all $a$ of the form $a=W\left(W_{\mu} x, y\right)$. To end the proof we recall that $\widehat{A}$ act on $\mathcal{H}$ in a nondegenerate way. Therefore the set of vectors $\left\{W_{\mu} x: \mu \in \mathcal{L}(\mathcal{H})_{*}, x \in \mathcal{H}\right\}$ is dense in $\mathcal{H}$. Proposition 1.12 shows now that the set

$$
\left\{W\left(W_{\mu} x, y\right): \mu \in \mathcal{L}(\mathcal{H})_{*} ; x, y \in \mathcal{H}\right\}
$$

is linearly dense in $A$.

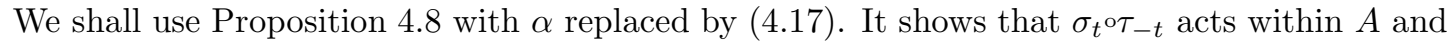
that for any $a \in A, \sigma_{t^{\circ} \tau_{-t}}(a)$ depends on $t \in \mathbb{R}$ in a norm continuous way. Combining this result with the known properties of the scaling group, we obtain

Proposition 4.9. The modular automorphism group $\left\{\sigma_{t}\right\}_{t \in \mathbb{R}}$ of $M$ associated with the Haar weight $h$ may be restricted to A. It defines the pointwise norm continuous one parameter group of automorphisms of $A$.

In this way we have shown Assertion 3 of Theorem 1.9 Restricting the second formula of Lemma 4.3 to $A$, we obtain Assertion 4 of Theorem 1.9

The end of this Section is devoted to the proof of the uniqueness of the antipode and the Haar weight.

Theorem 4.10. Let $(A, \delta)$ be a weighted Hopf $C^{*}$-algebra with the Haar weight $h$ and antipode $\kappa$. Assume that we have another antipode $\kappa_{1}$ with corresponding Haar weight $h_{1}$ satisfying all the requirements of Definition 1.5. Then $\kappa_{1}=\kappa$ and $h_{1}=\mu h$ for some positive scalar $\mu$.

Proof. Let $\left(\mathcal{H}_{1}, \pi_{1}, \eta_{1}\right)$ be the GNS triple related to $h_{1}$. Then we have another Kac-Takesaki operator $W_{1} \in \mathcal{L}\left(\mathcal{H}_{1} \otimes \mathcal{H}_{1}\right)$ that gives rise to $(A, \delta)$. By a Theorem in 35 we may assume that $\mathcal{H}_{1}=\mathcal{H}$ and $\pi_{1}=\pi$. Combining the formula $(\mathrm{id} \otimes \delta)\left(W_{1}\right)=\left(W_{1}\right)_{12}\left(W_{1}\right)_{13}$ with 3.7) we obtain: $W_{23}\left(W_{1}\right)_{12} W_{23}^{*}=\left(W_{1}\right)_{12}\left(W_{1}\right)_{13}$. It means that $W_{1}$ is adapted to $W$ in the sense of 34. Definition 1.3]. Statement 4 of Theorem 1.6 of the same reference shows now that $\kappa_{1} \subset \kappa$. Interchanging the roles of $W$ and $W_{1}$ we obtain the converse inclusion. Therefore

$$
\kappa_{1}=\kappa .
$$


We shall use the relative conjugate linear Tomita-Takesaki operators $\widetilde{S}=\widetilde{F}^{*}$. By definition the set $\left\{\eta(a): a \in \mathcal{D}(\eta) \cap \mathcal{D}\left(\eta_{1}\right)^{*}\right\}$ is a core for $\widetilde{S}$ and

$$
\widetilde{S} \eta(a)=\eta_{1}\left(a^{*}\right)
$$

for any $a \in \mathcal{D}(\eta) \cap \mathcal{D}\left(\eta_{1}\right)^{*}$. For any $a \in \mathcal{D}(\eta) \cap \mathcal{D}\left(\eta_{1}\right)^{*}$ and $\varphi \in A^{*}$ we have $\varphi^{*} * a \in \mathcal{D}(\eta)$ and $\varphi * a^{*} \in \mathcal{D}\left(\eta_{1}\right)$. Therefore for any $z \in \mathcal{D}(\widetilde{F})$ we have

$$
\begin{aligned}
& \varphi\left(W_{1}\left(z, \eta_{1}\left(a^{*}\right)\right)\right)=\left(z \mid\left(W_{1}\right)_{\varphi} \eta_{1}\left(a^{*}\right)\right)=\left(z \mid \eta_{1}\left(\varphi * a^{*}\right)\right) \\
& =\left(z \mid \widetilde{S} \eta\left(\varphi^{*} * a\right)\right)=\overline{\left(\widetilde{F} z \mid W_{\varphi^{*}} \eta(a)\right)} \\
& =\overline{\varphi^{*}(W(\widetilde{F} z, \eta(a)))}=\varphi\left(W(\widetilde{F} z, \eta(a))^{*}\right) .
\end{aligned}
$$

Hence we have $W_{1}\left(z, \eta_{1}\left(a^{*}\right)\right)=W(\widetilde{F} z, \eta(a))^{*}$. Remembering that $\left\{\eta(a): a \in \mathcal{D}(\eta) \cap \mathcal{D}\left(\eta_{1}\right)^{*}\right\}$ is a core for $\widetilde{S}$, we conclude that $W_{1}(z, \widetilde{S} x)=W(\widetilde{F} z, x)^{*}$ for any $x \in \mathcal{D}(\widetilde{S})$. Setting $y=\widetilde{F} z$ we get

$$
W_{1}\left(\widetilde{F}^{-1} y, \widetilde{S} x\right)=W(y, x)^{*} .
$$

This formula holds for any $x \in \mathcal{D}(\widetilde{S})$ and $y \in \mathcal{D}\left(\widetilde{F}^{-1}\right)$.

Let $\widetilde{S}=\widetilde{J} \widetilde{\Delta}^{1 / 2}$ be the polar decomposition of $\widetilde{S}$. Then $\widetilde{F}=\widetilde{\Delta}^{1 / 2} \widetilde{J}^{-1}$. For any $x \in \mathcal{D}(\widetilde{\Delta})$ and $y \in \mathcal{D}\left(\widetilde{\Delta}^{-1}\right)$, we have $\widetilde{S} x \in \mathcal{D}(\widetilde{F})$ and $\widetilde{F}^{-1} y \in \mathcal{D}\left(\widetilde{S}^{-1}\right)$. Using (4.22) and (4.20) and Proposition 2.4 we get

$$
\begin{aligned}
\kappa^{2}(W(x, y)) & =\kappa\left(W(y, x)^{*}\right)=\kappa\left(W_{1}\left(\widetilde{F}^{-1} y, \widetilde{S} x\right)\right)=\kappa_{1}\left(W_{1}\left(\widetilde{F}^{-1} y, \widetilde{S} x\right)\right) \\
& =W_{1}\left(\widetilde{S} x, \widetilde{F}^{-1} y\right)^{*}=W\left(\widetilde{F} \widetilde{S} x, \widetilde{S}^{-1} \widetilde{F}^{-1} y\right)=W\left(\widetilde{\Delta} x, \widetilde{\Delta}^{-1} y\right) .
\end{aligned}
$$

Remembering that $\kappa^{2}=\tau_{i}$ and using Theorem F.3, we obtain

$$
\tau_{t}(W(x, y))=W\left(\widetilde{\Delta}^{i t} x, \widetilde{\Delta}^{i t} y\right) .
$$

for all $t \in \mathbb{R}$. On the other hand, $\tau_{t}(W(x, y))=W\left(\Delta^{i t} x, \Delta^{i t} y\right)$ by Proposition 2.2 Therefore $W\left(\widetilde{\Delta}^{i t} x, \widetilde{\Delta}^{i t} y\right)=W\left(\Delta^{i t} x, \Delta^{i t} y\right)$ and $\widetilde{\Delta}^{-i t} \Delta^{i t}$ commutes with $W_{\varphi}$ for all $\varphi \in A^{*}$. Hence it belongs to the commutant of $\widehat{A}$. On the other hand the Radon-Nikodym cocycle $\left(D h_{1}: D h\right)_{-t}=\widetilde{\Delta}^{-i t} \Delta^{i t} \in$ $M$. Therefore by Proposition 4.7 it must be a scalar multiple of the identity and there exists a positive scalar $\mu$ such that $\widetilde{\Delta}^{i t}=\mu^{i t} \Delta^{i t}$ for all $t \in \mathbb{R}$. Hence $\widetilde{\Delta}=\mu \Delta$. In particular $\mathcal{D}(\widetilde{S})=$ $\mathcal{D}\left(\widetilde{\Delta}^{1 / 2}\right)=\mathcal{D}\left(\Delta^{1 / 2}\right)$.

The reader should notice that $\mathcal{D}(\eta)^{*}$ is a right ideal and $\mathcal{D}\left(\eta_{1}\right)$ is a left ideal in $A$. Therefore $\mathcal{D}(\eta)^{*} \mathcal{D}\left(\eta_{1}\right) \subset \mathcal{D}(\eta)^{*} \cap \mathcal{D}\left(\eta_{1}\right)$. Using the norm density of $\mathcal{D}(\eta)^{*}$ one can easily show that $\mathcal{D}(\eta)^{*} \mathcal{D}\left(\eta_{1}\right)$ is a core for $\eta_{1}$. So is $\mathcal{D}(\eta)^{*} \cap \mathcal{D}\left(\eta_{1}\right)$. Let $a \in \mathcal{D}(\eta)^{*} \cap \mathcal{D}\left(\eta_{1}\right)$. Then (cf. 44.21) $) \eta\left(a^{*}\right) \in \mathcal{D}(\widetilde{S})=$ $\mathcal{D}\left(\Delta^{1 / 2}\right)$ and using Theorem D.5 we see that $a \in \mathcal{D}(\eta)$. Moreover

$$
\eta_{1}(a)=\widetilde{J} \widetilde{\Delta}^{1 / 2} \eta\left(a^{*}\right)=\sqrt{\mu} \widetilde{J} \Delta^{1 / 2} \eta\left(a^{*}\right)=\sqrt{\mu} \widetilde{J} J \eta(a) .
$$

Remembering that $\mathcal{D}(\eta)^{*} \cap \mathcal{D}\left(\eta_{1}\right)$ is a core for $\eta_{1}$ we see that $\mathcal{D}\left(\eta_{1}\right) \subset \mathcal{D}(\eta)$ and that the above formula holds for all $a \in \mathcal{D}\left(\eta_{1}\right)$. Interchanging the roles of $\eta$ and $\eta_{1}$ we obtain the converse inclusion. Hence $\mathcal{D}\left(\eta_{1}\right)=\mathcal{D}(\eta)$. Formula (4.23) shows now that $h_{1}\left(a^{*} a\right)=\mu h\left(a^{*} a\right)$ for any $a \in A$.

\section{Modular Structure for the Dual}

We now go into the discussion on the density theorem. Combining (2.4) and (4.8), we know that $J \rho^{-1} J$ and $Q$ are strongly commuting strictly positive self-adjoint operators. Therefore the operator

$$
\widehat{\Delta}:=\overline{J \rho^{-1} J \circ Q^{2}}
$$

is also strictly positive and self-adjoint. This section is devoted to the proof of the following theorem. It contains the inverse of the square root of (5.1).

Theorem 5.1. Let

$$
\mathcal{D}_{0}=\left\{b \in \mathcal{D}\left(\eta^{\prime \prime}\right) \cap \mathcal{D}\left(\kappa^{M}\right): \kappa^{M}(b)^{*} \in \mathcal{D}\left(\eta^{\prime \prime}\right)\right\} .
$$

Then the set $\left\{\eta^{\prime \prime}(b): b \in \mathcal{D}_{0}\right\}$ is a core for $\widehat{\Delta}^{-1 / 2}$ and

$$
\left\|\widehat{\Delta}^{-1 / 2} \eta^{\prime \prime}(b)\right\|=\left\|\eta^{\prime \prime}\left(\kappa^{M}(b)^{*}\right)\right\|
$$

for any element $b \in \mathcal{D}_{0}$. 
We shall use the notation introduced in the previous section.

Lemma 5.2. Let $a$ be an element in $\mathcal{D}\left(\eta^{\prime \prime}\right)$. If $\eta^{\prime \prime}(a) \in \mathcal{D}\left(\Delta_{\text {rel }}^{1 / 2}\right)$, then $R(a) \in \mathcal{D}\left(\eta^{\prime \prime}\right)$ and

$$
\left\|\eta^{\prime \prime}(R(a))\right\|=\left\|\Delta_{\text {rel }}^{1 / 2} \eta^{\prime \prime}(a)\right\|
$$

Proof. If $a$ is an element in $\mathcal{D}\left(\eta^{\prime \prime}\right)$ satisfying $\eta^{\prime \prime}(a) \in \mathcal{D}\left(\Delta_{\text {rel }}^{1 / 2}\right)$, then $h\left(R(a)^{*} R(a)\right)=h^{L}\left(a a^{*}\right)=$ $\left\|\Delta_{\text {rel }}^{1 / 2} \eta^{\prime \prime}(a)\right\|^{2}<\infty$. Hence we obtain $R(a) \in \mathcal{D}\left(\eta^{\prime \prime}\right)$ and $\left\|\eta^{\prime \prime}(R(a))\right\|=\left\|\Delta_{\text {rel }}^{1 / 2} \eta^{\prime \prime}(a)\right\|$.

Lemma 5.3. Let a be an element in $\mathcal{D}\left(\eta^{\prime \prime}\right)$ with $\eta^{\prime \prime}(a) \in \mathcal{D}\left(\Delta^{1 / 2}\right)$. If $\eta^{\prime \prime}(a) \in \mathcal{D}\left(J \rho^{1 / 2} J\right)$, then $R(a)^{*} \in \mathcal{D}\left(\eta^{\prime \prime}\right)$ and

$$
\left\|\eta^{\prime \prime}\left(R(a)^{*}\right)\right\|=\left\|J \rho^{1 / 2} J \eta^{\prime \prime}(a)\right\|
$$

Proof. Since $\eta^{\prime \prime}(a) \in \mathcal{D}\left(\Delta^{1 / 2}\right)$, we have $a^{*} \in \mathcal{D}\left(\eta^{\prime \prime}\right)$ (cf. Theorem D.5) and

$$
\eta^{\prime \prime}\left(a^{*}\right)=J \Delta^{1 / 2} \eta^{\prime \prime}(a)=\Delta^{-1 / 2} J \eta^{\prime \prime}(a) ;
$$

hence, $\eta^{\prime \prime}\left(a^{*}\right) \in \mathcal{D}\left(\Delta^{1 / 2}\right)$ and $\Delta^{1 / 2} \eta^{\prime \prime}\left(a^{*}\right)=J \eta^{\prime \prime}(a)$. Assume that $\eta^{\prime \prime}(a) \in \mathcal{D}\left(J \rho^{1 / 2} J\right)$. Then $J \eta^{\prime \prime}(a) \in \mathcal{D}\left(\rho^{1 / 2}\right)$ and $\eta^{\prime \prime}\left(a^{*}\right) \in \mathcal{D}\left(\rho^{1 / 2} \Delta^{1 / 2}\right) \subset \mathcal{D}\left(\Delta_{\text {rel }}^{1 / 2}\right)$. Now we apply Lemma 5.2 with $a$ replaced by $a^{*}$ to obtain $R(a)^{*}=R\left(a^{*}\right) \in \mathcal{D}\left(\eta^{\prime \prime}\right)$ and

$$
\begin{aligned}
\left\|\eta^{\prime \prime}\left(R(a)^{*}\right)\right\| & =\left\|\eta^{\prime \prime}\left(R\left(a^{*}\right)\right)\right\|=\left\|\Delta_{\text {rel }}^{1 / 2} \eta^{\prime \prime}\left(a^{*}\right)\right\| \\
& =\left\|\rho^{1 / 2} \Delta^{1 / 2} \eta^{\prime \prime}\left(a^{*}\right)\right\|=\left\|\rho^{1 / 2} J \eta^{\prime \prime}(a)\right\|=\left\|J \rho^{1 / 2} J \eta^{\prime \prime}(a)\right\| .
\end{aligned}
$$

Next we have an improved version of the above statement, which plays the fundamental role in the subsequent argument.

Proposition 5.4. Let a be an element in $\mathcal{D}\left(\eta^{\prime \prime}\right)$. If $\eta^{\prime \prime}(a) \in \mathcal{D}\left(J \rho^{1 / 2} J\right)$, then $R(a)^{*} \in \mathcal{D}\left(\eta^{\prime \prime}\right)$ and

$$
\left\|\eta^{\prime \prime}\left(R(a)^{*}\right)\right\|=\left\|J \rho^{1 / 2} J \eta^{\prime \prime}(a)\right\| .
$$

Proof. For any $b \in \mathcal{D}\left(\eta^{\prime \prime}\right), b^{*} a \in \mathcal{D}\left(\eta^{\prime \prime}\right)$ and $\eta^{\prime \prime}\left(b^{*} a\right) \in \mathcal{D}\left(\Delta^{1 / 2}\right)$. Moreover due to the assumption $\eta^{\prime \prime}(a) \in \mathcal{D}\left(J \rho^{1 / 2} J\right)$ and the operator inclusion $J \rho^{1 / 2} J b^{*} \supset b^{*} J \rho^{1 / 2} J$, we have $\eta^{\prime \prime}\left(b^{*} a\right)=b^{*} \eta^{\prime \prime}(a) \in$ $\mathcal{D}\left(J \rho^{1 / 2} J\right)$. Therefore the element $b^{*} a$ satisfies the conditions of the previous Lemma and hence we have $R(b) R(a)^{*}=R\left(b^{*} a\right)^{*} \in \mathcal{D}\left(\eta^{\prime \prime}\right)$ and $\left\|\eta^{\prime \prime}\left(R\left(b^{*} a\right)^{*}\right)\right\|=\left\|J \rho^{1 / 2} J b^{*} \eta^{\prime \prime}(a)\right\|$. Therefore we have

$$
\left\|\eta^{\prime \prime}\left(R(b) R(a)^{*}\right)\right\|=\left\|b^{*} J \rho^{1 / 2} J \eta^{\prime \prime}(a)\right\| \leq\|b\|\left\|J \rho^{1 / 2} J \eta^{\prime \prime}(a)\right\| .
$$

Now we shall use an exact vector presentation (see Definition B.6] and Theorem B.7):

$$
\eta(a)=\sum_{n \in \mathbb{N}} a \Omega_{n}
$$

of the GNS mapping $\eta$. Then $\eta^{\prime \prime}\left(R\left(b^{*} a\right)^{*}\right)=\sum_{n \in \mathbb{N}} R(b) R\left(a^{*}\right) \Omega_{n}$, so for any natural $k$ and any $b \in \mathcal{D}\left(\eta^{\prime \prime}\right)$ with $\|b\| \leq 1$ we have

$$
\sum_{n=1}^{k}\left\|R(b) R\left(a^{*}\right) \Omega_{n}\right\|^{2} \leq\left\|J \rho^{1 / 2} J \eta^{\prime \prime}(a)\right\|^{2} .
$$

The set $\left\{b \in A^{\prime \prime}: b \in \mathcal{D}\left(\eta^{\prime \prime}\right),\|b\| \leq 1\right\}$ is weakly dense in the unit ball of the von Neumann algebra $A^{\prime \prime}$. It follows that for any natural $k$,

$$
\sum_{n=1}^{k}\left\|R\left(a^{*}\right) \Omega_{n}\right\|^{2} \leq\left\|J \rho^{1 / 2} J \eta^{\prime \prime}(a)\right\|^{2} .
$$

and the series $\sum_{n \in \mathbb{N}}\left\|R\left(a^{*}\right) \Omega_{n}\right\|^{2}$ is convergent. Remembering that our vector presentation is exact we see that $R\left(a^{*}\right) \in \mathcal{D}\left(\eta^{\prime \prime}\right)$. Now by taking elements $b$ in the unit ball of $\mathcal{D}\left(\eta^{\prime \prime}\right)$, we obtain

$$
\begin{aligned}
\left\|\eta^{\prime \prime}\left(R\left(a^{*}\right)\right)\right\| & =\sup _{\|b\| \leq 1}\left\|R(b) \eta^{\prime \prime}\left(R\left(a^{*}\right)\right)\right\| \\
& =\sup _{\|b\| \leq 1}\left\|b^{*} J \rho^{1 / 2} J \eta^{\prime \prime}(a)\right\|=\left\|J \rho^{1 / 2} J \eta^{\prime \prime}(a)\right\|
\end{aligned}
$$


Let

$$
\mathcal{D}_{1}=\left\{a \in \mathcal{D}\left(\eta^{\prime \prime}\right) \cap \mathcal{D}\left(\kappa^{M}\right): \begin{array}{c}
\eta^{\prime \prime}(a) \in \mathcal{D}\left(Q^{-1}\right) \cap \mathcal{D}\left(J \rho^{1 / 2} J\right) \\
\text { and } Q^{-1} \eta^{\prime \prime}(a) \in \mathcal{D}\left(J \rho^{1 / 2} J\right)
\end{array}\right\} .
$$

Proposition 5.5. $\mathcal{D}_{1} \subset \mathcal{D}_{0}$ and

$$
\left\|\eta^{\prime \prime}\left(\kappa^{M}(a)^{*}\right)\right\|=\left\|J \rho^{1 / 2} J Q^{-1} \eta^{\prime \prime}(a)\right\| \quad \text { for } \quad a \in \mathcal{D}_{1} .
$$

Proof. Suppose that $a \in \mathcal{D}_{1}$. We know (cf. Proposition 4.1) that $\tau_{i / 2}^{M}(a) \in \mathcal{D}\left(\eta^{\prime \prime}\right)$ and $\eta^{\prime \prime}\left(\tau_{i / 2}^{M}(a)\right)=\lambda^{i / 4} Q^{-1} \eta^{\prime \prime}(a)$. By our assumption, $Q^{-1} \eta^{\prime \prime}(a) \in \mathcal{D}\left(J \rho^{1 / 2} J\right)$. Applying Proposition 5.4 to the element $\tau_{i / 2}^{M}(a)$ instead of $a$, we see that $\kappa^{M}(a)^{*}=R\left(\tau_{i / 2}^{M}(a)\right)^{*} \in \mathcal{D}\left(\eta^{\prime \prime}\right)$. In this case equality (5.6) converts into (5.8).

Up to this point, it is not clear whether the sets $\mathcal{D}_{0}$ and $\mathcal{D}_{1}$ are not trivial. To show that they are large enough, we have to tame unbounded operators that appear in our theory. In order to tame $\rho$ and $\gamma$, for any $\varepsilon>0$ we introduce two elements in $A^{\prime \prime}$ as follows:

$$
\begin{aligned}
& \Gamma_{\varepsilon}:=\exp \left\{-\varepsilon\left((\log \rho)^{2}+\frac{1}{4}(\log \gamma)^{2}\right)\right\}, \\
& \Gamma_{\varepsilon}^{\prime}:=\exp \left\{-\varepsilon\left((\log \rho)^{2}-i(\log \rho)(\log \gamma)\right)\right\} .
\end{aligned}
$$

Notice that $0 \leq \Gamma_{\varepsilon} \leq 1$ and $\Gamma_{\varepsilon}^{\prime}$ is bounded. Moreover, $\Gamma_{\varepsilon}$ and $\Gamma_{\varepsilon}^{\prime}$ converges strongly to 1 as $\varepsilon$ tends to 0 .

Lemma 5.6. Let $a$ be an element in $\mathcal{D}\left(\eta^{\prime \prime}\right)$. Then $a \Gamma_{\varepsilon} \in \mathcal{D}\left(\eta^{\prime \prime}\right)$ and $\eta^{\prime \prime}\left(a \Gamma_{\varepsilon}\right)=J \Gamma_{\varepsilon}^{\prime} J \eta^{\prime \prime}(a)$.

Proof. Using (4.7), we can easily check that

$$
\sigma_{t}^{h}\left(\Gamma_{\varepsilon}\right)=\Delta^{i t} \Gamma_{\varepsilon} \Delta^{-i t}=\exp \left\{-\varepsilon\left(\left(\log \gamma^{t} \rho\right)^{2}+\frac{1}{4}(\log \gamma)^{2}\right)\right\} .
$$

The right hand side admits the holomorphic continuation for $t$ running over all $\mathbb{C}$. It means that $\Gamma_{\varepsilon} \in \mathcal{D}\left(\sigma_{i / 2}^{h}\right)$ and setting in the above formula $t=i / 2$ we obtain

$$
\sigma_{i / 2}\left(\Gamma_{\varepsilon}\right)=\exp \left\{-\varepsilon\left(\left(\log \rho+\frac{i}{2} \log \gamma\right)^{2}+\frac{1}{4}(\log \gamma)^{2}\right)\right\}=\left(\Gamma_{\varepsilon}^{\prime}\right)^{*}
$$

Now our statement follows immediately from Theorem D.7

In order to tame the effects coming from the unboundedness of $\log Q$, for any $\varepsilon>0$ and any $a \in M$ we set

$$
\mathcal{R}_{\varepsilon}(a)=\frac{1}{\sqrt{\varepsilon \pi}} \int_{\mathbb{R}} \tau_{t}^{M}\left(a \Gamma_{\varepsilon}\right) \lambda^{-t / 2} e^{-t^{2} / \varepsilon} d t .
$$

Remark 5.7. By (4.8), $\rho$ and $\gamma$ are $\left\{\tau_{t}\right\}_{t \in \mathbb{R}}$ invariant. So is $\Gamma_{\varepsilon}$. Therefore $\Gamma_{\varepsilon} \in \mathcal{D}\left(\tau_{i / 2}\right)$ and $\tau_{i / 2}\left(\Gamma_{\varepsilon}\right)=\Gamma_{\varepsilon}$. Applying $R$ to the both sides, we conclude that $\Gamma_{\varepsilon} \in \mathcal{D}\left(\kappa^{M}\right)$ and $\kappa^{M}\left(\Gamma_{\varepsilon}\right)=R\left(\Gamma_{\varepsilon}\right)=$ $\Gamma_{\varepsilon}$. The latter equality follows from Proposition 4.6 Since $\mathcal{D}\left(\kappa^{M}\right)$ is a subalgebra and that $\kappa^{M}$ is antimultiplicative, we see that $a \Gamma_{\varepsilon} \in \mathcal{D}\left(\kappa^{M}\right)$ and $\kappa^{M}\left(a \Gamma_{\varepsilon}\right)^{*}=\kappa^{M}(a)^{*} \Gamma_{\varepsilon}$ for any $a \in \mathcal{D}\left(\kappa^{M}\right)$. Using this result together with the closedness of $\kappa^{M}$, one can easily show that $\mathcal{R}_{\varepsilon}(a) \in \mathcal{D}\left(\kappa^{M}\right)$ and

$$
\mathcal{R}_{\varepsilon}\left(\kappa^{M}(a)^{*}\right)=\kappa^{M}\left(\mathcal{R}_{\varepsilon}(a)\right)^{*}
$$

for any $a \in \mathcal{D}\left(\kappa^{M}\right)$.

\section{Lemma 5.8.}

1. For any $a \in M, \mathcal{R}_{\varepsilon}(a)$ converges strongly to a when $\varepsilon \longrightarrow 0$.

2. Let $a \in \mathcal{D}\left(\eta^{\prime \prime}\right)$ and $\varepsilon>0$. Then $\mathcal{R}_{\varepsilon}(a) \in \mathcal{D}_{1}$ and

$$
\eta^{\prime \prime}\left(\mathcal{R}_{\varepsilon}(a)\right)=\exp \left\{-\varepsilon(\log Q)^{2}\right\} J \Gamma_{\varepsilon}^{\prime} J \eta^{\prime \prime}(a) .
$$

In particular $\eta^{\prime \prime}\left(\mathcal{R}_{\varepsilon}(a)\right)$ converges in norm to $\eta^{\prime \prime}(a)$ when $\varepsilon \longrightarrow 0$.

3. The set $\mathcal{D}_{1}$ is a core for $\eta^{\prime \prime}$. 
Proof. Ad 1. One can easily verify that

$$
\delta_{\varepsilon}(t)=\frac{1}{\sqrt{\varepsilon \pi}} \lambda^{-t / 2} e^{-t^{2} / \varepsilon}
$$

is a $\delta$-like sequence when $\varepsilon \rightarrow 0$. More precisely: $\delta_{\varepsilon}(t)>0$ for all $t \in \mathbb{R}$ and $\varepsilon>0, \int_{\mathbb{R}} \delta_{\varepsilon}(t) d t=1$ and for any neighborhood $\mathcal{O}$ of 0 in $\mathbb{R}, \int_{\mathbb{R} \backslash \mathcal{O}} \delta_{\varepsilon}(t) d t \longrightarrow 0$ when $\varepsilon \rightarrow 0$. Therefore for any continuous bounded function $\varphi$ on $\mathbb{R}$ we have

$$
\lim _{\varepsilon \rightarrow 0} \int_{\mathbb{R}} \varphi(t) \delta_{\varepsilon}(t) d t=\varphi(0) .
$$

Let $x \in \mathcal{H}$. Then

$$
\begin{aligned}
\left\|\mathcal{R}_{\varepsilon}(a) x-a x\right\| & =\left\|\int_{\mathbb{R}}\left(\tau_{t}^{M}(a) \Gamma_{\varepsilon} x-a x\right) \delta_{\varepsilon}(t) d t\right\| \\
& \leq \int_{\mathbb{R}}\left\|\tau_{t}^{M}(a)\left(\Gamma_{\varepsilon} x-x\right)\right\| \delta_{\varepsilon}(t) d t+\int_{\mathbb{R}}\left\|\tau_{t}^{M}(a) x-a x\right\| \delta_{\varepsilon}(t) d t \\
& \leq\|a\|\left\|\Gamma_{\varepsilon} x-x\right\|+\int_{\mathbb{R}}\left\|\tau_{t}^{M}(a) x-a x\right\| \delta_{\varepsilon}(t) d t
\end{aligned}
$$

The first term on the right hand side converges to 0 as $\varepsilon$ goes to 0 . Inserting in (5.13), $\varphi(t)=$ $\left\|\tau_{t}^{M}(a) x-a x\right\|$ we see that also the second term converges to 0 . It shows that $\mathcal{R}_{\varepsilon}(a) x$ converges in norm to $a x$.

Ad 2. Assume that $a \in \mathcal{D}\left(\eta^{\prime \prime}\right)$. We already know by Lemma [5.6 that $a \Gamma_{\varepsilon} \in \mathcal{D}\left(\eta^{\prime \prime}\right)$ and $\eta^{\prime \prime}\left(a \Gamma_{\varepsilon}\right)=J \Gamma_{\varepsilon}^{\prime} J \eta^{\prime \prime}(a)$. By (4.1), $\tau_{t}^{M}\left(a \Gamma_{\varepsilon}\right) \in \mathcal{D}\left(\eta^{\prime \prime}\right)$ and $\eta^{\prime \prime}\left(\tau_{t}^{M}\left(a \Gamma_{\varepsilon}\right)\right)=\lambda^{t / 2} Q^{2 i t} J \Gamma_{\varepsilon}^{\prime} J \eta^{\prime \prime}(a)$. Therefore

$$
\begin{aligned}
\frac{1}{\sqrt{\varepsilon \pi}} \int_{\mathbb{R}} \eta^{\prime \prime}\left(\tau_{t}^{M}\left(a \Gamma_{\varepsilon}\right)\right) \lambda^{-t / 2} e^{-t^{2} / \varepsilon} d t & =\frac{1}{\sqrt{\varepsilon \pi}} \int_{\mathbb{R}} Q^{2 i t} e^{-t^{2} / \varepsilon} J \Gamma_{\varepsilon}^{\prime} J \eta^{\prime \prime}(a) d t \\
& =e^{-\varepsilon(\log Q)^{2}} J \Gamma_{\varepsilon}^{\prime} J \eta^{\prime \prime}(a) .
\end{aligned}
$$

Remembering that $\eta^{\prime \prime}$ is closed, we conclude that

$$
\mathcal{R}_{\varepsilon}(a)=\frac{1}{\sqrt{\varepsilon \pi}} \int_{\mathbb{R}} \tau_{t}^{M}\left(a \Gamma_{\varepsilon}\right) \lambda^{-t / 2} e^{-t^{2} / \varepsilon} d t \in \mathcal{D}\left(\eta^{\prime \prime}\right)
$$

and $\eta^{\prime \prime}\left(\mathcal{R}_{\varepsilon}(a)\right)=e^{-\varepsilon(\log Q)^{2}} J \Gamma_{\varepsilon}^{\prime} J \eta^{\prime \prime}(a)$. Due to the dumping factor $e^{-\varepsilon(\log Q)^{2}} J \Gamma_{\varepsilon}^{\prime} J$, the vector $\eta^{\prime \prime}\left(\mathcal{R}_{\varepsilon}(a)\right)$ belongs to $\mathcal{D}\left(Q^{-1}\right)$ and the vectors $\eta^{\prime \prime}\left(\mathcal{R}_{\varepsilon}(a)\right)$ and $Q^{-1} \eta^{\prime \prime}\left(\mathcal{R}_{\varepsilon}(a)\right)$ belong to $\mathcal{D}\left(J \rho^{1 / 2} J\right)$.

One can easily verify that $\mathcal{R}_{\varepsilon}(a)$ is an entire analytic element for the scaling group. Therefore $\mathcal{R}_{\varepsilon}(a) \in \mathcal{D}\left(\tau_{i / 2}^{M}\right)=\mathcal{D}\left(\kappa^{M}\right)$. It means that $\mathcal{R}_{\varepsilon}(a) \in \mathcal{D}_{1}$.

Ad 3. Follows immediately from the first two assertions.

Now we recall a fact concerning a core for positive self-adjoint operators

Lemma 5.9. Let $H$ be a strictly positive self-adjoint operator on $\mathcal{H}$. Let $K$ be a bounded operator such that $K \mathcal{H}$ is dense in $\mathcal{H}, K \mathcal{H} \subset \mathcal{D}(H), H K \in \mathcal{L}(\mathcal{H})$ and $H K x=K H x$ for $x \in \mathcal{D}(H)$. Then, for any dense linear subset $\mathcal{D}$ of $\mathcal{H}, K \mathcal{D}$ is a core for $H$.

Proof. It suffices to show that the set $(H+1) K \mathcal{D}$ is dense in $\mathcal{H}$. Suppose that $y$ is orthogonal to the above set. Then $(y \mid(H+1) K x)=0$ holds for any vector $x$ in $\mathcal{D}$. Since $H K \in \mathcal{L}(\mathcal{H})$, it holds for $x$ in $\mathcal{H}$. Therefore $(y \mid K(H+1) x)=(y \mid(H+1) K x)=0$ holds for any $x \in \mathcal{D}(H)$. Since $(H+1) \mathcal{D}(H)=\mathcal{H}$ and $K$ has a dense range, it follows that $y=0$.

Now we shall use the operator $\widehat{\Delta}$ introduced by (5.1). Clearly, $\widehat{\Delta}^{-1 / 2}=\overline{J \rho^{1 / 2} J \circ Q^{-1}}$.

Proposition 5.10. The set $\left\{\eta^{\prime \prime}(b): b \in \mathcal{D}_{1}\right\}$ is a core for $\widehat{\Delta}^{-1 / 2}$.

Proof. At first we notice that $\left\{\eta^{\prime \prime}(b): b \in \mathcal{D}_{1}\right\} \subset \mathcal{D}\left(\widehat{\Delta}^{-1 / 2}\right)$ by (5.7). We use the previous lemma with $H=\widehat{\Delta}^{-1 / 2}=\overline{J \rho^{1 / 2} J \circ Q^{-1}}, K=J \Gamma_{1}^{\prime} J e^{-(\log Q)^{2}}$ and $\mathcal{D}=\left\{\eta^{\prime \prime}(a): a \in \mathcal{D}\left(\eta^{\prime \prime}\right)\right\}$. It is clear 
that $K$ is bounded and the range is dense in $\mathcal{H}$. Let $x \in \mathcal{D}(H)$. There exists a sequence $\left\{x_{n}\right\}_{n \in \mathbb{N}}$ in $\mathcal{D}\left(J \rho^{1 / 2} J \circ Q^{-1}\right)$ such that $x_{n} \rightarrow x$ and $H x_{n} \rightarrow H x$ in norm. For $x_{n}$ we see that

$$
\begin{aligned}
K H x_{n} & =J \Gamma_{1}^{\prime} J e^{-(\log Q)^{2}} J \rho^{1 / 2} J Q^{-1} x_{n}=J \rho^{1 / 2} \Gamma_{1}^{\prime} J e^{-(\log Q)^{2}} Q^{-1} x_{n} \\
& =J \rho^{1 / 2} \Gamma_{1}^{\prime} J Q^{-1} e^{-(\log Q)^{2}} x_{n}=J \rho^{1 / 2} J Q^{-1} J \Gamma_{1}^{\prime} J e^{-(\log Q)^{2}} x_{n}=H K x_{n} .
\end{aligned}
$$

Since $K x_{n} \rightarrow K x$ and $H K x_{n}=K H x_{n} \rightarrow K H x$, the closedness of $H$ implies that $K x \in \mathcal{D}(H)$ and $H K x=K H x$. Hence $K \mathcal{H}$ is contained in the domain of $H$ and the operator $H K$ is bounded on $\mathcal{H}$. Thus above lemma tells us that $K \mathcal{D}$ is a core for $\widehat{\Delta}^{-1 / 2}$. On the other hand, according to Lemma 5.8 (with $\varepsilon=1$ ), KD is contained in the set $\left\{\eta^{\prime \prime}(b): b \in \mathcal{D}_{1}\right\}$ and assertion follows.

We are now ready to prove the main theorem of this section.

Proof of Theorem [5.1 First we prove that

$$
\left\{\eta^{\prime \prime}(b): b \in \mathcal{D}_{0}\right\} \subset \mathcal{D}\left(\widehat{\Delta}^{-1 / 2}\right)
$$

Let $b \in \mathcal{D}_{0}$. Then, Lemma 5.8 shows that $\mathcal{R}_{\varepsilon}(b) \in \mathcal{D}_{1}$ for any $\varepsilon>0$. Inserting $a=\mathcal{R}_{\varepsilon}(b)-\mathcal{R}_{\varepsilon^{\prime}}(b)$ in (5.8) and using (5.11), we get

$$
\left\|\widehat{\Delta}^{-1 / 2} \eta^{\prime \prime}\left(\mathcal{R}_{\varepsilon}(b)\right)-\widehat{\Delta}^{-1 / 2} \eta^{\prime \prime}\left(\mathcal{R}_{\varepsilon^{\prime}}(b)\right)\right\|=\left\|\eta^{\prime \prime}\left(\mathcal{R}_{\varepsilon}\left(\kappa^{M}(b)\right)^{*}\right)-\eta^{\prime \prime}\left(\mathcal{R}_{\varepsilon^{\prime}}\left(\kappa^{M}(b)\right)^{*}\right)\right\|
$$

for any $\varepsilon, \varepsilon^{\prime}>0$. According to Assertion 2 of Lemma [5.8 $\eta^{\prime \prime}\left(\mathcal{R}_{\varepsilon}(b)\right)$ and $\eta^{\prime \prime}\left(\mathcal{R}_{\varepsilon}\left(\kappa^{M}(b)^{*}\right)\right)$ converge in norm to $\eta^{\prime \prime}(b)$ and $\eta^{\prime \prime}\left(\kappa^{M}(b)^{*}\right)$ respectively as $\varepsilon$ tends to 0 . By the Cauchy criterion, $\widehat{\Delta}^{-1 / 2} \eta^{\prime \prime}\left(\mathcal{R}_{\varepsilon}(b)\right)$ has a norm limit when $\varepsilon$ tends to 0 . Since $\widehat{\Delta}^{-1 / 2}$ is closed, we see that $\eta^{\prime \prime}(b) \in \mathcal{D}\left(\widehat{\Delta}^{-1 / 2}\right)$ and (5.15) follows.

The rest is easy. Remembering that $\mathcal{D}_{1} \subset \mathcal{D}_{0}$ (cf. Proposition 5.5) and using Proposition 5.10 we see that the set $\left\{\eta^{\prime \prime}(b): b \in \mathcal{D}_{0}\right\}$ is a core for $\widehat{\Delta}^{-1 / 2}$. Let $b \in \mathcal{D}_{0}$. Replacing $a$ in (5.8) by $\mathcal{R}_{\varepsilon}(b)$ and setting $\varepsilon \rightarrow 0$, we obtain the norm equality (5.3).

Now, we shall consider the conjugate linear operator

$$
\widehat{J}: \widehat{\Delta}^{-1 / 2} \eta^{\prime \prime}(b) \mapsto \eta^{\prime \prime}\left(\kappa^{M}(b)^{*}\right),
$$

where $b$ runs over $\mathcal{D}_{0}$. By Theorem [5.1] $\widehat{J}$ is a densely defined isometry. By the same letter we denote its continuous extension on the whole $\mathcal{H}$. Let

$$
\widehat{F}=\widehat{J} \widehat{\Delta}^{-1 / 2} \text {. }
$$

Then

$$
\widehat{F} \eta^{\prime \prime}(b)=\eta^{\prime \prime}\left(\kappa^{M}(b)^{*}\right)
$$

for all $b \in \mathcal{D}_{0}$. Moreover the set $\left\{\eta^{\prime \prime}(b): b \in \mathcal{D}_{0}\right\}$ is a core for $\widehat{F}$, because it is a core for $\widehat{\Delta}^{-1 / 2}$. In other words, $\widehat{F}$ is the closure of the mapping

$$
\eta^{\prime \prime}(b) \mapsto \eta^{\prime \prime}\left(\kappa^{M}(b)^{*}\right)
$$

where $b$ runs over $\mathcal{D}_{0}$.

The mapping $b \mapsto \kappa^{M}(b)^{*}$ is an involution acting on $\mathcal{D}_{0}$. Therefore $\widehat{F}$ is also an involution. Clearly (5.18) is the polar decomposition of $\widehat{F}$. Now the relation $\widehat{F}^{-1}=\widehat{F}$ implies that

$$
\widehat{J}^{2}=1 \quad \text { and } \quad \widehat{J} \widehat{\Delta} \widehat{J}=\widehat{\Delta}^{-1} .
$$

It means that the operator $\widehat{J}$ is an antiunitary involution.

Theorem 5.11. We have:

$$
\begin{gathered}
R(a)=\widehat{J} a^{*} \widehat{J}, \\
(J \otimes \widehat{J}) W(J \otimes \widehat{J})=W^{*},
\end{gathered}
$$

The first formula holds for all $a \in A$. 
Proof. Ad (5.21). Let $a \in \mathcal{D}(\kappa)$ and $b \in \mathcal{D}_{0}$. Using (5.2), one can easily check that $a b \in \mathcal{D}_{0}$. Therefore $a \eta^{\prime \prime}(b)=\eta^{\prime \prime}(a b) \in \mathcal{D}\left(\widehat{\Delta}^{-1 / 2}\right)$ and replacing $b$ in (5.17) by $a b$, we get

$$
\begin{aligned}
\widehat{J} \widehat{\Delta}^{-1 / 2} a \eta^{\prime \prime}(b) & =\widehat{J} \widehat{\Delta}^{-1 / 2} \eta^{\prime \prime}(a b)=\eta^{\prime \prime}\left(\kappa^{M}(a b)^{*}\right) \\
& =\eta^{\prime \prime}\left(\kappa(a)^{*} \kappa^{M}(b)^{*}\right)=\kappa(a)^{*} \widehat{J}^{-1 / 2} \eta^{\prime \prime}(b) .
\end{aligned}
$$

Since $\left\{\eta^{\prime \prime}(b): b \in \mathcal{D}_{0}\right\}$ is a core for $\widehat{\Delta}^{-1 / 2}$, we see that

$$
\widehat{J} \kappa(a)^{*} \widehat{J} \widehat{\Delta}^{-1 / 2} \subset \widehat{\Delta}^{-1 / 2} a .
$$

On the other hand, using (2.2), one can show that $\tau_{i / 2}(a) Q^{-1} \subset Q^{-1} a$. For $x \in \mathcal{D}\left(J \rho^{1 / 2} J Q^{-1}\right)$ we see that

$$
\tau_{i / 2}(a) J \rho^{1 / 2} J Q^{-1} x=J \rho^{1 / 2} J \tau_{i / 2}(a) Q^{-1} x=J \rho^{1 / 2} J Q^{-1} a x=\widehat{\Delta}^{-1 / 2} a x .
$$

Hence $\tau_{i / 2}(a) \widehat{\Delta}^{-1 / 2} \subset \widehat{\Delta}^{-1 / 2} a$. Comparing this inclusion with (5.24), we obtain

$$
\widehat{J} \kappa(a)^{*} \widehat{J}=\tau_{i / 2}(a) .
$$

Replacing $a$ by $\kappa^{-1}(a)$, we get

$$
\widehat{J} a^{*} \widehat{J}=R(a)
$$

for all $a$ in the range of $\kappa$. To end the proof we notice that the range of $\kappa$ equal to $\mathcal{D}(\kappa)^{*}$ is dense in $A$.

Ad (5.22). Remembering that $R(W(x, y))=W(J y, J x)$ (cf. Assertion 2 of Proposition 1.11) and $R(a)=\widehat{J} a^{*} \widehat{J}$, we compute

$$
\begin{aligned}
(J x \otimes \widehat{J} z \mid W(J y \otimes \widehat{J} u)) & =(\widehat{J} z \mid W(J x, J y) \widehat{J} u) \\
& =(\widehat{J} z \mid R(W(y, x)) \widehat{J} u)=\left(\widehat{J} z \mid \widehat{J} W(y, x)^{*} u\right)=(u \mid W(y, x) z) \\
& =(y \otimes u \mid W(x \otimes z))=\left(J x \otimes \widehat{J} z \mid(J \otimes \widehat{J}) W^{*}(y \otimes u)\right) .
\end{aligned}
$$

Thus we have $W(J \otimes \widehat{J})=(J \otimes \widehat{J}) W^{*}$.

Ad (5.23). By (3.16) it follows immediately from (5.22).

Remark 5.12. By definitions (5.1) and (5.17), we see that $Q^{i t}$ commute with $\widehat{\Delta}$ and $\widehat{J}$ for all $t \in \mathbb{R}$. It implies that $Q$ and $\widehat{\Delta}$ strongly commute and

$$
\widehat{J} Q \widehat{J}=Q^{-1} \text {. }
$$

In Section 3 we used the transpose mapping acting on $\mathcal{L}(\mathcal{H})$. It was defined in the following way :

$$
m^{\top}=J m^{*} J
$$

for any $m \in \mathcal{L}(\mathcal{H})$. Formula $(5.23)$ shows that this transpose maps $\widehat{A}$ onto itself. Remembering that $\top$ is an involution, we have $W^{\top \top \otimes i d}=W$. Comparing this formula with (3.23), we see that $\widehat{R}=\top$. More explicitly

$$
\widehat{R}(b)=J b^{*} J
$$

for all $b \in \widehat{A}$.

Proposition 5.13. Let $\eta_{L}$ be a linear mapping from $A$ to $\mathcal{H}$ with the domain $\mathcal{D}\left(\eta_{L}\right)=R\left(\mathcal{D}(\eta)^{*}\right)=$ $\left\{a \in A: R\left(a^{*}\right) \in \mathcal{D}(\eta)\right\}$ defined by the formula

$$
\eta_{L}(a)=\widehat{J} \eta\left(R\left(a^{*}\right)\right) .
$$

Then $\eta_{L}$ is a closed GNS-mapping and the weight corresponding to $\eta_{L}$ coincides with $h^{L}=h^{\circ} R$.

Proof. Let $a \in \mathcal{D}\left(\eta_{L}\right)$ and $b \in A$. Then $R\left(a^{*}\right) \in \mathcal{D}(\eta)$ and $R\left((b a)^{*}\right)=R\left(b^{*}\right) R\left(a^{*}\right) \in \mathcal{D}(\eta)$. Therefore $b a \in \mathcal{D}\left(\eta_{L}\right)$ and using (5.21) in the last step, we obtain

$$
\eta_{L}(b a)=\widehat{J} \eta\left(R\left(b^{*}\right) R\left(a^{*}\right)\right)=\widehat{J} R\left(b^{*}\right) \widehat{J} \widehat{J} \eta\left(R\left(a^{*}\right)\right)=b \eta_{L}(a) .
$$

It shows that $\eta_{L}$ is a GNS-mapping. The closedness of $\eta_{L}$ follows from that of $\eta$. To show the last statement we notice that

$$
(h \circ R)\left(a^{*} a\right)<\infty \Leftrightarrow h\left(R(a) R\left(a^{*}\right)\right)<\infty \Leftrightarrow R\left(a^{*}\right) \in \mathcal{D}(\eta) \Leftrightarrow a \in \mathcal{D}\left(\eta_{L}\right) .
$$


Moreover, in this case

$$
(h \circ R)\left(a^{*} a\right)=\left\|\eta\left(R\left(a^{*}\right)\right)\right\|^{2}=\left\|\widehat{J} \eta\left(R\left(a^{*}\right)\right)\right\|^{2}=\left\|\eta_{L}(a)\right\|^{2} .
$$

Let

$$
\eta(a)=\sum_{k}^{\oplus} a \Omega_{k}
$$

where $a \in \mathcal{D}(\eta)$, be a vector presentation of the GNS map $\eta$ (see Definition B.4 and Theorem B.7). Then for any $a \in \mathcal{D}\left(\eta_{L}\right)$ we have:

$$
\eta_{L}(a)=\widehat{J} \eta\left(R(a)^{*}\right)=\widehat{J} \sum_{k}^{\oplus} R(a)^{*} \Omega_{k}=\widehat{J} \sum_{k}^{\oplus} \widehat{J} a \widehat{J} \Omega_{k}=\sum_{k}^{\oplus} a \widehat{J} \Omega_{k} .
$$

Clearly

$$
\eta_{L}(a)=\sum_{k}^{\oplus} a \widehat{J} \Omega_{k}
$$

is a vector presentation of the GNS map $\eta_{L}$. If (5.28) is exact, so is (5.29).

Proposition 5.14. For any $a \in \mathcal{D}\left(\eta^{\prime \prime}\right)$ we have

$$
\left(a \in \mathcal{D}\left(\eta_{L}^{\prime \prime}\right)\right) \Longleftrightarrow\left(\eta^{\prime \prime}(a) \in \mathcal{D}\left(J \rho^{1 / 2} J\right)\right)
$$

If this is the case, then

$$
\eta_{L}^{\prime \prime}(a)=\lambda^{-i / 4} J \rho^{1 / 2} J \eta^{\prime \prime}(a) .
$$

Furthermore $\mathcal{D}\left(\eta^{\prime \prime}\right) \cap \mathcal{D}\left(\eta_{L}^{\prime \prime}\right)$ is a core for $\eta^{\prime \prime}$ and $\eta_{L}^{\prime \prime}$.

Proof. We know that $R$ is normal. Passing to the weak closures in (5.27), we obtain $\mathcal{D}\left(\eta_{L}^{\prime \prime}\right)=$ $\left\{a \in M: R(a)^{*} \in \mathcal{D}\left(\eta^{\prime \prime}\right)\right\}$ and

$$
\eta_{L}^{\prime \prime}(a)=\widehat{J} \eta^{\prime \prime}\left(R(a)^{*}\right)
$$

Using Proposition 5.4 we see that

$$
\left(\eta^{\prime \prime}(a) \in \mathcal{D}\left(J \rho^{1 / 2} J\right)\right) \Longrightarrow\left(\begin{array}{c}
a \in \mathcal{D}\left(\eta_{L}^{\prime \prime}\right) \quad \text { and } \\
\left\|\eta_{L}^{\prime \prime}(a)\right\|=\left\|J \rho^{1 / 2} J \eta^{\prime \prime}(a)\right\| .
\end{array}\right)
$$

Therefore there exists a linear isometry $V$ such that

$$
\eta_{L}^{\prime \prime}(a)=V J \rho^{1 / 2} J \eta^{\prime \prime}(a)
$$

for any $a \in \mathcal{D}\left(\eta^{\prime \prime}\right)$ such that $\eta^{\prime \prime}(a) \in \mathcal{D}\left(J \rho^{1 / 2} J\right)$. We have to show that $V=\lambda^{-i / 4} 1$.

Let $b \in \mathcal{D}_{1}$ (cf. (5.7)). Then $\tau_{i / 2}(b) \in \mathcal{D}\left(\eta^{\prime \prime}\right)$ and $\eta^{\prime \prime}\left(\tau_{i / 2}(b)\right)=\lambda^{i / 4} Q^{-1} \eta^{\prime \prime}(b) \in \mathcal{D}\left(J \rho^{1 / 2} J\right)$. Replacing $a$ in (5.32) by $\tau_{i / 2}(b)$ and using (5.1), we obtain

$$
\eta_{L}^{\prime \prime}\left(\tau_{i / 2}(b)\right)=\lambda^{i / 4} V J \rho^{1 / 2} J Q^{-1} \eta^{\prime \prime}(b)=\lambda^{i / 4} V \widehat{\Delta}^{-1 / 2} \eta^{\prime \prime}(b) .
$$

On the other hand

$$
\eta_{L}^{\prime \prime}\left(\tau_{i / 2}(b)\right)=\widehat{J} \eta^{\prime \prime}\left(\left(R \circ \tau_{i / 2}(b)\right)^{*}\right)=\widehat{J} \eta^{\prime \prime}\left(\kappa^{M}(b)^{*}\right) .
$$

We know that $\widehat{J}$ is an involution. Combining the above formula with (5.17), we get $\eta_{L}^{\prime \prime}\left(\tau_{i / 2}(b)\right)=$ $\widehat{\Delta}^{-1 / 2} \eta^{\prime \prime}(b)$. Therefore from (5.33) we have $V \widehat{\Delta}^{-1 / 2} \eta^{\prime \prime}(b)=\lambda^{-i / 4} \widehat{\Delta}^{-1 / 2} \eta^{\prime \prime}(b)$.

By Proposition 5.10 the set $\left\{\eta^{\prime \prime}(b): b \in \mathcal{D}_{1}\right\}$ is a core for $\widehat{\Delta}^{-1 / 2}$. Hence $\left\{\widehat{\Delta}^{-1 / 2} \eta^{\prime \prime}(b): b \in \mathcal{D}_{1}\right\}$ is dense in $\mathcal{H}, V=\lambda^{-i / 4} 1$ and (5.31) follows.

Assume now that $a \in \mathcal{D}\left(\eta^{\prime \prime}\right)$. By Lemma [5.6 we see that $a \Gamma_{\varepsilon} \in \mathcal{D}\left(\eta^{\prime \prime}\right)$ and

$$
\eta^{\prime \prime}\left(a \Gamma_{\varepsilon}\right)=J \Gamma_{\varepsilon}^{\prime} J \eta^{\prime \prime}(a) \in \mathcal{D}\left(J \rho^{1 / 2} J\right) .
$$

Using the part of the proposition proved so far, we obtain $a \Gamma_{\varepsilon} \in \mathcal{D}\left(\eta_{L}^{\prime \prime}\right)$ and

$$
\eta_{L}^{\prime \prime}\left(a \Gamma_{\varepsilon}\right)=\lambda^{-i / 4} J \rho^{1 / 2} J J \Gamma_{\varepsilon}^{\prime} J \eta^{\prime \prime}(a) .
$$

If moreover $a \in \mathcal{D}\left(\eta_{L}^{\prime \prime}\right)$, then $R(a)^{*} \in \mathcal{D}\left(\eta^{\prime \prime}\right)$ and

$$
\begin{aligned}
\eta_{L}^{\prime \prime}\left(a \Gamma_{\varepsilon}\right) & =\widehat{J} \eta^{\prime \prime}\left(R\left(a \Gamma_{\varepsilon}\right)^{*}\right)=\widehat{J} \eta^{\prime \prime}\left(R(a)^{*} \Gamma_{\varepsilon}\right) \\
& =\widehat{J} J \Gamma_{\varepsilon}^{\prime} J \eta^{\prime \prime}\left(R(a)^{*}\right)=\widehat{J} J \Gamma_{\varepsilon}^{\prime} J \widehat{J} \eta_{L}^{\prime \prime}(a)
\end{aligned}
$$


It shows that

$$
\widehat{J} J \Gamma_{\varepsilon}^{\prime} J \widehat{J} \eta_{L}^{\prime \prime}(a)=\lambda^{-i / 4} J \rho^{1 / 2} J J \Gamma_{\varepsilon}^{\prime} J \eta^{\prime \prime}(a) .
$$

When $\varepsilon \rightarrow 0$, then $J \Gamma_{\varepsilon}^{\prime} J \eta^{\prime \prime}(a) \rightarrow \eta^{\prime \prime}(a)$ and $\widehat{J} J \Gamma_{\varepsilon}^{\prime} J \widehat{J} \eta_{L}^{\prime \prime}(a) \rightarrow \eta_{L}^{\prime \prime}(a)$. Since $J \rho^{1 / 2} J$ is closed, we see that $\eta^{\prime \prime}(a) \in \mathcal{D}\left(J \rho^{1 / 2} J\right)$. The proof of (5.30) is complete.

By Lemma $\left[5.8\right.$ the set $\mathcal{D}_{1}$ is a core for $\eta^{\prime \prime}$. According to definition (5.7), $\eta^{\prime \prime}(a) \in \mathcal{D}\left(J \rho^{1 / 2} J\right)$ for any $a \in \mathcal{D}_{1}$. Using (5.30), we see that $\mathcal{D}_{1} \subset \mathcal{D}\left(\eta_{L}^{\prime \prime}\right)$. Therefore $\mathcal{D}_{1} \subset \mathcal{D}\left(\eta^{\prime \prime}\right) \cap \mathcal{D}\left(\eta_{L}^{\prime \prime}\right)$ and hence $\mathcal{D}\left(\eta^{\prime \prime}\right) \cap \mathcal{D}\left(\eta_{L}^{\prime \prime}\right)$ is a core for $\eta^{\prime \prime}$. By the symmetry it is also a core for $\eta_{L}^{\prime \prime}$.

With the vector presentations (5.28) and (5.29), formula (5.31) takes the form:

$$
\sum_{k}^{\oplus} a \widehat{J} \Omega_{k}=\lambda^{-i / 4} J \rho^{1 / 2} J \sum_{k}^{\oplus} a \Omega_{k} .
$$

We shall need the following extended version of this formula

Proposition 5.15. Let $\mathcal{H}_{1}$ be a Hilbert space, $c \in M \bar{\otimes} \mathcal{L}\left(\mathcal{H}_{1}\right)$ and $y \in \mathcal{H}_{1}$. Assume that the vector presentation (5.28) is exact, the series $\sum_{k}^{\oplus} c\left(\Omega_{k} \otimes y\right)$ is norm convergent and that

$$
\sum_{k}^{\oplus} c\left(\Omega_{k} \otimes y\right) \in \mathcal{D}\left(J \rho^{1 / 2} J \otimes 1\right) .
$$

Then

$$
\sum_{k}^{\oplus} c\left(\widehat{J} \Omega_{k} \otimes y\right)=\lambda^{-i / 4}\left(J \rho^{1 / 2} J \otimes 1\right) \sum_{k}^{\oplus} c\left(\Omega_{k} \otimes y\right)
$$

where the series on the left hand side is norm convergent.

Proof. We choose an orthonormal basis $\left\{e_{s}\right\}_{s=1,2, \ldots}$ in $\mathcal{H}_{1}$. Then there exists a sequence $\left\{c_{s}\right\}_{s=1,2, \ldots}$ of elements of $M$ such that

$$
c(x \otimes y)=\sum_{s}^{\oplus} c_{s} x \otimes e_{s}
$$

for all $x \in \mathcal{H}$. Inserting $x=\Omega_{k}$ and summing over $k$ we get a norm converging double series

$$
\sum_{k}^{\oplus} c\left(\Omega_{k} \otimes y\right)=\sum_{s}^{\oplus}\left(\sum_{k}^{\oplus} c_{s} \Omega_{k} \otimes e_{s}\right) .
$$

In particular for all $s$ the series $\sum_{k}^{\oplus} c_{s} \Omega_{k}$ are norm convergent. Therefore (cf. Definition [B.6) $c_{s} \in \mathcal{D}\left(\eta^{\prime \prime}\right)$ and

$$
\sum_{k}^{\oplus} c\left(\Omega_{k} \otimes y\right)=\sum_{s}^{\oplus} \eta^{\prime \prime}\left(c_{s}\right) \otimes e_{s}
$$

Assumption (5.34) shows now that $\eta^{\prime \prime}\left(c_{s}\right) \in \mathcal{D}\left(J \rho^{1 / 2} J\right)$ and $c_{s} \in \mathcal{D}\left(\eta_{L}^{\prime \prime}\right)$ for all $s$. Now we have:

$$
\begin{aligned}
\left(\lambda^{-i / 4} J \rho^{1 / 2} J \otimes 1\right) \sum_{k}^{\oplus} c\left(\Omega_{k} \otimes y\right) & =\sum_{s}^{\oplus} \lambda^{-i / 4} J \rho^{1 / 2} J \eta^{\prime \prime}\left(c_{s}\right) \otimes e_{s}=\sum_{s}^{\oplus} \eta_{L}^{\prime \prime}\left(c_{s}\right) \otimes e_{s} \\
& =\sum_{s}^{\oplus} \sum_{k}^{\oplus} c_{s} \widehat{J} \Omega_{k} \otimes e_{s}=\sum_{k}^{\oplus} c\left(\widehat{J} \Omega_{k} \otimes y\right),
\end{aligned}
$$

where in the last step we used (5.36).

We end this section with the following interesting

Theorem 5.16. The correspondence

$$
(a \otimes 1) \delta(b) \longmapsto \delta(a)(b \otimes 1),
$$

where $a, b \in A$, extends to a closed linear mapping acting on $A \otimes A$.

Proof. The statement follows immediately from the formula

$$
(x \otimes z|(a \otimes 1) \delta(b)| W(y \otimes u))=\left(W\left(x \otimes \widehat{J} Q^{-1} u\right)|\delta(a)(b \otimes 1)| y \otimes \widehat{J} Q z\right),
$$

that holds for all $a, b \in A, x, y \in \mathcal{H}, z \in \mathcal{D}(Q)$ and $u \in \mathcal{D}\left(Q^{-1}\right)$ (the reader should notice that each of the vectors $x \otimes z, W(y \otimes u), W\left(x \otimes \widehat{J} Q^{-1} u\right)$ and $y \otimes \widehat{J} Q z$ run over a linearly dense subset of $\mathcal{H} \otimes \mathcal{H})$. 
To prove (5.37) we start with the formula (3.14) proving the manageability of the Kac-Takesaki operator. The right hand side may be rewritten in the following way:

$$
\begin{aligned}
(x \otimes z \mid W(y \otimes u)) & =\left(J y \otimes Q z \mid W^{*}\left(J x \otimes Q^{-1} u\right)\right) \\
& =\left((J \otimes \widehat{J})(y \otimes \widehat{J} Q z) \mid W^{*}\left(J x \otimes Q^{-1} u\right)\right) \\
& =\left((J \otimes \widehat{J}) W^{*}\left(J x \otimes Q^{-1} u\right) \mid y \otimes \widehat{J} Q z\right) \\
& =\left(W(J \otimes \widehat{J})\left(J x \otimes Q^{-1} u\right) \mid y \otimes \widehat{J} Q z\right) \\
& =\left(x \otimes \widehat{J} Q^{-1} u \mid W^{*}(y \otimes \widehat{J} Q z)\right),
\end{aligned}
$$

where in the fourth step we used (5.22). Replacing in the above formula $x$ by $a^{*} x$ and $y$ by by we obtain

$$
(x \otimes z|(a \otimes 1) W(b \otimes 1)| y \otimes u)=\left(x \otimes \widehat{J} Q^{-1} u\left|(a \otimes 1) W^{*}(b \otimes 1)\right| y \otimes \widehat{J} Q z\right)
$$

and (5.37) follows (cf. (3.7)).

\section{A GNS-Mapping on $\widehat{A}$}

One of the aim of this paper is to show that $(\widehat{A}, \widehat{\delta})$ is a weighted Hopf $C^{*}$-algebra. To this end we have to introduce a right invariant weight $\widehat{h}$ on $\widehat{A}$. It will be related to a GNS-mapping $\widehat{\eta}: \widehat{A} \rightarrow \mathcal{H}$. However the definition of $\widehat{\eta}$ is not simple. We start with the following technical result, in which the commutant $\eta^{\prime}$ of the GNS mapping $\eta$ is used.

Proposition 6.1. Let $z \in \mathcal{D}(\widehat{F})$. Then for any $a, b \in \mathcal{D}\left(\eta^{\prime}\right)$ and $x, y \in \mathcal{H}$ we have :

$$
\left(z \otimes y \mid W\left(a^{*} x \otimes \eta^{\prime}(b)\right)\right)=\left(b^{*} y \otimes \eta^{\prime}(a) \mid W^{*}(\widehat{F} z \otimes x)\right) .
$$

Proof. We may assume that $z=\eta^{\prime \prime}(c)$ for some $c \in \mathcal{D}_{0}$. Then $\widehat{F} z=\eta^{\prime \prime}\left(\kappa^{M}(c)^{*}\right)$ by Proposition 4.3. Furthermore, we may assume that $x, y \in \mathcal{D}(Q)$ and $\eta^{\prime}(a), \eta^{\prime}(b) \in \mathcal{D}\left(Q^{-1}\right)$. For any $d \in A$ we set

$$
\begin{array}{ll}
\varphi_{1}(d)=\left(x \mid d \eta^{\prime}(a)\right), & \varphi_{2}(d)=\left(Q^{-1} \eta^{\prime}(a) \mid R(d) Q x\right) \\
\psi_{1}(d)=\left(y \mid d \eta^{\prime}(b)\right), & \psi_{2}(d)=\left(Q^{-1} \eta^{\prime}(b) \mid R(d) Q y\right) .
\end{array}
$$

Then $\varphi_{1}, \varphi_{2}, \psi_{1}, \psi_{2} \in A_{*}, \varphi_{2}=\varphi_{1}^{*} \kappa, \psi_{2}=\psi_{1}^{*} \kappa$ and hence by (1.9) $W_{\varphi_{1}}^{*}=W_{\varphi_{2}}$ and $W_{\psi_{1}}^{*}=W_{\psi_{2}}$. With this notation, the left hand side of (6.1)

$$
\begin{aligned}
L H S & =\left(\eta^{\prime \prime}(c) \mid W_{\psi_{1}} a^{*} x\right)=\left(W_{\psi_{2}} \eta^{\prime \prime}(c) \mid a^{*} x\right)=\left(\eta^{\prime \prime}\left(\psi_{2} * c\right) \mid a^{*} x\right) \\
& =\left(a \eta^{\prime \prime}\left(\psi_{2} * c\right) \mid x\right)=\left(\left(\psi_{2} * c\right) \eta^{\prime}(a) \mid x\right)=\overline{\varphi_{1}\left(\psi_{2} * c\right)} \\
& =\overline{\left(\varphi_{1} * \psi_{2}\right)(c)},
\end{aligned}
$$

where $\varphi_{1} * \psi_{2} \in A_{*}$. Replacing in the above computation $x, y, a, b, c$ by $y, x, b, a, \kappa^{M}(c)^{*}$ respectively, we see that $\psi_{1} * \varphi_{2} \in A_{*}$ and the complex conjugate of the right hand side of (6.1) equals $\overline{\left(\psi_{1} * \varphi_{2}\right)\left(\kappa^{M}(c)^{*}\right)}$. For the proof of (6.1) it remains to show that

$$
\left(\psi_{1} * \varphi_{2}\right)\left(\kappa^{M}(c)^{*}\right)=\overline{\left(\varphi_{1} * \psi_{2}\right)(c)} \text {. }
$$

Since

$$
\left(W_{\psi_{1} * \varphi_{2}}\right)^{*}=\left(W_{\psi_{1}} W_{\varphi_{2}}\right)^{*}=W_{\varphi_{2}}^{*} W_{\psi_{1}}^{*}=W_{\varphi_{1}} W_{\psi_{2}}=W_{\varphi_{1} * \psi_{2}}
$$

we have $\varphi_{1} * \psi_{2}=\left(\psi_{1} * \varphi_{2}\right)^{*} \kappa$ by Proposition [2.9] Hence $\left.\left(\psi_{1} * \varphi_{2}\right)^{*} \kappa^{M}\right|_{A}=\left(\psi_{1} * \varphi_{2}\right)^{*}{ }^{\circ} \kappa$ is $\sigma$-weakly continuous. It shows that

$$
\begin{aligned}
\left(\psi_{1} * \varphi_{2}\right)\left(\kappa^{M}(c)^{*}\right) & =\overline{\left(\psi_{1} * \varphi_{2}\right)^{*}\left(\kappa^{M}(c)\right)}=\overline{\left(\left(\psi_{1} * \varphi_{2}\right)^{*} \circ \kappa^{M}\right)(c)} \\
& =\overline{\left(\psi_{1} * \varphi_{2}\right)^{*} \kappa(c)}=\overline{\left(\varphi_{1} * \psi_{2}\right)(c)}
\end{aligned}
$$

and (6.1) follows.

Relation (6.1) may be rewritten in the following way :

It shows that

$$
\left(y \mid W\left(z, a^{*} x\right) \eta^{\prime}(b)\right)=\left(b^{*} y \mid W_{\omega_{x, \eta^{\prime}(a)}^{*}}^{*} \widehat{F} z\right)
$$

$$
W\left(z, a^{*} x\right) \eta^{\prime}(b)=b W_{\omega_{x, \eta^{\prime}(a)}^{*}} \widehat{F} z
$$


This relation holds for all $b \in \mathcal{D}\left(\eta^{\prime}\right)$. Therefore

$$
W\left(z, a^{*} x\right) \in \mathcal{D}\left(\eta^{\prime \prime}\right) \quad \text { and } \quad \eta^{\prime \prime}\left(W\left(z, a^{*} x\right)\right)=W_{\omega_{x, \eta^{\prime}(a)}^{*}} \widehat{F} z .
$$

Remembering that $W\left(z, a^{*} x\right) \in A$ and $\left.\eta^{\prime \prime}\right|_{A}=\eta$, we obtain

Proposition 6.2. Let $z \in \mathcal{D}(\widehat{F}), a \in \mathcal{D}\left(\eta^{\prime}\right)$ and $x \in \mathcal{H}$. Then $W\left(z, a^{*} x\right) \in \mathcal{D}(\eta)$ and

$$
\eta\left(W\left(z, a^{*} x\right)\right)=W_{\omega_{x, \eta^{\prime}(a)}^{*}} \widehat{F} z .
$$

It should be remarked that the vectors on the right hand side form a dense subset of $\mathcal{H}$. Indeed, the range of $\widehat{F}$ is dense in $\mathcal{H}$, the set of operators $W_{\omega_{x, \eta^{\prime}(a)}^{*}}^{*}$ is dense in $\widehat{A}$ and $\widehat{A}$ is a non degenerate $C^{*}$-algebra acting on $\mathcal{H}$.

Let $\Phi$ be the set :

$$
\Phi=\{(x, y) \in \mathcal{H} \times \mathcal{H}: W(x, y) \in \mathcal{D}(\eta)\} .
$$

By Proposition 6.2 and the above remark, $\Phi$ is dense in $\mathcal{H} \times \mathcal{H}$ and $\{\eta(W(x, y)):(x, y) \in \Phi\}$ is dense in $\mathcal{H}$. Therefore the relation

$$
(y \mid a x)=(\eta(W(x, y)) \mid z) \quad \text { for all } \quad(x, y) \in \Phi .
$$

gives a one to one correspondence between elements $a \in \widehat{A}$ and vectors $z \in \mathcal{H}$. This correspondence will be denoted by $\widehat{\eta}$ : we write

$$
z=\widehat{\eta}(a)
$$

if the relation (6.2) holds. Clearly $\widehat{\eta}$ is a linear mapping from $\widehat{A}$ to $\mathcal{H}$ and the domain $\mathcal{D}(\widehat{\eta})$ consists of all elements $a \in \widehat{A}$ such that there exists $z \in \mathcal{H}$ satisfying (6.2).

Let $\varphi \in A^{*}$. We say that $\varphi$ is $L^{2}$-bounded if there exists $z_{\varphi} \in \mathcal{H}$ such that

$$
\varphi(a)=\left(z_{\varphi} \mid \eta(a)\right)
$$

for all $a \in \mathcal{D}(\eta)$.

Proposition 6.3. Let $\varphi \in A^{*}$ be an $L^{2}$-bounded functional. Then $\varphi$ is $\sigma$-weakly continuous.

Proof. At first we notice that for any $b \in \mathcal{D}(\eta)$ the functional $b \varphi$ is $\sigma$-weakly continuous. Indeed for any $a \in A, a b \in \mathcal{D}(\eta)$ and

$$
(b \varphi)(a)=\varphi(a b)=\left(z_{\varphi} \mid \eta(a b)\right)=\left(z_{\varphi} \mid a \eta(b)\right) .
$$

The set of $\sigma$-weakly continuous functionals is norm closed. Inserting $b=e_{\alpha}$ (where $\left\{e_{\alpha}\right\}$ is an approximate identity for $A$ ) and using Appendix A we see that $\varphi$ is $\sigma$-weakly continuous.

Remark that the set of all $L^{2}$-bounded functionals is norm dense in $A_{*}$ (cf. the proof of Assertion 1 of Proposition 6.5).

Theorem 6.4. (1) The domain $\mathcal{D}(\widehat{\eta})$ is dense in $\widehat{A}$ and the range of $\widehat{\eta}$ is dense in $\mathcal{H}$.

(2) The set $\mathcal{D}(\widehat{\eta})$ is a left ideal in $\widehat{A}$ and $\widehat{\eta}(b a)=b \widehat{\eta}(a)$ for all $b \in \widehat{A}$ and $a \in \mathcal{D}(\widehat{\eta})$.

(3) The mapping $\widehat{\eta}$ of $\widehat{A}$ to $\mathcal{H}$ is injective and closed with respect to the strong topology on $\widehat{A}$ and the norm topology on $\mathcal{H}$.

(4) Let $\varphi$ be an $L^{2}$-bounded functional on $A$. Then $W_{\varphi}^{*} \in \mathcal{D}(\widehat{\eta})$ and $\widehat{\eta}\left(W_{\varphi}^{*}\right)=z_{\varphi}$.

Proof.

Ad 4. Using Proposition 6.3 we see that $\varphi \in A_{*}$ and hence $W_{\varphi} \in \widehat{A}$. For any $(x, y) \in \Phi$ we have

$$
\begin{aligned}
\left(y \mid W_{\varphi}^{*} x\right) & =\overline{\left(x \mid W_{\varphi} y\right)}=\overline{\varphi(W(x, y))} \\
& =\overline{\left(z_{\varphi} \mid \eta(W(x, y))\right)}=\left(\eta(W(x, y)) \mid z_{\varphi}\right) .
\end{aligned}
$$

It shows that $W_{\varphi}^{*} \in \mathcal{D}(\widehat{\eta})$ and $\widehat{\eta}\left(W_{\varphi}^{*}\right)=z_{\varphi}$.

Ad 1. Now, let $y \in \mathcal{H}, c \in \mathcal{D}\left(\eta^{\prime}\right)$ and

$$
\varphi(b)=\left(y \mid b \eta^{\prime}(c)\right) \quad \text { for } \quad b \in A .
$$

Then $\varphi \in A_{*}$ and for any $b \in \mathcal{D}(\eta), \varphi(b)=\left(c^{*} y \mid \eta(b)\right)$. By Assertion $4, W_{\varphi}^{*} \in \mathcal{D}(\widehat{\eta})$ and $\widehat{\eta}\left(W_{\varphi}^{*}\right)=$ $c^{*} y$. The set of functionals $\varphi$ of the form (6.3) is linearly dense in $A_{*}$. Therefore $\mathcal{D}(\widehat{\eta})$ is dense in $\widehat{A}$. Moreover, the set of vectors of the form $c^{*} y$ (where $c \in \mathcal{D}\left(\eta^{\prime}\right)$ and $y \in \mathcal{H}$ ) is dense in $\mathcal{H}$. So is the range of $\widehat{\eta}$. 
Ad 2. Let $(x, y) \in \Phi$ and $\varphi \in A_{*}$. Then for any $\psi \in A^{*}$ we have

$$
\begin{aligned}
\psi(\varphi * W(x, y)) & =(\psi * \varphi)(W(x, y))=\left(x \mid W_{\psi * \varphi} y\right) \\
& =\left(x \mid W_{\psi} W_{\varphi} y\right)=\psi\left(W\left(x, W_{\varphi} y\right)\right) .
\end{aligned}
$$

It shows that $\varphi * W(x, y)=W\left(x, W_{\varphi} y\right)$. Since $W(x, y) \in \mathcal{D}(\eta)$, we see that $W\left(x, W_{\varphi} y\right) \in \mathcal{D}(\eta)$. Hence $\left(x, W_{\varphi} y\right) \in \Phi$. We also have

$$
\eta\left(W\left(x, W_{\varphi} y\right)\right)=W_{\varphi} \eta(W(x, y)) .
$$

Let $a \in \mathcal{D}(\widehat{\eta})$. Then for any $(x, y) \in \Phi$ we have $(\eta(W(x, y)) \mid \widehat{\eta}(a))=(y \mid a x)$. Replacing $(x, y)$ by $\left(x, W_{\varphi} y\right)$ we obtain

$$
\left(W_{\varphi} \eta(W(x, y)) \mid \widehat{\eta}(a)\right)=\left(W_{\varphi} y \mid a x\right) .
$$

Therefore $\left(\eta(W(x, y)) \mid W_{\varphi}^{*} \widehat{\eta}(a)\right)=\left(y \mid W_{\varphi}^{*} a x\right)$. It shows that $W_{\varphi}^{*} a \in \mathcal{D}(\widehat{\eta})$ and $\widehat{\eta}\left(W_{\varphi}^{*} a\right)=W_{\varphi}^{*} \widehat{\eta}(a)$. Remembering that $\left\{W_{\varphi}^{*}: \varphi \in A_{*}\right\}=\left\{(\operatorname{id} \otimes \varphi)\left(W^{*}\right): \varphi \in \mathcal{L}(\mathcal{H})_{*}\right\}$ is dense in $\widehat{A}$ (cf. (3.16)), we obtain Assertion 2 in full generality.

Ad 3. It follows immediately from (6.2).

In this way we have shown that $\widehat{\eta}: \widehat{A} \rightarrow \mathcal{H}$ is a closed, densely defined GNS-mapping. In what follows $\widehat{\eta}^{\prime}: \widehat{A}^{\prime} \rightarrow \mathcal{H}$ will denote the commutant of $\widehat{\eta}$.

If $\varphi \in A^{*}$ is $L^{2}$-bounded, then according to Assertion $4, W_{\varphi}^{*} \in \mathcal{D}(\widehat{\eta})$ and $\varphi(b)=\left(\widehat{\eta}\left(W_{\varphi}^{*}\right) \mid \eta(b)\right)$ for any $b \in \mathcal{D}(\eta)$. Remembering that $\varphi$ is $\sigma$-weakly continuous (the same letter will denote its $\sigma$-weakly continuous extension to $\left.A^{\prime \prime}\right)$ and that $\eta^{\prime \prime}$ is the extension of $\eta$ with $\mathcal{D}(\eta)$ being a core for $\eta^{\prime \prime}$ (cf. Theorem B.2), we obtain

$$
\varphi(b)=\left(\widehat{\eta}\left(W_{\varphi}^{*}\right) \mid \eta^{\prime \prime}(b)\right)
$$

for any $b \in \mathcal{D}\left(\eta^{\prime \prime}\right)$. Let

$$
\widehat{A}_{0}=\left\{W_{\varphi}^{*}: \varphi, \varphi^{*} \text { o } \in A^{*} \text { and } \varphi, \varphi^{*} \kappa \text { are } L^{2} \text {-bounded }\right\}
$$

and

$$
\widehat{A_{1}}=\left\{W_{\varphi}^{*}: \varphi, \varphi^{*} \circ \kappa \in A^{*} \text { and } \varphi \text { is } L^{2} \text {-bounded }\right\} .
$$

Proposition 6.5. $\quad$ (1) $\widehat{A}_{0}$ is a norm dense linear subset of $\widehat{A}$.

(2) $\widehat{A}_{0}$ is a $*$-subalgebra of $\mathcal{L}(\mathcal{H})$.

(3) $\widehat{A}_{0} \subset \mathcal{D}(\widehat{\eta})$ and $\widehat{\eta}\left(\widehat{A}_{0}\right)$ is dense in $\mathcal{H}$.

(4) If $a \in \widehat{A}_{0}$, then $\widehat{\eta}(a) \in \mathcal{D}\left(\widehat{F}^{*}\right)$ and $\widehat{F}^{*} \widehat{\eta}(a)=\widehat{\eta}\left(a^{*}\right)$.

Proof. Clearly $\widehat{A}_{0} \subset \widehat{A}_{1}$. Assume that $\varphi, \psi$ are $L^{2}$-bounded functionals in $A^{*}$ such that $\varphi^{*} \circ$, $\psi^{*} \circ \in A^{*}$. Then using (1.7) and (1.9) we obtain

$$
\begin{gathered}
W_{\varphi} W_{\psi}^{*}=\left(W_{\psi} W_{\varphi^{*} \mathrm{o}_{\kappa}}\right)^{*}=W_{\psi *\left(\varphi^{*} \mathrm{o}_{\kappa}\right)}^{*}, \\
W_{\varphi} W_{\psi}^{*}=W_{\varphi} W_{\psi^{*} \mathrm{o}_{\kappa}}=W_{\varphi *\left(\psi^{*} \mathrm{o}_{\kappa}\right)} .
\end{gathered}
$$

Let $\rho=\psi *\left(\varphi^{*} \circ \kappa\right)$. Using Proposition [2.9] we see that $\rho^{*} \kappa=\varphi *\left(\psi^{*} \circ \kappa\right) \in A^{*}$. We shall prove that $\rho$ and $\rho^{*} \circ \kappa$ are $L^{2}$-bounded. Let $a \in \mathcal{D}(\eta)$. Then (cf. 1.5) $\left(\varphi^{*} \circ \kappa\right) * a \in \mathcal{D}(\eta)$ and

$$
\begin{aligned}
\rho(a) & =\left(\psi *\left(\varphi^{*} \circ \kappa\right)\right)(a)=\psi\left(\left(\varphi^{*} \circ \kappa\right) * a\right)=\left(z_{\psi} \mid \eta\left(\left(\varphi^{*} \circ \kappa\right) * a\right)\right) \\
& =\left(z_{\psi} \mid W_{\varphi^{* \circ} \kappa} \eta(a)\right)=\left(W_{\varphi} z_{\psi} \mid \eta(a)\right) .
\end{aligned}
$$

Hence $\rho$ is $L^{2}$-bounded. Exchanging the role of $\varphi$ and $\psi$ in the above argument, we see that $\rho^{*}$ o $\kappa$ is $L^{2}$-bounded. Hence $W_{\varphi} W_{\psi}^{*}=W_{\rho}^{*} \in \widehat{A}_{0}$. In this way we showed that

$$
\widehat{A}_{1}^{*} \widehat{A}_{1} \subset \widehat{A}_{0} \subset \widehat{A}_{1} .
$$

Ad 1. Let $y \in \mathcal{D}(Q)$ and $c \in \mathcal{D}\left(\eta^{\prime}\right)$ such that $\eta^{\prime}(c) \in \mathcal{D}\left(Q^{-1}\right)$ and $\varphi=\omega_{y, \eta^{\prime}(c)}$. Then for any $a \in \mathcal{D}(\eta)$ we have

$$
\varphi(a)=\left(y \mid a \eta^{\prime}(c)\right)=(y \mid c \eta(a))=\left(c^{*} y \mid \eta(a)\right) .
$$

It shows that $\varphi$ is $L^{2}$-bounded. On the other hand, $\varphi^{*}=\omega_{\eta^{\prime}(c), y}$ and

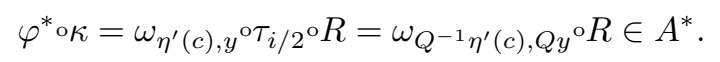


Therefore $W_{\omega_{y, \eta^{\prime}(c)}^{*}} \in \widehat{A}_{1}$. One can easily check that the set of functionals $\omega_{\eta^{\prime}(b), \eta^{\prime}(c)}$ (where $b$ and $c$ satisfy the above condition) is linearly dense in $\mathcal{L}(\mathcal{H})_{*}$ by Proposition 4.2 Hence $\widehat{A}_{1}$ is dense in $\widehat{A}$ (cf. (3.16)). The first inclusion in (6.7) shows now that $\widehat{A}_{0}$ is dense in $\widehat{A}$.

Ad 2. If $W_{\varphi}^{*} \in \widehat{A}_{0}$, then $W_{\varphi^{*} \mathrm{o}_{\kappa}}^{*} \in \widehat{A}_{0}$ and $W_{\varphi}=W_{\varphi^{*} \kappa}^{*}$ by (1.9). Hence $\widehat{A}_{0}$ is invariant under the involution. Formula (6.7) shows now that $\widehat{A}_{0}$ is a subalgebra.

Ad 3. Statement 4 of Theorem $\left[6.4\right.$ shows that $\widehat{A}_{1} \subset \mathcal{D}(\widehat{\eta})$. Let $y, c$ and $\varphi$ be as in the proof of Assertion 1. Using Assertion 4 of Theorem 6.4 and formula (6.8) we see that

$$
\widehat{\eta}\left(W_{\omega_{y, \eta^{\prime}(c)}^{*}}^{*}\right)=c^{*} y .
$$

Using Proposition 4.2 we find that $\widehat{\eta}\left(\widehat{A}_{1}\right)$ is dense in $\mathcal{H}$. So is $\widehat{\eta}\left(\widehat{A}_{0}\right)$; by [6.7), $\widehat{\eta}\left(\widehat{A}_{0}\right)$ contains $\widehat{\eta}\left(\widehat{A}_{1}^{*} \widehat{A}_{1}\right)=\widehat{A}_{1}^{*} \widehat{\eta}\left(\widehat{A}_{1}\right)$.

Ad 4. If $a \in \widehat{A}_{0}$, then $a=W_{\varphi}^{*}$ and $a^{*}=W_{\psi}^{*}$, where $\varphi, \psi$ are bounded and $L^{2}$-bounded functionals on $A$ such that $\psi=\varphi^{*}$ o . Remembering that $\psi$ and $\varphi^{*}$ are $\sigma$-weakly continuous and that $\kappa^{M}$ is a closure of $\kappa$, we see that $\psi(b)=\varphi^{*} \kappa^{M}(b)$ for any $b \in \mathcal{D}\left(\kappa^{M}\right)$.

Let $b \in \mathcal{D}_{0}$ (cf. (5.2)). Then $b, \kappa^{M}(b)^{*} \in \mathcal{D}\left(\eta^{\prime \prime}\right)$ and using (6.4), we have

$$
\begin{aligned}
\left(\eta^{\prime \prime}\left(\kappa^{M}(b)^{*}\right) \mid \widehat{\eta}(a)\right) & =\overline{\varphi\left(\kappa^{M}(b)^{*}\right)}=\varphi^{*}\left(\kappa^{M}(b)\right) \\
& =\psi(b)=\left(\widehat{\eta}\left(a^{*}\right) \mid \eta^{\prime \prime}(b)\right) .
\end{aligned}
$$

Since $\left\{\eta^{\prime \prime}(b): b \in \mathcal{D}_{0}\right\}$ is a core for $\widehat{F}$ and $\widehat{F} \eta^{\prime \prime}(b)=\eta^{\prime \prime}\left(\kappa^{M}(b)^{*}\right)$, we obtain $\widehat{\eta}(a) \in \mathcal{D}\left(\widehat{F}^{*}\right)$ and $\widehat{F}^{*} \widehat{\eta}(a)=\widehat{\eta}\left(a^{*}\right)$.

Remark 6.6. To show that $\widehat{A}_{0} \mathcal{H}$ is dense in $\mathcal{H}$ we suppose that $x \in \mathcal{H}$ is orthogonal to $W_{\omega_{\eta^{\prime}(b), \eta^{\prime}(c)}^{*}} \mathcal{H}$ for all $b, c \in \mathcal{D}\left(\eta^{\prime}\right)$ with $\eta^{\prime}(b) \in \mathcal{D}(Q)$ and $\eta^{\prime}(c) \in \mathcal{D}\left(Q^{-1}\right)$. Then

$$
\left(\eta^{\prime}(b) \mid W(y, x) \eta^{\prime}(c)\right)=\left(W_{\omega_{\eta^{\prime}(b), \eta^{\prime}(c)}^{*}}^{*} y \mid x\right)=0
$$

and hence $W(y, x)=0$ for all $y \in \mathcal{H}$. Using Proposition 3.2 we see that

$$
\|x\|^{2} 1=\sum_{n \in \mathbb{N}} W\left(e_{n}, x\right)^{*} W\left(e_{n}, x\right)=0
$$

and $x=0$. Thus $\widehat{A}_{0} \mathcal{H}$ is dense in $\mathcal{H}$ and hence $\widehat{A}_{0} \widehat{\eta}\left(\widehat{A}_{0}\right)$ is dense in $\widehat{\eta}\left(\widehat{A}_{0}\right)$.

Proposition 6.7. Let $\widehat{a} \in \mathcal{L}(\mathcal{H})$ and $z \in \mathcal{H}$. Then the following three conditions are equivalent:

(1) $\widehat{a} \in \mathcal{D}\left(\widehat{\eta}^{\prime}\right)$ and $\widehat{\eta}^{\prime}(\widehat{a})=z$.

(2) For any $\widehat{b} \in \widehat{A}_{0}$,

$$
\widehat{b} z=\widehat{a} \widehat{\eta}(\widehat{b}) .
$$

(3) $(z, x) \in \Phi$ and $\eta(W(z, x))=\widehat{a}^{*} x$ for all $x \in \mathcal{H}$.

Proof. Ad $1 \Rightarrow 2$. It is clear.

Ad $2 \Rightarrow 3$. Let $y, c$ and $\varphi$ be as in the proof of Assertion 1 of Proposition 6.5 Then $W_{\varphi}^{*} \in \widehat{A}$. Moreover, $\varphi(a)=\left(c^{*} y \mid \eta(a)\right)$ for all $a \in \mathcal{D}(\eta)$ and $\widehat{\eta}\left(W_{\varphi}^{*}\right)=c^{*} y$ by (6.9). Replacing $\widehat{b}$ by $W_{\varphi}^{*}$ in (6.10), we obtain

$$
W_{\varphi}^{*} z=\widehat{a} c^{*} y
$$

Let $x \in \mathcal{H}$. Then

$$
\left(y \mid W(z, x) \eta^{\prime}(c)\right)=\varphi(W(z, x))=\left(W_{\varphi}^{*} z \mid x\right)=\left(\widehat{a} c^{*} y \mid x\right)=\left(y \mid c \widehat{a}^{*} x\right)
$$

for all $y \in \mathcal{D}(Q)$. Therefore $W(z, x) \eta^{\prime}(c)=c \widehat{a}^{*} x$ for any $c \in \mathcal{D}\left(\eta^{\prime}\right)$ such that $\eta^{\prime}(c) \in \mathcal{D}\left(Q^{-1}\right)$. Using Proposition 4.2, we see that $W(z, x) \in \mathcal{D}\left(\eta^{\prime \prime}\right)$ (hence $\left.(z, x) \in \Phi\right)$ and $\eta^{\prime \prime}(W(z, x))=\widehat{a}^{*} x$. Since $W(z, x) \in A$ and $\eta^{\prime \prime} \mid A=\eta$, we see that $W(z, x) \in \mathcal{D}(\eta)$ and $\eta(W(z, x))=\widehat{a}^{*} x$ for all $x \in \mathcal{H}$.

Ad $3 \Rightarrow 1$. Assume that $(z, x) \in \Phi$ and $\eta(W(z, x))=\widehat{a}^{*} x$ for all $x \in \mathcal{H}$. Let $\widehat{b} \in \mathcal{D}(\widehat{\eta})$. Then

$$
(x \mid \widehat{a} \widehat{\eta}(\widehat{b}))=\left(\widehat{a}^{*} x \mid \widehat{\eta}(\widehat{b})\right)=(\eta(W(z, x)) \mid \widehat{\eta}(\widehat{b}))=(x \mid \widehat{b} z),
$$

and hence we have $\widehat{b} z=\widehat{a} \widehat{\eta}(\widehat{b})$ for all $\widehat{b} \in \mathcal{D}(\widehat{\eta})$. Thus $\widehat{a} \in \widehat{A}^{\prime}, \widehat{a} \in \mathcal{D}\left(\widehat{\eta}^{\prime}\right)$ and $\widehat{\eta}^{\prime}(\widehat{a})=z$.

Corollary 6.8. The set $\widehat{A}_{0}$ is a core for $\widehat{\eta}$ (resp. $\widehat{\eta}^{\prime \prime}$ ) with respect to the strong topology in $\widehat{A}$ (resp. $\widehat{A}^{\prime \prime}$ ) and the norm topology in $\mathcal{H}$. 
Proof. Let $\widehat{\eta}_{0}=\left.\widehat{\eta}\right|_{\widehat{A}_{0}}$. By Proposition 6.7 (equivalence of the first two conditions), the commutant of $\widehat{\eta}_{0}$ coincides with $\widehat{\eta}^{\prime}$. Therefore $\widehat{\eta}_{0}^{\prime \prime}=\widehat{\eta}^{\prime \prime}$. By Theorem B.2 $\widehat{A}_{0}=\mathcal{D}\left(\widehat{\eta}_{0}\right)$ is a core for $\widehat{\eta}^{\prime \prime}$. Consequently it is a core for $\widehat{\eta}$.

Now we shall show that the operator $\widehat{\Delta}$ introduced in (5.1) is the dual modular operator. Let $a, b \in \mathcal{D}\left(\widehat{\eta}^{\prime}\right)$. By Proposition $6.7 W\left(\widehat{\eta}^{\prime}(a), \widehat{\eta}^{\prime}(b)\right) \in \mathcal{D}(\eta)$ and

$$
\eta\left(W\left(\widehat{\eta}^{\prime}(a), \widehat{\eta}^{\prime}(b)\right)\right)=a^{*} \widehat{\eta}^{\prime}(b) .
$$

Exchanging $a$ and $b$, we see that $W\left(\widehat{\eta}^{\prime}(b), \widehat{\eta}^{\prime}(a)\right) \in \mathcal{D}(\eta)$ and $\eta\left(W\left(\widehat{\eta}^{\prime}(b), \widehat{\eta}^{\prime}(a)\right)\right)=b^{*} \widehat{\eta}^{\prime}(a)$. Moreover, by Proposition 2.4 $W\left(\widehat{\eta}^{\prime}(a), \widehat{\eta}^{\prime}(b)\right) \in \mathcal{D}(\kappa)$ and $\kappa\left(W\left(\widehat{\eta}^{\prime}(a), \widehat{\eta}^{\prime}(b)\right)\right)^{*}=W\left(\widehat{\eta}^{\prime}(b), \widehat{\eta}^{\prime}(a)\right)$. Let $\mathcal{D}_{0}$ be the subset of $A^{\prime \prime}$ introduced in (5.2). Then $W\left(\widehat{\eta}^{\prime}(a), \widehat{\eta}^{\prime}(b)\right) \in \mathcal{D}_{0}$, and using (5.19), we see that $a^{*} \widehat{\eta}^{\prime}(b) \in \mathcal{D}(\widehat{F})$ and

$$
\widehat{F} a^{*} \widehat{\eta}^{\prime}(b)=b^{*} \widehat{\eta}^{\prime}(a)
$$

We shall use the Tomita-Takesaki theory for the von Neumann algebra $\widehat{A}^{\prime \prime}$ and the GNS-mapping $\widehat{\eta}^{\prime \prime}$. In particular, $\widehat{S}_{T}$ and $\widehat{F}_{T}$ (the subscript $T$ refers to Tomita-Takesaki) will denote the left and the right involution operators, respectively. By definition, the linear span of $\left\{\widehat{b}^{*} \widehat{\eta}(\widehat{a}): \widehat{a}, \widehat{b} \in \mathcal{D}(\widehat{\eta})\right\}$ is a core for $\widehat{S}_{T}$ and

$$
\widehat{S}_{T} \widehat{b}^{*} \widehat{\eta}(\widehat{a})=\widehat{a}^{*} \widehat{\eta}(\widehat{b})
$$

Similarly the linear span of $\left\{b^{*} \widehat{\eta}^{\prime}(a): a, b \in \mathcal{D}\left(\widehat{\eta}^{\prime}\right)\right\}$ is a core for $\widehat{F}_{T}$ and

$$
\widehat{F}_{T} b^{*} \hat{\eta}^{\prime}(a)=a^{*} \hat{\eta}^{\prime}(b) \text {. }
$$

It is known that $\widehat{F}_{T}=\widehat{S}_{T}^{*}$. Formula (6.11) shows that $\widehat{F}_{T} \subset \widehat{F}$.

Theorem 6.9. The operator $\widehat{F}$ introduced by (5.19) coincides with $\widehat{F}_{T}$.

Proof. We have to show that $\widehat{F} \subset \widehat{F}_{T}$. Let $x \in \mathcal{D}(\widehat{F})$. Let us consider linear operators

$$
\begin{gathered}
T: \widehat{\eta}(a) \longmapsto a x, \\
T^{b}: \widehat{\eta}(b) \longmapsto b \widehat{F} x,
\end{gathered}
$$

where $a$ and $b$ run over $\widehat{A}_{0}$. By Statement 3 of Proposition 6.5 the domains $\mathcal{D}(T)=\mathcal{D}\left(T^{b}\right)$ are dense in $\mathcal{H}$.

According to Assertion 2 of Proposition $6.5 a^{*} b, b^{*} a \in \widehat{A}_{0}$ for any $a, b \in \widehat{A}_{0}$. Using Assertion 4 of the same proposition, we have

$$
\begin{aligned}
(\widehat{\eta}(b) \mid T \widehat{\eta}(a)) & =(\widehat{\eta}(b) \mid a x)=\left(\widehat{\eta}\left(a^{*} b\right) \mid x\right)=\left(\widehat{F}^{*} \widehat{\eta}\left(b^{*} a\right) \mid x\right) \\
& =\left(\widehat{F} x \mid \widehat{\eta}\left(b^{*} a\right)\right)=(b \widehat{F} x \mid \widehat{\eta}(a))=\left(T^{b} \widehat{\eta}(b) \mid \widehat{\eta}(a)\right) .
\end{aligned}
$$

It shows that $T^{b} \subset T^{*}$. Therefore $T^{*}$ is densely defined and $T$ is closable. In what follows $T$ will denote the closure of the first operator in (6.14). Replacing $a$ by $b^{*} a$ in (6.14), we obtain

$$
T b^{*} \widehat{\eta}(a)=T \widehat{\eta}\left(b^{*} a\right)=b^{*} a x=b^{*} T \widehat{\eta}(a)
$$

for any $a, b \in \widehat{A}_{0}$. It shows that $b^{*} T \subset T b^{*}$. Using this formula, one can easily show that $T$ is affiliated with the von Neumann algebra $\widehat{A}^{\prime}$.

Let $f$ be a continuous function on $\mathbb{R}_{+}$such that $\left\{t f\left(t^{2}\right): t \in \mathbb{R}_{+}\right\}$is bounded, and $T_{f}=$ $T f\left(T^{*} T\right)$. Then $T_{f} \in \widehat{A}^{\prime}, T_{f} y=f\left(T T^{*}\right) T y$ for any $y \in \mathcal{D}(T)$, and $T_{f}^{*} z=f\left(T^{*} T\right) T^{*} z$ for any $z \in \mathcal{D}\left(T^{*}\right)$. Replacing $y$ by $\widehat{\eta}(a)$, we obtain

$$
T_{f} \widehat{\eta}(a)=f\left(T T^{*}\right) a x=a f\left(T T^{*}\right) x .
$$

This formula holds for all $a \in \widehat{A}_{0}$. Then Proposition 6.7 shows now that $T_{f} \in \mathcal{D}\left(\widehat{\eta}^{\prime}\right)$ and $\widehat{\eta}^{\prime}\left(T_{f}\right)=$ $f\left(T T^{*}\right) x$. Similarly, replacing $z$ by $\widehat{\eta}(b)$, we obtain

$$
T_{f}^{*} \widehat{\eta}(b)=f\left(T^{*} T\right) b \widehat{F} x=b f\left(T^{*} T\right) \widehat{F} x .
$$

This formula holds for all $b \in \widehat{A}_{0}$. Then Proposition 6.7 shows now that $T_{f}^{*} \in \mathcal{D}\left(\widehat{\eta}^{\prime}\right)$ and $\widehat{\eta}^{\prime}\left(T_{f}^{*}\right)=$ $f\left(T^{*} T\right) \widehat{F} x$. Combining the two results, we see that $f\left(T T^{*}\right) x \in \mathcal{D}\left(\widehat{F}_{T}\right)$ and

$$
\widehat{F}_{T} f\left(T T^{*}\right) x=f\left(T^{*} T\right) \widehat{F} x .
$$

Making $f$ converge to the constant function 1 , we see that $x \in \mathcal{D}\left(\widehat{F}_{T}\right)$ and $\widehat{F}_{T} x=\widehat{F} x$. Thus we have $\widehat{F} \subset \widehat{F}_{T}$. The converse inclusion $\widehat{F}_{T} \subset \widehat{F}$ has already been known. 
Theorem 6.10. The operators $\widehat{\Delta}$ and $\widehat{J}$ (introduced by (5.1) and (5.17) respectively) coincide with the modular operator and the modular conjugation operator related to the GNS-mapping $\widehat{\eta}$. In particular,

$$
\begin{gathered}
\widehat{J} \widehat{A}^{\prime \prime} \widehat{J}=\widehat{A}^{\prime}, \\
\widehat{\Delta}^{i t} \widehat{A}^{\prime \prime} \widehat{\Delta}^{-i t}=\widehat{A}^{\prime \prime}
\end{gathered}
$$

hold for all $t \in \mathbb{R}$.

Proof. See formula (5.18).

Proposition 6.11. $A^{\prime \prime} \cap \widehat{A}^{\prime \prime}=\mathbb{C} 1$.

Proof. We know that $\widehat{J} \widehat{A}^{\prime \prime} \widehat{J}=\widehat{A}^{\prime}$ and $\widehat{J} A^{\prime \prime} \widehat{J}=A^{\prime \prime}$ (cf. (5.21)). Therefore $\widehat{J}\left(A^{\prime \prime} \cap \widehat{A^{\prime \prime}}\right) \widehat{J}=A^{\prime \prime} \cap \widehat{A}^{\prime}$ and our assertion reduces to Statement 3 of Proposition 4.7

\section{Proposition 6.12.}

(1) $\rho^{i t} \in M(A)$ for $t \in \mathbb{R}$ and the mapping $t \in \mathbb{R} \mapsto \rho^{i t} \in M(A)$ is strictly continuous.

(2) $\delta\left(\rho^{i t}\right)=\rho^{i t} \otimes \rho^{i t}$ for $t \in \mathbb{R}$.

(3) The operator $\gamma$ in Proposition 4.5 is a scalar multiple of the identity.

Proof. Ad 1 and 2. At first we shall prove that

$$
\delta^{M}\left(\rho^{i t}\right)=\rho^{i t} \otimes \rho^{i t} .
$$

Since $\widehat{\Delta}^{i t}=J \rho^{i t} J Q^{2 i t}$ for $t \in \mathbb{R}$ and $J \widehat{A} J=\widehat{A}$ by (5.23), it follows that $\rho^{i t}=J \widehat{\Delta}^{i t} Q^{-2 i t} J$ implements an action of $\mathbb{R}$ on $\widehat{A}^{\prime \prime}: \rho^{-i t} \widehat{A}^{\prime \prime} \rho^{i t} \subset \widehat{A}^{\prime \prime}$. Therefore the right hand side of the equation

$$
\left(\rho^{-i t} \otimes 1\right) \delta^{M}\left(\rho^{i t}\right)=\left(\rho^{-i t} \otimes 1\right) W\left(\rho^{i t} \otimes 1\right) W^{*},
$$

commutes with $\widehat{A}^{\prime} \otimes 1$. The left hand side clearly commutes with $A^{\prime} \otimes 1$. Since $A^{\prime \prime} \cap \widehat{A}^{\prime \prime}=\mathbb{C} 1$ by Proposition 6.11 the element in (6.16) is of the form $1 \otimes v_{t}$ for some unitary $v_{t}$. Hence $\delta^{M}\left(\rho^{i t}\right)=\rho^{i t} \otimes v_{t}$. It is easy to see that $\left\{v_{t}\right\}_{t \in \mathbb{R}}$ is a strongly continuous one parameter unitary group. Now we use a formula $\delta^{M} \circ R^{M}=\sigma \circ\left(R^{M} \otimes R^{M}\right) \circ \delta^{M}$. Since $R^{M}\left(\rho^{i t}\right)=\rho^{-i t}$ by Proposition 4.6] we find that $\delta^{M}\left(\rho^{i t}\right)=R^{M}\left(v_{-t}\right) \otimes \rho^{i t}$. Thus $\rho^{i t} \otimes v_{t}=R^{M}\left(v_{-t}\right) \otimes \rho^{i t}$. Therefore $v_{t}$ is a scalar multiple of $\rho^{i t}$. Hence there exists a positive scalar $\mu$ with $v_{t}=\mu^{i t} \rho^{i t}$. Inserting this $v_{t}$ to the last formula, we get $\mu^{-i t}=\mu^{i t}$ for all $t \in \mathbb{R}$. Hence we see that $\mu=1$ and $v_{t}=\rho^{i t}$.

Now formula (6.16) takes the form $1 \otimes \rho^{i t}=\left(\rho^{-i t} \otimes 1\right) W\left(\rho^{i t} \otimes 1\right) W^{*}$. All the factors on the right hand side belong to $M(\mathcal{K}(\mathcal{H}) \otimes A)$ and depend continuously on $t \in \mathbb{R}$ with respect to the strict topology. Therefore $\rho^{i t} \in M(A)$ and the mapping $t \mapsto \rho^{i t}$ is continuous with respect to the strict topology. At this moment one can erase the superscript $M$ in (6.15).

Ad 3. Due to (4.12) and (4.13) we have: $\sigma_{s}\left(\rho^{i t}\right)=\gamma^{i s t} \rho^{i t}$ and $\tau_{s}\left(\rho^{i t}\right)=\rho^{i t}$ for all $t, s \in \mathbb{R}$. Taking into account Assertion 2 of Lemma 4.3 we compute:

$$
\begin{aligned}
\delta\left(\gamma^{i s t}\right) \delta\left(\rho^{i t}\right) & =\delta\left(\gamma^{i s t} \rho^{i t}\right)=\delta\left(\sigma_{s}\left(\rho^{i t}\right)\right) \\
& =\left(\sigma_{s} \otimes \tau_{s}\right)\left(\delta\left(\rho^{i t}\right)\right)=\sigma_{s}\left(\rho^{i t}\right) \otimes \tau_{s}\left(\rho^{i t}\right) \\
& =\gamma^{i s t} \rho^{i t} \otimes \rho^{i t}=\left(\gamma^{i s t} \otimes 1\right) \delta\left(\rho^{i t}\right) .
\end{aligned}
$$

Thus we get $\delta\left(\gamma^{i s t}\right)=\gamma^{i s t} \otimes 1$. Using Proposition 4.7 we get $\gamma^{i s t} \in \mathbb{C} 1$. Hence $\gamma$ is a positive scalar multiple of the identity.

In short, Assertion 1 of Proposition 6.12 means that $\rho$ and $\rho^{-1}$ are affiliated to the $\mathrm{C}^{*}$-algebra $A$. Assertion 2 says that $\delta(\rho)=\rho \otimes \rho$. In other words

$$
W(\rho \otimes 1) W^{*}=\rho \otimes \rho .
$$

Proposition 6.13. We have

$$
\gamma=\lambda^{-1} 1
$$

where $\lambda$ is the constant appearing in Definition 1.5.

Proof. We shall use the first formula of (4.12). It shows that $\sigma_{s}\left(\rho^{i t}\right)=\gamma^{i s t} \rho^{i t}$ for any $s, t \in \mathbb{R}$. Therefore $\rho^{i t}$ is entire analytic for $\left\{\sigma_{s}\right\}_{s \in \mathbb{R}}$ and $\sigma_{i / 2}\left(\rho^{i t}\right)=\gamma^{-t / 2} \rho^{i t}$. By Theorem D.7 $b \rho^{i t} \in \mathcal{D}(\eta)$ and

$$
\gamma^{t / 2} \eta\left(b \rho^{i t}\right)=J\left(\rho^{i t}\right)^{*} J \eta(b)=J \rho^{-i t} J \eta(b)
$$


for any $b \in \mathcal{D}(\eta)$. Now we have:

$$
\widehat{\Delta}^{-i t} \eta(b)=Q^{-2 i t} J \rho^{-i t} J \eta(b)=\gamma^{t / 2} Q^{-2 i t} \eta\left(b \rho^{i t}\right)=(\lambda \gamma)^{t / 2} \eta\left(\tau_{-t}\left(b \rho^{i t}\right)\right),
$$

where in the last step we used (2.1).

Let $\varphi \in A^{*}$ be $L^{2}$-bounded. Taking into account (6.4) we get:

$$
\begin{aligned}
\left(\widehat{\Delta}^{i t} \widehat{\eta}\left(W_{\varphi}^{*}\right) \mid \eta(b)\right) & =\left(\widehat{\eta}\left(W_{\varphi}^{*}\right) \mid \widehat{\Delta}^{-i t} \eta(b)\right)=(\lambda \gamma)^{t / 2}\left(\widehat{\eta}\left(W_{\varphi}^{*}\right) \mid \eta\left(\tau_{-t}\left(b \rho^{i t}\right)\right)\right) \\
& =(\lambda \gamma)^{t / 2} \varphi\left(\tau_{-t}\left(b \rho^{i t}\right)\right)=(\lambda \gamma)^{t / 2}\left(\rho^{i t} \varphi^{\circ} \tau_{-t}\right)(b) .
\end{aligned}
$$

This formula holds for all $b \in \mathcal{D}(\eta)$. It shows that $\rho^{i t} \varphi^{\circ} \tau_{-t}$ is $L^{2}$-bounded and that

$$
\widehat{\Delta}^{i t} \widehat{\eta}\left(W_{\varphi}^{*}\right)=(\lambda \gamma)^{t / 2} \widehat{\eta}\left(W_{\rho^{i t} \varphi^{\circ} \tau_{-t}}^{*}\right) .
$$

On the other hand, according to the Tomita-Takesaki theory (Statement 1 of Theorem (D.6), $\widehat{\Delta}^{i t} \widehat{\eta}\left(W_{\varphi}^{*}\right)=\widehat{\eta}\left(\widehat{\sigma}_{t}\left(W_{\varphi}^{*}\right)\right)$, where $\widehat{\sigma}$ is the modular automorphism group related to the weight $\widehat{h}$ associated with $\widehat{\eta}$ (see (7.1) in the next section). Since $\widehat{\eta}$ is injective, we get

$$
\widehat{\sigma}_{t}\left(W_{\varphi}^{*}\right)=(\lambda \gamma)^{t / 2} W_{\rho^{i t} \varphi^{\circ} \tau_{-t}}^{*}
$$

Remembering that $\widehat{\sigma}_{t}$ and $\tau_{t}$ are implemented by $\widehat{\Delta}^{i t}$ and $Q^{2 i t}$ we obtain

$$
\begin{aligned}
(\mathrm{id} \otimes \varphi)\left(\left(\widehat{\Delta}^{i t} \otimes 1\right) W\left(\widehat{\Delta}^{-i t} \otimes 1\right)\right) & =(\lambda \gamma)^{t / 2}\left(\mathrm{id} \otimes\left(\rho^{i t} \varphi \circ \tau_{-t}\right)\right)(W) \\
& =(\lambda \gamma)^{t / 2}(\mathrm{id} \otimes \varphi)\left(\left(1 \otimes Q^{-2 i t}\right) W\left(1 \otimes Q^{2 i t} \rho^{i t}\right)\right) .
\end{aligned}
$$

Hence we have

$$
\left(\widehat{\Delta}^{i t} \otimes(\lambda \gamma)^{-t / 2} Q^{2 i t}\right) W=W\left(\widehat{\Delta}^{i t} \otimes Q^{2 i t} \rho^{i t}\right) .
$$

In this formula, all entries except $(\lambda \gamma)^{-t / 2}$ are unitary operators. Therefore $\left|(\lambda \gamma)^{-t / 2}\right|=1$ and $\gamma=\lambda^{-1} 1$.

Remark 6.14. Notice that the last formula of the above proof reduces now to

$$
\left(\widehat{\Delta}^{i t} \otimes Q^{2 i t}\right) W=W\left(\widehat{\Delta}^{i t} \otimes Q^{2 i t} \rho^{i t}\right) .
$$

At the end of this section we investigate the behavior of the right invariant Haar weight with respect to left translations. In the following proposition we use a vector presentation of the GNS map $\eta$ :

$$
\eta(a)=\sum_{k}^{\oplus} a \Omega_{k}
$$

for any $a \in \mathcal{D}(\eta)$. Then (cf. (3.11) $), W\left(\eta^{\prime \prime}(a) \otimes y\right)=\sum_{k}{ }^{\oplus} \delta(a)\left(\Omega_{k} \otimes y\right)$ for any $a \in \mathcal{D}\left(\eta^{\prime \prime}\right)$ and $y \in \mathcal{H}$.

Proposition 6.15. Let $a \in \mathcal{D}\left(\eta^{\prime \prime}\right)$ and $x \in \mathcal{D}\left(\rho^{1 / 2}\right)$. Then

$$
\sum_{k}^{\oplus} \delta(a)\left(x \otimes \Omega_{k}\right)=(\widehat{J} \otimes \widehat{J}) \widehat{W}^{*}(\widehat{J} \otimes \widehat{J})\left(\rho^{1 / 2} x \otimes \eta^{\prime \prime}(a)\right) .
$$

Proof. We may assume that $a \in \mathcal{D}\left(\eta^{\prime \prime}\right) \cap \mathcal{D}\left(\eta_{L}^{\prime \prime}\right)$ (by Proposition 5.14 the latter set is a core for $\left.\eta^{\prime \prime}\right)$. Using (5.31) we obtain:

$$
\begin{aligned}
W\left(\eta_{L}^{\prime \prime}(a) \otimes \widehat{J} \rho^{1 / 2} x\right) & =W\left(\lambda^{-i / 4} J \rho^{1 / 2} J \eta^{\prime \prime}(a) \otimes \widehat{J} \rho^{1 / 2} x\right) \\
& =\lambda^{-i / 4}(J \otimes \widehat{J}) W^{*}\left(\rho^{1 / 2} \otimes \rho^{1 / 2}\right)\left(J \eta^{\prime \prime}(a) \otimes x\right) \\
& =\lambda^{-i / 4}(J \otimes \widehat{J})\left(\rho^{1 / 2} \otimes 1\right) W^{*}\left(J \eta^{\prime \prime}(a) \otimes x\right) \\
& =\left(\lambda^{-i / 4} J \rho^{1 / 2} J \otimes 1\right) W\left(\eta^{\prime \prime}(a) \otimes \widehat{J} x\right) \\
& =\left(\lambda^{-i / 4} J \rho^{1 / 2} J \otimes 1\right) \sum_{k}^{\oplus} \delta(a)\left(\Omega_{k} \otimes \widehat{J} x\right)=\sum_{k}^{\oplus} \delta(a)\left(\widehat{J} \Omega_{k} \otimes \widehat{J} x\right) .
\end{aligned}
$$

In this computation we used in the second and fourth steps formula (5.22) and in the third step formula (6.17). At the end we used Proposition [5.15] It shows that the last series is convergent. 
Replacing in the above formula $a$ by $R(a)^{*}$ we obtain

$$
W\left(\widehat{J} \eta^{\prime \prime}(a) \otimes \widehat{J} \rho^{1 / 2} x\right)=\sum_{k}^{\oplus} \delta\left(R(a)^{*}\right)\left(\widehat{J} \Omega_{k} \otimes \widehat{J} x\right) .
$$

and

$$
\widehat{W}^{*}\left(\widehat{J} \rho^{1 / 2} x \otimes \widehat{J} \eta^{\prime \prime}(a)\right)=\sum_{k}^{\oplus} \sigma \circ \delta\left(R(a)^{*}\right)\left(\widehat{J} x \otimes \widehat{J} \Omega_{k}\right),
$$

where $\sigma$ denotes the flip acting on $A \otimes A$. Using Assertion 1 of Proposition 2.6 and formula (5.21) we obtain:

$$
\sigma \circ \delta\left(R(a)^{*}\right)=(R \otimes R) \delta\left(a^{*}\right)=(\widehat{J} \otimes \widehat{J}) \delta(a)(\widehat{J} \otimes \widehat{J}) .
$$

Therefore

$$
\widehat{W}^{*}\left(\widehat{J} \rho^{1 / 2} x \otimes \widehat{J} \eta^{\prime \prime}(a)\right)=\sum_{k}^{\oplus}(\widehat{J} \otimes \widehat{J}) \delta(a)\left(x \otimes \Omega_{k}\right)
$$

and (6.20) follows.

Proposition 6.16. Let $\varphi \in A_{+}^{*}$ and $b \in A_{+}$. Assume that $\varphi(\rho)<\infty$ and $h(b)<\infty$. Then

$$
h(b * \varphi)=\varphi(\rho) h(b) .
$$

It shows that $\rho$ is an analogue of the modular function on the group. It says, how the right invariant Haar measure transforms under left shifts.

Proof. Let $a=b^{1 / 2}$. Computing the norm of the both sides of (6.20) we obtain:

$$
\sum_{k}\left\|\delta(a)\left(x \otimes \Omega_{k}\right)\right\|^{2}=\left\|\rho^{1 / 2} x\right\|^{2} h\left(a^{*} a\right)=\omega_{x, x}(\rho) h(b) .
$$

On the other hand

$$
\sum_{k}\left\|\delta(a)\left(x \otimes \Omega_{k}\right)\right\|^{2}=\sum_{k}\left(x \otimes \Omega_{k}\left|\delta\left(a^{*} a\right)\right| x \otimes \Omega_{k}\right)=\sum_{k}\left(\Omega_{k}\left|b * \omega_{x, x}\right| \Omega_{k}\right)=h\left(b * \omega_{x, x}\right) .
$$

So we proved (6.21) for all $\varphi$ of the form $\omega_{x, x}\left(x \in \mathcal{D}\left(\rho^{1 / 2}\right)\right)$ i.e. for all $\varphi \in A_{*+}$ with $\varphi(\rho)<\infty$.

Let $\varphi \in A_{+}^{*}$. By the GNS construction one may assume that $\varphi(b)=\left(\Omega_{\varphi} \mid \pi(b) \Omega_{\varphi}\right)$ for any $b \in A$. In this formula $\pi$ is a representation of $A$ acting on a Hilbert space and $\Omega_{\varphi}$ is an element of this space. Applying the method developed in the proof of Theorem 3.9 to formula (6.20), one may show that

$$
\sum_{k}^{\oplus}(\pi \otimes \mathrm{id}) \delta(a)\left(\Omega_{\varphi} \otimes \Omega_{k}\right)=(\pi \otimes \mathrm{id})(\widehat{J} \otimes \widehat{J}) \widehat{W}^{*}(\widehat{J} \otimes \widehat{J})\left(\pi\left(\rho^{1 / 2}\right) \Omega_{\varphi} \otimes \eta^{\prime \prime}(a)\right) .
$$

Computing the norm of the both sides we obtain (6.21) in full generality. The details are left to the reader.

\section{Dual weighted Hopf $C^{*}$-Algebra and Duality}

In Section 3 applying Theorem 3.8 to the multiplicative unitary $\widehat{W}$, we introduced the proper $C^{*}$-bialgebra $(\widehat{A}, \widehat{\delta})$ with cancellation property together with the unitary antipode $\widehat{R}$ and the scaling group $\left\{\widehat{\tau}_{t}\right\}_{t \in \mathbb{R}}$ acting on $\widehat{A}$. In Section 6 we constructed the closed densely defined GNS map $\widehat{\eta}$ from $\widehat{A}$ into $\mathcal{H}$. Let $\widehat{h}$ be the locally finite lower semicontinuous weight on $\widehat{A}$ related to the GNS map $\widehat{\eta}$ by Theorem C.3 for any $b \in \widehat{A}$ we have

$$
\widehat{h}\left(b^{*} b\right)=\left\{\begin{array}{cc}
(\widehat{\eta}(b) \mid \widehat{\eta}(b)) & \text { if } b \in \mathcal{D}(\widehat{\eta}) \\
+\infty & \text { otherwise. }
\end{array}\right.
$$

Let $a \in \mathcal{D}\left(\widehat{\eta}^{\prime \prime}\right)$ and $\widehat{\eta}^{\prime \prime}(a)=0$. Then there exists a sequence $\left\{a_{n}\right\}_{n \in \mathbb{N}}$ of elements of $\mathcal{D}(\widehat{\eta})$ converging strongly to $a$ such that the sequence $\left\{\widehat{\eta}\left(a_{n}\right)\right\}_{n \in \mathbb{N}}$ converges in norm 0 . Taking into account (6.2) we obtain: $(y \mid a x)=(\eta(W(x, y)) \mid z)=0$ for all $(x, y) \in \Phi$. Remembering that $\Phi$ is dense in $\mathcal{H} \times \mathcal{H}$ we see that $a=0$. It shows that $\widehat{\eta}^{\prime \prime}$ is injective. Taking into account Lemma C.5 we conclude that $\widehat{h}$ is strictly faithful. 
We shall prove that $(\widehat{A}, \widehat{\delta})$ is a weighted Hopf $C^{*}$-algebra with $\widehat{h}$ playing the role of the right Haar weight. To this end we shall use Theorem 3.9 with $A, \delta, R, \tau, h$ and $W$ replaced by $\widehat{A}, \widehat{\delta}, \widehat{R}, \widehat{\tau}, \widehat{h}$ and $\widehat{W}=\Sigma W^{*} \Sigma$ respectively. With this replacement formulae (3.24) and (3.25) take the form:

$$
\begin{aligned}
\widehat{\eta}((\mathrm{id} \otimes \varphi) \widehat{\delta}(b)) & =((\mathrm{id} \otimes \varphi) \widehat{W}) \widehat{\eta}(b) \\
(\mathrm{id} \otimes \varphi \circ \widehat{\kappa}) \widehat{W} & =(\operatorname{id} \otimes \varphi)\left(\widehat{W}^{*}\right) .
\end{aligned}
$$

We have also to verify that for all $t \in \mathbb{R}$,

$$
\widehat{h} \circ \widehat{\tau}_{t}=\widehat{\lambda}^{t} \widehat{h}
$$

where $\widehat{\lambda}>0$ is a fixed number independent of $t$.

Lemma 7.1. Let $\varphi \in \widehat{A}^{*}$ and $b \in \mathcal{D}(\widehat{\eta})$. Then $(\mathrm{id} \otimes \varphi) \widehat{\delta}(b) \in \mathcal{D}(\widehat{\eta})$ and formula (17.2) holds.

Proof. According to Corollary 6.8 the set $\widehat{A}_{0}$ (cf. 6.5) is a core for $\widehat{\eta}$. Therefore we may assume that $b=W_{\psi}^{*}$ where $\psi$ is a $L^{2}$-bounded functional on $A^{*}$. Applying id $\otimes \mathrm{id} \otimes \psi^{*}$ to the both sides of (3.21) we get $\widehat{\delta}(b)=\left(\mathrm{id} \otimes \mathrm{id} \otimes \psi^{*}\right)\left(W_{13}^{*} W_{23}^{*}\right)$. Setting $d=(\varphi \otimes \mathrm{id})\left(W^{*}\right) \in M(A)$ we obtain

$$
\begin{aligned}
(\mathrm{id} \otimes \varphi) \widehat{\delta}(b) & =\left(\mathrm{id} \otimes \varphi \otimes \psi^{*}\right)\left(W_{13}^{*} W_{23}^{*}\right) \\
& =\left(\mathrm{id} \otimes \psi^{*}\right)\left(W^{*}(1 \otimes d)\right) \\
& =\left(\mathrm{id} \otimes d \psi^{*}\right)\left(W^{*}\right)=W_{\psi d^{*}}^{*} .
\end{aligned}
$$

We have to show that

$$
W_{\psi d^{*}}^{*} \in \mathcal{D}(\widehat{\eta}) \quad \text { and } \quad \widehat{\eta}\left(W_{\psi d^{*}}^{*}\right)=d \widehat{\eta}(b) .
$$

We shall use formula (6.4). For any $a \in \mathcal{D}(\eta)$, we have

$$
\left(\psi d^{*}\right)(a)=\psi\left(d^{*} a\right)=\left(\widehat{\eta}\left(W_{\psi}^{*}\right) \mid \eta\left(d^{*} a\right)\right)=\left(d \widehat{\eta}\left(W_{\psi}^{*}\right) \mid \eta(a)\right) .
$$

Now, (7.5) follows immediately from Assertion 4 of Theorem 6.4

Lemma 7.2. Formula (7.3) holds for any $\varphi \in \widehat{A}^{*}$ such that $\varphi \circ \widehat{\kappa} \in \widehat{A}^{*}$.

Proof. Let $\psi \in \mathcal{L}(\mathcal{H})_{*}$. Formula (3.18) applied to the manageable multiplicative unitary $\widehat{W}$ shows that $(\psi \otimes \mathrm{id})(\widehat{W}) \in \mathcal{D}(\widehat{\kappa})$ and

$$
\widehat{\kappa}((\psi \otimes \mathrm{id})(\widehat{W}))=(\psi \otimes \mathrm{id})\left(\widehat{W}^{*}\right) .
$$

Therefore we have

$$
\begin{aligned}
\psi((\operatorname{id} \otimes \varphi \circ \widehat{\kappa})(\widehat{W})) & =(\varphi \circ \widehat{\kappa})((\psi \otimes \mathrm{id})(\widehat{W})) \\
& =\varphi\left((\psi \otimes \mathrm{id})\left(\widehat{W}^{*}\right)\right)=\psi\left((\mathrm{id} \otimes \varphi)\left(\widehat{W}^{*}\right)\right)
\end{aligned}
$$

and (7.3) follows.

Formula (7.4) with $\widehat{\lambda}=\lambda^{-1}$ follows immediately from the following

Proposition 7.3. Let $b \in \mathcal{D}(\widehat{\eta})$. Then $\widehat{\tau}_{t}(b) \in \mathcal{D}(\widehat{\eta})$ and

$$
\widehat{\eta}\left(\widehat{\tau}_{t}(b)\right)=\lambda^{-t / 2} Q^{2 i t} \widehat{\eta}(b)
$$

for all $t \in \mathbb{R}$.

Proof. Assume that $b \in \mathcal{D}(\widehat{\eta})$. Then

$$
(y \mid b x)=(\eta(W(x, y)) \mid \widehat{\eta}(b))
$$

for all $(x, y) \in \Phi$. Let $t \in \mathbb{R}$. According to the second assertion of Lemma $2.8 \tau_{-t}(W(x, y))=$ $W\left(Q^{-2 i t} x, Q^{-2 i t} y\right)$. Formula (2.2) shows now that $\left(Q^{-2 i t} x, Q^{-2 i t} y\right) \in \Phi$ and

$$
\eta\left(W\left(Q^{-2 i t} x, Q^{-2 i t} y\right)\right)=\lambda^{-t / 2} Q^{-2 i t} \eta((W(x, y)) .
$$

Replacing $(x, y)$ in (7.6) by $\left(Q^{-2 i t} x, Q^{-2 i t} y\right)$, we obtain

$$
\left(y \mid \widehat{\tau}_{t}(b) x\right)=\left(\eta(W(x, y)) \mid \lambda^{-t / 2} Q^{2 i t} \widehat{\eta}(b)\right)
$$

for all $(x, y) \in \Phi$. It shows that $\widehat{\tau}_{t}(b) \in \mathcal{D}(\widehat{\eta})$ and $\widehat{\eta}\left(\widehat{\tau}_{t}(b)\right)=\lambda^{-t / 2} Q^{2 i t} \widehat{\eta}(b)$.

Using now Theorem 3.9 we get 
Theorem 7.4. The $C^{*}$-bialgebra $(\widehat{A}, \widehat{\delta})$ is a weighted Hopf $C^{*}$-algebra with antipode $\widehat{\kappa}$, Haar weight $\widehat{h}$ and scaling constant $\widehat{\lambda}$ equal to the inverse of the corresponding constant $\lambda$ for $(A, \delta)$.

By definition $(\widehat{A}, \widehat{\delta})$ is the dual of the weighted Hopf $C^{*}$-algebra $(A, \delta)$. It is easy to see that the passage $W \longmapsto \widehat{W}$ is involutive:

$$
\widehat{\widehat{W}}=\Sigma \widehat{W}^{*} \Sigma=\Sigma\left(\Sigma W^{*} \Sigma\right)^{*} \Sigma=W .
$$

Therefore we have the following

Theorem 7.5 (Duality). The dual of the dual of a weighted Hopf $C^{*}$-algebra is isomorphic to the original weighted Hopf $C^{*}$-algebra.

Developing the theory of weighted Hopf $C^{*}$-algebras we constructed a number of objects related to a given weighted Hopf $C^{*}$-algebra $(A, \delta)$. We list some of them in order of appearance:

$$
\kappa, h, \tau, R, \lambda, \mathcal{H}, \eta, Q, \Delta, J, W, \widehat{A}, \widehat{\delta}, \widehat{R}, \widehat{\tau}, \widehat{\Delta}, \widehat{J} \text { and } \widehat{\eta} \text {. }
$$

Clearly the corresponding objects related to the dual weighted Hopf $C^{*}$-algebra $(\widehat{A}, \widehat{\delta})$ equals:

$$
\widehat{\kappa}, \widehat{h}, \widehat{\tau}, \widehat{R}, \widehat{\lambda}=\lambda^{-1}, \mathcal{H}, \widehat{\eta}, Q, \widehat{\Delta}, \widehat{J}, \widehat{W}=\Sigma W^{*} \Sigma, A, \delta, R, \tau, \Delta, J \text { and } \eta .
$$

The objects dual to $\rho$ and $\gamma$ will be denoted by $\widehat{\rho}$ and $\widehat{\gamma}$, respectively. Many of the formulae appearing in the paper form dual pairs. The examples are: ([3.8), (3.21) $),($ (5.25), (2.4) $),($ (5.26), (5.21) $)$, etc. The formulae (5.22), (3.9), (7.7), etc. are selfdual.

Proposition 7.6. Let $\widehat{\Delta}_{\text {rel }}$ be the relative modular operator determined by the weight $\widehat{h}$ and the weight $\widehat{h} \circ \widehat{R}\left(\widehat{\Delta}_{\mathrm{rel}}\right.$ is the object dual to $\left.\Delta_{\mathrm{rel}}\right)$. Then

$$
\widehat{\Delta}_{\text {rel }} \otimes \Delta=W\left(\widehat{\Delta} \otimes \Delta_{\text {rel }}\right) W^{*} .
$$

Proof. We start with formula (6.17). Applying to the both sides $\mathrm{Ad}_{J \otimes \widehat{J}}$ and using (5.22) we obtain

$$
W^{*}(J \rho J \otimes 1) W=J \rho J \otimes \widehat{J} \rho \widehat{J} .
$$

Combining Proposition 4.6] with [5.21) we see that $\widehat{J} \rho \widehat{J}=\rho^{-1}$. Therefore

$$
W^{*}(J \rho J \otimes 1) W=J \rho J \otimes \rho^{-1} .
$$

We shall also use the dual formula

$$
\widehat{W}^{*}(\widehat{J} \widehat{\rho} \widehat{J} \otimes 1) \widehat{W}=\widehat{J} \widehat{\rho} \widehat{J} \otimes \widehat{\rho}^{-1}
$$

By easy computation it reduces to

$$
W^{*}\left(\widehat{\rho}^{-1} \otimes \widehat{J} \widehat{\rho} \widehat{J}\right) W=1 \otimes \widehat{J} \widehat{\rho} \widehat{J} .
$$

Let $t \in \mathbb{R}$. Rising both sides of (7.8) and (7.9) to the power -it and multiplying side by side the two equations we obtain

$$
W^{*}\left(J \rho^{i t} J \widehat{\rho}^{i t} \otimes \widehat{J} \widehat{\rho}^{i t} \widehat{J}\right) W=J \rho^{i t} J \otimes \rho^{i t} \widehat{J} \widehat{\rho}^{i t} \widehat{J} .
$$

Remembering that $W$ commutes with $Q \otimes Q$ we get

$$
W^{*}\left(J \rho^{i t} J \widehat{\rho}^{i t} Q^{2 i t} \otimes \widehat{J} \widehat{\rho}^{i t} \widehat{J} Q^{2 i t}\right) W=J \rho^{i t} J Q^{2 i t} \otimes \rho^{i t} \widehat{J} \widehat{\rho}^{i t} \widehat{J} Q^{2 i t} .
$$

Formula (5.1) shows that $J \rho^{i t} J Q^{2 i t}=\widehat{\Delta}^{i t}$. By duality $\widehat{J} \widehat{\rho}^{i t} \widehat{J} Q^{2 i t}=\Delta^{i t}$. Consequently, by (4.14) and (6.18) we have $\rho^{i t} \widehat{J} \widehat{\rho}^{i t} \widehat{J} Q^{2 i t}=\rho^{i t} \Delta^{i t}=\gamma^{-i t^{2} / 2} \Delta_{\text {rel }}^{i t}=\lambda^{i t^{2} / 2} \Delta_{\text {rel }}^{i t}$. By duality $\hat{\rho}^{i t} J \rho^{i t} J Q^{2 i t}=\lambda^{-i t^{2} / 2} \widehat{\Delta}_{\text {rel }}^{i t}$. Replacing $t$ by $-t$ and taking hermitian conjugation we obtain $J \rho^{i t} J Q^{2 i t} \widehat{\rho}^{i t}=\lambda^{i t^{2} / 2} \widehat{\Delta}_{\text {rel }}^{i t}$. We know (cf. (4.13) ) that $\rho$ is $\tau_{t}$-invariant. Therefore it commutes with $Q$. So does $\widehat{\rho}$ (duality!). Therefore $J \rho^{i t} J \hat{\rho}^{i t} Q^{2 i t}=\lambda^{i t^{2} / 2} \widehat{\Delta}_{\text {rel }}^{i t}$. Inserting these data into (7.10) we obtain:

and (7.7) follows.

$$
W^{*}\left(\widehat{\Delta}_{\text {rel }}^{i t} \otimes \Delta^{i t}\right) W=\widehat{\Delta}^{i t} \otimes \Delta_{\text {rel }}^{i t}
$$

We end this Section with an explicit formula for the relative Tomita-Takesaki operator $S_{\text {rel }}$. By definition $S_{\text {rel }}$ is a closed conjugate linear operator acting on $\mathcal{H}$ such that

$$
S_{\text {rel }} b^{*} \eta(a)=a^{*} \eta_{L}(b)
$$


for any $a \in \mathcal{D}\left(\eta^{\prime \prime}\right)$ and $b \in \mathcal{D}\left(\eta_{L}^{\prime \prime}\right)$. The self-adjoint part of the polar decomposition of $S_{\text {rel }}$, denoted by $\Delta_{\text {rel }}^{1 / 2}$, has been already investigated in Section 4 Let $J_{\text {rel }}$ be the antiunitary part of this decomposition:

$$
S_{\mathrm{rel}}=J_{\mathrm{rel}} \Delta_{\mathrm{rel}}^{1 / 2}
$$

We shall prove that $J_{\text {rel }}=\lambda^{-i / 8} J$. Let $a \in \mathcal{D}\left(\eta^{\prime \prime}\right)$ and $b \in \mathcal{D}\left(\eta_{L}^{\prime \prime}\right) \cap \mathcal{D}\left(\eta^{\prime \prime}\right)$. Then we have

$$
\begin{aligned}
J J_{\mathrm{rel}}^{*} a^{*} \eta_{L}^{\prime \prime}(b) & =J J_{\mathrm{rel}}^{*} S_{\mathrm{rel}} b^{*} \eta^{\prime \prime}(a)=J \Delta_{\text {rel }}^{1 / 2} b^{*} \eta^{\prime \prime}(a) \\
& =\gamma^{i / 8} J \rho^{1 / 2} \Delta^{1 / 2} b^{*} \eta^{\prime \prime}(a)=\gamma^{i / 8} J \rho^{1 / 2} J a^{*} \eta^{\prime \prime}(b) \\
& =\gamma^{i / 8} \lambda^{i / 4} \eta_{L}^{\prime \prime}\left(a^{*} b\right)=\lambda^{i / 8} a^{*} \eta_{L}^{\prime \prime}(b),
\end{aligned}
$$

where we used (4.15) in the third step, (5.31) in the fifth step and (6.18) in the last step.

Now we notice that due to the last statement of Proposition 5.14 $a^{*} \eta_{L}^{\prime \prime}(b)$ runs over a dense subset of $\mathcal{H}$. Therefore $J J_{\text {rel }}^{*}=\lambda^{i / 8} 1, J_{\text {rel }}=\lambda^{-i / 8} J$. In this way we showed that

$$
S_{\mathrm{rel}}=\lambda^{-i / 8} J \Delta_{\mathrm{rel}}^{1 / 2}
$$

\section{Quantum Codouble}

In 22] P. Podleś and the third author of the present paper have introduced the so called 'double group construction'. It was the construction dual to the quantum double of Drinfeld [6]. In this section we describe this construction within the framework of weighted Hopf $C^{*}$-algebras. The weighted Hopf $\mathrm{C}^{*}$-algebra $\left(B, \delta^{B}\right)$ arising with this construction is built of a weighted Hopf $C^{*}$ algebra $(A, \delta)$ and its dual $(\widehat{A}, \widehat{\delta})$. We shall call $\left(B, \delta^{B}\right)$ the quantum codouble of $(A, \delta)$. The theory presented in [22] deals with the case of compact $(\widehat{A}, \widehat{\delta})$ (and discrete $(A, \delta)$ ).

A weighted Hopf $C^{*}$-algebra is said to be unimodular, if the right Haar weight is left invariant. Then $h \circ R=h$.

Let $(A, \delta)$ be a weighted Hopf $C^{*}$-algebra, $(\widehat{A}, \widehat{\delta})$ be its dual and $W \in M(\widehat{A} \otimes A)$ be the KacTakesaki operator related to $(A, \delta)$. We shall consider the twisted flip $\sigma_{W}$ acting from $\widehat{A} \otimes A$ to $A \otimes \widehat{A}$ defined by

$$
\sigma_{W}=\sigma \circ \operatorname{Ad}_{W^{*}}=\operatorname{Ad}_{\hat{W}^{\circ} \sigma}
$$

where $\sigma$ is the flip map: $\sigma(b \otimes a)=a \otimes b$ for any $a \in A$ and $b \in \widehat{A}$. Then $\sigma_{W} \in \operatorname{Mor}(\widehat{A} \otimes A, A \otimes \widehat{A})$.

The quantum codouble of $(A, \delta)$ is by definition the pair $\left(B, \delta^{B}\right)$, where

$$
\begin{aligned}
B & =\widehat{A} \otimes A \\
\delta^{B} & =\left(\mathrm{id} \otimes \sigma_{W} \otimes \mathrm{id}\right) \circ(\widehat{\delta} \otimes \delta) .
\end{aligned}
$$

In the second formula $\widehat{\delta} \otimes \delta \in \operatorname{Mor}(\widehat{A} \otimes A, \widehat{A} \otimes \widehat{A} \otimes A \otimes A)=\operatorname{Mor}(B, \widehat{A} \otimes \widehat{A} \otimes A \otimes A)$ and id $\otimes \sigma_{W} \otimes$ id $\in \operatorname{Mor}(\widehat{A} \otimes \widehat{A} \otimes A \otimes A, \widehat{A} \otimes A \otimes \widehat{A} \otimes A)=\operatorname{Mor}(\widehat{A} \otimes \widehat{A} \otimes A \otimes A, B \otimes B)$. Therefore $\delta^{B} \in \operatorname{Mor}(B, B \otimes B)$. We start with the following

Proposition 8.1. The pair $\left(B, \delta^{B}\right)$ is a proper $C^{*}$-bialgebra with the cancellation property.

Proof. Clearly $B$ is a separable $\mathrm{C}^{*}$-algebra. Using formulae (3.8) and (3.21) one can easily verify that

$$
\begin{aligned}
& (\delta \otimes \mathrm{id}) \sigma_{W}=\left(\mathrm{id} \otimes \sigma_{W}\right)\left(\sigma_{W} \otimes \mathrm{id}\right)(\mathrm{id} \otimes \delta), \\
& (\mathrm{id} \otimes \widehat{\delta}) \sigma_{W}=\left(\sigma_{W} \otimes \mathrm{id}\right)\left(\mathrm{id} \otimes \sigma_{W}\right)(\widehat{\delta} \otimes \mathrm{id}) .
\end{aligned}
$$

Now the coassociativity of $\delta^{B}$ follows from a simple computation. For details see [22, the proof of Theorem 4.1].

For any subset $E \subset M(B \otimes B)$ we denote by $\operatorname{CLS}\{E\}$ the norm-closed linear span of $E$. To prove the properness and the cancellation property we have to show that CLS $\left\{\delta^{B}(B)(1 \otimes B)\right\}=$ $\operatorname{CLS}\left\{(B \otimes 1) \delta^{B}(B)\right\}=B \otimes B$. 
We know that the set $\delta(A)(1 \otimes A)$ is a linearly dense subset of $A \otimes A$. Therefore

$$
\begin{aligned}
\operatorname{CLS}\left\{\delta^{B}(B)(1 \otimes B)\right\} & =\Sigma_{23} \operatorname{CLS}\left\{W_{23}^{*}(\widehat{\delta}(\widehat{A}) \otimes \delta(A)) W_{23}(1 \otimes \widehat{A} \otimes 1 \otimes A)\right\} \Sigma_{23} \\
& =\Sigma_{23} \operatorname{CLS}\left\{W_{23}^{*}(\widehat{\delta}(\widehat{A}) \otimes \delta(A)(1 \otimes A)) W_{23}(1 \otimes \widehat{A} \otimes 1 \otimes 1)\right\} \Sigma_{23} \\
& =\Sigma_{23} \operatorname{CLS}\left\{W_{23}^{*}(\widehat{\delta}(\widehat{A}) \otimes A \otimes A) W_{23}(1 \otimes \widehat{A} \otimes 1 \otimes 1)\right\} \Sigma_{23} \\
& =\Sigma_{23} \operatorname{CLS}\left\{W_{23}^{*} \widehat{W}_{12}(\widehat{A} \otimes 1 \otimes A \otimes A) \widehat{W}_{12}^{*} W_{23}(1 \otimes \widehat{A} \otimes 1 \otimes 1)\right\} \Sigma_{23},
\end{aligned}
$$

where in the last step we used the formula $\widehat{\delta}(b)=\widehat{W}(b \otimes 1) \widehat{W}^{*}$. The pentagonal equation implies that $\widehat{W}_{12}^{*} W_{23}=W_{13} \widehat{W}_{12}^{*} W_{13}^{*}$. Therefore using in the second step the formula $(\widehat{A} \otimes A) W=(\widehat{A} \otimes A)$ we obtain

$$
\begin{aligned}
\operatorname{CLS}\left\{\delta^{B}(B)(1 \otimes B)\right\} & =\Sigma_{23} \operatorname{CLS}\left\{W_{23}^{*} \widehat{W}_{12}(\widehat{A} \otimes 1 \otimes A \otimes A) W_{13} \widehat{W}_{12}^{*} W_{13}^{*}(1 \otimes \widehat{A} \otimes 1 \otimes 1)\right\} \Sigma_{23} \\
& =\Sigma_{23} \operatorname{CLS}\left\{W_{23}^{*} \widehat{W}_{12}(\widehat{A} \otimes 1 \otimes A \otimes A) \widehat{W}_{12}^{*} W_{13}^{*}(1 \otimes \widehat{A} \otimes 1 \otimes 1)\right\} \Sigma_{23} \\
& =\Sigma_{23} W_{23}^{*} \operatorname{CLS}\{(\widehat{\delta}(\widehat{A}) \otimes A \otimes A)(1 \otimes \widehat{A} \otimes 1 \otimes 1)\} W_{13}^{*} \Sigma_{23} .
\end{aligned}
$$

We know that the set $\widehat{\delta}(\widehat{A})(1 \otimes \widehat{A})$ is a linearly dense subset of $\widehat{A} \otimes \widehat{A}$. Therefore

$$
\begin{aligned}
\operatorname{CLS}\left\{\delta^{B}(B)(1 \otimes B)\right\} & =\Sigma_{23} W_{23}^{*}(\widehat{A} \otimes \widehat{A} \otimes A \otimes A) W_{13}^{*} \Sigma_{23} \\
& =\Sigma_{23}(\widehat{A} \otimes \widehat{A} \otimes A \otimes A) \Sigma_{23} \\
& =\widehat{A} \otimes A \otimes \widehat{A} \otimes A=B \otimes B .
\end{aligned}
$$

In the similar way one can show that $\operatorname{CLS}\left\{(B \otimes 1) \delta^{B}(B)\right\}=B \otimes B$.

Let $\eta, \widehat{\eta}$ and $\eta_{L}$ be the GNS maps considered in previous sections. We shall also use the GNS map $\widehat{\eta}_{L}$ defined on $\widehat{A}$, introduced by the formula

$$
\widehat{\eta}_{L}(b)=J \widehat{\eta}\left(\widehat{R}\left(b^{*}\right)\right)
$$

dual to (5.27). By definition $\mathcal{D}\left(\widehat{\eta}_{L}\right)=\left\{b \in \widehat{A}: \widehat{R}\left(b^{*}\right) \in \mathcal{D}(\widehat{\eta})\right\}$. As we know, $\eta$ and $\eta_{L}$ are GNS maps related to the right and left Haar weights $h$ and $h \circ R$ on $(A, \delta)$. In same way, $\widehat{\eta}$ and $\widehat{\eta}_{L}$ are GNS maps related to the right and left Haar weights $\widehat{h}$ and $\widehat{h}$ 。 $\widehat{R}$ on $(\widehat{A}, \widehat{\delta})$. We shall use the tensor product of GNS mappings (see Appendix E). Let

$$
\begin{aligned}
& \eta^{B}=\widehat{\eta}_{L} \otimes \eta, \\
& \eta_{d}^{B}=\widehat{\eta} \otimes \eta_{L} .
\end{aligned}
$$

Then $\eta^{B}$ and $\eta_{d}^{B}$ are closed densely defined GNS maps on $B=\widehat{A} \otimes A$ with values in $\mathcal{H} \otimes \mathcal{H}$. Let $U \in \mathcal{L}(\mathcal{H} \otimes \mathcal{H})$ be the unitary operator introduced by

$$
U=W(\widehat{J} \otimes J) W(\widehat{J} \otimes J) .
$$

We recall that $W \in M(\widehat{A} \otimes A)=M(B)$.

Proposition 8.2. We have: $\mathcal{D}\left(\eta_{d}^{B}\right)=\operatorname{Ad}_{W^{*}}\left(\mathcal{D}\left(\eta^{B}\right)\right)$ and

$$
U^{*} \eta^{B}(c)=\lambda^{i / 4} \eta_{d}^{B}\left(\operatorname{Ad}_{W^{*}}(c)\right)
$$

for any $c \in \mathcal{D}\left(\eta^{B}\right)$.

Proof. Recall that $S_{\text {rel }} \eta(a)=\eta_{L}\left(a^{*}\right)$ for $a \in \mathcal{D}(\eta) \cap \mathcal{D}\left(\eta_{L}\right)^{*}$ and (cf. (7.11) ) $S_{\text {rel }}=\lambda^{-i / 8} J \Delta_{\text {rel }}^{1 / 2}$. By duality $\widehat{S}_{\mathrm{rel}} \widehat{\eta}(a)=\widehat{\eta}_{L}\left(a^{*}\right)$ for $a \in \mathcal{D}(\widehat{\eta}) \cap \mathcal{D}\left(\widehat{\eta}_{L}\right)^{*}$ and $\widehat{S}_{\mathrm{rel}}=\lambda^{i / 8} \widehat{J}_{\Delta_{\text {rel }}^{1 / 2}}$. To make the notation shorter we set $J_{B}=\widehat{J} \otimes J$. For any $a \in \mathcal{D}(\eta) \cap \mathcal{D}\left(\eta_{L}\right)^{*}$ and $b \in \mathcal{D}(\widehat{\eta}) \cap \mathcal{D}(\widehat{\eta})^{*}$ we have

$$
\begin{aligned}
U \eta_{d}^{B}\left(b^{*} \otimes a^{*}\right) & =W J_{B} W J_{B}\left(\widehat{\eta}\left(b^{*}\right) \otimes \eta_{L}\left(a^{*}\right)\right)=W J_{B} W J_{B}\left(\widehat{S} \otimes S_{\mathrm{rel}}\right)(\widehat{\eta}(b) \otimes \eta(a)) \\
& =\lambda^{-i / 8} W J_{B} W\left(\widehat{\Delta} \otimes \Delta_{\mathrm{rel}}\right)^{1 / 2}(\widehat{\eta}(b) \otimes \eta(a)) \\
& =\lambda^{-i / 8} W J_{B}\left(\widehat{\Delta}_{\mathrm{rel}} \otimes \Delta\right)^{1 / 2} W(\widehat{\eta}(b) \otimes \eta(a)) \\
& =\lambda^{-i / 8} W J_{B}\left(\widehat{\Delta}_{\mathrm{rel}} \otimes \Delta\right)^{1 / 2}(\widehat{\eta} \otimes \eta)(W(b \otimes a)) \\
& =\lambda^{-i / 4} W\left(\widehat{S}_{\mathrm{rel}} \otimes S\right)(\widehat{\eta} \otimes \eta)(W(b \otimes a))
\end{aligned}
$$


where in the fourth step we used relation (7.7). Since $W(b \otimes a) \in \mathcal{D}(\widehat{\eta} \otimes \eta)$ and $(\widehat{\eta} \otimes \eta)(W(b \otimes a)) \in$ $\mathcal{D}\left(\widehat{S}_{\text {rel }} \otimes S\right)$, it follows (cf. Theorem D.5) that $(b \otimes a)^{*} W^{*} \in \mathcal{D}\left(\widehat{\eta}_{L} \otimes \eta\right)$. Hence we see that

$$
\begin{aligned}
U \eta_{d}^{B}\left(b^{*} \otimes a^{*}\right) & =\lambda^{-i / 4} W\left(\widehat{S}_{\text {rel }} \otimes S\right)(\widehat{\eta} \otimes \eta)(W(b \otimes a)) \\
& =\lambda^{-i / 4} W\left(\widehat{\eta}_{L} \otimes \eta\right)\left(\left(b^{*} \otimes a^{*}\right) W^{*}\right) \\
& =\lambda^{-i / 4}\left(\widehat{\eta}_{L} \otimes \eta\right)\left(W\left(b^{*} \otimes a^{*}\right) W^{*}\right)=\lambda^{-i / 4} \eta^{B}\left(\operatorname{Ad}_{W}\left(b^{*} \otimes a^{*}\right)\right) .
\end{aligned}
$$

Since the algebraic tensor product of $\mathcal{D}(\widehat{\eta}) \cap \mathcal{D}(\widehat{\eta})^{*}$ and $\mathcal{D}\left(\eta_{L}\right) \cap \mathcal{D}(\eta)^{*}$ is a core for the GNS mapping $\eta_{d}^{B}$, it follows from the above argument that $\operatorname{Ad}_{W}(c) \in \mathcal{D}\left(\eta^{B}\right)$ and

$$
U \eta_{d}^{B}(c)=\lambda^{-i / 4} \eta^{B}\left(\operatorname{Ad}_{W}(c)\right)
$$

for any $c \in \mathcal{D}\left(\eta_{d}^{B}\right)$. Hence we see that $\operatorname{Ad}_{W}\left(\mathcal{D}\left(\eta_{d}^{B}\right)\right) \subset \mathcal{D}\left(\eta^{B}\right)$. Passing to the dual statement we obtain $\operatorname{Ad}_{W^{*}}\left(\mathcal{D}\left(\eta^{B}\right)\right) \subset \mathcal{D}\left(\eta_{d}^{B}\right)$. Combining the two inclusions we obtain the equality $\operatorname{Ad}_{W^{*}}\left(\mathcal{D}\left(\eta^{B}\right)\right)=\mathcal{D}\left(\eta_{d}^{B}\right)$. To end the proof we notice that (8.6) is equivalent to (8.5).

Now we shall introduce the unitary antipode, the scaling group and the Haar weight related to $\left(B, \delta^{B}\right)$ :

$$
\left.\begin{array}{rl}
R^{B} & =(\widehat{R} \otimes R) \circ \mathrm{Ad}_{W^{*}}, \\
\tau_{t}^{B} & =\widehat{\tau}_{t} \otimes \tau_{t}, \\
h^{B} & =\widehat{h}^{\circ} \widehat{R} \otimes h,
\end{array}\right\}
$$

where $h^{B}=\widehat{h} \circ \widehat{R} \otimes h$ is the weight on $B$ associated with the GNS mapping $\eta^{B}=\widehat{\eta}_{L} \otimes \eta$. Using the properties

$$
(\widehat{R} \otimes R)(W)=W, \quad(\widehat{R} \otimes R) \circ \operatorname{Ad}_{W^{*}}=\operatorname{Ad}_{W^{\circ}}(\widehat{R} \otimes R), \quad\left(\widehat{\tau}_{t} \otimes \tau_{t}\right)(W)=W, \quad \widehat{\lambda} \lambda=1
$$

one can easily verify the following:

Proposition 8.3. $R^{B}$ is an involutive antiautomorphism of $B,\left\{\tau_{t}^{B}\right\}_{t \in \mathbb{R}}$ is a strongly continuous one parameter group of automorphisms of $B$ commuting with $R^{B}$ and $h^{B}$ is a locally finite, strictly faithful, lower semicontinuous weight on $B$ such that $h^{B}{ }_{o} \tau_{t}^{B}=h^{B}$ for all $t \in \mathbb{R}$. Moreover

$$
h^{B}{ }_{o} R^{B}=h^{B} \text {. }
$$

Proof. Only the last formula needs a justification. Let $h_{d}^{B}$ be the weight on $B$ related to the GNS map $\eta_{d}^{B}$. By definition

$$
h_{d}^{B}=\widehat{h} \otimes h \circ R=(\widehat{h} \circ \widehat{R} \otimes h) \circ(\widehat{R} \otimes R)=h^{B} \circ(\widehat{R} \otimes R) .
$$

Therefore

$$
h^{B} \circ R^{B}=h_{d}^{B} \circ \operatorname{Ad}_{W^{*}}=h^{B},
$$

where the last equality follows immediately from 8.5 .

Our main aim in this section is to show that $\left(B, \delta^{B}\right)$ is a weighted Hopf $\mathrm{C}^{*}$-algebra. To this end we shall use Theorem 3.9 with $A, \delta, R, \tau$ and $h$ replaced by $B, \delta^{B}, R^{B}, \tau^{B}$ and $h^{B}$. Then the role of $\mathcal{H}$ and $\eta$ is played by $\mathcal{H}^{B}=\mathcal{H} \otimes \mathcal{H}$ and $\eta^{B}$ and the unitary $W$ (denoted in the present case by $\left.W^{B}\right)$ acts on $\mathcal{H}^{B} \otimes \mathcal{H}^{B}=\mathcal{H} \otimes \mathcal{H} \otimes \mathcal{H} \otimes \mathcal{H}$. We shall prove that

$$
W^{B}=U_{12} \widehat{W}_{13} U_{12}^{*} W_{24} \text {. }
$$

satisfies all the assumptions of Theorem 3.9 Remembering that $W \in M(\widehat{A} \otimes A) \subset M(\mathcal{K}(\mathcal{H}) \otimes A)$ and $\widehat{W} \in M(A \otimes \widehat{A}) \subset M(\mathcal{K}(\mathcal{H}) \otimes \widehat{A})$ we see that $W^{B} \in M(\mathcal{K}(\mathcal{H}) \otimes \mathcal{K}(\mathcal{H}) \otimes \widehat{A} \otimes A)$ and

$$
W^{B} \in M\left(\mathcal{K}\left(\mathcal{H}^{B}\right) \otimes B\right) .
$$

In the following propositions we shall use a special notation. For any $\varphi \in A^{*}$ and any $a \in A$ we set: $\varphi_{*}(a)=\varphi * a$. Then $\varphi_{*}$ is a linear mapping acting on $A$. Similarly for any $\psi \in \widehat{A}^{*}$ and any $b \in \widehat{A}$ we set: $\psi_{*}(b)=\psi * b$. Then $\psi_{*}$ is a linear mapping acting on $\widehat{A}$. Clearly

$$
\begin{aligned}
& \varphi_{*}=(\mathrm{id} \otimes \varphi) \circ \delta, \\
& \psi_{*}=(\mathrm{id} \otimes \psi) \circ \widehat{\delta} .
\end{aligned}
$$

One can easily verify that $\varphi_{*}$ and $\psi_{*}$ are completely bounded. Consequently $\psi_{*} \otimes$ id and id $\otimes \varphi_{*}$ are bounded linear mappings acting on $B=\widehat{A} \otimes A$. 
Proposition 8.4. For any $\psi \in \widehat{A}^{*}, \varphi \in A^{*}$ we have:

$$
\begin{aligned}
(\mathrm{id} \otimes \mathrm{id} \otimes \psi \otimes \varphi) W^{B} & =U[(\mathrm{id} \otimes \psi) \widehat{W} \otimes 1] U^{*}[1 \otimes(\mathrm{id} \otimes \varphi) W], \\
(\mathrm{id} \otimes \mathrm{id} \otimes \psi \otimes \varphi) \circ \delta^{B} & =\operatorname{Ad}_{W^{o}}\left(\psi_{*} \otimes \mathrm{id}\right) \circ \mathrm{Ad}_{W^{*}}\left(\mathrm{id} \otimes \varphi_{*}\right) .
\end{aligned}
$$

Proof. The first formula follows immediately from (8.9). Using the second formula of (8.1) we compute:

$$
\begin{aligned}
\left(\sigma_{W} \otimes \mathrm{id} \otimes \mathrm{id}\right) \circ \delta^{B} & =\left(\sigma_{W} \otimes \mathrm{id} \otimes \mathrm{id}\right) \circ\left(\mathrm{id} \otimes \sigma_{W} \otimes \mathrm{id}\right) \circ(\widehat{\delta} \otimes \delta) \\
& =(\mathrm{id} \otimes \widehat{\delta} \otimes \mathrm{id}) \circ\left(\sigma_{W} \otimes \mathrm{id}\right) \circ(\mathrm{id} \otimes \delta) .
\end{aligned}
$$

Therefore

$$
\delta^{B}=\left(\sigma_{W}^{-1} \otimes \mathrm{id} \otimes \mathrm{id}\right) \circ(\mathrm{id} \otimes \widehat{\delta} \otimes \mathrm{id}) \circ\left(\sigma_{W} \otimes \mathrm{id}\right) \circ(\mathrm{id} \otimes \delta)
$$

and

$$
(\mathrm{id} \otimes \mathrm{id} \otimes \psi \otimes \varphi) \circ \delta^{B}=\sigma_{W}^{-1} \mathrm{\circ}\left(\mathrm{id} \otimes \psi_{*}\right) \circ \sigma_{W^{\mathrm{o}}}\left(\mathrm{id} \otimes \varphi_{*}\right) .
$$

Remembering that $\sigma_{W}=\sigma \circ \mathrm{Ad}_{W^{*}}$ and using the relation $\sigma \circ\left(\mathrm{id} \otimes \psi_{*}\right) \circ \sigma=\psi_{*} \otimes \mathrm{id}$ we get the second formula of (8.11).

\section{Proposition 8.5.}

1. For any $\varphi \in A_{*}$ and any $c \in \mathcal{D}\left(\eta^{B}\right)$ we have: $\left(\operatorname{id} \otimes \varphi_{*}\right) c \in \mathcal{D}\left(\eta^{B}\right)$ and

$$
\eta^{B}\left(\left(\mathrm{id} \otimes \varphi_{*}\right) c\right)=[1 \otimes(\mathrm{id} \otimes \varphi) W] \eta^{B}(c) .
$$

2. For any $\psi \in \widehat{A}_{*}$ and any $c \in \mathcal{D}\left(\eta_{d}^{B}\right)$ we have: $\left(\psi_{*} \otimes \mathrm{id}\right) c \in \mathcal{D}\left(\eta_{d}^{B}\right)$ and

$$
\eta_{d}^{B}\left(\left(\psi_{*} \otimes \mathrm{id}\right) c\right)=[(\mathrm{id} \otimes \psi) \widehat{W} \otimes 1] \eta_{d}^{B}(c) .
$$

3. For any $\mu \in B_{*}$ and any $c \in \mathcal{D}\left(\eta^{B}\right)$ we have: $(\mathrm{id} \otimes \mu) \delta^{B}(c) \in \mathcal{D}\left(\eta^{B}\right)$ and

$$
\eta^{B}\left((\mathrm{id} \otimes \mu) \delta^{B}(c)\right)=\left[(\operatorname{id} \otimes \mu) W^{B}\right] \eta^{B}(c) .
$$

In the last point, id denotes the identity map acting on operators in $\mathcal{H} \otimes \mathcal{H}$.

Proof. Ad 1. For any $\sigma$-weakly continuous functional $\varphi$, the mapping id $\otimes \varphi$ maps strongly convergent sequences into strongly convergent sequences. Formula $\delta(a)=W(1 \otimes a) W^{*}$ (cf. (3.7)) shows that the comultiplication $\delta$ has the same property. Therefore $\varphi_{*}$ maps strongly convergent sequences into strongly convergent sequences. Therefore we may assume that $c=b \otimes a$, where $b \in \mathcal{D}\left(\widehat{\eta}_{L}\right)$ and $a \in \mathcal{D}(\eta)$ (by definition $\mathcal{D}\left(\widehat{\eta}_{L}\right) \otimes_{\text {alg }} \mathcal{D}(\eta)$ is a core of $\eta^{B}=\widehat{\eta}_{L} \otimes \eta$ ). In this case our relation takes the form

$$
\left.\widehat{\eta}_{L}(b) \otimes \eta(\varphi * a)=\widehat{\eta}_{L}(b) \otimes[\operatorname{id} \otimes \varphi) W\right] \eta(a),
$$

which immediately reduces to (1.6) (cf. (3.6)).

Ad 2. In this point we may assume that $c=b \otimes a$, where $b \in \mathcal{D}(\widehat{\eta})$ and $a \in \mathcal{D}\left(\eta_{L}\right)$. In this case our relation takes the form

$$
\widehat{\eta}((\mathrm{id} \otimes \psi) \widehat{\delta}(b)) \otimes \eta_{L}(a)=[(\mathrm{id} \otimes \psi) \widehat{W}] \widehat{\eta}(b) \otimes \eta_{L}(a),
$$

which immediately reduces to (7.2).

Ad 3. By Remark 3.10 we may assume that $\mu=\psi \otimes \varphi$, where $\psi \in \widehat{A}^{*}$ and $\varphi \in A^{*}$. (the reader should notice that $\widehat{A}_{*} \otimes_{\mathrm{alg}} A_{*}$ is norm dense in $\left.B_{*}\right)$. Let $c \in \mathcal{D}\left(\eta^{B}\right)$. Using Statement 1 , Proposition 8.2. Statement 2 and again Proposition 8.2 we obtain: $\left(\operatorname{id} \otimes \varphi_{*}\right) c \in \mathcal{D}\left(\eta^{B}\right), \operatorname{Ad}_{W^{*}}\left(\operatorname{id} \otimes \varphi_{*}\right) c \in \mathcal{D}\left(\eta_{d}^{B}\right)$, $\left(\psi_{*} \otimes \mathrm{id}\right) \operatorname{Ad}_{W^{*}}\left(\mathrm{id} \otimes \varphi_{*}\right) c \in \mathcal{D}\left(\eta_{d}^{B}\right)$ and

$$
\operatorname{Ad}_{W}\left(\psi_{*} \otimes \mathrm{id}\right) \operatorname{Ad}_{W^{*}}\left(\mathrm{id} \otimes \varphi_{*}\right) c \in \mathcal{D}\left(\eta^{B}\right) .
$$


According to (8.11) the last expression coincides with (id $\otimes \mu) \delta^{B}(c)$. Taking into account (8.11) and 8.5 we have:

$$
\begin{aligned}
{\left[(\mathrm{id} \otimes \mu) W^{B}\right] \eta^{B}(c) } & =U[(\mathrm{id} \otimes \psi) \widehat{W} \otimes 1] U^{*}[1 \otimes(\mathrm{id} \otimes \varphi) W] \eta^{B}(c) \\
& =U[(\mathrm{id} \otimes \psi) \widehat{W} \otimes 1] U^{*} \eta^{B}\left(\left(\mathrm{id} \otimes \varphi_{*}\right) c\right) \\
& =\lambda^{i / 4} U[(\mathrm{id} \otimes \psi) \widehat{W} \otimes 1] \eta_{d}^{B}\left(\operatorname{Ad}_{W^{*}}\left(\mathrm{id} \otimes \varphi_{*}\right) c\right) \\
& =\lambda^{i / 4} U \eta_{d}^{B}\left(\left(\psi_{*} \otimes \mathrm{id}\right) \operatorname{Ad}_{W^{*}}\left(\mathrm{id} \otimes \varphi_{*}\right) c\right) \\
& =\eta^{B}\left(\operatorname{Ad}_{W}\left(\psi_{*} \otimes \mathrm{id}\right) \operatorname{Ad}_{W^{*}}\left(\mathrm{id} \otimes \varphi_{*}\right) c\right) \\
& =\eta^{B}\left((\mathrm{id} \otimes \mu) \delta^{B}(c)\right)
\end{aligned}
$$

Let $\kappa^{B}=R^{B} \circ \tau_{i / 2}^{B}$. Then $\kappa^{B}=(\widehat{\kappa} \otimes \kappa) \circ \mathrm{Ad}_{W^{*}}$. Let $\psi \in \widehat{A}^{*}$ and $\varphi \in A^{*}$ be linear functionals such that $\psi \circ \widehat{\kappa} \in \widehat{A}^{*}$ and $\varphi \circ \kappa \in A^{*}$. For any $c \in B$ we set:

$$
\mu(c)=(\psi \otimes \varphi)\left(W^{*} c W\right)
$$

Then $\mu \in B^{*}$ and one can easily verify that

In particular $\mu \circ \kappa^{B} \in B^{*}$.

$$
\mu \circ \kappa^{B}=\psi \circ \widehat{\kappa} \otimes \varphi \circ \kappa
$$

Proposition 8.6. For any $\mu \in B^{*}$ such that $\mu^{\circ} \kappa^{B} \in B^{*}$ we have:

$$
\left(\mathrm{id} \otimes \mu^{\circ} \kappa^{B}\right) W^{B}=(\mathrm{id} \otimes \mu)\left(W^{B^{*}}\right) .
$$

Proof. In the following computation we use leg numbering notation for operators acting on $\mathcal{H}^{\otimes 4}=$ $\mathcal{H} \otimes \mathcal{H} \otimes \mathcal{H} \otimes \mathcal{H}$. Our first aim is formula (8.16). We start with the pentagonal equation $W_{14}^{*} W_{13}^{*}=$ $W_{34} W_{13}^{*} W_{34}^{*}$. Multiplying the both sides from the right by $W_{12}^{*}$ we get

$$
W_{14}^{*} W_{13}^{*} W_{12}^{*}=W_{34} W_{13}^{*} W_{12}^{*} W_{34}^{*} .
$$

Combining this formula with the pentagonal equation $W_{13}^{*} W_{12}^{*}=W_{23} W_{12}^{*} W_{23}^{*}$ we obtain:

$$
W_{14}^{*} W_{23} W_{12}^{*} W_{23}^{*}=W_{34} W_{23} W_{12}^{*} W_{23}^{*} W_{34}^{*} .
$$

For any operator $V$ acting on $\mathcal{H}^{\otimes 4}$ we set:

$$
\mathcal{A} d(V)=(J \otimes \widehat{J} \otimes J \otimes \widehat{J}) V(J \otimes \widehat{J} \otimes J \otimes \widehat{J})
$$

Clearly $\mathcal{A} d$ is an conjugate linear multiplicative operation acting on $\mathcal{L}\left(\mathcal{H}^{\otimes 4}\right)$. Using (5.22) we obtain immediately the relations: $\mathcal{A} d\left(W_{34}\right)=W_{34}^{*}, \mathcal{A} d\left(W_{14}^{*}\right)=W_{14}$ and $\mathcal{A} d\left(W_{12}^{*}\right)=W_{12}$. Furthermore, taking into account definition (8.4) we see that $\mathcal{A} d\left(W_{23}\right)=U_{23} W_{23}^{*}$. Applying $\mathcal{A} d$ to the both sides of 8.14) we get:

$$
W_{14} U_{23} W_{23}^{*} W_{12} W_{23} U_{23}^{*}=W_{34}^{*} U_{23} W_{23}^{*} W_{12} W_{23} U_{23}^{*} W_{34} .
$$

Multiplying both sides by $W_{13}$ from the right and using pentagon equation $W_{34} W_{13}=W_{13} W_{14} W_{34}$ we obtain

$$
W_{14} U_{23} W_{23}^{*} W_{12} W_{23} U_{23}^{*} W_{13}=W_{34}^{*} U_{23} W_{23}^{*} W_{12} W_{23} U_{23}^{*} W_{13} W_{14} W_{34} .
$$

The reader should notice that the 'third leg' of $W_{23} U_{23}^{*}=\left\{(\widehat{J} \otimes J) W^{*}(\widehat{J} \otimes J)\right\}_{23}$ 'belongs' to the commutant $A^{\prime}$ of $A$, whereas $W_{13} \in M(\widehat{A} \otimes 1 \otimes A \otimes 1)$. Therefore $W_{23} U_{23}$ commutes with $W_{13}$ and

$$
W_{23}^{*} W_{12} W_{23} U_{23}^{*} W_{13}=W_{23}^{*} W_{12} W_{13} W_{23} U_{23}^{*}=W_{12} U_{23}^{*} .
$$

Therefore (8.15) takes the form

$$
W_{14} U_{23} W_{12} U_{23}^{*}=W_{34}^{*} U_{23} W_{12} U_{23}^{*} W_{14} W_{34} .
$$

Now we perform the cyclic permutation of the first three $\mathcal{H}$ in $\mathcal{H}^{\otimes 4}=\mathcal{H} \otimes \mathcal{H} \otimes \mathcal{H} \otimes \mathcal{H}$. It results with the following replacement of the 'leg numbers': $1 \rightarrow 3 \rightarrow 2 \rightarrow 1$. Then $W_{12}$ goes into $W_{31}=\widehat{W}_{13}^{*}$ and our formula takes the form

$$
W_{34} U_{12} \widehat{W}_{13}^{*} U_{12}^{*}=W_{24}^{*} U_{12} \widehat{W}_{13}^{*} U_{12}^{*} W_{34} W_{24} .
$$

Rearranging this formula we obtain

$$
W^{B^{*}}=W_{24}^{*} U_{12} \widehat{W}_{13}^{*} U_{12}^{*}=W_{34} U_{12} \widehat{W}_{13}^{*} U_{12}^{*} W_{24}^{*} W_{34}^{*} .
$$


Let $\mu$ be the functional on $B$ introduced by (8.12). Applying id $\otimes \mu$ to the both sides of (8.16) and using in the third step formulae (3.13) and (7.3) we obtain:

$$
\begin{aligned}
(\mathrm{id} \otimes \mu) W^{B^{*}} & =(\mathrm{id} \otimes \mathrm{id} \otimes \psi \otimes \varphi)\left(U_{12} \widehat{W}_{13}^{*} U_{12}^{*} W_{24}^{*}\right) \\
& =U\left[(\mathrm{id} \otimes \psi)\left(\widehat{W}^{*}\right) \otimes 1\right] U^{*}\left[1 \otimes(\mathrm{id} \otimes \varphi)\left(W^{*}\right)\right] \\
& =U[(\mathrm{id} \otimes \psi \mathrm{\circ} \widehat{\kappa}) \widehat{W} \otimes 1] U^{*}[1 \otimes(\mathrm{id} \otimes \varphi \circ \kappa) W] \\
& =(\mathrm{id} \otimes \mathrm{id} \otimes \psi \circ \widehat{\kappa} \otimes \varphi \circ \kappa)\left(U_{12} \widehat{W}_{13} U_{12}^{*} W_{24}\right) \\
& =\left(\mathrm{id} \otimes \mu \circ \kappa^{B}\right)\left(W^{B}\right) .
\end{aligned}
$$

In this way we showed that (8.13) holds for any $\mu$ of the form (8.12). Clearly the set of functionals of the form 8.12) is linearly weakly* dense in $B^{*}$. Moreover for any $t \in \mathbb{R}$ the functional $\mu^{\circ} \tau_{t}^{B}$ is again of the form 8.12). Indeed, remembering that $W$ commutes with $Q \otimes Q$ we see that $\tau_{t}^{B}=\widehat{\tau}_{t} \otimes \tau_{t}$ commutes with $\operatorname{Ad}_{W^{*}}$ and

$$
\mu \circ \tau_{t}^{B}(c)=\left(\psi \circ \widehat{\tau}_{t} \otimes \varphi \circ \tau_{t}\right)\left(W^{*} c W\right) .
$$

Now, using Remark 3.11 we obtain (8.13) in full generality.

Let us summarize the results obtained so far. We have constructed the sequence of objects: $B, \delta^{B}, R^{B}, \tau^{B}, h^{B}, \eta^{B}, W^{B}$. Taking into account Proposition 8.1 Proposition 8.3 formula (8.10), Statement 3 of Proposition 8.5 and Proposition 8.6 we see that these objects satisfy all the assumptions of Theorem 3.9 Making profit of this theorem we obtain

Theorem 8.7. The pair $\left(B, \delta^{B}\right)$ is a unimodular weighted Hopf $C^{*}$-algebra with $\lambda=1$. The unitary antipode, the scaling group and the Haar weight related to $\left(B, \delta^{B}\right)$ are given by [8.7). The corresponding Kac-Takesaki operator coincides with (8.9).

The unimodularity of $\left(B, \delta^{B}\right)$ follows from 8.8 . It shows that the left and right Haar weight coincide. By the theory developed in Section $3, W^{B}$ is a manageable multiplicative unitary. Inserting definition (8.4) into (8.9) we obtain

$$
W^{B}=((\widehat{J} \otimes J) W(\widehat{J} \otimes J))_{12} W_{12} \widehat{W}_{13} W_{12}^{*}\left((\widehat{J} \otimes J) W^{*}(\widehat{J} \otimes J)\right)_{12} W_{24} .
$$

By pentagonal equation $W_{12} \widehat{W}_{13} W_{12}^{*}=\widehat{W}_{23} \widehat{W}_{13}$. Therefore

$$
W^{B}=((\widehat{J} \otimes J) W(\widehat{J} \otimes J))_{12} \widehat{W}_{23} \widehat{W}_{13}\left((\widehat{J} \otimes J) W^{*}(\widehat{J} \otimes J)\right)_{12} W_{24} .
$$

This formula admits further simplification:

$$
W^{B}=\widehat{W}_{23} \widehat{W}_{13}\left((J \otimes J) \widehat{W}^{*}(J \otimes J)\right)_{23} W_{24} .
$$

The verification of the last equality is left to the reader as an (un-easy) exercise.

\section{Appendix}

A. Multipliers and morphisms. In this Appendix, we collect some technical statements concerning the multipliers and the morphisms of the $C^{*}$-algebras.

For any $\varphi \in A^{*}$ and $a, b \in A$ we define functionals $b \varphi$ and $\varphi a$ in $A^{*}$ by

$$
(b \varphi)(a)=\varphi(a b)=(\varphi a)(b) .
$$

Then $A^{*}$ is a Banach $A$-bimodule.

Let $A$ be a $C^{*}$-algebra and $\left\{e_{\alpha}\right\}$ an approximate identity of $A$. Let $\varphi \in A^{*}$. Then $\varphi$ is written in the form $\varphi(a)=(x \mid \pi(a) y)$, where $\{\pi, \mathcal{H}\}$ is a representation of $A$ and $x, y \in \mathcal{H}$. For any $a \in A$ we have

$$
\left|\left(e_{\alpha} \varphi-\varphi\right)(a)\right|=\left|\varphi\left(a e_{\alpha}-a\right)\right|=\left|\left(x \mid \pi\left(a e_{\alpha}-a\right) y\right)\right| \leq\|x\|\|a\|\left\|\pi\left(e_{\alpha}\right) y-y\right\|
$$

and

$$
\left|\left(\varphi e_{\alpha}-\varphi\right)(a)\right|=\left|\varphi\left(e_{\alpha} a-a\right)\right|=\left|\left(x \mid \pi\left(e_{\alpha} a-a\right) y\right)\right| \leq\left\|\pi\left(e_{\alpha}\right) x-x\right\|\|a\|\|y\| .
$$

Hence the sets $\left\{\varphi e_{\alpha}\right\}$ and $\left\{e_{\alpha} \varphi\right\}$ converges in norm to $\varphi$. Thus we find that

$$
\overline{\left\{a \varphi: a \in A, \varphi \in A^{*}\right\}}=\overline{\left\{\varphi a: a \in A, \varphi \in A^{*}\right\}}=A^{*} .
$$

Now we recall the Doran-Wichmann's factorization theorem: 
Theorem A.1 ([5]). Let $B$ be a Banach algebra and $E$ a left (resp. right) Banach B-module. If $B$ has a left (resp. right) approximate identity bounded by $K \geq 1$, then for any $x \in E$ and for any $\varepsilon>0$ there exist $b \in B$ and $y \in E$ such that

$$
x=b y, \quad\|b\| \leq K, \quad y \in \overline{B x} \quad \text { (resp. } y \in \overline{x B}), \quad\|y-x\|<\varepsilon .
$$

In particular, $\overline{B E}=B E($ resp. $\overline{E B}=E B)$.

Combining this theorem with (A.1), we find that

Proposition A.2. Let $A$ be a $C^{*}$-algebra. Then $A^{*}=A A^{*}=A^{*} A$, i.e. for any $\varphi \in A^{*}$ there exist $\psi \in A^{*}$ and $a \in A$ such that $\varphi=a \psi($ or $\varphi=\psi a)$.

Lemma A.3. Let $A$ and $B$ be $C^{*}$-algebras and $\varphi \in B^{*}$. Then the slice mapping $\operatorname{id}_{A} \otimes \varphi: A \otimes B \rightarrow A$ has an extension $\left(\operatorname{id}_{A} \otimes \varphi\right)^{\sim}$ from $M(A \otimes B)$ to $M(A)$ such that $\left\|\left(\operatorname{id}_{A} \otimes \varphi\right)^{\sim}\right\|=\|\varphi\|$.

Proof. Let $\left\{c_{\alpha}\right\}$ be a net in $A \otimes B$ converging to zero with respect to the strict topology on $M(A \otimes B)$. By Proposition A.2 $\varphi$ is of the form $b \psi$ for some $b \in A$ and $\psi \in A^{*}$. Then, by the boundness of $\psi$, for any $a \in A,\left\{\left(\operatorname{id}_{A} \otimes \varphi\right)\left(c_{\alpha}\right)\right\} a=\left(\operatorname{id}_{A} \otimes \psi\right)\left(c_{\alpha}(a \otimes b)\right)$ converges in norm to zero. Similarly, we obtain that the net $\left\{a\left\{\left(\operatorname{id}_{A} \otimes \varphi\right)\left(c_{\alpha}\right)\right\}\right\}$ converges in norm to zero. This proves that the net $\left\{\left(\operatorname{id}_{A} \otimes \varphi\right)\left(c_{\alpha}\right)\right\}$ converges to zero with respect to the strict topology. Hence the slice mapping $\operatorname{id}_{A} \otimes \varphi: A \otimes B \rightarrow A$ has an extension to the mapping $\left(\operatorname{id}_{A} \otimes \varphi\right)^{\sim}$ from $M(A \otimes B)$ to $M(A)$.

It is known that the unit ball of a $C^{*}$-algebra is strictly dense in the unit ball of the multiplier algebra. Therefore the norm of $\left(\operatorname{id}_{A} \otimes \varphi\right)^{\sim}$ coincides with the norm of id $\otimes \varphi$. It is clear that norms id $\otimes \varphi$ and $\varphi$ are the same.

Throughout this paper, the notion of the morphism between the two $C^{*}$-algebras plays an important role. Here we recall the definition of the morphism.

Definition A.4 (31]). Let $A$ and $B$ be $C^{*}$-algebras. Then $\pi$ is said to be in the set $\operatorname{Mor}(A, B)$ of morphisms from $A$ to $B$ if $\pi$ is a homomorphism from the $C^{*}$-algebra $A$ to the multiplier algebra $M(B)$ such that the subset $\pi(A) B$ of $B$ is norm dense in $B$.

Each morphism $\pi$ of $A$ to $B$ can be extended uniquely to a morphism $\widetilde{\pi}$ of the multiplier algebra $M(A)$ to the multiplier algebra $M(B)$ :

$$
\widetilde{\pi}(m) \sum_{i} \pi\left(a_{i}\right) x_{i}=\sum_{i} \pi\left(m a_{i}\right) x_{i}
$$

for $m \in M(A), a_{i} \in A$ and $x_{i}$ in the carrier Hilbert space of $B$. In this paper this extension of $\pi$ is denoted by the same letter (we omit tilde).

The morphisms admit even further extensions to unbounded elements affiliated with $C^{*}$-algebras (see [30, 31]). If $\rho$ is a strictly positive self-adjoint operator affiliated with $A$, then $\left\{\rho^{i t}\right\}_{t \in \mathbb{R}}$ is a one parameter group of unitaries in $M(A)$ continuous with respect to the strict topology and $\pi(\rho)^{i t}=\pi\left(\rho^{i t}\right)$. In this formula $\pi(\rho)$ denotes the element affiliated with $B$ corresponding (via morphism $\pi \in \operatorname{Mor}(A, B))$ to $\rho$ affiliated with $A$.

B. GNS maps. This Appendix is devoted to the notion of GNS map. Neglecting certain details this notion is equivalent to the notion of generalized Hilbert algebra. In our opinion it is more convenient, when we have to deal with many different scalar products defined on the same operator algebra.

In the following definition operator algebra means either $\mathrm{C}^{*}$-algebra or von Neumann algebra of operators acting on a Hilbert space $\mathcal{H}$. In the $\mathrm{C}^{*}$-algebra case we shall assume that it acts on $\mathcal{H}$ in a non-degenerate way. Consequently in the case of von Neumann algebra we understand that it contains the identity operator $1_{\mathcal{H}}$.

Definition B.1. Let $B$ be an operator algebra acting on a Hilbert space $\mathcal{H}$ and $\eta$ be a (unbounded) linear mapping from $B$ into $\mathcal{H}$. We say that $\eta$ is a GNS map if the domain $\mathcal{D}(\eta)$ is a left ideal in $B$ and

$$
\eta(a b)=a \eta(b)
$$

for any $a \in B$ and $b \in \mathcal{D}(\eta)$. The GNS map $\eta$ is called closed if it is closed with respect to strong operator topology on $B$ and norm topology on $\mathcal{H}$. We say that a GNS map defined on a $C^{*}$-algebra is densely defined if its domain is norm dense in the $C^{*}$-algebra. Similarly a GNS map defined 
on a von Neumann algebra is said to be densely defined if its domain is strongly dense in the von Neumann algebra.

The reader should notice that using the term 'densely defined' we refer to different topologies in $\mathrm{C}^{*}$-algebra and von Neumann algebra cases. On the contrary to define closedness we use in both cases the same strong operator topology on $B$. The same topology is used when we speak about continuous GNS maps, closable GNS maps, the closure of a GNS map and a core (essential domain) of a GNS map.

Let $B$ be an operator algebra acting on a Hilbert space $\mathcal{H}$ and $\Omega \in \mathcal{H}$. For any $b \in B$ we set

$$
\eta(b)=b \Omega \text {. }
$$

Then $\eta$ is a continuous GNS map with $\mathcal{D}(\eta)=B$. To obtain less trivial example assume that $\mathcal{H}$ is a direct orthogonal sum of a family $\left\{\mathcal{H}_{i}\right\}_{i \in I}$ of $B$-invariant subspaces and for each $i \in I$ pick up a vector $\Omega_{i} \in \mathcal{H}_{i}$. Then the formula

$$
\eta(b)=\sum_{i \in I} b \Omega_{i}
$$

defines a closed GNS map. By definition $\mathcal{D}(\eta)$ is the set of all $b \in B$ for which the above series is norm convergent. It turns out (cf. Theorem B.7) that any closed GNS map defined on a von Neumann algebra acting on a separable Hilbert space is of the form (B.2) with a denumerable index set $I$.

Now we introduce the notion of the commutant $\eta^{\prime}$ of a GNS-map $\eta$. Let $B$ be an operator algebra acting on a Hilbert space $\mathcal{H}, \eta$ be a GNS map defined on $B$ and $B^{\prime}$ be the commutant of $B$. Assume that the intersection of kernels of all $b \in \mathcal{D}(\eta)$ is trivial. This is always the case, when $\eta$ is densely defined. For any $c^{\prime} \in B^{\prime}$ and $x \in \mathcal{H}$ we set:

$$
\left(\begin{array}{c}
c^{\prime} \in \mathcal{D}\left(\eta^{\prime}\right) \\
\text { and } \\
x=\eta^{\prime}\left(c^{\prime}\right)
\end{array}\right) \Longleftrightarrow\left(\begin{array}{c}
c^{\prime} \eta(b)=b x \\
\text { for all } \\
b \in \mathcal{D}(\eta)
\end{array}\right) .
$$

The reader should notice that there is at most one vector $x$ satisfying the right hand side of the above equivalence. One can easily verify that $B^{\prime}$ is a von Neumann algebra and that $\eta^{\prime}$ is a closed GNS map defined on $B^{\prime}$. It turns out (cf. Theorem B.2) that $\eta^{\prime}$ is densely defined provided $\eta$ is closable.

In the following statements we use the double commutant $\eta^{\prime \prime}$ of a GNS map $\eta$. If $\eta$ is defined on an operator algebra $B$ then $\eta^{\prime \prime}$ is defined on the double commutant $B^{\prime \prime}$. By the famous von Neumann density theorem, $B^{\prime \prime}$ is the strong closure of $B$. We have the following

Theorem B.2. Let $B$ be an operator algebra acting on a Hilbert space $\mathcal{H}$ and $\eta$ be a closable GNS mapping densely defined on $B$. Then $\eta^{\prime}$ is densely defined on the von Neumann algebra $B^{\prime}, \eta^{\prime \prime}$ is an extension of $\eta$ and $\mathcal{D}(\eta)$ is a core of $\eta^{\prime \prime}$.

Proof. The inclusion $\eta \subset \eta^{\prime \prime}$ is obvious. Let $\bar{\eta}$ be the closure of $\eta^{\prime \prime}$ restricted to $\mathcal{D}(\eta), a \in B^{\prime \prime}$ and $x \in \mathcal{H}$. We have to show that

$$
\left(\begin{array}{c}
a \eta^{\prime}(p)=p x \\
\text { for all } \\
p \in \mathcal{D}\left(\eta^{\prime}\right)
\end{array}\right) \Longrightarrow\left(\begin{array}{c}
a \in \mathcal{D}(\bar{\eta}) \\
\text { and } \\
x=\bar{\eta}(a)
\end{array}\right) .
$$

The condition on the right hand side of (B.4) means that the pair $(a, x)$ belongs to the closure of the graph of $\eta$ in the (strong $\times$ norm)-topology. Remembering that the neighborhoods in strong topology are determined by finite sets of vectors it is sufficient to show that for any $\Omega_{1}, \Omega_{2}, \ldots, \Omega_{n} \in$ $\mathcal{H}$ and

$$
\begin{aligned}
& \text { for any } \varepsilon>0 \text { there exists } b \in \mathcal{D}(\eta) \text { such that } \\
& \qquad\|x-\eta(b)\|^{2}+\sum_{k=1}^{n}\left\|a \Omega_{k}-b \Omega_{k}\right\|^{2}<\varepsilon^{2} .
\end{aligned}
$$

Let $\widetilde{\mathcal{H}}$ be the direct sum of $1+n$ copies of $\mathcal{H}$. The copies will be labelled by numbers $0,1,2, \ldots, n$. For any $k=0,1, \ldots, n$ we denote by $x_{k} \in \mathcal{H}$ the $k$-th component of a vector $x \in \widetilde{\mathcal{H}}$. Consequently for any $b \in \mathcal{L}(\widetilde{\mathcal{H}})$, the matrix elements of $b$ will be denoted by $b_{k l} \in \mathcal{L}(\mathcal{H})(k, l=0,1,2, \ldots, n)$. In what follows we shall use the diagonal action of $B$ on $\widetilde{\mathcal{H}}$ : any $b \in B$ defines an operator $\tilde{b} \in \mathcal{L}(\widetilde{\mathcal{H}})$ with matrix elements $\tilde{b}_{k l}=b \delta_{k l}(k, l=0,1,2, \ldots, n)$. 
For any $b \in \mathcal{D}(\eta)$ we denote by $\tilde{\eta}(b)$ the element of $\widetilde{\mathcal{H}}$ such that

$$
\tilde{\eta}(b)_{k}= \begin{cases}\eta(b) & \text { for } k=0 \\ b \Omega_{k} & \text { for } k=1,2, \ldots, n .\end{cases}
$$

We shall also use the vector $\tilde{x} \in \widetilde{\mathcal{H}}$ such that

$$
\tilde{x}_{k}=\left\{\begin{array}{cl}
x & \text { for } k=0 \\
a \Omega_{k} & \text { for } k=1,2, \ldots, n .
\end{array}\right.
$$

With this notation, (B.5) may be rewritten in the following equivalent form:

$$
\tilde{x} \in\{\tilde{\eta}(b): b \in B\}^{\text {closure }}
$$

Let $p \in \mathcal{L}(\widetilde{\mathcal{H}})$ be the orthogonal projection onto orthogonal complement of $\{\tilde{\eta}(b): b \in B\}$. One can easily show that the latter space is invariant under the diagonal action of $B$. Therefore the matrix elements of $p$ belongs to the commutant of $B: p_{k l} \in B^{\prime}$ for all $k, l=0,1,2, \ldots, n$. By definition $p \tilde{\eta}(b)=0$. It means that

$$
p_{k 0} \eta(b)+\sum_{l=1}^{n} p_{k l} b \Omega_{l}=0
$$

and

$$
p_{k 0} \eta(b)=-b \sum_{l=1}^{n} p_{k l} \Omega_{l}
$$

for $k=0,1,2, \ldots, n$. This relation holds for all $b \in \mathcal{D}(\eta)$. Definition (B.3) shows now that $p_{k 0} \in \mathcal{D}\left(\eta^{\prime}\right)$ and $\eta^{\prime}\left(p_{k 0}\right)=-\sum_{l=1}^{n} p_{k l} \Omega_{l}$.

Assume now that the condition on the left hand side of $(\underline{B .4})$ is satisfied. Then

$$
p_{k 0} x=a \eta^{\prime}\left(p_{k 0}\right)=-a \sum_{l=1}^{n} p_{k l} \Omega_{l}
$$

and

$$
p_{k 0} x+\sum_{l=1}^{n} p_{k l} a \Omega_{l}=0
$$

This relation holds for $k=0,1,2, \ldots, n$. It shows that $p \tilde{x}=0$ and (B.6) follows. This way the implication (B.4) is proved.

Setting $a=0$ we see that $x=0$ is the only vector killed by all $p \in \mathcal{D}\left(\eta^{\prime}\right)$. Remembering that $\mathcal{D}\left(\eta^{\prime}\right)$ is a left ideal in $B^{\prime}$ and using [4, Chapitre I, $\S 3$, Corollaire 3] we see that $\mathcal{D}\left(\eta^{\prime}\right)$ is dense in $B^{\prime}$. Comparing now (B.4) with (B.3) we see that $\bar{\eta}$ is an extension of $\eta^{\prime \prime}: \eta^{\prime \prime} \subset \bar{\eta}$. The converse inclusion is obvious: $\eta^{\prime \prime}$ is an extension of $\eta$ and $\eta^{\prime \prime}$ is closed. Hence $\bar{\eta} \subset \eta^{\prime \prime}$.

Assume now that $\mathcal{H}$ is separable. Our nearest aim is to show that any closed GNS map is of the form (B.2). We start with the following

Proposition B.3. Let $M$ a von Neumann algebra acting on a separable Hilbert space $\mathcal{H}$ and $\eta$ be a closed GNS-mapping on $M$. Then there exists $d \in \mathcal{D}(\eta)$ such that $M d$ is a core for $\eta$. One may choose $d$ in such a way that $d \geq 0$ and $\operatorname{Sp}(d) \subset\left\{1,2^{-1}, 2^{-2}, \ldots, 0\right\}$.

Proof.At first we shall prove that there exists elements $c_{n} \in \mathcal{D}(\eta)(n=1,2, \ldots)$ such that the set

$$
\left\{c_{n}: n=1,2, \ldots\right\}^{\text {linear span }}
$$

is a core for $\eta$. We know that $\eta$ is closed with respect to the strong topology on $M$ and norm topology on $\mathcal{H}$. We shall use the graph topology on $\mathcal{D}(\eta)$. Let

$$
K=\{a \in \mathcal{D}(\eta):\|a\| \leq 1,\|\eta(a)\| \leq 1\} .
$$

Clearly, $K$ is a closed subset of $\mathcal{D}(\eta)$. Choosing an orthonormal basis $\left\{e_{n}\right\}_{n \in \mathbb{N}}$ in $\mathcal{H}$ and setting

$$
d(a, b)^{2}=\sum_{n=1}^{\infty} \frac{1}{4^{n}}\left\|(a-b) e_{n}\right\|^{2}+\|\eta(a-b)\|^{2},
$$


we define a metric $d$ on $K$. One can easily show that the topology induced by this metric coincides with the graph topology on $\mathcal{D}(\eta)$ restricted to $K$. Moreover, the mapping

$$
K \ni a \longmapsto\left(\sum_{n=1}^{\infty} \oplus \frac{1}{2^{n}} a e_{n}\right) \oplus \eta(a) \in\left(\sum_{n=1}^{\infty} \oplus \mathcal{H}\right) \oplus \mathcal{H}
$$

is an isometric embedding and the target Hilbert space is separable. Therefore there exists a denumerable subset dense in $K$. The linear span of this subset is the core for $\eta$ of the form (B.7).

Multiplying if necessary by a decreasing sequence of positive numbers we may assume that $\sum\left\|c_{n}\right\|<\infty$ and $\sum\left\|\eta\left(c_{n}\right)\right\|<\infty$. Using sequence $\left\{c_{n}\right\}_{n=1,2, \ldots}$, we define a bounded linear mapping $c$

$$
\mathcal{H} \ni x \longmapsto c x=\sum_{n=1}^{\infty} c_{n} x .
$$

Then $c^{*} c=\sum_{n=1}^{\infty} c_{n}^{*} c_{n} \in M$. Let $d=\sqrt{c^{*} c}$ and $c=u d$ be the polar decomposition of $c$. Then $d \in M$ and the isometry $u$ is of the form

$$
\mathcal{H} \ni x \longmapsto u x=\sum_{n=1}^{\infty} u_{n} x \in \sum_{n=1}^{\infty}{ }^{\oplus} \mathcal{H}
$$

where all $u_{n} \in M$ and $\left\|u_{n}\right\| \leq 1$. Relation $c=u d$ means that

$$
c_{n}=u_{n} d
$$

for all $n=1,2, \ldots$, whereas relation $d=u^{*} c$ is equivalent to

$$
d=\sum_{n=1}^{\infty} u_{n}^{*} c_{n}
$$

The above series is norm converging. Also the series $\sum u_{n}^{*} \eta\left(c_{n}\right)$ is norm converging. Remembering that $\eta$ is closed we conclude that $d \in \mathcal{D}(\eta)$ and $M d \subset \mathcal{D}(\eta)(\mathcal{D}(\eta)$ is a left ideal!). Formula (B.8) shows now that the set (B.7) is contained in $M d$. Therefore $M d$ is a core for $\eta$.

The element $d$ constructed above is self-adjoint and positive. We may assume that $\|d\|<1$. Let $f$ be a simple function on $[0,1)$ defined by $f(0)=0$ and

$$
f(t)=\frac{1}{2^{n+1}} \quad \text { for } \quad t \in\left[\frac{1}{2^{n+1}}, \frac{1}{2^{n}}\right) .
$$

for $n=0,1,2, \ldots$. Then $t / 2 \leq f(t) \leq t$ for $t \in[0,1]$. Using this estimate one can easily show that $d$ and $f(d)$ generate the same left ideal in $M: M d=M f(d)$. Moreover $f(d) \geq 0$ and $\operatorname{Sp} f(d) \subset\left\{1,2^{-1}, 2^{-2}, \ldots, 0\right\}$. This way the last Statement of our Proposition is shown.

Definition B.4. Let $B$ be an operator algebra acting on a separable Hilbert space $\mathcal{H}$ and $\eta$ be a closed densely defined GNS-mapping on B. Assume that $\left\{\Omega_{n}\right\}_{n=1,2, \ldots}$ is a sequence of elements of $\mathcal{H}$ such that $B \Omega_{n} \perp B \Omega_{m}$ for $n \neq m$ and

$$
\eta(b)=\sum_{n}^{\infty} b \Omega_{n} .
$$

for any $b \in \mathcal{D}(\eta)$. Then we say that $(\underline{\mathrm{B} .9})$ is a vector presentation of the GNS mapping $\eta$.

Proposition B.5. Let (B.9) be a vector presentation of a GNS map $\eta$ of a closed densely defined GNS map $\eta$. Then for any $b \in \mathcal{D}\left(\eta^{\prime \prime}\right)$ we have

$$
\eta^{\prime \prime}(b)=\sum_{n=1}^{\infty} b \Omega_{n} .
$$

Proof. Let $P_{n}$ be the orthogonal projection onto $\overline{B \Omega_{n}}(n=1,2, \ldots)$. Then $P_{n}(n=1,2, \ldots)$ are pairwise orthogonal projections and denoting by $P_{0}$ the projection onto the orthogonal complement of $\sum^{\oplus} \overline{B \Omega_{n}}$ we obtain the decomposition of unity:

$$
1=\sum_{n=0}^{\infty} P_{n}
$$


Clearly all $P_{n}$ belong to $B^{\prime}$. For $n=1,2, \ldots$ we have

$$
P_{n} \eta(b)=P_{n} \sum_{n=1}^{\infty} b \Omega_{n}=b \Omega_{n}
$$

for all $b \in B$. It shows that $P_{n} \in \mathcal{D}\left(\eta^{\prime}\right)$ and $\eta^{\prime}\left(P_{n}\right)=\Omega_{n}$. Moreover $P_{0} \eta(b)=0$ for all $b \in B$. Therefore $P_{0} \in \mathcal{D}\left(\eta^{\prime}\right.$ and $\eta^{\prime}\left(P_{0}\right)=0$.

Assume that $b \in \mathcal{D}\left(\eta^{\prime \prime}\right)$. Then for any $c \in \mathcal{D}\left(\eta^{\prime}\right)$ we have $c \eta^{\prime \prime}(b)=b \eta^{\prime}(c)$. Setting $c=P_{n}$ we obtain $P_{n} \eta^{\prime \prime}(b)=b \eta^{\prime}\left(P_{n}\right)=b \Omega_{n}$ for $n=1,2, \ldots$ and $P_{0} \eta^{\prime \prime}(b)=b \eta^{\prime}\left(P_{0}\right)=0$ for $n=0$. Summing over $n$ we get (B.10).

Definition B.6. A vector presentation (B.9) is said to be exact if $\mathcal{D}\left(\eta^{\prime \prime}\right)$ coincides with the set of all elements $b \in B^{\prime \prime}$ such that the sum (B.10) is convergent.

We know that any bounded GNS-map $\eta$ is of the form $\eta(a)=a \Omega$, where $\Omega \in \mathcal{H}$. We shall use the Proposition B.3 to prove the following nice generalization of this fact.

Theorem B.7. Let $B$ be an operator algebra acting on a separable Hilbert space $\mathcal{H}$ and $\eta$ be a closed densely defined GNS-mapping on B. Then $\eta$ admits an exact vector presentation.

Proof. Let $\eta^{\prime}$ be the commutant of $\eta$. By Proposition B.3 there exists $d \in \mathcal{D}\left(\eta^{\prime}\right)$ such that $B^{\prime} d$ is a core for $\eta^{\prime}$. Assume that a vector $x \in \operatorname{ker} d$. Then $c d x=0$ for any $c \in B^{\prime}$. Therefore $x$ is killed by all elements of $\mathcal{D}\left(\eta^{\prime}\right)$. Remembering that $\mathcal{D}\left(\eta^{\prime}\right)$ is strongly dense in $B^{\prime}$ we see that $x=0$. This way we have shown that ker $d=\{0\}$.

By the last sentence of Proposition B.3 we may assume that $d$ is a self-adjoint positive operator with spectrum contained in $\left\{1,2^{-1}, 2^{-2}, \ldots, 0\right\}$. Then

$$
d=\sum_{n=1}^{\infty} 2^{-n} P_{n}
$$

where $P_{n} \in B^{\prime}$ are orthogonal projections such that $P_{n} P_{m}=0$ for $n \neq m$. Remembering that the kernel of $d$ is trivial we obtain

$$
\sum_{n=1}^{\infty} P_{n}=I
$$

For any $n=1,2, \ldots$, we set:

$$
\Omega_{n}=2^{n} P_{n} \eta^{\prime}(d)
$$

Then $d \Omega_{n}=P_{n} \eta^{\prime}(d)$ and

$$
\sum_{n=1}^{\infty} d \Omega_{n}=\sum_{n=1}^{\infty} P_{n} \eta^{\prime}(d)=\eta^{\prime}(d) .
$$

The reader should notice that the above series is norm converging. Since $P_{n} \in B^{\prime}$, the subspace $P_{n} \mathcal{H}$ is $B$-invariant and $B \Omega_{n} \subset P_{n} \mathcal{H}$. Remembering that $P_{n} P_{m}=0$ we see that $B \Omega_{n} \perp B \Omega_{m}$ for $n \neq m$. Formula $P_{n}=2^{n} P_{n} d$ shows that $P_{n} \in \mathcal{D}\left(\eta^{\prime}\right)$ and $\eta^{\prime}\left(P_{n}\right)=2^{n} P_{n} \eta^{\prime}(d)=\Omega_{n}$ for all natural $n$.

Let $b \in \mathcal{D}(\eta)$. Then, for any natural $n, P_{n} \eta(b)=b \eta^{\prime}\left(P_{n}\right)=b \Omega_{n}$. Therefore

$$
\eta(b)=\sum_{n=1}^{\infty} P_{n} \eta(b)=\sum_{n=1}^{\infty} b \Omega_{n} .
$$

Clearly the above formula is a vector presentation of $\eta$. If the series

$$
x=\sum_{n=1}^{\infty} b \Omega_{n}
$$

is converging for some $b \in B^{\prime \prime}$, then, using (B.11) we obtain:

$$
d x=\sum_{n=1}^{\infty} d b \Omega_{n}=\sum_{n=1}^{\infty} b d \Omega_{n}=b \sum_{n=1}^{\infty} d \Omega_{n}=b \eta^{\prime}(d) .
$$

Therefore for any $c \in B^{\prime}, c d x=c b \eta^{\prime}(d)=b c \eta^{\prime}(d)=b \eta^{\prime}(c d)$. Remembering that $B^{\prime} d$ is a core for $\eta^{\prime}$ we see that $c x=b \eta^{\prime}(c)$ for all $c \in \mathcal{D}\left(\eta^{\prime}\right)$. Hence $b \in \mathcal{D}\left(\eta^{\prime \prime}\right)$. It shows that the vector presentation constructed above is exact. 
Let us also note the following interesting

Proposition B.8. Let $B$ an operator algebra acting on a separable Hilbert space $\mathcal{H}$, $\eta$ be a closed densely defined GNS mapping on $B$ with a vector presentation

$$
\eta(b)=\sum_{n} b \Omega_{n}
$$

and $P_{n} \in \mathcal{D}\left(\eta^{\prime}\right)$ be the orthogonal projection onto $\overline{B \Omega_{n}}, n=1,2, \ldots$ (see the proof of Proposition B.5). Then the following statements are equivalent:

(1) The vector presentation (B.12) is exact.

(2) The left ideal of $B^{\prime}$ generated by $\left\{P_{1}, P_{2}, \ldots\right\}$ is a core for $\eta^{\prime}$.

Proof. Ad $2 \Rightarrow 1$.

Let $b$ be an element of $B^{\prime \prime}$. If the series $\sum_{k} b \Omega_{k}$ is convergent, then for any $a \in B^{\prime}$ and any $n=1,2, \ldots$ we have:

$$
b \eta^{\prime}\left(a P_{n}\right)=a b \eta^{\prime}\left(P_{n}\right)=a b \Omega_{n}=a P_{n} \sum_{k} b \Omega_{k} .
$$

If the left ideal in $B^{\prime}$ generated by $\left\{P_{1}, P_{2}, \ldots\right\}$ is a core for $\eta^{\prime}$ then $b \eta^{\prime}(a)=a \sum_{k} b \Omega_{k}$ for all $a \in \mathcal{D}(\eta)$. Hence $b \in \mathcal{D}\left(\eta^{\prime \prime}\right)$ and $\eta^{\prime \prime}(b)=\sum_{k} b \Omega_{k}$. It shows that the vector presentation (B.12) is exact.

Ad $1 \Rightarrow 2$.

Assume now that the left ideal $I$ of $B^{\prime}$ generated by $\left\{P_{1}, P_{2}, \ldots\right\}$ is not a core for $\eta^{\prime}$. Let $\theta$ be the closure of $\eta^{\prime}$ restricted to $I$. Then $\theta: B^{\prime} \rightarrow \mathcal{H}$ is a GNS mapping, $\theta \subset \eta^{\prime}$ and $\theta \neq \eta^{\prime}$. Therefore $\eta^{\prime \prime} \subset \theta^{\prime}$ and $\eta^{\prime \prime} \neq \theta^{\prime}$. Hence there exists $b \in \mathcal{D}\left(\theta^{\prime}\right)$ such that $b \notin \mathcal{D}\left(\eta^{\prime \prime}\right)$. Clearly $P_{n} \in \mathcal{D}(\theta)$ and $\theta\left(P_{n}\right)=\eta^{\prime}\left(P_{n}\right)=\Omega_{n}$. Now we have

$$
b \Omega_{n}=b \theta\left(P_{n}\right)=P_{n} \theta^{\prime}(b)
$$

and the series $\sum b \Omega_{n}=\sum_{n} P_{n} \theta^{\prime}(b)$ is convergent. Remember that $b \notin \mathcal{D}\left(\eta^{\prime \prime}\right)$ we see that the vector presentation (B.12) is not exact.

C. Weights on $\mathbf{C}^{*}$-algebras. In this Appendix, we collect some basic properties of weights on a separable $C^{*}$-algebras and their relations with GNS mappings. Let $h$ be a weight on a $C^{*}$-algebra $A$. We shall use the standard notation:

$$
\begin{aligned}
\mathfrak{N}_{h} & =\left\{a \in A: h\left(a^{*} a\right)<\infty\right\}, \\
\mathfrak{M}_{h} & =\left\{a \in A_{+}: h(a)<\infty\right\}^{\text {linear span }} \\
& =\left\{a^{*} b: a, b \in \mathfrak{N}_{h}\right\}^{\text {linear span }}
\end{aligned}
$$

Then $\mathfrak{N}_{h}$ is a left ideal and $\mathfrak{M}_{h}$ is a hereditary subalgebra in $A$. It is known that $h$ extends uniquely to a linear functional on $\mathfrak{M}_{h}$. We say that $h$ is locally finite if $\mathfrak{N}_{h}$ is dense in $A$.

By the GNS construction one can find a Hilbert space $\mathcal{H}$, a representation $\pi$ of $A$ acting on $\mathcal{H}$ and a GNS-map $\eta$ defined on the $\mathrm{C}^{*}$-algebra $B=\pi(A)$ such that

$$
\mathcal{D}(\eta)=\left\{\pi(b): b \in \mathfrak{N}_{h}\right\}
$$

the range of $\eta$ is dense in $\mathcal{H}$ and

$$
\|\eta(\pi(a))\|^{2}=h\left(a^{*} a\right)
$$

for all $a \in \mathfrak{N}_{h}$. We say that $(\mathcal{H}, \pi, \eta)$ is a GNS triple associated with the weight $h$. It is unique up to a unitary equivalence. If $h$ is finite then the GNS-map $\eta$ is bounded, defined on whole $\pi(A)$ and there exists a vector $\Omega \in \mathcal{H}$ such that $\eta(b)=b \Omega$ for all $b \in B$. In the general case we would like to have the map $\eta$ closed. This is the case if the weight $h$ is lower semicontinuous, i.e. if for any $t \in \mathbb{R}_{+}$the set $\left\{a \in A_{+}: h(a) \leq t\right\}$ is closed in $A$ in the sense of the norm topology. By a very important result of Haagerup [9], any lower semicontinuous weight $h$ on a $\mathrm{C}^{*}$-algebra $A$ is of the form

$$
h=\sum_{i \in I} h_{i}
$$

where $\left\{h_{i}\right\}_{i \in I}$ is a family of positive functionals on $A$. For separable $\mathrm{C}^{*}$-algebras the above sum is countable: 
Lemma C.1. Let $h$ be a locally finite lower semicontinuous weight on a separable $C^{*}$-algebra $A$. Then there exists a countable family $\left\{h_{i}\right\}_{i \in \mathbb{N}}$ of elements of $A_{+}^{*}$ such that

$$
h=\sum_{i \in \mathbb{N}} h_{i} .
$$

Proof. We start with formula (C.1). Replacing if necessary $h_{i}$ by the sum of $N$-copies of $h_{i} / N$, (where $N$ is a natural number larger than $\left\|h_{i}\right\|$ ) and removing zero-terms, we may assume that $\left\|h_{i}\right\| \leq 1$ and $h_{i} \neq 0$ for all $i \in I$. Let $\Lambda$ be the set $\left\{\psi \in A_{+}^{*}:\|\psi\| \leq 1\right\}$. Since $A$ is separable, the weak ${ }^{*}$ topology on the unit ball of $A^{*}$ is metrizable. Denote a metric by $d$. Put

$$
\begin{aligned}
& I_{1}=\left\{i \in I: d\left(h_{i}, 0\right) \in[1, \infty)\right\} \\
& I_{n}=\left\{i \in I: d\left(h_{i}, 0\right) \in\left[\frac{1}{n}, \frac{1}{n-1}\right)\right\}, n \geq 2 .
\end{aligned}
$$

It suffices to show that each $I_{n}$ is finite. Suppose that $I_{n}$ is infinite for some $n \in \mathbb{N}$. Since $\Lambda$ is weak ${ }^{*}$ compact, there exists a sequence $i_{m} \in I_{n}(m=1,2, \ldots)$ and an element $\psi \in \Lambda$ such that $h_{i_{m}} \longrightarrow \psi$ when $m \rightarrow \infty$. Clearly $d(\psi, 0) \geq n^{-1}$ and $\psi \neq 0$. For any $a \in \mathfrak{N}_{h}$ we have

$$
\sum_{m=1}^{\infty} h_{i_{m}}\left(a^{*} a\right) \leq \sum_{i \in I} h_{i}\left(a^{*} a\right)=h\left(a^{*} a\right)<\infty .
$$

It shows that $\psi\left(a^{*} a\right)=\lim _{m \rightarrow \infty} h_{i_{m}}\left(a^{*} a\right)=0$. Remembering that $\mathfrak{N}_{h}$ is norm-dense in $A$ we conclude that $\psi=0$. This contradiction shows that $I_{n}$ is finite for all $n \in \mathbb{N}$.

Theorem C.2. Let $h$ be a locally finite lower semicontinuous weight on a separable $C^{*}$-algebra $A$ and $(\mathcal{H}, \pi, \eta)$ be the GNS-triple associated with the weight $h$. Then $\mathcal{H}$ is separable and $\eta$ is closed.

Proof. We may assume that $h$ is given by (C.2). Let $\left(\mathcal{H}_{i}, \pi_{i}, \eta_{i}\right)$ be the GNS triple associated with $h_{i}$. In this case $\eta_{i}$ are continuous. Remembering that $A$ is separable and that the range of $\eta_{i}$ are dense in $\mathcal{H}_{i}$ we conclude that $\mathcal{H}_{i}$ are separable. By Lemma C.1 the index set $I$ is denumerable. Therefore the direct sum

$$
\widetilde{\mathcal{H}}=\sum_{i \in I}^{\oplus} \mathcal{H}_{i}
$$

is a separable Hilbert space. Using (C.2) one can easily show that

$$
\|\eta(\pi(a))\|^{2}=\sum_{i \in I} h_{i}\left(a^{*} a\right)=\left\|\sum_{i \in I}^{\oplus} \eta_{i}\left(\pi_{i}(a)\right)\right\|^{2}
$$

for any $a \in \mathfrak{N}_{h}$. Remembering that the range of $\eta$ is dense in $\mathcal{H}$ we see that there exists a unique isometry $U: \mathcal{H} \longrightarrow \widetilde{\mathcal{H}}$ such that

$$
U \eta(\pi(a))=\sum_{i \in I}^{\oplus} \eta_{i}\left(\pi_{i}(a)\right)
$$

Therefore $\mathcal{H}$ is isomorphic to a subspace of a separable Hilbert space. It shows that $\mathcal{H}$ is separable.

Let $i \in I$. Then $h_{i}\left(a^{*} a\right) \leq h\left(a^{*} a\right)$ for any $a \in \mathfrak{N}_{h}$. Combining this relation with the Schwartz inequality one can easily verify that $\left|h_{i}(a)\right| \leq \sqrt{\left\|h_{i}\right\|}\|\eta(\pi(a))\|$. Therefore there exists a vector $\Omega_{i} \in \mathcal{H}$ such that

$$
h_{i}(a)=\left(\Omega_{i} \mid \eta(\pi(a))\right)
$$

for any $a \in \mathfrak{N}_{h}$. One can also easily show that $(\eta(\pi(c)), \eta(\pi(b))) \longrightarrow h_{i}\left(c^{*} b\right)$ is a continuous sesquilinear form on the range of $\eta$. Therefore there exists a bounded positive operator $T_{i}$ acting on $\mathcal{H}$ such that

$$
h_{i}\left(c^{*} b\right)=\left(\eta(\pi(c))\left|T_{i}\right| \eta(\pi(b))\right)
$$

for any $c, b \in \mathfrak{N}_{h}$.

We shall prove that for any $x \in \mathcal{H}$ and $b \in B=\pi(A)$ we have the following equivalence:

$$
\left(\begin{array}{c}
b \in \mathcal{D}(\eta) \\
\text { and } \\
x=\eta(b)
\end{array}\right) \Longleftrightarrow\left(\begin{array}{c}
T_{i} x=b \Omega_{i} \\
\text { for all } i \in I
\end{array}\right) .
$$


Replacing in C.3 $a$ by $c^{*} b$ and comparing with (C.4 we obtain $T_{i} \eta(\pi(c))=\pi(c) \Omega_{i}$ for any $c \in \mathfrak{N}_{\varphi}$. It shows that

$$
T_{i} \eta(b)=b \Omega_{i}
$$

for any $b \in \mathcal{D}(\eta)$. This way we showed the ' $\Rightarrow$ '- part of (C.5).

We shall prove the converse. Setting in (C.4), $c=b$ and summing over $i \in I$ we see that

$$
\sum_{i \in I} T_{i}=1
$$

Assume now that $x \in \mathcal{H}, a \in A$ and $T_{i} x=\pi(a) \Omega_{i}$ for all $i \in I$ and take a sequence $\left\{a_{n}\right\}_{n=1,2, \ldots}$ of elements of $\mathfrak{N}_{h}$ converging to $a$ in norm. Using formula C.4 one can easily show that the sequence $\left\{T_{i}^{\frac{1}{2}} \eta\left(\pi\left(a_{n}\right)\right)\right\}_{n=1,2, \ldots}$ is convergent:

$$
\lim _{n \rightarrow \infty} T_{i}^{\frac{1}{2}} \eta\left(\pi\left(a_{n}\right)\right)=y_{i}
$$

where $y_{i} \in \mathcal{H}$ and $\left\|y_{i}\right\|^{2}=h_{i}\left(a^{*} a\right)$. Moreover, by formula (C.6),

$$
T_{i}^{\frac{1}{2}} y_{i}=\lim _{n \rightarrow \infty} T_{i} \eta\left(\pi\left(a_{n}\right)\right)=\lim _{n \rightarrow \infty} \pi\left(a_{n}\right) \Omega_{i}=\pi(a) \Omega_{i}=T_{i} x
$$

It shows that $y_{i}-T_{i}^{\frac{1}{2}} x \in \operatorname{ker} T_{i}^{\frac{1}{2}}$. On the other hand $y_{i}$ and $T_{i}^{\frac{1}{2}} x$ clearly belong to the closure of the range of $T_{i}^{\frac{1}{2}}$. Therefore $y_{i}-T_{i}^{\frac{1}{2}} x=0$ and $y_{i}=T_{i}^{\frac{1}{2}} x$. Now we have:

$$
h_{i}\left(a^{*} a\right)=\left\|y_{i}\right\|^{2}=\left(x\left|T_{i}\right| x\right) .
$$

Summing over $i \in I$ and using (C.7) we obtain

$$
h\left(a^{*} a\right)=\sum_{i \in I} h_{i}\left(a^{*} a\right)=(x \mid x)<\infty .
$$

It shows that $\pi(a) \in \mathcal{D}(\eta)$. Now we may use formula (C.6). It shows that $T_{i}(\eta(\pi(a))-x)=0$ and taking into account C.7 we conclude: $x=\eta(\pi(a))$. The ' $\Leftarrow$ ' of C.5 is shown.

The closedness of $\eta$ is an easy consequence of (C.5). Let $\left\{b_{\lambda}\right\}_{\lambda \in \Lambda}$ be a net of elements of $\mathcal{D}(\eta)$ converging strongly to an element $b_{\infty} \in B$, such that $x_{\lambda}=\eta\left(b_{\lambda}\right)$ converges to a vector $x_{\infty} \in \mathcal{H}$. Then $T_{i} x_{\lambda}=b_{\lambda} \Omega_{i}$. Passing to the limit we get $T_{i} x_{\infty}=b_{\infty} \Omega_{i}$. It means that $b_{\infty} \in \mathcal{D}(\eta)$ and $\eta\left(b_{\infty}\right)=x_{\infty}$.

Theorem C.3. Let $B$ a $C^{*}$-algebra acting on a separable Hilbert space $\mathcal{H}$ and $\eta: B \rightarrow \mathcal{H}$ be a closed densely defined GNS map. Then the formula:

$$
h\left(b^{*} b\right)=\left\{\begin{array}{cc}
\left(\eta^{\prime \prime}(b) \mid \eta^{\prime \prime}(b)\right) & \text { if } b \in \mathcal{D}\left(\eta^{\prime \prime}\right) \\
+\infty & \text { otherwise }
\end{array}\right.
$$

where $b$ runs over $B^{\prime \prime}$, defines a normal semifinite, weight $h$ on $B^{\prime \prime}$. Its restriction to $B$ is a locally finite lower semicontinuous weight on $B$.

Proof. Let $M=B^{\prime \prime}$. We shall use Theorem B.7 Let (B.9) be an exact vector presentation of $\eta$. Combining (B.10) with (C.8) we obtain:

$$
h\left(b^{*} b\right)=\sum_{n}\left(\Omega_{n} \mid b^{*} b \Omega_{n}\right)
$$

for all $b \in \mathcal{D}\left(\eta^{\prime \prime}\right)$. Due to the exactness, this formula holds for all $b \in M$. Therefore for any $b \in M_{+}$ we have:

$$
h(b)=\sum_{n}\left(\Omega_{n} \mid b \Omega_{n}\right) .
$$

It shows that $h$ is a normal weight on $M$ (cf. 9]). Clearly $h\left(b^{*} b\right)<\infty$ for $b \in \mathcal{D}(\eta)$. The latter set is weakly dense in $M$. Hence $h$ is semifinite. The last assertion is obvious.

Definition C.4. A weight $h$ on a $C^{*}$-algebra $A$ is said to be

(1) faithful if for any $a \in A$, the relation $h\left(a^{*} a\right)=0$ implies $a=0$. 
(2) strictly faithful if for any sequence $\left\{a_{n}\right\}_{n \in \mathbb{N}}$ in $A$ such that the sequence $\left\{h\left(a_{n}^{*} a_{n}\right)\right\}_{n \in \mathbb{N}}$ is bounded and $\lim _{n \rightarrow \infty} h\left(a_{n} a_{n}^{*}\right)=0, b=0$ is the only element of $A$ satisfying the relation

$$
h\left(\left(b-a_{n}\right)^{*}\left(b-a_{n}\right)\right) \leq h\left(a_{n}^{*} a_{n}\right)
$$

for all $n \in \mathbb{N}$.

Assume that $h$ is strictly faithful. Inserting $a_{n}=0$ in (C.9) we see that $b=0$ is the only element of $A$ such that $h\left(b^{*} b\right)=0$. It shows that any strictly faithful weight is faithful.

Lemma C.5. Let $h$ be a locally finite lower semicontinuous weight on a $C^{*}$-algebra $A$ and $(\mathcal{H}, \pi, \eta)$ be the GNS triple related to h. Then the following conditions are equivalent:

(1) The weight $h$ is strictly faithful.

(2) The weight $h$ is faithful and the mapping $S_{0}: \eta(a) \mapsto \eta\left(a^{*}\right)$ (where a runs over $\mathcal{D}(\eta) \cap \mathcal{D}(\eta)^{*}$ ) is a closable conjugate linear operator in $\mathcal{H}$.

(3) The double commutant $\eta^{\prime \prime}$ is injective.

Proof. Assume that the mapping $\eta(a) \mapsto \eta\left(a^{*}\right)$ is not closable. It is the same as to assume that the mapping $\eta\left(a^{*}\right) \mapsto \eta(a)$ is not closable. Therefore there exists a sequence $\left\{a_{n}\right\}_{n \in \mathbb{N}}$ in $\mathcal{D}(\eta) \cap \mathcal{D}(\eta)^{*}$ such that $\left\{\eta\left(a_{n}^{*}\right)\right\}_{n \in \mathbb{N}}$ in the Hilbert space $\mathcal{H}$ converges to zero in norm topology (i.e. the sequence $\left\{h\left(a_{n} a_{n}^{*}\right)\right\}_{n \in \mathbb{N}}$ of non-negative numbers converges to zero) and $\left\{\eta\left(a_{n}\right)\right\}_{n \in \mathbb{N}}$ in $\mathcal{H}$ converges to some non-zero element $x \in \mathcal{H}$ (in particular the sequence $\left\{h\left(a_{n}^{*} a_{n}\right)\right\}_{n \in \mathbb{N}}$ of nonnegative numbers is bounded). Remembering that the image of the GNS map $\eta$ is dense in $\mathcal{H}$ we find $b \in \mathcal{D}(\eta)$ such that $\|\eta(b)-x\|<\|x\|$. Clearly $b \neq 0$. By definition $x=\lim \eta\left(a_{n}\right)$. Therefore $\left\|\eta(b)-\eta\left(a_{n}\right)\right\|<\left\|\eta\left(a_{n}\right)\right\|$ for almost all $n$. This equation is equivalent to (C.9) and shows that the weight $h$ is not strictly faithful. In this way we showed that Assertion 1 implies Assertion 2.

We shall prove the converse. We now assume that the mapping $\eta(a) \mapsto \eta\left(a^{*}\right)$ is closable. Take a sequence $\left\{a_{n}\right\}_{n \in \mathbb{N}}$ in $\mathcal{D}(\eta) \cap \mathcal{D}(\eta)^{*}$ such that the sequence $\left\{\eta\left(a_{n}^{*}\right)\right\}_{n \in \mathbb{N}}$ is norm convergent to zero and the sequence $\left\{\eta\left(a_{n}\right)\right\}_{n \in \mathbb{N}}$ is bounded. Passing to a suitable subsequence, we may assume that the sequence $\left\{\eta\left(a_{n}^{*}\right)\right\}_{n \in \mathbb{N}}$ is norm convergent to zero and the sequence $\left\{\eta\left(a_{n}\right)\right\}_{n \in \mathbb{N}}$ is weakly convergent to some element $x \in \mathcal{H}$. Then the pair $(0, x)$ belongs to the (norm $\times$ weak)-closure of the Graph $S_{0}=\left\{\left(\eta\left(a^{*}\right), \eta(a)\right): a \in \mathcal{D}(\eta) \cap \mathcal{D}(\eta)^{*}\right\}$. In Hilbert spaces the weak and the norm closures of any convex set coincide. Therefore denoting by $S$ the closure of $S_{0}$ we obtain $x=S 0=0$. Now we compute as follows. Let $b$ be an element in the $C^{*}$-algebra $A$ such that the inequality (C.9) holds. Then $h\left(b^{*} b\right) \leq 2 \operatorname{Re}\left(\eta(b) \mid \eta\left(a_{n}\right)\right)$ and taking the limit $n \rightarrow \infty$, we obtain $h\left(b^{*} b\right) \leq 0$. Now the faithfulness of $h$ shows that $b=0$. This proves that Assertion 2 implies Assertion 1.

The equivalence of Assertions 2 and 3 is the well known part of the Tomita-Takesaki theory.

\section{Tomita-Takesaki theory.}

In this section we collect the main results on Tomita-Takesaki theory used in this paper. The less known results will be proven. Throughout the Section, $M$ will be a von Neumann algebra of operators acting on a Hilbert space $\mathcal{H}$. We shall assume that the embedding $M \subset \mathcal{L}(\mathcal{H})$ is standard. Then any normal semifinite faithful weight $h$ on $M$ can be represented by a closed GNS map $\eta$ densely defined on $M$ with range dense in $\mathcal{H}$, related to $h$ by the formula

$$
h\left(a^{*} a\right)=\left\{\begin{array}{cc}
(\eta(a) \mid \eta(a)) & \text { if } a \in \mathcal{D}(\eta) \\
+\infty & \text { otherwise }
\end{array}\right.
$$

for any $a \in M$. Similarly any normal semifinite faithful weight $k$ on $M^{\prime}$ can be represented by a closed GNS map $\theta^{\prime}$ densely defined on $M^{\prime}$ with range dense in $\mathcal{H}$. In what follows, $\theta$ will denote the commutant of $\theta^{\prime}$. With this notation we have:

\section{Theorem D.1.}

1. There exists unique closed conjugate linear operator $S_{\text {rel }}$ such that $\left\{\theta(a): a \in \mathcal{D}(\theta) \cap \mathcal{D}(\eta)^{*}\right\}$ is a core for $S_{\text {rel }}$ and $S_{\text {rel }} \theta(a)=\eta\left(a^{*}\right)$ for any $a \in \mathcal{D}(\theta) \cap \mathcal{D}(\eta)^{*}$.

2. The adjoint operator $F_{\text {rel }}=S_{\text {rel }}^{*}$ has a core $\left\{\theta^{\prime}(b): b \in \mathcal{D}\left(\theta^{\prime}\right) \cap \mathcal{D}\left(\eta^{\prime}\right)^{*}\right\}$ and $F_{\text {rel }} \theta^{\prime}(b)=\eta^{\prime}\left(b^{*}\right)$ for any $b \in \mathcal{D}\left(\theta^{\prime}\right) \cap \mathcal{D}\left(\eta^{\prime}\right)^{*}$.

3. The operator $\Delta_{\text {rel }}=F_{\text {rel }} S_{\text {rel }}$ depends only on the weights $h$ and $k$ (it is independent of the choice of the GNS maps $\eta$ and $\theta^{\prime}$ representing $h$ and $k$ ). 
4. The operator $J_{\mathrm{rel}}$ appearing in the polar decomposition $S_{\mathrm{rel}}=J_{\mathrm{rel}} \Delta_{\mathrm{rel}}^{1 / 2}$ is antiunitary and $J_{\mathrm{rel}} M J_{\mathrm{rel}}^{*}=M^{\prime}$.

To see that the domain of $S_{\text {rel }}$ is dense in $\mathcal{H}$ we notice that $a^{*} b \in \mathcal{D}(\theta)$ and $\left(a^{*} b\right)^{*}=b^{*} a \in \mathcal{D}(\eta)$ for any $a \in \mathcal{D}(\eta)$ and $b \in \mathcal{D}(\theta)$. Therefore $a^{*} \theta(b)=\theta\left(a^{*} b\right)$ belongs to the domain of $S_{\text {rel }}$ and $S_{\text {rel }} a^{*} \theta(b)=\eta\left(b^{*} a\right)=b^{*} \eta(a)$. It turns out that $\left\{a^{*} \theta(b): a \in \mathcal{D}(\eta), b \in \mathcal{D}(\theta)\right\}$ is a core for $S_{\text {rel. In }}$ fact one can prove a little more:

Proposition D.2. Let $\mathcal{D}_{1} \subset \mathcal{D}(\eta)$ be a core for $\eta$ and $\mathcal{D}_{2} \subset \mathcal{D}(\theta)$ be a core for $\theta$. Then

$$
\left\{a^{*} \theta(b): a \in \mathcal{D}_{1}, b \in \mathcal{D}_{2}\right\}
$$

is a core for $S_{\mathrm{rel}}$.

The similar statement holds for $F_{\text {rel }}$.

According to 3 , the relative modular operator $\Delta_{\text {rel }}$ is called the Radon-Nikodym derivative of $h$ with respect to $k$ and denoted by $\frac{d h}{d k}$. Clearly $\Delta_{\text {rel }}=\frac{d h}{d k}$ is a positive self-adjoint operator acting on $\mathcal{H}$.

Theorem D.3. Let $h$ be a weight on $M$ and $k$ be a normal semifinite faithful weight on $M^{\prime}$. Then for any $a \in M$ and $t \in \mathbb{R}$ the element

$$
\left(\frac{d h}{d k}\right)^{i t} a\left(\frac{d h}{d k}\right)^{-i t}
$$

belongs to $M$ and is independent of the choice of the weight $k$.

We set

$$
\sigma_{t}^{h}(a)=\left(\frac{d h}{d k}\right)^{i t} a\left(\frac{d h}{d k}\right)^{-i t} .
$$

Clearly $\left\{\sigma_{t}^{h}\right\}_{t \in \mathbb{R}}$ is a strongly continuous one parameter group of automorphisms of $M$. It is called the modular automorphism group related to the weight $h$.

Theorem D.4. Let $h$ and $h_{1}$ be normal semifinite faithful weights on $M$ and $k$ be a normal semifinite faithful weight on $M^{\prime}$. Then for any $t \in \mathbb{R}$ the element

$$
\left(\frac{d h}{d k}\right)^{i t}\left(\frac{d h_{1}}{d k}\right)^{-i t}
$$

belongs to $M$ and is independent of the choice of the weight $k$.

We set

$$
\left(D h: D h_{1}\right)_{t}=\left(\frac{d h}{d k}\right)^{i t}\left(\frac{d h_{1}}{d k}\right)^{-i t} .
$$

Clearly $\left(\left(D h: D h_{1}\right)_{t}\right)_{t \in \mathbb{R}}$ is a strongly continuous one parameter family of unitaries in $M$. It is called the Radon-Nikodym cocycle. One can easily verify that

$$
\begin{aligned}
\left(D h: D h_{1}\right)_{t+\tau} & =\sigma_{\tau}^{h}\left(\left(D h: D h_{1}\right)_{t}\right)\left(D h: D h_{1}\right)_{\tau} \\
& =\left(D h: D h_{1}\right)_{t} \sigma_{t}^{h_{1}}\left(\left(D h: D h_{1}\right)_{\tau}\right), \\
\sigma_{t}^{h}(a) & =\left(D h: D h_{1}\right)_{t} \sigma_{t}^{h_{1}}(a)\left(D h: D h_{1}\right)_{t}^{*}
\end{aligned}
$$

for any $t, \tau \in \mathbb{R}$ and $a \in M$.

Theorem D.5. Let $\eta$ and $\theta$ be closed GNS mappings densely defined on $M$ with the ranges dense in $\mathcal{H}, S_{\text {rel }}$ be the corresponding closed conjugate linear operator introduced in Statement 1 of Theorem D.1 and $a \in M$. Assume that $a \in \mathcal{D}(\theta)$ and $\theta(a) \in \mathcal{D}\left(S_{\text {rel }}\right)$. Then $a^{*} \in \mathcal{D}(\eta)$ and (obviously) $S_{\mathrm{rel}} \theta(a)=\eta\left(a^{*}\right)$.

Proof. By the Theorem $\mathbb{B} .2 \eta^{\prime \prime}=\eta$. Therefore it is sufficient to show that

$$
a^{*} \eta^{\prime}(b)=b S_{\mathrm{rel}} \theta(a)
$$

for any $b \in \mathcal{D}\left(\eta^{\prime}\right)$. For any $c \in \mathcal{D}\left(\theta^{\prime}\right)$ we have

$$
\begin{aligned}
\left(\theta^{\prime}(c) \mid b S_{\mathrm{rel}} \theta(a)\right) & =\left(b^{*} \theta^{\prime}(c) \mid S_{\mathrm{rel}} \theta(a)\right) \\
& =\left(\theta^{\prime}\left(b^{*} c\right) \mid S_{\mathrm{rel}} \theta(a)\right) .
\end{aligned}
$$


The reader should notice that $b^{*} c \in \mathcal{D}\left(\theta^{\prime}\right)$ and $\left(b^{*} c\right)^{*}=c^{*} b \in \mathcal{D}\left(\eta^{\prime}\right)$ (because $\mathcal{D}\left(\theta^{\prime}\right)$ and $\mathcal{D}\left(\eta^{\prime}\right)$ are left ideals in $\left.M^{\prime}\right)$. Therefore (cf. Statement 2 of Theorem D1) $\theta^{\prime}\left(b^{*} c\right) \in \mathcal{D}\left(F_{\text {rel }}\right)$ and $F_{\text {rel }} \theta^{\prime}\left(b^{*} c\right)=$ $\eta^{\prime}\left(c^{*} b\right)=c^{*} \eta^{\prime}(b)$. So we have

$$
\begin{aligned}
\left(\theta^{\prime}(c) \mid b S_{\mathrm{rel}} \theta(a)\right) & =\left(\theta^{\prime}\left(b^{*} c\right) \mid S_{\mathrm{rel}} \theta(a)\right) \\
& =\left(\theta(a) \mid F_{\mathrm{rel}} \theta^{\prime}\left(b^{*} c\right)\right) \\
& =\left(\theta(a) \mid c^{*} \eta^{\prime}(b)\right)=\left(c \theta(a) \mid \eta^{\prime}(b)\right) \\
& =\left(a \theta^{\prime}(c) \mid \eta^{\prime}(b)\right)=\left(\theta^{\prime}(c) \mid a^{*} \eta^{\prime}(b)\right)
\end{aligned}
$$

and (D.1) follows.

In the next theorem we set $\theta=\eta$. In this case we write $S, F, \Delta$ and $J$ instead of $S_{\text {rel }}, F_{\text {rel }}, \Delta_{\text {rel }}$ and $J_{\text {rel }}$. One can easily verify that $S$ and $F$ are involutive: $S^{-1}=S$ and $F^{-1}=F$. It implies that $J^{2}=1, J^{*}=J$ and $J \Delta J=\Delta^{-1}$. With this notation we have

Theorem D.6. Let $h$ be a weight associated with the closed densely defined GNS mapping with dense range. Then

1. $\mathcal{D}(\eta)$ is $\sigma^{h}$-invariant and

$$
\eta\left(\sigma_{t}^{h}(a)\right)=\Delta^{i t} \eta(a)
$$

for any $a \in \mathcal{D}(\eta)$ and $t \in \mathbb{R}$.

2. $\mathcal{D}\left(\eta^{\prime}\right)=J \mathcal{D}(\eta) J$ and

for any $a \in \mathcal{D}(\eta)$.

$$
\eta^{\prime}(J a J)=J \eta(a)
$$

We shall also use

Theorem D.7. Let $\eta$ be a closed GNS mapping densely defined on $M$ with range dense in $\mathcal{H}, h$ be the weight on $M$ related to $\eta$ and $\sigma_{i / 2}^{h}$ be the analytic generator of the corresponding modular automorphism group. Assume that $a \in \mathcal{D}(\eta)$ and $b \in \mathcal{D}\left(\sigma_{i / 2}^{h}\right)$. Then $a b \in \mathcal{D}(\eta)$ and

$$
\eta(a b)=J \sigma_{i / 2}^{h}(b)^{*} J \eta(a) .
$$

Proof. We know that $\sigma_{t}^{h}(b)=\Delta^{i t} b \Delta^{-i t}$. Let $c=\sigma_{i / 2}^{h}(b)$. Then $b \Delta^{1 / 2} \subset \Delta^{1 / 2} c$ and by passing to the adjoint operators

$$
c^{*} \Delta^{1 / 2} \subset \Delta^{1 / 2} b^{*}
$$

Let $\left\{u_{i}\right\}_{i \in I}$ be a net of positive elements of $\mathcal{D}(\eta)$ converging strongly to $I$. Then $u_{i} a$ and $\left(u_{i} a\right)^{*}=$ $a^{*} u_{i}$ belong to $\mathcal{D}(\eta)$. Therefore $\eta\left(a^{*} u_{i}\right)=S \eta\left(u_{i} a\right)=J \Delta^{1 / 2} \eta\left(u_{i} a\right)=\Delta^{-1 / 2} J \eta\left(u_{i} a\right) \in \mathcal{D}\left(\Delta^{1 / 2}\right)$. By (D.3) $b^{*}$ maps $\mathcal{D}\left(\Delta^{1 / 2}\right)$ into $\mathcal{D}\left(\Delta^{1 / 2}\right)$. Hence $\eta\left(b^{*} a^{*} u_{i}\right)=b^{*} \eta\left(a^{*} u_{i}\right) \in \mathcal{D}\left(\Delta^{1 / 2}\right)=\mathcal{D}(S)$. Using Theorem ${ }^{* * *}$ we see that $u_{i} a b=\left(b^{*} a^{*} u_{i}\right)^{*} \in \mathcal{D}(\eta)$. Remember that $J c^{*} J \in M^{\prime}$ commute with $u_{i} \in M$ we obtain

$$
\begin{aligned}
u_{i} J c^{*} J \eta(a) & =J c^{*} J u_{i} \eta(a) \\
& =J c^{*} J \eta\left(u_{i} a\right)=J c^{*} J S \eta\left(a^{*} u_{i}\right) \\
& =J c^{*} \Delta^{1 / 2} \eta\left(a^{*} u_{i}\right)=J \Delta^{1 / 2} b^{*} \eta\left(a^{*} u_{i}\right) \\
& =S \eta\left(b^{*} a^{*} u_{i}\right)=\eta\left(u_{i} a b\right) .
\end{aligned}
$$

We take the limit with respect to $i$. Since $\eta$ is closed, we conclude that $a b \in \mathcal{D}(\eta)$ and

$$
\eta(a b)=J c^{*} J \eta(a) .
$$

Clearly this formula coincides with (D.2).

The modular group and Connes' Radon-Nikodym cocycle are canonical constructions. If $\tau$ is a normal automorphism of a von Neumann algebra $M$ then for any faithful semifinite normal weight $h$ on $M, h \circ \tau$ is a faithful semifinite normal weight on $M$ and $\sigma_{t}^{h^{\circ} \tau}=\tau^{-1} \circ \sigma_{t}^{h} \mathrm{o} \tau$ for all $t \in \mathbb{R}$. Similarly for any faithful semifinite normal weights $h$ and $h_{1}$ on $M$ we have: $\left(D(h \circ \tau): D\left(h_{1} \circ \tau\right)\right)_{t}=$ $\tau^{-1}\left(\left(D h: D h_{1}\right)_{t}\right)$ for all $t \in \mathbb{R}$. For antiautomorphisms the formulae are slightly modified:

Proposition D.8. Let $M$ be a von Neumann algebra and $R$ be a normal antiautomorphism of $M$. Then

(1) For any faithful semifinite normal weight $h$ on $M$ and any $t \in \mathbb{R}$ we have

$$
\sigma_{t}^{h o} R=R^{-1}{ }_{o} \sigma_{-t^{o}} R
$$


(2) For any faithful semifinite normal weights $h$ and $h_{1}$ on $M$ and any $t \in \mathbb{R}$ we have:

$$
\left(D(h \circ R): D\left(h_{1} \circ R\right)\right)_{t}=R^{-1}\left(\left(D h_{1}: D h\right)_{-t}\right) .
$$

\section{E. Tensor Product.}

In this section we discuss the tensor product of GNS-mappings. Let $A$ be a non-degenerated $C^{*}$ algebra of operators acting on a Hilbert space $\mathcal{H}$ and $\eta: A \rightarrow \mathcal{H}$ be a densely defined closed GNS mapping. Similarly let $B$ be a non-degenerated $C^{*}$-algebra of operators acting on a Hilbert space $\mathcal{K}$ and $\theta: B \rightarrow \mathcal{K}$ be a densely defined closed GNS mapping. Then $A \otimes B$ is a non-degenerated $C^{*}$-algebra of operators acting on $\mathcal{H} \otimes \mathcal{K}$. We shall consider the algebraic tensor product $\eta \otimes$ alg $\theta$. By definition $\mathcal{D}\left(\eta \otimes_{\text {alg }} \theta\right)=\mathcal{D}(\eta) \otimes_{\text {alg }} \mathcal{D}(\theta)$. Clearly

$$
(a \otimes b)(\eta \otimes \operatorname{alg} \theta)(c)=c\left(\eta^{\prime}(a) \otimes \theta^{\prime}(b)\right)
$$

for any $c \in \mathcal{D}(\eta) \otimes_{\text {alg }} \mathcal{D}(\theta), a \in \mathcal{D}\left(\eta^{\prime}\right)$ and $b \in \mathcal{D}\left(\theta^{\prime}\right)$. Using this formula one can easily show that the mapping $\eta \otimes_{\text {alg }} \theta$ is closable. Indeed if $\left(c_{\alpha}\right)$ is a net of elements of $\mathcal{D}(\eta) \otimes_{\text {alg }} \mathcal{D}(\theta)$ converging strongly to 0 such that $\left(\eta \otimes_{\text {alg }} \theta\right)\left(c_{\alpha}\right) \rightarrow x \in \mathcal{H} \otimes \mathcal{K}$ then using (E) and passing to the limit we obtain $(a \otimes b) x=0$ for all $a \in \mathcal{D}\left(\eta^{\prime}\right)$ and $b \in \mathcal{D}\left(\theta^{\prime}\right)$. Hence $x=0$ and $\eta \otimes_{\text {alg }} \theta$ is closable.

By definition $\eta \otimes \theta$ is the closure of $\eta \otimes$ alg $\theta$. We shall prove that $\eta \otimes \theta$ is a GNS mapping acting from $A \otimes B$ into $\mathcal{H} \otimes \mathcal{K}$.

Let $a \in A, b \in B$ and $c \in \mathcal{D}(\eta \otimes \theta)$. Then there exists a net $\left(c_{\alpha}\right)$ of elements of $\mathcal{D}(\eta) \otimes_{\text {alg }} \mathcal{D}(\theta)$ such that $c_{\alpha} \rightarrow c$ strongly and $\left(\eta \otimes_{\text {alg }} \theta\right)\left(c_{\alpha}\right) \rightarrow(\eta \otimes \theta)(c)$ in norm. Passing to the limit in the obvious equality

$$
(\eta \otimes \text { alg } \theta)\left((a \otimes b) c_{\alpha}\right)=(a \otimes b)\left(\eta \otimes_{\text {alg }} \theta\right)\left(c_{\alpha}\right)
$$

we conclude that $(a \otimes b) c \in \mathcal{D}(\eta \otimes \theta)$ and

$$
(\eta \otimes \operatorname{alg} \theta)((a \otimes b) c)=(a \otimes b)(\eta \otimes \operatorname{alg} \theta)(c) .
$$

Using the same method one may replace in the above statement $a \otimes b$ by any element of $A \otimes B$. It shows that $\eta \otimes \theta$ is a GNS-mapping.

Remark E.1. By our definition $\mathcal{D}(\eta) \otimes_{\text {alg }} \mathcal{D}(\theta)$ is a core for $\eta \otimes \theta$.

In the same way one introduces the tensor product of GNS mappings defined on von Neumann algebras.

Theorem E.2. Let $\eta: A \rightarrow \mathcal{H}$ and $\theta: B \rightarrow \mathcal{K}$ be closed densely defined GNS mappings. Then

$$
(\eta \otimes \theta)^{\prime}=\eta^{\prime} \otimes \theta^{\prime}
$$

Proof. For simplicity assume that $\eta^{\prime \prime}$ and $\theta^{\prime \prime}$ are faithful and have dense ranges. Then $(\eta \otimes \theta)^{\prime \prime}$ is also faithful and has dense range. We may use Tomita-Takesaki theory. Using Proposition D.2 with $\theta=\eta$ replaced by $\eta \otimes \theta$ and $D_{1}=D_{2}$ replaced by $\mathcal{D}(\eta) \otimes$ alg $\mathcal{D}(\theta)$ one can easily show that the operator $S$ related to $(\eta \otimes \theta)^{\prime \prime}$ is the tensor product of operators $S$ related to $\eta$ and $\theta$. Consequently we have the same statements for operators $\Delta$ and $J$. Now (E.1) follows from Statement 2 of Theorem D.6.

Corollary E.3. Let

$$
\eta(a)=\sum_{\ell} a \Omega_{\ell} \quad \text { and } \quad \theta(b)=\sum_{k} b \Psi_{k}
$$

be exact vector presentation of GNS mappings $\eta$ and $\theta$. Then

$$
(\eta \otimes \theta)(c)=\sum_{k, \ell} c\left(\Omega_{\ell} \otimes \Omega_{k}\right)
$$

is an exact vector presentation of $\eta \otimes \theta$.

Proof. Let $P_{\ell} \in \mathcal{L}(\mathcal{H})$ be the orthogonal projection onto $\overline{A \Omega_{\ell}}$ and $Q_{k} \in \mathcal{L}(\mathcal{K})$ be the orthogonal projection onto $\overline{B \Psi_{k}}$. Then $P_{\ell} \otimes Q_{k}$ is the orthogonal projection onto $\overline{(A \otimes B)\left(\Omega_{\ell} \otimes \Psi_{k}\right)}$ According to Proposition $\mathrm{B} .8$ the left ideal in $A^{\prime}$ (in $B^{\prime}$ resp.) generated by $\left\{P_{1}, P_{2}, \ldots\right\}$ (by $\left\{Q_{1}, Q_{2}, \ldots\right\}$ resp.) is a core for $\eta^{\prime}$ (for $\theta^{\prime}$ resp.). Therefore the left ideal generated by $\left\{P_{\ell} \otimes Q_{k}: \ell, k=1,2, \ldots\right\}$ is a core of $\eta^{\prime} \otimes \theta^{\prime}=(\eta \otimes \theta)^{\prime}$. Using again Proposition B.8 we see that (E.2) is an exact vector presentation. 
F. Analytic generators. In this Section we give necessary information on analytic generators of one parameter groups of isometries of Banach spaces. Let $B$ be a Banach space and $\left\{\alpha_{t}\right\}_{t \in \mathbb{R}}$ be a one parameter group of isometries of $B:\left\|\alpha_{t}(a)\right\|=\|a\|, \alpha_{t}\left(\alpha_{s}(a)\right)=\alpha_{t+s}(a)$ and $\alpha_{0}(a)=a$ for any $a \in B$ and $t, s \in \mathbb{R}$. We shall always assume that the group is pointwise continuous: for any $a \in B,\left\|\alpha_{t}(a)-a\right\| \rightarrow 0$ when $t \rightarrow 0$.

Let us recall [40] that the analytical generator $\alpha_{i}$ of the group $\left\{\alpha_{t}\right\}_{t \in \mathbb{R}}$ is the linear operator acting on $B$ in the following way:

For any $a, b \in B: a \in \mathcal{D}\left(\alpha_{i}\right)$ and $b=\alpha_{i}(a)$ if and only if there exists a mapping $z \mapsto a_{z} \in B$ continuous on the strip $\{z \in \mathbb{C}: \Im z \in[0,1]\}$ and holomorphic in the interior of this strip such that $a_{t}=\alpha_{t}(a)$ for all $t \in \mathbb{R}$ and $a_{i}=b$.

It is known that $\alpha_{i}$ is a closed densely defined linear mapping.

Proposition F.1. Let $B_{k}$ be Banach spaces, $\left\{\alpha_{t}^{k}\right\}_{t \in \mathbb{R}}$ be pointwise continuous one parameter groups of isometries of $B_{k}$ and $\alpha_{i}^{k}$ be the corresponding analytical generators $(k=0,1, \ldots, K-1)$.

Moreover let

$$
\Psi: B_{0} \times B_{1} \times \cdots \times B_{K-1} \longrightarrow \mathbb{C}
$$

be a continuous K-linear form such that

$$
\Psi\left(\alpha_{i}^{0}\left(a_{0}\right), \alpha_{i}^{1}\left(a_{1}\right), \ldots, \alpha_{i}^{K-1}\left(a_{K-1}\right)\right)=\Psi\left(a_{0}, a_{1}, \ldots, a_{K-1}\right)
$$

for any $a_{k} \in \mathcal{D}\left(\alpha_{i}^{k}\right)(k=0,1, \ldots, K-1)$. Then

$$
\Psi\left(\alpha_{t}^{0}\left(a_{0}\right), \alpha_{t}^{1}\left(a_{1}\right), \ldots, \alpha_{t}^{K-1}\left(a_{K-1}\right)\right)=\Psi\left(a_{0}, a_{1}, \ldots, a_{K-1}\right)
$$

for any $t \in \mathbb{R}$ and $a_{k} \in B_{k}(k=0,1, \ldots, K-1)$.

To prove this proposition we shall use the following result known as Carlson's Lemma (cf. 28, page 228]):

Proposition F.2. Let $f(\cdot)$ be a holomorphic function on the half-plane $\mathbb{C}_{+}=\{z \in \mathbb{C}: \Im z>0\}$. Assume that for some constant $M \in \mathbb{R}, f(z) e^{-M \Im z}$ is bounded on $\mathbb{C}_{+}$and that $f($ in $)=0$ for $n \in \mathbb{N}$. Then $f$ vanishes identically.

Proof of Proposition $\mathbf{F . 1}$ Let $\overline{\mathbb{C}}_{+}=\mathbb{C}_{+} \cup \mathbb{R}$ be the closure of $\mathbb{C}_{+}$. For any $k=1,2, \ldots, K$ we set

$$
\mathcal{D}_{k}=\left\{\begin{array}{cc}
\text { there exists continuous map: } & \overline{\mathbb{C}}_{+} \ni z \longrightarrow a(z) \in B_{k} \\
a \in B_{k}: & \text { holomorphic on } \mathbb{C}_{+} \text {such that for some constant } M \in \mathbb{R}, \\
a(z) e^{-M \Im z} \text { is bounded on } \overline{\mathbb{C}}_{+} \text {and } a(t)=\alpha_{t}^{k}(a) \text { for all } t \in \mathbb{R}
\end{array}\right\} .
$$

Let $a_{k} \in \mathcal{D}_{k}(k=0,1, \ldots, K-1), a_{k}(z)$ be the holomorphic extension of $\alpha_{t}^{k}\left(a_{k}\right)$ (cf. the above definition of $\mathcal{D}_{k}$ ) and

$$
g(z)=\Psi\left(a_{0}(z), a_{1}(z), \ldots, a_{K-1}(z)\right) .
$$

Then, for any $n \in \mathbb{N}, a_{k} \in \mathcal{D}\left(\left(\alpha_{i}^{k}\right)^{n}\right)$ and $\left(\alpha_{i}^{k}\right)^{n} a_{k}=a_{k}(n i)$. Taking into account Assumption (F.1) we see that $g(i n)=g(0)$. Now one can easily show that the function $f(z)=g(z)-g(0)$ satisfies the assumptions of the Carlson's lemma. Therefore $g(z)=g(0)$ for all $z \in \overline{\mathbb{C}}_{+}$. For real $z$ this formula coincides with $(\mathrm{F} .2$.

To end the proof we have to show that for each $k, \mathcal{D}_{k}$ is dense in $B_{k}$. Let $a \in B_{k}$. For any $\varepsilon>0$ we set

$$
a_{\varepsilon}=\frac{1}{2 \pi} \int_{\mathbb{R}} \frac{e^{1+i \tau}}{(1+i \tau)^{2}} \alpha_{\varepsilon \tau}^{k}(a) d \tau
$$

It turns out that $a_{\varepsilon} \in \mathcal{D}_{k}$. Indeed one can easily verify that the map

$$
\overline{\mathbb{C}}_{+} \ni z \longrightarrow a_{\varepsilon}(z)=\frac{e^{-i \frac{z}{\varepsilon}}}{2 \pi} \int_{\mathbb{R}} \frac{e^{1+i \tau}}{\left(1-i \frac{z}{\varepsilon}+i \tau\right)^{2}} \alpha_{\varepsilon \tau}^{k}(a) d \tau \in B_{k}
$$

is the holomorphic continuation of $\alpha_{t}^{k}\left(a_{\varepsilon}\right)$ satisfying all the requirements of definition of $\mathcal{D}_{k}$. Using the Lebesgue dominating convergence theorem, one can easily show that

$$
\lim _{\varepsilon \rightarrow 0} a_{\varepsilon}=\left(\frac{1}{2 \pi} \int_{\mathbb{R}} \frac{e^{1+i \tau}}{(1+i \tau)^{2}} d \tau\right) a=a .
$$

It shows that any element of $B_{k}$ is a norm-limit of elements of $\mathcal{D}_{k}$. 
Theorem F.3. Let $B_{k}$ be Banach spaces, $\left\{\alpha_{t}^{k}\right\}_{t \in \mathbb{R}}$ be pointwise continuous one parameter groups of isometries of $B_{k}$ and $\alpha_{i}^{k}$ be the corresponding analytical generators $(k=1,2, \ldots, K)$.

Moreover let

$$
\Psi: B_{1} \times B_{2} \times \cdots \times B_{K-1} \longrightarrow B_{K}
$$

be a continuous $(K-1)$-linear mapping such that

and

$$
\Psi\left(a_{1}, a_{2}, \ldots, a_{K-1}\right) \in \mathcal{D}\left(\alpha_{i}^{K}\right)
$$

$$
\alpha_{i}^{K}\left(\Psi\left(a_{1}, a_{2}, \ldots, a_{K-1}\right)\right)=\Psi\left(\alpha_{i}^{1}\left(a_{1}\right), \alpha_{i}^{2}\left(a_{2}\right), \ldots, \alpha_{i}^{K-1}\left(a_{K-1}\right)\right)
$$

for any $a_{k} \in \mathcal{D}\left(\alpha_{i}^{k}\right)(k=1,2, \ldots, K-1)$. Then

$$
\alpha_{t}^{K}\left(\Psi\left(a_{1}, a_{2}, \ldots, a_{K-1}\right)\right)=\Psi\left(\alpha_{t}^{1}\left(a_{1}\right), \alpha_{t}^{2}\left(a_{2}\right), \ldots, \alpha_{t}^{K-1}\left(a_{K-1}\right)\right)
$$

for any $t \in \mathbb{R}$ and $a_{k} \in B_{k}(k=1,2, \ldots, K-1)$.

Proof. Let $B_{0}$ be the set of all $a \in B_{K}^{*}$ such that the mapping $t \in \mathbb{R} \rightarrow a \circ \alpha_{t}^{K} \in B_{K}^{*}$ is norm continuous. Then $B_{0}$ is a closed subspace of $B_{K}^{*}$. For each $a \in B_{K}^{*}$ we set

$$
\mathcal{R}_{\varepsilon}(a)=\frac{1}{\sqrt{\varepsilon \pi}} \int_{\mathbb{R}} a \circ \alpha_{t}^{K} e^{-t^{2} / \varepsilon} d t
$$

Then

$$
\mathcal{R}_{\varepsilon}(a) \circ \alpha_{s}^{K}-\mathcal{R}_{\varepsilon}(a)=\frac{1}{\sqrt{\varepsilon \pi}} \int_{\mathbb{R}} a \circ \alpha_{t}^{K}\left(e^{-(t-s)^{2} / \varepsilon}-e^{-t^{2} / \varepsilon}\right) d t
$$

and hence $\mathcal{R}_{\varepsilon}(a) \in B_{0}$. Since

$$
\mathcal{R}_{\varepsilon}(a)-a=\frac{1}{\sqrt{\varepsilon \pi}} \int_{\mathbb{R}}\left(a \circ \alpha_{t}^{K}-a\right) e^{-t^{2} / \varepsilon} d t
$$

$B_{0}$ is weakly* dense in $B_{K}^{*}$. The canonical bilinear form on $B_{0} \times B_{K}$, will be denoted by $\langle\cdot, \cdot\rangle$. Since $\mathcal{R}_{\varepsilon}(a) \circ \alpha_{t}^{K}=\mathcal{R}_{\varepsilon}\left(a \circ \alpha_{t}^{K}\right)$ for all $t \in \mathbb{R}$, the formula

$$
\left\langle\alpha_{t}^{0}\left(a_{0}\right), \alpha_{t}^{K}\left(a_{K}\right)\right\rangle=\left\langle a_{0}, a_{K}\right\rangle,
$$

where $a_{0} \in B_{0}, a_{K} \in B_{K}, t \in \mathbb{R}$, defines a one parameter group $\left\{\alpha_{t}^{0}\right\}_{t \in \mathbb{R}}$ of isometries of $B_{0}$. Let $\alpha_{i}^{0}$ be the corresponding analytical generator. By the holomorphic continuation we have:

$$
\left\langle\alpha_{i}^{0}\left(a_{0}\right), \alpha_{i}^{K}\left(a_{K}\right)\right\rangle=\left\langle a_{0}, a_{K}\right\rangle
$$

for any $a_{0} \in \mathcal{D}\left(\alpha_{i}^{0}\right)$ and $a_{K} \in \mathcal{D}\left(\alpha_{i}^{K}\right)$.

For any $a_{k} \in \mathcal{D}\left(\alpha_{i}^{k}\right)(k=0,1, \ldots, K-1)$ we set:

$$
\Psi^{\prime}\left(a_{0}, a_{1}, \ldots, a_{K-1}\right)=\left\langle a_{0}, \Psi\left(a_{1}, \ldots, a_{K-1}\right)\right\rangle .
$$

Clearly $\Psi^{\prime}$ is a continuous K-linear form defined on $B_{0} \times B_{1} \times \cdots \times B_{K-1}$. Using (F.8) and (F.5) one can easily check that

$$
\Psi^{\prime}\left(\alpha_{i}^{0}\left(a_{0}\right), \alpha_{i}^{1}\left(a_{1}\right), \ldots, \alpha_{i}^{K-1}\left(a_{K-1}\right)\right)=\Psi^{\prime}\left(a_{0}, a_{1}, \ldots, a_{K-1}\right)
$$

for any $a_{k} \in \mathcal{D}\left(\alpha_{i}^{k}\right)(k=0,1, \ldots, K-1)$. Now, Proposition F.1 shows that

$$
\left\langle\alpha_{t}^{0}\left(a_{0}\right), \Psi\left(\alpha_{t}^{1}\left(a_{1}\right), \ldots, \alpha_{t}^{K-1}\left(a_{K-1}\right)\right)\right\rangle=\left\langle a_{0}, \Psi\left(a_{1}, \ldots, a_{K-1}\right)\right\rangle
$$

for any $a_{k} \in B_{k}(k=0,1, \ldots, K-1)$ and $t \in \mathbb{R}$. Comparing this result with (F.7) we get (F.6).

In particular, inserting in Theorem F.3 $K=2, B_{1}=B_{2}=B$ and $\Psi=\operatorname{id}_{B}$ we get

Corollary F.4. Let B be a Banach space and $\left\{\alpha_{t}^{1}\right\}_{t \in \mathbb{R}}$ and $\left\{\alpha_{t}^{2}\right\}_{t \in \mathbb{R}}$ be one parameter groups of isometries acting on $B$. Assume that the analytic generators $\alpha_{i}^{1} \subset \alpha_{i}^{2}$. Then $\alpha_{i}^{1}=\alpha_{i}^{2}$ and $\alpha_{t}^{1}=\alpha_{t}^{2}$ for all $t \in \mathbb{R}$.

Let us go back to Theorem F.3 Assume for the moment that the image of (F.3) is linearly dense in $B_{K}$. Then the linear span of elements of the form (F.4 is a core for $\alpha_{i}^{K}$. This fact follows from the following

Theorem F.5. Let $\alpha=\left\{\alpha_{t}\right\}_{t \in \mathbb{R}}$ be a one parameter group of isometries acting on a Banach space $B$ and $\mathcal{D}$ be an $\alpha$-invariant linear subset of $\mathcal{D}\left(\alpha_{i}\right)$. Assume that $\mathcal{D}$ is dense in $B$. Then $\mathcal{D}$ is a core for $\alpha_{i}$. 
To prove this Theorem we shall use the regularizing operator $\mathcal{R}_{\alpha}$ acting on $B$. By definition

$$
\mathcal{R}_{\alpha}=\frac{1}{\sqrt{\pi}} \int_{-\infty}^{\infty} e^{-\tau^{2}} \alpha_{\tau} d \tau
$$

One can easily verify that $\mathcal{R}_{\alpha}$ is a norm one operator acting on $B$, the range $\mathcal{R}_{\alpha}(B) \subset \mathcal{D}\left(\alpha_{i}\right)$ and

$$
\alpha_{i} \circ \mathcal{R}_{\alpha}=\frac{1}{\sqrt{\pi}} \int_{-\infty}^{\infty} e^{-(\tau-i)^{2}} \alpha_{\tau} d \tau
$$

is a bounded operator acting on $B$ with norm less or equal $e$. Endowing $\mathcal{D}\left(\alpha_{i}\right)$ with the graph norm $\left(\|b\|_{\text {Graph }}=\|b\|+\left\|\alpha_{i}(b)\right\|\right.$ for any $\left.b \in \mathcal{D}\left(\alpha_{i}\right)\right)$ we see that $\mathcal{R}_{\alpha}$ maps $B$ into $\mathcal{D}\left(\alpha_{i}\right)$ in a continuous way. If $\mathcal{D}$ is a dense subset of $B$, then $\mathcal{R}_{\alpha}(\mathcal{D})$ is a dense subset of $\mathcal{R}_{\alpha}(B)$ in the sense of the graph topology of $\mathcal{D}\left(\alpha_{i}\right)$. It means that the restrictions of $\alpha_{i}$ to $\mathcal{R}_{\alpha}(\mathcal{D})$ and $\mathcal{R}_{\alpha}(B)$ have the same closures:

$$
\overline{\left.\alpha_{i}\right|_{\mathcal{R}_{\alpha}(\mathcal{D})}}=\overline{\left.\alpha_{i}\right|_{\mathcal{R}_{\alpha}(B)}} .
$$

Proposition F.6. Let $\alpha=\left\{\alpha_{t}\right\}_{t \in \mathbb{R}}$ be a one parameter group of isometries acting on a Banach space $B, b \in B$ and $\mathcal{D} \subset B$ be an $\alpha$-invariant linear subset. Then

1. $\mathcal{R}_{\alpha}(b)$ belongs to the smallest closed $\alpha$-invariant linear subspace of $B$ containing $b$.

2. $b$ belongs to the smallest closed $\alpha$-invariant linear subspace of $B$ containing $\mathcal{R}_{\alpha}(b)$.

3. $\mathcal{R}_{\alpha}(\mathcal{D})$ and $\mathcal{D}$ have the same closures:

$$
\overline{\mathcal{R}_{\alpha}(\mathcal{D})}=\overline{\mathcal{D}} .
$$

Proof. Ad 1 and 2. It is sufficient to show that for any continuous linear functional $\varphi$ on $B$ we have:

$$
\left(\begin{array}{c}
\varphi\left(\alpha_{t}(b)\right)=0 \\
\text { for all } t \in \mathbb{R}
\end{array}\right) \Longleftrightarrow\left(\begin{array}{c}
\varphi\left(\alpha_{t}\left(\mathcal{R}_{\alpha}(b)\right)\right)=0 \\
\text { for all } t \in \mathbb{R}
\end{array}\right)
$$

For any $\varphi \in B^{d}$ we set: $f_{\varphi}(t)=\varphi\left(\alpha_{t}(b)\right)$ and $g_{\varphi}(t)=\varphi\left(\alpha_{t}\left(\mathcal{R}_{\alpha}(b)\right)\right)$. Then $f_{\varphi}$ and $g_{\varphi}$ are bounded continuous functions on $\mathbb{R}$ and may be considered as tempered distributions on $\mathbb{R}$. In what follows, $\widetilde{f}_{\varphi}$ and $\widetilde{g}_{\varphi}$ will denote the Fourier transform of $f_{\varphi}$ and $g_{\varphi}$. Therefore (F.11) is equivalent to

$$
\left(\tilde{f}_{\varphi}=0\right) \Longleftrightarrow\left(\widetilde{g}_{\varphi}=0\right) \text {. }
$$

Taking into account (F.9) we obtain:

$$
\varphi\left(\mathcal{R}_{\alpha}(b)\right)=\frac{1}{\sqrt{\pi}} \int_{-\infty}^{\infty} e^{-\tau^{2}} \varphi\left(\alpha_{\tau}(b)\right) d \tau
$$

Replacing $b$ by $\alpha_{t}(b)$ we obtain

$$
g_{\varphi}(t)=\frac{1}{\sqrt{\pi}} \int_{-\infty}^{\infty} e^{-\tau^{2}} f_{\varphi}(t+\tau) d \tau=\frac{1}{\sqrt{\pi}} \int_{-\infty}^{\infty} e^{-(t-\tau)^{2}} f_{\varphi}(\tau) d \tau
$$

On the right hand side we have the convolution product of the Gaussian function $\frac{1}{\pi} e^{-\tau^{2}}$ with $f_{\varphi}$. Passing to the Fourier transform we see that $\widetilde{g}_{\varphi}$ equals to $\widetilde{f}_{\varphi}$ multiplied by the Fourier transform of the Gaussian function. The latter has no zero points in $\mathbb{R}$ and (F.12) follows.

Ad 3. It follows immediately from Statements 1 and 2.

Now we are able to prove Theorem F.5

Proof. We shall use Statement 3 of Proposition F.6 in the following context. Instead of $B$ we take $\mathcal{D}\left(\alpha_{i}\right)$ endowed with the graph norm. Clearly $\alpha$ is a group of isometries of $\mathcal{D}\left(\alpha_{i}\right)$. Therefore for any $\alpha$-invariant subset $\mathcal{D} \subset \mathcal{D}\left(\alpha_{i}\right)$, the closures (in the sense of the graph topology) of $\mathcal{R}_{\alpha}(\mathcal{D})$ and $\mathcal{D}$ coincide. In other words

$$
\overline{\left.\alpha_{i}\right|_{\mathcal{R}_{\alpha}(\mathcal{D})}}=\overline{\left.\alpha_{i}\right|_{\mathcal{D}}} .
$$

Inserting in this formula $\mathcal{D}=\mathcal{D}\left(\alpha_{i}\right)$ and using the inclusion $\mathcal{D}\left(\alpha_{i}\right) \subset B$ we obtain

$$
\overline{\left.\alpha_{i}\right|_{\mathcal{R}_{\alpha}(B)}}=\alpha_{i} .
$$

Combining now ( $(\underline{\mathrm{F} .10}),(\underline{\mathrm{F} .13})$ and $(\underline{\mathrm{F} .14})$ we get: $\overline{\left.\alpha_{i}\right|_{\mathcal{D}}}=\alpha_{i}$. It means that $\mathcal{D}$ is a core for $\alpha_{i}$. 
Theorem F.7. Let $\alpha=\left\{\alpha_{t}\right\}_{t \in \mathbb{R}}$ be a one parameter group of isometries acting on a Banach space $B$ and $a, b \in B$. Assume that $\alpha_{i}{ }^{o} \mathcal{R}_{\alpha}(a)=\mathcal{R}_{\alpha}(b)$. Then $a \in \mathcal{D}\left(\alpha_{i}\right)$ and $\alpha_{i}(a)=b$.

Proof. We shall use Statement 2 of Proposition F.6 in the following context. Instead of $B$ we take $\widetilde{B}=B \oplus B$. Clearly $\left\{\widetilde{\alpha}_{t}\right\}_{t \in \mathbb{R}}$, where $\widetilde{\alpha}_{t}=\alpha_{t} \oplus \alpha_{t}$ for all $t \in \mathbb{R}$, is a group of isometries of $\widetilde{B}$. $\operatorname{Graph}\left(\alpha_{i}\right)=\left\{\left(a, \alpha_{i}(a)\right): a \in \mathcal{D}\left(\alpha_{i}\right)\right\}$ is a closed, $\widetilde{\alpha}$-invariant subspace of $\widetilde{B}$. We assumed that $\mathcal{R}_{\tilde{\alpha}}(a, b)=\left(\mathcal{R}_{\alpha}(a), \mathcal{R}_{\alpha}(b)\right) \in \operatorname{Graph}\left(\alpha_{i}\right)$. Using Statement 2 of Proposition F.6 and remembering that $\operatorname{Graph}\left(\alpha_{i}\right)$ is $\widetilde{\alpha}$ invariant we see that $(a, b) \in \operatorname{Graph}\left(\alpha_{i}\right)$. It means that $a \in \mathcal{D}\left(\alpha_{i}\right)$ and $b=\alpha_{i}(a)$.

Remark F.8. One can easily generalize Proposition F.1 and Theorem F.3 for mappings $\Psi$ that are conjugate linear with respect to some variable (and linear with respect to remaining ones). If $\Psi\left(a_{1}, a_{2}, \ldots, a_{K}\right)$ is conjugate linear with respect to $a_{k}$, then in all entries the $\alpha_{i}^{k}$ should be replaced by $\alpha_{-i}^{k}$ (the analytic generator of $\left\{\alpha_{-t}^{k}\right\}_{t \in \mathbb{R}}$ ).

\section{REFERENCES}

[1] S. Baaj et G. Skandalis, Unitaries multiplicatifs et dualité pour les produits croisé de $C^{*}$-algèbres, Ann. Sci. Ec. Norm. Sup., $4^{e}$ série, 26(1993), 425-488.

[2] R.P. Boas, Entire Functions, Academic Press Inc., New York, 1954.

[3] A. Connes, On the spatial theory on von Neumann algebras, J. Functional Analysis 35(1980), 153-164.

[4] J. Dixmier, Les algèbres d'opérateur dans l'espace Hilbertien, Gauthier-Villars, Paris, 1957.

[5] R.S. Doran and J. Wichmann: Approximate identities and factorization in Banach modules. Lecture Notes in Math., 768(1979), Springer Verlag.

[6] V.G. Drinfeld, Quantum groups, in Proceedings of the ICM, Ed. by A. Gleason, AMS, Rhode Island (1987), $798-820$.

[7] M. Enock et J.M. Schwartz, Kac Algebras and Duality of Locally Compact Groups, Springer-Verlag, 1992.

[8] M. Enock et J.M. Vallin, $C^{*}$-algèbres de Kac et algèbres de Kac, Proc. London Math. Soc. (3)66(1993), 619-650.

[9] U. Haagerup, Normal weights on $W^{*}$-algebras, J. Functional Analysis, 19(1975), 302-317.

[10] E. Koelink and J. Kustermans, A locally compact quantum group analogue of the normalizer of $S U(1,1)$ in $S L(2, \mathbb{C})$, arXiv: math. QA/ 0105117 v1 May 2001.

[11] L. Korogodski, Quantum Group $S U(1,1) \times \mathbb{Z}_{2}$ and "Super Tensor" Products, Commun. Math. Phys., 163(1994), $433-460$.

[12] H. Kurose and Y. Nakagami, Compact Hopf *-algebras, quantum enveloping algebras and dual Woronowicz algebras for quantum Lorentz groups, International J. Math., 8(1997), 959-997.

[13] J. Kustermans, Locally compact quantum groups in the universal setting, International J. Math., 12(2001), 289-338.

[14] J. Kustermans and S. Vaes, A simple definition for locally compact quantum groups, C. R. Acad. Sci. Paris, Ser. I, (10)328(1999), 871-876.

[15] J. Kustermans and S. Vaes, Locally compact quantum groups, Ann. Sci. Ec. Norm. Sup., (4)33(2000), $837-934$.

[16] J. Kustermans and S. Vaes, The operator algebra approach to quantum groups, Proc. Nat. Acad. Sci. U. S. A., to appear.

[17] T. Masuda, On some points of the departure of the quantum groups from the classical ordinary Lie groups, Proceedings of the Workshop on Quantum Groups, Palermo, December 1997, Ed. by D. Kastler et. al. Nova Science, New York (1999).

[18] T. Masuda and Y. Nakagami, An operator algebraic framework for the duality of the quantum groups, "Mathematical Physics X', in Proc. AMP-91, Univ. Leipzig, 1991, Ed. by K. Schmüdgen, Springer-Verlag, BerlinHeiderberg-New York, (1992), 291-295.

[19] T. Masuda and Y. Nakagami, A von Neumann algebra framework for the duality of the quantum groups, Publ. RIMS Kyoto Univ., 30(1994), 799-850.

[20] Y. Nakagami, Double group construction for compact Woronowicz algebras, International J. Math., 7(1996), $521-540$

[21] G.K. Pedersen and M. Takesaki, The Radon-Nikodym theorem for von Neumann algebras, Acta Math., 130(1973), 53-87.

[22] P. Podles̀ and S.L. Woronowicz, Quantum deformation of Lorentz group, Commun. Math. Phys., 130(1990), 381-431.

[23] S. Stratilla, Modular Theory in Operator Algebras, Editura Academiei, Abacus Press, 1981.

[24] M. Takesaki, Tomita's theory of modular Hilbert algebras and its applications, Lecture Notes in Math., 128(1970), Springer-Verlag.

[25] H.F. Trotter, On the product of semigroups of operators, Proc. Amer. Math. Soc., 10(1959), 545 - 551.

[26] J.M. Vallin, $C^{*}$-algèbres de Hopf et $C^{*}$-algèbres de Kac, Proc. London Math. Soc.,(3)50(1985), 131-174.

[27] A. Van Daele: The Haar measure on some locally compact groups, Preprint of the K.U. Leuven (2000).

[28] S.L. Woronowicz, On the purification of factor states, Commun. Math. Phys., 28(1972), 221-235.

[29] S.L. Woronowicz, Pseudospaces, pseudogroups and Pontryagin duality, Proceedings of the International Conference on Mathematical Physics, Lausanne 1979. Lecture Notes in Physics, 116(1979), 407-412. Berlin, Heidelberg, New York, Springer-Verlag .

[30] S.L. Woronowicz, Unbounded elements affiliated with $C^{*}$-algebras and non-compact quantum groups, Commun. Math. Phys., 136(1991), 399-432.

[31] S.L. Woronowicz, $C^{*}$-algebras generated by unbounded elements, Reviews Math. Phys., 7(1995), 481-521. 
[32] S.L. Woronowicz, Quantum E(2)-group and its Pontryagin dual, Lett. Math. Phys., 23(1991), 251-263.

[33] S.L. Woronowicz, Compact quantum groups, Les Houches, Session LXIV, 1995, Quantum Symmetries, (1998), 845-884, Elsevier.

[34] S.L. Woronowicz, From multiplicative unitaries to quantum groups, International J. Math., 7(1996), 127-149.

[35] S.L. Woronowicz, A series of lectures on Haar weights, Argentina, 2000.

[36] S.L. Woronowicz, Quantum az $+b$ group on complex plane, International J. Math., 12(2001), 461-503.

[37] S.L. Woronowicz and S. Zakrzewski, Quantum ' $a x+b$ ' group, Reviews in Math. Phys., 14, Nos 7 \& 8 (2002), 797-828.

[38] S.L. Woronowicz and S. Zakrzewski, Quantum Lorentz group having Gauss decomposition property. Publ. RIMS, Kyoto Univ., 28(1992), 809-824.

[39] T. Yamanouchi, Double group construction in the von Neumann algebra framework for quantum groups, J. Math. Soc. Japan, 52(2000), 807-834.

[40] L. Zsido: On the characterization of the analytic generator of *-automorphism groups in Operator algebras and applications (Kingstone Ontario 1980), in Proc. Symp. Pure Math., 38(1982), 381-383, AMS, Providence R.I.

(T. Masuda) Institute of Mathematics, University of Tsukuba, 1-1, Tennodai 1 chome, Tsukuba-Shi, IBARAKI, 305-8571 JAPAN.

E-mail address, T. MASUDA: tetsuya@math.tsukuba.ac.jp

(Y. NAKAGami) Department of Mathematical and Physical Science, Faculty of Science, Japan Women's University, 8-1, Mejirodai 2 Chome, Bunkyo-Ku, Tokyo, 112-8681 Japan.

E-mail address, Y. NAKAGAMI: nakagami@fc.jwu.ac.jp

(S. L. WOrOnowicz) Department of Mathematical Methods in Physics, Faculty of Physics, UniverSity of Warsaw, Hoża 74, 00-682 WarszaWa, Poland.

E-mail address, S. L. WORONOWICZ: slworono@fuw.edu.pl 